\title{
Multi-scale brain connectivity with diffusion MRI
}

Citation for published version (APA):

Bastiani, M. (2015). Multi-scale brain connectivity with diffusion MRI. [Doctoral Thesis, Maastricht University]. Maastricht University. https://doi.org/10.26481/dis.20150320mb

Document status and date:

Published: 01/01/2015

DOI:

10.26481/dis.20150320mb

Document Version:

Publisher's PDF, also known as Version of record

\section{Please check the document version of this publication:}

- A submitted manuscript is the version of the article upon submission and before peer-review. There can be important differences between the submitted version and the official published version of record.

People interested in the research are advised to contact the author for the final version of the publication, or visit the DOI to the publisher's website.

- The final author version and the galley proof are versions of the publication after peer review.

- The final published version features the final layout of the paper including the volume, issue and page numbers.

Link to publication

\footnotetext{
General rights rights.

- You may freely distribute the URL identifying the publication in the public portal. please follow below link for the End User Agreement:

www.umlib.nl/taverne-license

Take down policy

If you believe that this document breaches copyright please contact us at:

repository@maastrichtuniversity.nl

providing details and we will investigate your claim.
}

Copyright and moral rights for the publications made accessible in the public portal are retained by the authors and/or other copyright owners and it is a condition of accessing publications that users recognise and abide by the legal requirements associated with these

- Users may download and print one copy of any publication from the public portal for the purpose of private study or research.

- You may not further distribute the material or use it for any profit-making activity or commercial gain

If the publication is distributed under the terms of Article $25 \mathrm{fa}$ of the Dutch Copyright Act, indicated by the "Taverne" license above, 


\section{Multi-scale brain connectivity with diffusion MRI}

Matteo Bastiani 
All rights reserved. No part of this publication may be reproduced, stored in an automated data system or transmitted in any form or by any means, electronic, mechanical or photocopying, recording or otherwise, without prior permission of the author.

(C) Matteo Bastiani, Maastricht, 2015

Cover Art by Alessandro Starace

Production by CPI Wöhrmann Print Services, B.V.

ISBN 978-94-6203-805-9 


\section{Multi-scale brain connectivity with diffusion MRI}

\section{DISSERTATION}

to obtain the degree of Doctor at Maastricht University, on the authority of Rector Magnificus, Prof. Dr. L.L.G. Soete, in accordance with the decision of the Board of Deans, to be defended in public on Friday 20 ${ }^{\text {th }}$ March 2015 at 10:00 hours by Matteo Bastiani 


\section{Supervisor}

Prof. Dr. Rainer Goebel

\section{Co-supervisors}

Dr. Alard Roebroeck

Prof. Dr. Nadim Jon Shah

\section{Assessment committee}

Prof. Dr. Bernadette M. Jansma (Chair)

Prof. Dr. Timothy Behrens (University of Oxford, United Kingdom)

Prof. Dr. Elia Formisano

Dr. Marc Tittgemeyer (Max Planck Institute, Cologne, Germany)

Dr. Kamil Uludag 


\section{Contents}

Chapter 1 Introduction

Chapter 2 Human cortical connectome reconstruction from 39 diffusion weighted MRI: the effect of tractography algorithm

Chapter 3 Comparative analysis of the macroscale structural connectivity in the macaque and human brain

Chapter 4 Automatic classification of human cortical layers and areas using high resolution diffusion MRI

Chapter 5 Probing white matter microstructural 191 compartments with diffusion MRI: high spatial resolution and partial volume effects

Chapter 6 Summary and future directions

Appendix Knowledge valorization 

Chapter 1

Introduction 
During any basic course about physiology, students are taught that neurons are the fundamental processing unit of the nervous system. The propagation of the signals between different neurons is based on electrical and chemical events. Ionic currents travel along the axonal membrane and trigger the release of a neurotransmitter in the synaptic cleft. This chemical compound will be picked up at the dendrites of the next neuron that will transform the chemical input into another electrical signal. Depending on how neurons are wired together, the signal will be carried to different regions of our brain or body to be processed further. The wiring diagram of a simple component determines the output which we will get in the end.

Similarly, during any basic course about electronics, students are taught that all electronic devices can be designed using some basic components: resistors, capacitors, inductors and transistors. Such components are characterized by different electrical properties and, when connected to each other, they transform the input current into a more desirable output. Different ways of connecting the very same components will yield different output results. A very simple example is provided by passive filtering of a time varying input current. Suppose we are recording a signal from the outside world, e.g. human voice. First of all, a transducer will transform air movement to an electrical current. This current will be fed to a very simple circuit which filters specific frequencies. Using only two electrical elements, a capacitor and a resistor, it is possible to block the low or the high frequency components by just rearranging the cables (Fig. 1).

This analogy between electrical engineering and neuronal physiology has been used before in the study of the nervous system. Hodgkin and Huxley (1952) modeled the active and passive electrical properties of the axon by using an equivalent circuit. They recorded the electrical signal propagation along the giant squid axon and could segregate the different transmembrane current components and relate them to sodium and potassium ions. Their work is a beautiful example 
of how classical engineering approaches and basic neurophysiology can be combined to model the neuron. As a natural extension to their proposed framework, it can be said that the whole brain is a complex electrochemical device. As such, it comprises several fundamental components (i.e. neurons and neuroglia) which are highly interconnected. Therefore, another analogy can be used: to reverse engineer any electronic device, we first of all need to identify all its subparts and how they are connected to each other. The same rationale can apply to the study of the brain. One way to increase our understanding of the brain is to identify all its different sub-components and identify their afferent and efferent projections.
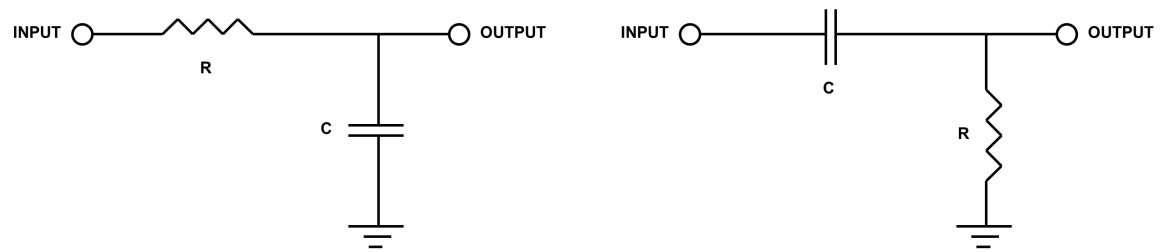

Figure 1: Schematic illustration of a low-pass (left) and high-pass (right) filter obtained combining a resistor $(\mathrm{R})$ and a capacitor $(\mathrm{C})$.

Importantly, the nature of the sub-components and projections depends on the spatial scale at which the brain is investigated. At the macroscopic level (thousands of microns) the brain is made of different cortical areas and subcortical structures which are connected to each other via short and long axonal bundles. Zooming in and looking at the mesoscopic organization of the cortical sheet (hundreds of microns), it can be observed that every cortical area shows a different layered organization of cells and neurites and that every layer has its own intracortical connectivity pattern. Finally, from the microscopic viewpoint (tens of microns to single microns), axons in white matter are not equally distributed everywhere, but have different packing densities and diameters. Given these premises, the aim of this thesis is to explore 
and unravel the structural architecture of the brain at these different scales using a combination of techniques which are based on classical neuroanatomical histology and modern magnetic resonance imaging (MRI).

\section{Macroscopic brain organization: cortical cartography and connectomics}

When looking at a human brain from the outside, the first thing that can be observed is a complex pattern of gyri and sulci. The degree of cortical folding, i.e. the gyrification pattern, correlates with brain size in mammals (for a review see: Zilles et al., 2013). Furthermore, interspecies comparative studies have highlighted similarities and differences between the human brain and that of other mammals. When compared to the Old World monkey brain, a bigger gyrification index is found in the human prefrontal cortex (Zilles et al., 1988). The tension hypothesis (Van Essen, 1997) has proposed that the complex pattern of gyrification is dictated by mechanical factors and that cortical folding influences laminar morphology. The tension exerted by long and short-ranged axonal connections is responsible for pulling together brain regions which are connected to each other. These forces might explain the different number of neurons between gyri and sulci which might as well relate to different functional properties of gyral and sulcal regions (Hilgetag and Barbas, 2005, 2006). The development of the folded cortical profile has been alternatively explained as a result of cellular development in the gray matter hypothesis (Chenn and Walsh, 2002; Lui et al., 2011). However, rather than opposing, these view are now mostly seen as complementary.

On the other hand, when looking inside the brain, an immediate gross distinction between white matter and gray matter can be made. White matter is mainly present in the inner brain volume and in the adult human brain constitutes $40 \%$ of the total matter volume in the 
central nervous system (Morell, 1984). White matter mainly consists of bundles of axons, which can be both myelinated and unmyelinated. These bundles are the long-distance cabling substrate of the brain and are responsible for carrying signals from one remote location to another (Fig. 2). On the contrary, gray matter mainly holds the nuclei of the neurons and the short-distance cabling and it is present at the external edges and in some constrained subcortical structures, such as basal ganglia and pontine nuclei (Boucsein et al., 2011). Gray matter processes the information coming from other body or brain areas and it can be subdivided into different regions based on functional or anatomical criteria.

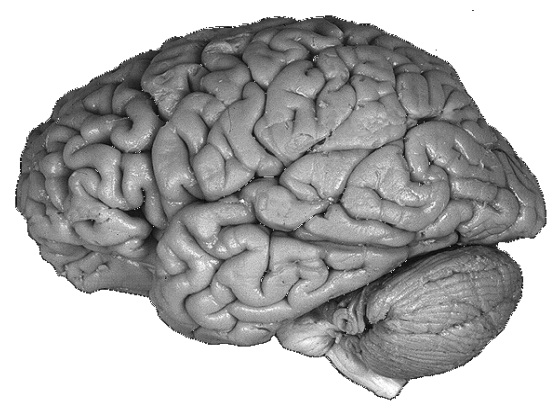

A

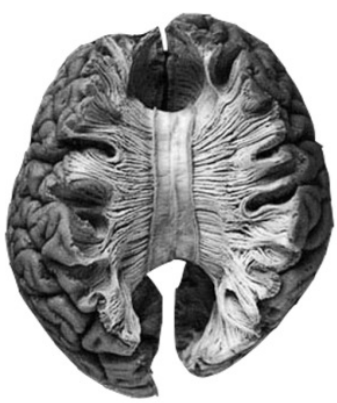

B

Figure 2: Lateral view of the left hemisphere (A) and dorsal view of the callosal fibers of a human brain, connecting the two hemispheres (B).

The first parcellations of the whole cortical mantle were published at the beginning of the $20^{\text {th }}$ century. Oskar and Cecil Vogt and their collaborator, Korbinian Brodmann, divided the brain into several cortical areas based on microanatomical features observed under the microscope. They could identify the location and the boundaries of the different brain areas using two different staining techniques. While the Vogts looked at the myelin content and its changes to delimit what they called cortical fields (Vogt, 1910), Brodmann based his parcellation on cytoarchitectural information (Brodmann, 1909), i.e. information about 
cell sizes, counts and morphology. This produced two different pictures of the organization of the brain, as Brodmann was capable of segregating 52 brain regions while the Vogts described 185 different myeloarchitectonic areas (Fig. 3). Cytoarchitectural parcellations of the cortex were brought forward by several other groups, even in very recent times (Amunts et al., 2013; Bonin and Bailey, 1947; Sanides, 1962; von Economo and Koskinas, 1925; Zilles and Amunts, 2010), while only few studies have focused on myeloarchitecture (for a review, see: Nieuwenhuys, 2013). Until today, the work done by the Vogts during the last century still represents one of the few attempts to map a comprehensive myeloarchitectonic atlas of the human brain. Recently, new methods have been introduced that can provide details about other structural features of the cortex, such as receptor mapping techniques (Amunts et al., 2010; Eickhoff et al., 2008; Geyer et al., 1998; Zilles and Amunts, 2009).

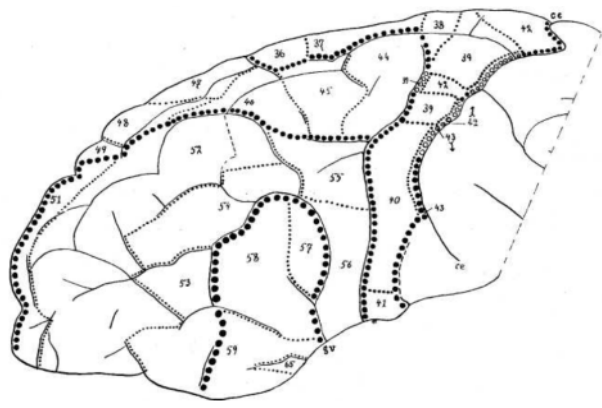

A

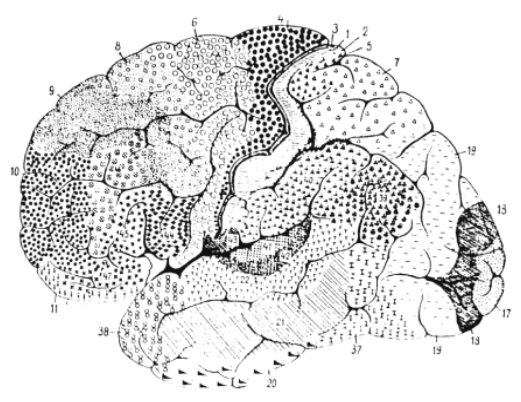

B

Figure 3: A) Vogt (1910) parcellation of the human frontal pole based on myeloarchitecture and B) Brodmann (1909) parcellation of the human brain based on cytoarchitecture.

Brain regions are connected to each other via long and short association tracts which are the neuroanatomical substrate of white matter. If early anatomical studies described the cerebral white matter as a uniform substance, in 1672 Willis used a scraping technique that was 
suggested by Nicolaus Steno to unravel the arrangement of medullary tracts within white matter. At a later stage, Theodor Hermann Meynert postulated the three principal types of white matter tracts:

1) the association tracts which link the different brain regions. These include both the short (the U-shaped fibers of Meynert) and the long association fibers

2) the commissural tracts that connect the two hemispheres

3) the afferent and efferent projection tracts between the cerebral cortex and subcortical structures.

Paul Emil Flechsig studied the myelogenesis of different cortical regions using myelin staining techniques (Flechsig, 1896). He found that different fiber tracts develop their myelin pattern at different periods during life and that tracts which belong to the same functional system develop at the same time (for a review, see: Schmahmann and Pandya, 2007).

Therefore, different brain regions are not connected in a random fashion. After Flechsig's work, the first attempt to link structure and function of the brain was undertaken by Campbell (Campbell, 1905). In his monograph, he combines patient studies with classical neuroanatomical staining techniques to unravel both the cyto- and the myeloarchitectural organization of the brain. This resulted in a map of 17 different cortical fields which were defined based on their functional role (ffytche and Catani, 2005).

The study of brain connectivity has kept developing and recently new tracing techniques have become available to trace white matter bundles which link different cortical regions. Invasive radioactive tracers have been used in several animal studies and are based on active transportation of a chemical compound along the axons. After injecting the tracer in a cortical or subcortical location, it starts diffusing actively towards the synaptic terminals (anterograde) or in the reverse direction 
(retrograde). These advanced techniques have allowed the detailed study of the hierarchical organization of the visual system in the monkey brain (Fig. 4; Felleman and Van Essen, 1991), with one important limitation, namely that the strength of the connection (the number of projecting axons) was still unknown. Further studies have been performed which developed databases of weighted regional connectivity indices, encoding connection strength, based on tracing studies in the whole monkey cortex (Bakker et al., 2012; Markov et al., 2011; Stephan et al., 2000). The addition of a weighting index, together with the direction of the connection, represents a fundamental step to unravel the hierarchical structure between functionally specialized brain areas (Markov and Kennedy, 2013). Furthermore, since interspecies studies represent a fundamental step to understand brain evolution and development, a translation of these connectivity profiles to the human brain has been attempted, which was based on macroanatomical landmarks and structural similarities between human and macaques (Bezgin et al., 2012; Kotter and Wanke, 2005).

The neuroscientific endeavor of mapping the whole brain connectivity map represents a fundamental effort to understand cognition (Bressler and Menon, 2010; Van Essen and Ugurbil, 2012). Several attempts to map the macroscopic structural connectivity pattern of the whole brain have been made through history using different techniques (see for review: Catani et al., 2013). To summarize all the results obtained by using different methods in a convenient way, the brain can be conceptualized as a set of nodes (cortical regions and subcortical nuclei) connected by different edges (the axons). Mathematically, a set of nodes and edges is called a graph and an entire branch of mathematics (graph theory) is dedicated to studying it. The graph representing all the cortico-cortical and cortico-subcortical connections between cortical areas and subcortical nuclei has been recently called the (macroscopic) connectome (Sporns et al., 2005). 


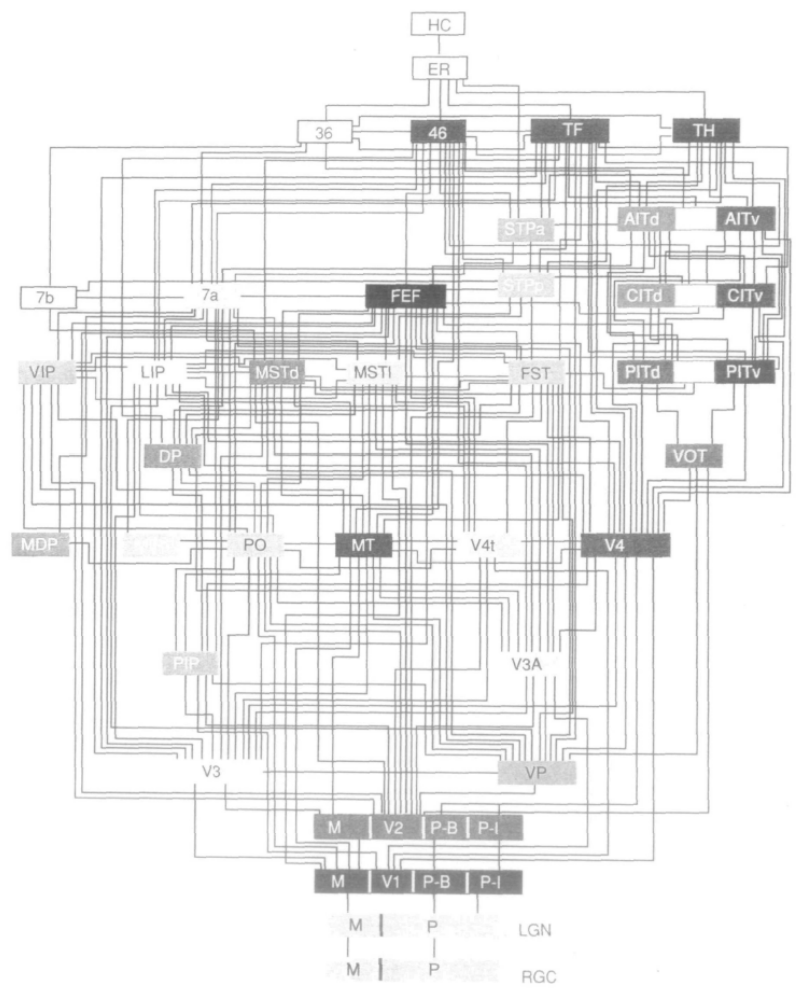

Figure 4: Organization of the visual system according to Felleman and Van Essen (1991). The scheme shows the pattern of hierarchical connectivity between different visual areas in the monkey brain.

\section{Mesoscopic brain organization: cortical columns and layers}

Zooming in to the mesoscopic scale the brain again offers very clear and coherent patterns of structural organization. From this viewpoint it is especially interesting to observe the architecture within gray matter. Roughly speaking, a grid-like structure can be defined. With respect to the pial surface, neurons in gray matter are organized radially (along the depth of the two to four millimeter thick cortex) in columns and tangentially (along the two dimension surface of the cortex) in layers. A single cortical column spans the whole cortical thickness, from the pial surface to white matter and, when combined with other columns, forms 
a so-called macrocolumn (Mountcastle, 1997). In contradistinction to layers, the definition of cortical columns is not solely anatomical. The definition of columns arose from electrophysiological studies reported that, when moving electrodes perpendicularly to the gray matter surface, there was a very strong degree of consistency in the receptive field of neurons (Hubel and Wiesel, 1962, 1968; Mountcastle, 1997). The laminar organization of the cortex is much more neuroanatomically defined. From the cytoarchitectural point of view, the cortex is organized in layers which are tangential to the pial surface and are characterized by different densities and sizes of cell bodies. In his original work focused on the cytoarchitecture of motor cortex, Santiago Ramon y Cajal described the presence of seven distinct layers (Ramón y Cajal, 1899). Nowadays the canonical mesoscopic representation of the neocortical gray matter comprises six different layers. This representation is based on the seminal work by Bevan Lewis (1880) which was then used by Korbinian Brodmann to parcellate the human cortical surface in fifty-two distinct regions (Brodmann, 1909).

Boundaries between different cortical regions can be identified based on the fact that, as already stated by Cajal, not all areas have the same number of layers. Furthermore, if the same layers are always present within a cortical patch, then area borders can be determined by comparing the difference in thickness between layers in different areas. As both cyto- and myeloarchitectonics show a layered organization of the cortical mantle, the delineation of cortical areas can be inferred from different patterns of cell bodies as well as from changes in intracortical fiber configurations. Within the cortex, myelin is present, wrapping axons in coherently organized fiber bundles mostly oriented in radial or tangential directions (for a review, see: Nieuwenhuys, 2013). This the reason that the differences in myelination between cortical regions can be used as a method to identify cortical boundaries (Annese et al., 2004; Sanides, 1962, 1964; Vogt, 1919; Vogt, 1910). In general, it was already agreed in the early $20^{\text {th }}$ century that cytoarchitectonic layers coincide 
with myeloarchitectonic layers, with the difference that the latter seem to show a higher and finer degree of differentiability in several sublayers (Fig. 5). However, the relationship between cytoarchitecture and myeloarchitecture in the cortex is still not clear. In his work, Hellwig (1993) showed, using simulations, that it is possible to reconstruct the myelin pattern starting from the cytoarchitectural pattern using two assumptions, namely that bigger neurons are more myelinated and that the distribution of axon collaterals can be quantified using a simple model.

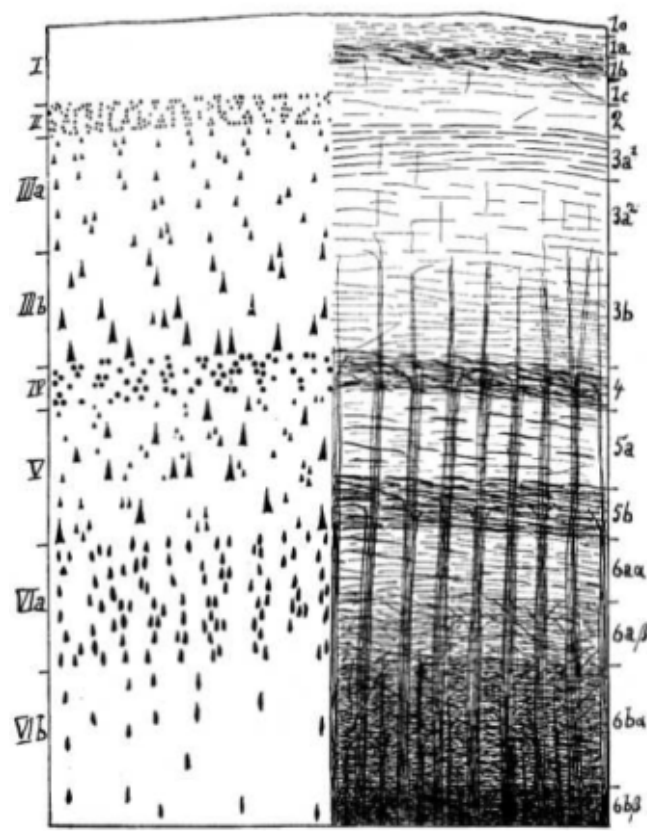

Figure 5: Vogt's (1910) basic scheme of cyto- and myeloarchitectural layering of the human neocortex.

Each cortical layer tends to be characterized by different afferent and efferent projections. There are three main patterns of laminar projections within the hierarchically organized cortical system, namely ascending, lateral and descending projections (Felleman and Van Essen, 1991). These pathways can have a unilaminar and/or a bilaminar origin. 
Ascending pathways coming from hierarchically lower cortical areas terminate in layer 4, lateral projections coming from hierarchically similar cortical areas terminate across all the different layers in a columnar fashion and descending projections coming from hierarchically higher cortical areas avoid layer 4 and terminate both in superficial and deep layers. Generally, infragranular layers (layers V and VI) are those that mainly project to sub-cortical areas, while supragranular layers (layers II and III) are connected to other cortical locations (Fuster, 2005; Jones, 1981). Finally, differences in laminar development can be observed across species, hinting to the fact that cortical columns could also differ across species due to evolution (Hutsler et al., 2005).

\section{Microscopic brain organization: axonal densities and diameters}

From the microscopic viewpoint, both white and gray matter show a wide range of different patterns in their structural organization. Within gray matter, cell bodies vary in size, shape and packing density. On the other hand, within white matter it is possible to find at least three distinct structural elements, namely axons, myelin and glial cells. In the latter class oligodendrocytes can be found; these are responsible for coating axonal membranes with myelin. Putting white matter under the microscope reveals a very coherent structure, which is mostly made up by parallel bundles of axons. Furthermore, axons are characterized by different diameters and their packing density changes across the white matter volume.

The corpus callosum is the biggest interhemispheric white matter commissure in the mammalian central nervous system. Within the corpus callosum of both macaque (LaMantia and Rakic, 1990) and human (Aboitiz et al., 1992), several clusters of tracts have been identified based on differences in axonal diameter distribution and 
packing density (Fig. 6). Using light microscopy, Aboitiz et al. (1992) examined the corpus callosum of several individuals and found that thin axons are denser in the genu. Furthermore, their density decreases moving towards the posterior midbody and increase again in the splenium. On the other hand, larger fibers show the opposite behavior, with a maximum density in the posterior midbody of the corpus callosum. Interestingly, they were not able to find any correlation between callosal volume and fiber density and between sex and density.

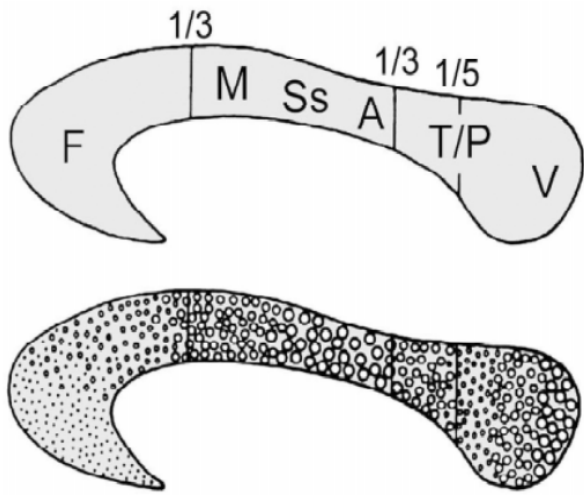

A

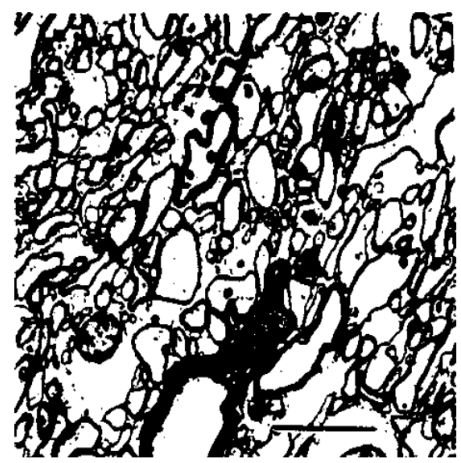

B

Figure 6: A) schematic diagram of the organization of human interhemispheric callosal fibers (top; F, frontal; $\mathrm{M}$, motor; Ss, somatosensory; $\mathrm{A}$, auditory; $\mathrm{T} / \mathrm{P}$, temporal/parietal; $\mathrm{V}$, visual) and distribution of axonal diameters in the human corpus callosum (bottom). From Aboitiz et al. (1992). B) electron micrograph slice of the midbody of the human corpus callosum where big axonal diameters are mostly present (bar $=10 \mu \mathrm{m})$. From Aboitiz et al. (2003).

Despite their microstructural heterogeneity, the pattern of callosal fiber diameters is well defined when related to the main fiber termination areas, as they show a clear clustered pattern (Fig. 2A). Notably, the microanatomical differences are not random, but can be related to the functional specialization of cortical areas. The underlying principle that justifies this structural-functional relationship is that there is a clear correlation between axonal diameter and conduction velocity 
of the electrical signals, i.e. bigger axons will propagate action potentials at greater velocity (Tasaki et al., 1943). Since this strongly links structure and function, studying the microstructural architecture of the brain can help to link different cognitive skills to anatomical characteristics and better understand their changes in disease and development. For instance, several cases have shown that, during development, abnormalities in the diameter distribution of the fibers within the corpus callosum led to diseases such as autism, dyslexia and schizophrenia (Hughes, 2007; Njiokiktjien et al., 1994; Rice and Barone, 2000). Axonal tract are also characterized by different degrees of myelination which changes throughout early brain development (Miller et al., 2012).

Myelin sheaths wrap the axons to electrically insulate some of their length in a relatively regular pattern. By doing so, saltatory transmission of the neuronal current is possible, speeding up the travelling of information in the nervous system. In this way, degree of myelination is the second important microanatomical mediator of conduction velocities. It was long held that the degree of myelination is constant over the entire length of an axon, giving it a single conduction velocity and single conduction delay given its length and myelination. However, in a recent study, Tomassy and colleagues (2014) showed that the distribution of myelin along the axon of a neocortical pyramidal neuron can change inside the cortex. This has interesting implications for theories about network integration in the nervous system. Myelin might not just serve as an electrical insulator, but such differences in length of unmyelinated sections within the same neuron can help to regulate diverse transmission delays of the neuronal signal (Fields, 2014).

Therefore, studying conduction velocities and signal transmission in the nervous system requires taking myelination profiles into account. To effectively do so, anatomists have been looking to the so-called g-ratio, which is the ratio between the membrane-tomembrane axonal diameter and the myelinated fiber diameter. Rushton 
(Rushton, 1951) was the first to describe a mathematical approach to define the optimal g-ratio in the nervous system, which predicted to be 0.6. His theoretical framework was solely based on the velocity of signal transmission, and recent studies have both challenged and improved this framework in the central nervous system of the rat (Chomiak and $\mathrm{Hu}, 2009)$. The possibility of estimating the g-ratio in vivo using MRIbased techniques has recently been investigated (Stikov et al., 2014). This could make the g-ratio an important marker for detecting and studying progression of diseases, axonal plasticity and development.

Studying the human nervous system from the microscopic viewpoint can help to understand important differences between individuals and how structure can influence function and vice versa. As recently shown by Zikopoulos and Barbas (2011), the comparison of different microstructural indices between individual axons of the frontal cortex of both human and non-human primates leads to the identification of several differences and similarities between the different species. Such findings are fundamental to follow and explain the evolutionary paths walked by different species and represent a necessary step to understand the neuroanatomical underpinning of neurological diseases.

\section{Diffusion MRI: modern neuroanatomy}

The development of MRI neuroimaging techniques has made it possible to reach sub-millimeter resolution to map both the anatomy and the function of the brain. However, standard MRI acquisitions based on T1 and T2 contrasts give only a partial description of the brain's microstructural composition. Moreover, these contrasts contain little information about the anatomical connectivity between different cortical regions. Diffusion MRI (dMRI) overcomes some of these limitations. DMRI encodes the displacement of water molecules within the different brain compartments to infer about its structural organization. 
Physical and imaging principles of diffusion MRI

Free-floating molecules in a gaseous or fluid medium display thermal motion in a random walk pattern, a behavior known as Brownian motion or passive diffusion. Einstein (1905) formalized this diffusion behavior in mathematical terms for randomly diffusing particles in an isotropic medium (i.e. free diffusion). Statistically, the displacement probability distribution within an isotropic medium can be modelled using a Gaussian distribution, with its variance and isoprobability contours moving outward with time. However, in the brain different cells, their membranes and organelles form biological compartments which constrain the displacement of water molecules, modifying their statistical behavior over time. Water diffusion measured over all but the shortest time intervals in biological tissue such as the brain is typically hindered or restricted by these barriers and anisotropic in nature.

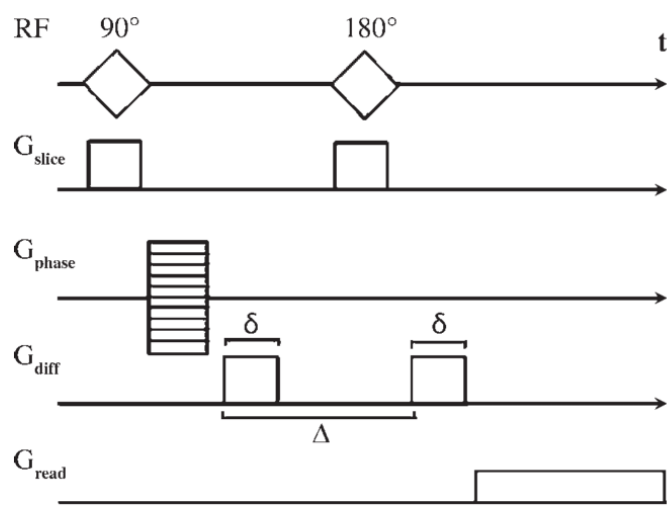

Figure 7: Schematic representation of the PGSE diffusion sequence. The figure has originally been published in Hagmann et al. (2006).

As such measuring water diffusion with dMRI characterizes the socalled apparent diffusion coefficient (ADC) dependent on not only on physical parameters but also on the parameters of the measurement. Brain white matter mostly consists of myelinated axons which are very 
coherently organized. In white matter there are two important microstructure compartments in which anisotropic water diffusion can be measured with dMRI: the intra-axonal and an extra-axonal compartment. Water diffusion within the confines of the axonal membrane is said to be restricted, while diffusion within the extracellular space is said to be hindered. The latter can be well approximated using an anisotropic Gaussian probability distribution, but for restricted diffusion the Gaussian assumption does not hold.

To measure the displacement of moving spins with dMRI, the pulsed gradient spin echo (PGSE) sequence is mostly used (Stejskal and Tanner, 1965). In this MRI pulse sequence, two high-amplitude diffusion gradients are applied to dephase and, after a spin-echo refocusing pulse has been delivered, rephase stationary spins (protons, Fig. 7). Spins that are moving along the direction of the applied diffusion gradient will show bulk dephasing and, consequently, signal loss. The signal loss is proportional to the area under the diffusion encoding gradients and to the time interval between the two gradients. In a mono-exponential signal decay model, the b-value is used as a measure of diffusion encoding:

$$
b=\gamma^{2} \delta^{2}|G|^{2}\left(\Delta-\frac{\delta}{3}\right)
$$

where $b$ is the $b$-value measured in $s / \mathrm{mm}^{2}, \gamma$ is the gyromagnetic ratio, $\delta$ is the duration of the diffusion pulse, $|G|$ is the amplitude of the diffusion gradients and $\Delta$ is the time interval between the two diffusion gradients (Fig. 7). The monoexponential approximation is valid at low bvalues and models the signal as:

$$
S_{i}=S_{0} e^{-b D_{i}}
$$

where $S_{i}$ is the measured signal with the $i^{\text {th }}$ diffusion encoding gradient in direction $\left[\mathrm{x}_{\mathrm{i}}, \mathrm{y}_{\mathrm{i}}, \mathrm{Z}_{\mathrm{i}}\right], \mathrm{S}_{0}$ is the measured signal without diffusion encoding, $\mathrm{b}$ is the $\mathrm{b}$-value and $\mathrm{D}_{\mathrm{i}}$ is the apparent diffusion coefficient (or ADC). Under the narrow-pulse approximation $(\delta \ll \Delta)$, the ADC Di can 
then be estimated in every voxel of the image and for every sampled diffusion direction $\left[\mathrm{x}_{\mathrm{i}}, \mathrm{y}_{\mathrm{i}}, \mathrm{z}_{\mathrm{i}}\right]$.

The diffusion gradients could be said to probe a threedimensional space. This space is called q-space, which is sampled in addition to the standard image k-space (Hagmann et al., 2006). Every sampled point can be represented using vector $\vec{q}$ :

$$
\vec{q}=\frac{\gamma \delta \vec{G}}{2 \pi}
$$

where $\vec{G}$ is the diffusion gradient vector. Q-space imaging consists in estimating the diffusion propagator, i.e. the probability distribution of water molecules' displacement, or its statistical moments, by sampling points in q-space and using the Fourier transform relationship between q-space and the diffusion propagator. Diffusion spectrum imaging (DSI; Wedeen et al., 2005) links the diffusion signal directly to the diffusion propagator by sampling the entire three dimensional q-space on a Cartesian grid and using discrete Fourier analysis.

\section{Diffusion tensor imaging and beyond}

Diffusion tensor imaging (DTI; Basser et al., 1994; Basser and Pierpaoli, 1996) was introduced to characterize diffusion in an anisotropic medium, such as brain white matter. DTI today still represents a clinical and research standard for analyzing dMRI data. By sampling at least six linearly independent diffusion encoding directions and a non-diffusion weighted volume, it is possible to model the diffusion behavior of water molecules using a rank-2 tensor representation. As DTI strongly relies on the Gaussian assumption of water diffusion, it is only capable of reconstructing one main direction of diffusion. Given the high degree of coherence of axonal bundles in white matter, it can be assumed that such direction will correspond to the orientation of the axons. Furthermore, several scalar indices can be estimated from the diffusion 
tensor. Mean diffusivity (MD) measures the average degree of water diffusion within a voxel, while fractional anisotropy (FA) measures the directional specificity of diffusion as obtained by DTI analysis. Both these indices have been widely used to study neurodevelopment (McKinstry et al., 2002), axonal integrity (Khayal et al., 2011), clinical conditions (for a review, see: Dong et al., 2004) and plasticity (for a review, see: Zatorre et al., 2012).

The main limitation of DTI is its incapability of resolving more than one fiber component in every voxel. Recently it has been shown that in the brain, at a nominal resolution of current day standard dMRI acquisition (2-3 mm isotropic voxels), more than $90 \%$ of voxels contains more than one fiber bundle (Jeurissen et al., 2013). Therefore, new models for dMRI data have been proposed based on high angular resolution diffusion imaging (HARDI) acquisitions. By probing a high number of diffusion encoding directions in q-space, it is possible to model non Gaussian effects in the reconstruction of ADC profiles (Alexander et al., 2002; Frank, 2002). Q-ball imaging (QBI; Tuch, 2004) was the first attempt to model multiple fiber components in a voxel. Using a completely model-free approach, QBI reconstructs the spin displacement orientation distribution function (ODF). Spherical deconvolution (Tournier et al., 2004) and, subsequently, constrained spherical deconvolution (Tournier et al., 2007), models the actual fiber orientation distribution (FOD) modelling complex fiber configurations as linear combinations of Dirac's delta functions. A Bayesian framework has also been proposed by which it is possible to fit an isotropic component and several fiber components to the sampled diffusion signal, with an estimate of directional uncertainty (Behrens et al., 2007). Moreover, a generalization of single shell (i.e. single b-value) HARDIbased techniques has been proposed. Generalized q-sampling imaging (GQI; Yeh et al., 2010) increases the sensitivity to multiple fiber compartments which are characterized by different microstructural properties by sampling q-space using multiple shells. 


\section{Fiber tracking}

Any set of modeled local orientations can be used for tractography or fiber tracking. That is, once the main orientation has been reconstructed in every voxel, it is possible to reconstruct by further modeling the association tracts connecting different brain regions. A way to do so is to use a streamline algorithm, which locally integrates the fiber path using a step-wise approach (Conturo et al., 1999; Mori et al., 1999; Mori and van Zijl, 2002). Based on post- mortem findings, in vivo virtual dissection of known fibers can be performed (Catani et al., 2002). Selecting two regions of interest (ROIs), through which the fiber tract should run, it is possible to reconstruct white matter tracts in vivo and non-invasively. The problem of spurious fibers can then be partially addressed by selecting a third exclusion ROI and remove the known false positives. Several other tractography approaches have been developed over the years which are capable of providing indices which reflect how strongly connected two regions are based on probabilistic frameworks (Parker et al., 2003) or on graph theory (Iturria-Medina et al., 2007; Iturria-Medina et al., 2008). Tractography algorithms have also been expanded to match the newly available information derived from HARDI-based techniques (Sotiropoulos et al., 2010; Wedeen et al., 2008). In chapter 2 of the present thesis we have addressed the issue related to the choice of a tractography algorithm and how such choice strongly influences the results.

\section{Macro, meso and microstructural mapping with diffusion MRI}

It is possible to map the macrostructural connection organization of the brain using dMRI and tractography in two different ways. First, when the starting and ending regions of tracts have been previously defined, tractography can be used to reconstruct the entire macroscopic wiring map of the brain, i.e. the macroscopic connectome, in vivo and noninvasively, the "connectome approach" (Hagmann et al., 2008; 
Sotiropoulos et al., 2013; Van Essen and Ugurbil, 2012). Secondly, the reverse approach is to define cortical areas using clustering approaches based on large scale brain structural connectivity profiles obtained using dMRI-based tractography, the "clustering approach" (Behrens et al., 2003; Mars et al., 2011; Perrin et al., 2008; Tomassini et al., 2007). In the connectome approach, the application of network analysis to the connectome graph has allowed neuroscientists to describe different properties of cortical areas based on their connectivity profiles, such as their degree of segregation based on clustering approaches or their centrality in the communication network (Rubinov and Sporns, 2010). In a recent paper, it has been shown that some cortical regions form a socalled 'rich club', in the sense that those areas are not only very central areas necessary for information processing (i.e. brain hubs), but are also very strongly connected to each other (van den Heuvel and Sporns, 2011). These findings can be then used to study several pathological conditions by bringing the analysis to a new level of abstraction (van den Heuvel et al., 2010), in which different brains can be compared based on their connectivity profiles rather than using a common standard template. The clustering approach has led to the identification and subdivision of several cortical (Mars et al., 2011; Perrin et al., 2008; Tomassini et al., 2007) and subcortical (Behrens et al., 2003) areas. More recently, the clustering of large parts or the entire human neocortex into areas has been attempted using this approach (Gorbach et al., 2011; Perrin et al., 2008).

Recently, it has been shown that dMRI can be used to map the mesoscopic organization of the brain. Post mortem sample imaging has recently progressed to studying the structural organization of the whole brain at a voxel resolution of $0.7 \mathrm{~mm}$ isotropic (Miller et al., 2011). Other works have focused on smaller tissue samples to reduce the necessary field of view and increase isotropic voxel size, bringing it to $0.2-0.4 \mathrm{~mm}$. In their paper, Leuze et al. (2014) describe the intra and inter-laminar connectivity within post mortem tissue samples of primary motor and 
somatosensory cortex using spherical deconvolution based tractography. Furthermore, the organization of primary visual cortex, where the highly myelinated stria of Gennari can be found, was investigated using post mortem dMRI (Kleinnijenhuis et al., 2013). In chapter 4 of this thesis post mortem high resolution dMRI is used to investigate the mesoscopic layered organization of motor and premotor cortex.

Finally, dMRI has proven to be successful in mapping brain microstructure by estimating axonal diameters and axonal density both in vivo and post mortem. New modelling approaches have been proposed that estimate and separate the contributions of different biological compartments within white matter, most importantly the restricted intra- and hindered extra-axonal compartments (Assaf and Basser, 2005; Panagiotaki et al., 2012; Zhang et al., 2012). If modeled correctly, it has been shown that these two separate water pools will be characterized by different signal profiles in standard pulsed gradient spin echo (PGSE) experiments (Stanisz et al., 1997). DMRI microstructural compartment models fit a model of white matter to the acquired data to infer the microstructural characteristics of the tissue from model parameters such as compartment volume fractions (for a review, see: Panagiotaki et al., 2012). This makes it possible to infer patterns of axonal densities in the human brain as well as relative axonal diameter distributions, both in vivo and ex-vivo (Alexander et al., 2010; Assaf et al., 2008; Barazany et al., 2009; Zhang et al., 2012).

\section{Research goals of the thesis}

The bases of the brain's structural organization from three different viewpoints were summarized in the introduction. Furthermore, dMRI was presented together with its capability to resolve the anatomy of the brain covering the entire spectrum of anatomical details. This thesis (Fig. 8) provides the neuroimaging community, which comprises both 
neuroscientists and clinicians, with novel tools and evidence to understand the structural architecture of the brain, its evolution and the anatomical underpinnings of functional specialization.

\begin{tabular}{ccc} 
Macroscale & Mesoscale & Microscale \\
$1000-3000 \mu \mathrm{m}$ & $10-100 \mu \mathrm{m}$ & $0.01-1 \mu \mathrm{m}$ \\
\hline
\end{tabular}

Long-range projections

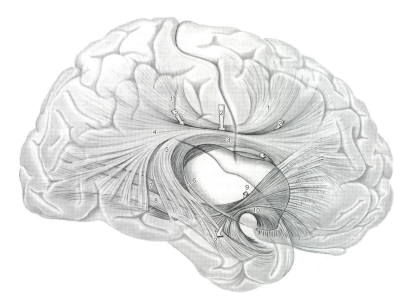

Intra-cortical organization

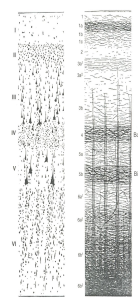

Axonal diameter and density

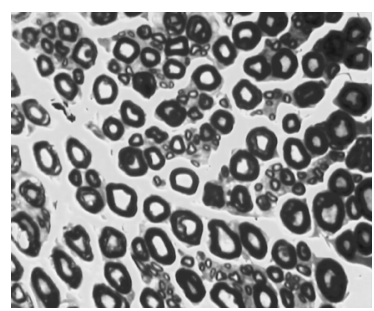

Chapter 2 and 3

Chapter 4

Chapter 5

Figure 8: Organization of the thesis according to the structural scale continuum.

Chapter 2 deals with the macrostructural connectivity profile of the human cortex as obtained by dMRI-based tractography. Several tractography approaches and two intra-voxel models of diffusion are used to compare different indices and hub profiles obtained by network analysis on the whole brain connectivity matrix. Furthermore, a novel framework is introduced to evaluate connectome reconstruction based on the tract specific density coefficient (TSDC).

In chapter 3 the connectome is used to compare the macaque and human structural architecture of the brain. The macaque connectome was obtained by using a database of tracing studies, while the human connectome was reconstructed using in vivo tractography. Using a regional map to identify 82 homologous regions between the two 
species, a quantitative comparison could be performed to describe evolutionary differences and similarities.

Chapter 4 shifts its focus to gray matter structure at the mesoscopic level. Using ultra-high field 9.4T preclinical scanner and a post-mortem human tissue sample it was possible to resolve cortical layers and areas in an automated and observer independent way using dMRI indices. A comparison with histological stains was also performed to validate the findings and correlate them with cyto and myeloarchitectural profiles.

The microstructural organization of the brain is the focus of chapter 5 . In this chapter the optimal scanning parameters are defined to obtain the best microstructural depiction of the brain using dMRI and novel acquisition techniques, together with a high amplitude gradient set on a 3T clinical scanner. Lastly, chapter 6 contains the summary and the discussion of the thesis. 


\section{References}

Aboitiz, F., Lopez, J., Montiel, J., 2003. Long distance communication in the human brain: timing constraints for inter-hemispheric synchrony and the origin of brain lateralization. Biol Res 36, 89-99.

Aboitiz, F., Scheibel, A.B., Fisher, R.S., Zaidel, E., 1992. Fiber composition of the human corpus callosum. Brain Research 598, 143-153.

Alexander, D.C., Barker, G.J., Arridge, S.R., 2002. Detection and modeling of nonGaussian apparent diffusion coefficient profiles in human brain data. Magn Reson Med $48,331-340$.

Alexander, D.C., Hubbard, P.L., Hall, M.G., Moore, E.A., Ptito, M., Parker, G.J., Dyrby, T.B., 2010. Orientationally invariant indices of axon diameter and density from diffusion MRI. Neuroimage 52, 1374-1389.

Amunts, K., Lenzen, M., Friederici, A.D., Schleicher, A., Morosan, P., PalomeroGallagher, N., Zilles, K., 2010. Broca's region: novel organizational principles and multiple receptor mapping. PLoS Biol 8.

Amunts, K., Lepage, C., Borgeat, L., Mohlberg, H., Dickscheid, T., Rousseau, M.E., Bludau, S., Bazin, P.L., Lewis, L.B., Oros-Peusquens, A.M., Shah, N.J., Lippert, T., Zilles, K., Evans, A.C., 2013. BigBrain: an ultrahigh-resolution 3D human brain model. Science $340,1472-1475$.

Annese, J., Pitiot, A., Dinov, I.D., Toga, A.W., 2004. A myelo-architectonic method for the structural classification of cortical areas. Neuroimage 21, 15-26.

Assaf, Y., Basser, P.J., 2005. Composite hindered and restricted model of diffusion (CHARMED) MR imaging of the human brain. Neuroimage 27, 48-58.

Assaf, Y., Blumenfeld-Katzir, T., Yovel, Y., Basser, P.J., 2008. AxCaliber: a method for measuring axon diameter distribution from diffusion MRI. Magn Reson Med 59, 13471354

Bakker, R., Wachtler, T., Diesmann, M., 2012. CoCoMac 2.0 and the future of tracttracing databases. Front Neuroinform 6, 30.

Barazany, D., Basser, P.J., Assaf, Y., 2009. In vivo measurement of axon diameter distribution in the corpus callosum of rat brain. Brain 132, 1210-1220.

Basser, P.J., Mattiello, J., LeBihan, D., 1994. MR diffusion tensor spectroscopy and imaging. Biophys J 66, 259-267.

Basser, P.J., Pierpaoli, C., 1996. Microstructural and physiological features of tissues elucidated by quantitative-diffusion-tensor MRI. J Magn Reson B 111, 209-219.

Behrens, T.E., Berg, H.J., Jbabdi, S., Rushworth, M.F., Woolrich, M.W., 2007. Probabilistic diffusion tractography with multiple fibre orientations: What can we gain? Neuroimage 34, 144-155. 
Behrens, T.E., Johansen-Berg, H., Woolrich, M.W., Smith, S.M., Wheeler-Kingshott, C.A., Boulby, P.A., Barker, G.J., Sillery, E.L., Sheehan, K., Ciccarelli, O., Thompson, A.J., Brady, J.M., Matthews, P.M., 2003. Non-invasive mapping of connections between human thalamus and cortex using diffusion imaging. Nat Neurosci 6, 750-757.

Bezgin, G., Vakorin, V.A., van Opstal, A.J., McIntosh, A.R., Bakker, R., 2012. Hundreds of brain maps in one atlas: registering coordinate-independent primate neuroanatomical data to a standard brain. Neuroimage 62, 67-76.

Bonin, G.v., Bailey, P., 1947. The neocortex of Macaca mulatta. University of Illinois Press, Urbana,.

Boucsein, C., Nawrot, M.P., Schnepel, P., Aertsen, A., 2011. Beyond the cortical column: abundance and physiology of horizontal connections imply a strong role for inputs from the surround. Front Neurosci 5, 32.

Bressler, S.L., Menon, V., 2010. Large-scale brain networks in cognition: emerging methods and principles. Trends Cogn Sci 14, 277-290.

Brodmann, K., 1909. Vergleichende Lokalisationslehre der Grosshirnrinde in ihren Prinzipien dargestellt auf Grund des Zellenbaues.

Campbell, A.W., 1905. Histological studies on the localisation of cerebral function, by Alfred W. Campbell ... Pub. by aid of a subsidy from the Royal Society of London. University Press, Cambridge.

Catani, M., Howard, R.J., Pajevic, S., Jones, D.K., 2002. Virtual in vivo interactive dissection of white matter fasciculi in the human brain. Neuroimage 17, 77-94.

Catani, M., Thiebaut de Schotten, M., Slater, D., Dell'Acqua, F., 2013. Connectomic approaches before the connectome. Neuroimage 80, 2-13.

Chenn, A., Walsh, C.A., 2002. Regulation of cerebral cortical size by control of cell cycle exit in neural precursors. Science 297, 365-369.

Chomiak, T., Hu, B., 2009. What Is the Optimal Value of the g-Ratio for Myelinated Fibers in the Rat CNS? A Theoretical Approach. Plos One 4.

Conturo, T.E., Lori, N.F., Cull, T.S., Akbudak, E., Snyder, A.Z., Shimony, J.S., McKinstry, R.C., Burton, H., Raichle, M.E., 1999. Tracking neuronal fiber pathways in the living human brain. Proc Natl Acad Sci U S A 96, 10422-10427.

Dong, Q., Welsh, R.C., Chenevert, T.L., Carlos, R.C., Maly-Sundgren, P., Gomez-Hassan, D.M., Mukherji, S.K., 2004. Clinical applications of diffusion tensor imaging. J Magn Reson Imaging 19, 6-18.

Eickhoff, S.B., Rottschy, C., Kujovic, M., Palomero-Gallagher, N., Zilles, K., 2008. Organizational Principles of Human Visual Cortex Revealed by Receptor Mapping. Cerebral Cortex 18, 2637-2645.

Einstein, A., 1905. Über die von der molekularkinetischen Theorie der Wärme geforderte Bewegung von in ruhenden Flüssigkeiten suspendierten Teilchen. Annalen der Physik 322, 549-560. 
Felleman, D.J., Van Essen, D.C., 1991. Distributed Hierarchical Processing in the Primate Cerebral Cortex. Cerebral Cortex 1, 1-47.

ffytche, D.H., Catani, M., 2005. Beyond localization: from hodology to function. Philos Trans R Soc Lond B Biol Sci 360, 767-779.

Fields, R.D., 2014. Myelin-More than Insulation. Science 344, 264-266.

Flechsig, P., 1896. Gehirn und seele. Veit \& comp., Leipzig,.

Frank, L.R., 2002. Characterization of anisotropy in high angular resolution diffusionweighted MRI. Magn Reson Med 47, 1083-1099.

Fuster, J.M., 2005. Neurobiology of Cortical Networks. Cortex and Mind. Oxford University Press, New York.

Geyer, S., Matelli, M., Luppino, G., Schleicher, A., Jansen, Y., Palomero-Gallagher, N., Zilles, K., 1998. Receptor autoradiographic mapping of the mesial motor and premotor cortex of the macaque monkey. Journal of Comparative Neurology 397, 231-250.

Gorbach, N.S., Schutte, C., Melzer, C., Goldau, M., Sujazow, O., Jitsev, J., Douglas, T., Tittgemeyer, M., 2011. Hierarchical information-based clustering for connectivity-based cortex parcellation. Front Neuroinform 5, 18.

Hagmann, P., Cammoun, L., Gigandet, X., Meuli, R., Honey, C.J., Wedeen, V.J., Sporns, O., 2008. Mapping the structural core of human cerebral cortex. PLoS Biol 6, e159.

Hagmann, P., Jonasson, L., Maeder, P., Thiran, J.P., Wedeen, V.J., Meuli, R., 2006. Understanding diffusion MR imaging techniques: from scalar diffusion-weighted imaging to diffusion tensor imaging and beyond. Radiographics 26 Suppl 1, S205-223.

Hellwig, B., 1993. How the myelin picture of the human cerebral cortex can be computed from cytoarchitectural data. A bridge between von Economo and Vogt. J Hirnforsch 34, 387-402.

Hilgetag, C.C., Barbas, H., 2005. Developmental mechanics of the primate cerebral cortex. Anat Embryol (Berl) 210, 411-417.

Hilgetag, C.C., Barbas, H., 2006. Role of mechanical factors in the morphology of the primate cerebral cortex. PLoS Comput Biol 2, e22.

Hodgkin, A.L., Huxley, A.F., 1952. A quantitative description of membrane current and its application to conduction and excitation in nerve. J Physiol 117, 500-544.

Hubel, D.H., Wiesel, T.N., 1962. Receptive fields, binocular interaction and functional architecture in the cat's visual cortex. J Physiol 160, 106-154.

Hubel, D.H., Wiesel, T.N., 1968. Receptive fields and functional architecture of monkey striate cortex. J Physiol 195, 215-243.

Hughes, J.R., 2007. Autism: the first firm finding = underconnectivity? Epilepsy Behav $11,20-24$. 
Hutsler, J.J., Lee, D.G., Porter, K.K., 2005. Comparative analysis of cortical layering and supragranular layer enlargement in rodent carnivore and primate species. Brain Research 1052, 71-81.

Iturria-Medina, Y., Canales-Rodriguez, E.J., Melie-Garcia, L., Valdes-Hernandez, P.A., Martinez-Montes, E., Aleman-Gomez, Y., Sanchez-Bornot, J.M., 2007. Characterizing brain anatomical connections using diffusion weighted MRI and graph theory. Neuroimage 36, 645-660.

Iturria-Medina, Y., Sotero, R.C., Canales-Rodriguez, E.J., Aleman-Gomez, Y., MelieGarcia, L., 2008. Studying the human brain anatomical network via diffusion-weighted MRI and Graph Theory. Neuroimage 40, 1064-1076.

Jeurissen, B., Leemans, A., Tournier, J.D., Jones, D.K., Sijbers, J., 2013. Investigating the prevalence of complex fiber configurations in white matter tissue with diffusion magnetic resonance imaging. Hum Brain Mapp 34, 2747-2766.

Jones, E.G., 1981. Anatomy of cerebral cortex: Columnar input-output organization. In: Schmitt, F.O., Worden, F.G., Adelman, G., Dennis, S.G. (Eds.), The organization of the cerebral cortex. MIT Press, Cambridge, Mass.

Khayal, I.S., Vandenberg, S.R., Smith, K.J., Cloyd, C.P., Chang, S.M., Cha, S., Nelson, S.J., McKnight, T.R., 2011. MRI apparent diffusion coefficient reflects histopathologic subtype, axonal disruption, and tumor fraction in diffuse-type grade II gliomas. Neuro Oncol 13, 1192-1201.

Kleinnijenhuis, M., Zerbi, V., Kusters, B., Slump, C.H., Barth, M., van Cappellen van Walsum, A.M., 2013. Layer-specific diffusion weighted imaging in human primary visual cortex in vitro. Cortex 49, 2569-2582.

Kotter, R., Wanke, E., 2005. Mapping brains without coordinates. Philosophical Transactions of the Royal Society B-Biological Sciences 360, 751-766.

LaMantia, A.S., Rakic, P., 1990. Cytological and quantitative characteristics of four cerebral commissures in the rhesus monkey. Journal of Comparative Neurology 291, 520-537.

Leuze, C.W., Anwander, A., Bazin, P.L., Dhital, B., Stuber, C., Reimann, K., Geyer, S., Turner, R., 2014. Layer-specific intracortical connectivity revealed with diffusion MRI. Cerebral Cortex 24, 328-339.

Lewis, W.B., 1880. Researches on the comparative structure of the cortex cerebri. Trübner \& Co., London.

Lui, J.H., Hansen, D.V., Kriegstein, A.R., 2011. Development and evolution of the human neocortex. Cell 146, 18-36.

Markov, N.T., Kennedy, H., 2013. The importance of being hierarchical. Current Opinion in Neurobiology 23, 187-194.

Markov, N.T., Misery, P., Falchier, A., Lamy, C., Vezoli, J., Quilodran, R., Gariel, M.A., Giroud, P., Ercsey-Ravasz, M., Pilaz, L.J., Huissoud, C., Barone, P., Dehay, C., Toroczkai, 
Z., Van Essen, D.C., Kennedy, H., Knoblauch, K., 2011. Weight Consistency Specifies Regularities of Macaque Cortical Networks. Cerebral Cortex 21, 1254-1272.

Mars, R.B., Jbabdi, S., Sallet, J., O'Reilly, J.X., Croxson, P.L., Olivier, E., Noonan, M.P., Bergmann, C., Mitchell, A.S., Baxter, M.G., Behrens, T.E., Johansen-Berg, H., Tomassini, V., Miller, K.L., Rushworth, M.F., 2011. Diffusion-weighted imaging tractography-based parcellation of the human parietal cortex and comparison with human and macaque resting-state functional connectivity. J Neurosci 31, 4087-4100.

McKinstry, R.C., Mathur, A., Miller, J.H., Ozcan, A., Snyder, A.Z., Schefft, G.L., Almli, C.R., Shiran, S.I., Conturo, T.E., Neil, J.J., 2002. Radial organization of developing preterm human cerebral cortex revealed by non-invasive water diffusion anisotropy MRI. Cerebral Cortex 12, 1237-1243.

Miller, D.J., Duka, T., Stimpson, C.D., Schapiro, S.J., Baze, W.B., McArthur, M.J., Fobbs, A.J., Sousa, A.M., Sestan, N., Wildman, D.E., Lipovich, L., Kuzawa, C.W., Hof, P.R., Sherwood, C.C., 2012. Prolonged myelination in human neocortical evolution. Proc Natl Acad Sci U S A 109, 16480-16485.

Miller, K.L., Stagg, C.J., Douaud, G., Jbabdi, S., Smith, S.M., Behrens, T.E., Jenkinson, M., Chance, S.A., Esiri, M.M., Voets, N.L., Jenkinson, N., Aziz, T.Z., Turner, M.R., JohansenBerg, H., McNab, J.A., 2011. Diffusion imaging of whole, post-mortem human brains on a clinical MRI scanner. Neuroimage 57, 167-181.

Morell, P., 1984. Myelin. New York and London: Plenum Press.

Mori, S., Crain, B.J., Chacko, V.P., van Zijl, P.C., 1999. Three-dimensional tracking of axonal projections in the brain by magnetic resonance imaging. Ann Neurol 45, 265-269.

Mori, S., van Zijl, P.C., 2002. Fiber tracking: principles and strategies - a technical review. NMR Biomed 15, 468-480.

Mountcastle, V.B., 1997. The columnar organization of the neocortex. Brain 120 ( Pt 4), 701-722.

Nieuwenhuys, R., 2013. The myeloarchitectonic studies on the human cerebral cortex of the Vogt-Vogt school, and their significance for the interpretation of functional neuroimaging data. Brain Struct Funct 218, 303-352.

Njiokiktjien, C., de Sonneville, L., Vaal, J., 1994. Callosal size in children with learning disabilities. Behav Brain Res 64, 213-218.

Panagiotaki, E., Schneider, T., Siow, B., Hall, M.G., Lythgoe, M.F., Alexander, D.C., 2012. Compartment models of the diffusion MR signal in brain white matter: a taxonomy and comparison. Neuroimage 59, 2241-2254.

Parker, G.J., Haroon, H.A., Wheeler-Kingshott, C.A., 2003. A framework for a streamline-based probabilistic index of connectivity (PICo) using a structural interpretation of MRI diffusion measurements. J Magn Reson Imaging 18, 242-254.

Perrin, M., Cointepas, Y., Cachia, A., Poupon, C., Thirion, B., Riviere, D., Cathier, P., El Kouby, V., Constantinesco, A., Le Bihan, D., Mangin, J.F., 2008. Connectivity-based 
parcellation of the cortical mantle using q-ball diffusion imaging. Int J Biomed Imaging $2008,368406$.

Ramón y Cajal, S., 1899. Estudios sobre la corteza cerebral humana. II. Estructura de la corteza motriz del hombre y mamiferos superiores. Revista trimestral Micrografica 4, $117 / 200$.

Rice, D., Barone, S., Jr., 2000. Critical periods of vulnerability for the developing nervous system: evidence from humans and animal models. Environ Health Perspect 108 Suppl 3, 511-533.

Rubinov, M., Sporns, O., 2010. Complex network measures of brain connectivity: uses and interpretations. Neuroimage 52, 1059-1069.

Rushton, W.A., 1951. A theory of the effects of fibre size in medullated nerve. J Physiol $115,101-122$.

Sanides, F., 1962. [Architectonics of the human frontal lobe of the brain. With a demonstration of the principles of its formation as a reflection of phylogenetic differentiation of the cerebral cortex]. Monogr Gesamtgeb Neurol Psychiatr 98, 1-201.

Sanides, F., 1964. The Cyto-Myeloarchitecture of the Human Frontal Lobe and Its Relation to Phylogenetic Differentiation of the Cerebral Cortex. J Hirnforsch 7, 269-282.

Schmahmann, J.D., Pandya, D.N., 2007. Cerebral white matter - Historical evolution of facts and notions concerning the organization of the fiber pathways of the brain. Journal of the History of the Neurosciences 16, 237-267.

Sotiropoulos, S.N., Bai, L., Morgan, P.S., Constantinescu, C.S., Tench, C.R., 2010. Brain tractography using Q-ball imaging and graph theory: Improved connectivities through fibre crossings via a model-based approach. Neuroimage 49, 2444-2456.

Sotiropoulos, S.N., Jbabdi, S., Xu, J., Andersson, J.L., Moeller, S., Auerbach, E.J., Glasser, M.F., Hernandez, M., Sapiro, G., Jenkinson, M., Feinberg, D.A., Yacoub, E., Lenglet, C., Van Essen, D.C., Ugurbil, K., Behrens, T.E., Consortium, W.U.-M.H., 2013. Advances in diffusion MRI acquisition and processing in the Human Connectome Project. Neuroimage 80, 125-143.

Sporns, O., Tononi, G., Kotter, R., 2005. The human connectome: A structural description of the human brain. PLoS Comput Biol 1, e42.

Stanisz, G.J., Szafer, A., Wright, G.A., Henkelman, R.M., 1997. An analytical model of restricted diffusion in bovine optic nerve. Magn Reson Med 37, 103-111.

Stejskal, E.O., Tanner, J.E., 1965. Spin Diffusion Measurements: Spin Echoes in the Presence of a Time-Dependent Field Gradient. The Journal of Chemical Physics 42, 288292.

Stephan, K.E., Zilles, K., Kotter, R., 2000. Coordinate-independent mapping of structural and functional data by objective relational transformation (ORT). Philosophical Transactions of the Royal Society of London Series B-Biological Sciences 355, 37-54. 
Stikov, N., Campbell, J., Boudreau, M., Narayanan, S., Stroh, T., Nuara, S., Novek, J., Frey, S., Ho, M.-K., Bedell, B., Pike, B., 2014. In vivo histology of the myelin g-ratio. Proceedings of the 2014 Organization for Human Brain Mapping annual meeting, Hamburg, Germany, p. 2249.

Tasaki, I., Ishii, F., Ito, H., 1943. On the relation between the conduction rate and fiber diameter and the internodal distance of the medullated nerve fiber. Japanese Journal of Medical Sciences 9, 189-199.

Tomassini, V., Jbabdi, S., Klein, J.C., Behrens, T.E., Pozzilli, C., Matthews, P.M., Rushworth, M.F., Johansen-Berg, H., 2007. Diffusion-weighted imaging tractographybased parcellation of the human lateral premotor cortex identifies dorsal and ventral subregions with anatomical and functional specializations. J Neurosci 27, 10259-10269.

Tomassy, G.S., Berger, D.R., Chen, H.H., Kasthuri, N., Hayworth, K.J., Vercelli, A., Seung, H.S., Lichtman, J.W., Arlotta, P., 2014. Distinct profiles of myelin distribution along single axons of pyramidal neurons in the neocortex. Science 344, 319-324.

Tournier, J.D., Calamante, F., Connelly, A., 2007. Robust determination of the fibre orientation distribution in diffusion MRI: non-negativity constrained super-resolved spherical deconvolution. Neuroimage 35, 1459-1472.

Tournier, J.D., Calamante, F., Gadian, D.G., Connelly, A., 2004. Direct estimation of the fiber orientation density function from diffusion-weighted MRI data using spherical deconvolution. Neuroimage 23, 1176-1185.

Tuch, D.S., 2004. Q-ball imaging. Magn Reson Med 52, 1358-1372.

van den Heuvel, M.P., Mandl, R.C., Stam, C.J., Kahn, R.S., Hulshoff Pol, H.E., 2010. Aberrant frontal and temporal complex network structure in schizophrenia: a graph theoretical analysis. J Neurosci 30, 15915-15926.

van den Heuvel, M.P., Sporns, O., 2011. Rich-club organization of the human connectome. J Neurosci 31, 15775-15786.

Van Essen, D.C., 1997. A tension-based theory of morphogenesis and compact wiring in the central nervous system. Nature 385, 313-318.

Van Essen, D.C., Ugurbil, K., 2012. The future of the human connectome. Neuroimage $62,1299-1310$.

Vogt, C.V.O., 1919. Allgemeine Ergebnisse unserer Hirnforschung. J.A. Barth, Leipzig.

Vogt, O., 1910. Die myeloarchitektonische felderung des menschlichen stirnhirns. J Psychol Neurol 15, 221-232.

von Economo, C., Koskinas, G.N., 1925. Die Cytoarchitektonik der Hirnrinde des erwachsenen Menschen. Julius Springer, Vienna.

Wedeen, V.J., Hagmann, P., Tseng, W.Y., Reese, T.G., Weisskoff, R.M., 2005. Mapping complex tissue architecture with diffusion spectrum magnetic resonance imaging. Magn Reson Med 54, 1377-1386. 
Wedeen, V.J., Wang, R.P., Schmahmann, J.D., Benner, T., Tseng, W.Y., Dai, G., Pandya, D.N., Hagmann, P., D'Arceuil, H., de Crespigny, A.J., 2008. Diffusion spectrum magnetic resonance imaging (DSI) tractography of crossing fibers. Neuroimage 41, 1267-1277.

Yeh, F.C., Wedeen, V.J., Tseng, W.Y., 2010. Generalized q-sampling imaging. IEEE Trans Med Imaging 29, 1626-1635.

Zatorre, R.J., Fields, R.D., Johansen-Berg, H., 2012. Plasticity in gray and white: neuroimaging changes in brain structure during learning. Nat Neurosci 15, 528-536.

Zhang, H., Schneider, T., Wheeler-Kingshott, C.A., Alexander, D.C., 2012. NODDI: practical in vivo neurite orientation dispersion and density imaging of the human brain. Neuroimage 61, 1000-1016.

Zikopoulos, B., Barbas, H., 2011. Comparable organization of frontal white matter in human and non-human primates. Society For Neuroscience, Washington, DC.

Zilles, K., Amunts, K., 2009. Receptor mapping: architecture of the human cerebral cortex. Current Opinion in Neurology 22, 331-339.

Zilles, K., Amunts, K., 2010. TIMELINE Centenary of Brodmann's map - conception and fate. Nature Reviews Neuroscience 11, 139-145.

Zilles, K., Armstrong, E., Schleicher, A., Kretschmann, H.J., 1988. The human pattern of gyrification in the cerebral cortex. Anat Embryol (Berl) 179, 173-179.

Zilles, K., Palomero-Gallagher, N., Amunts, K., 2013. Development of cortical folding during evolution and ontogeny. Trends Neurosci 36, 275-284. 
Chapter 2

\section{Human cortical connectome reconstruction from diffusion weighted MRI: the effect of tractography algorithm}

Corresponding publication:

Bastiani, M., Shah, N.J., Goebel, R. and Roebroeck, A. (2012). Human cortical connectome reconstruction from diffusion weighted MRI: The effect of tractography algorithm. NeuroImage, 62(3), 1732-1749. 


\section{Abstract}

Reconstructing the macroscopic human cortical connectome by Diffusion Weighted Imaging (DWI) is a challenging research topic that has recently gained a lot of attention. In the present work, we investigate the effects of intra-voxel fiber direction modeling and tractography algorithm on derived structural network indices (e.g. density, small-worldness and global efficiency). The investigation is centered on three semi-independent distinctions within the large set of available diffusion models and tractography methods: i) single fiber direction versus multiple directions in the intra-voxel diffusion model, ii) deterministic versus probabilistic tractography and iii) local versus global measure-of-fit of the reconstructed fiber trajectories. The effect of algorithm and parameter choice has two components. First, there is the large effect of tractography algorithm and parameters on global network density, which is known to strongly affect graph indices. Second, and more importantly, there are remaining effects on graph indices which range in the tens of percent even when global density is controlled for. This is crucial for the sensitivity of any human structural network study and for the validity of study comparisons. We then investigate the effect of the choice of tractography algorithm on sensitivity and specificity of the resulting connections with a connectome dissection quality control (QC) approach. In this approach, evaluation of Tract Specific Density Coefficients (TSDCs) measures sensitivity while careful inspection of tractography path results assesses specificity. We use this to discuss interactions in the combined effects of these methods and implications for future studies. 


\section{Introduction}

Reconstructing in vivo the macro-scale human connectome (Sporns et al., 2005), which is the map of all the structural connections in the human brain, represents a challenging and important research topic in neuroimaging which has gained a lot of popularity in recent years. An individual large-scale connectome is represented by a graph with a set of nodes, representing a parcellation of the brain anatomy, and a set of edges (equivalently: an edge-weight connectivity matrix or binary adjacency matrix) which can be usefully analyzed using graph theory and network analysis (Rubinov and Sporns, 2010). The main technique by which individual structural connectome reconstruction is made possible in vivo is diffusion-weighted magnetic resonance imaging (DWI).

There are several steps in the post-processing pipeline for DWI data to create a connectivity matrix that represents the macroscopic structural connectome (Hagmann et al., 2007 and Hagmann et al., 2008). Among them are white matter and cortical gray matter segmentation from an anatomical (T1-weighted)MRI, parcellation of the gray matter into nodes of the connectome graph, creation of white matter seed points and masks, intra-voxel water diffusion and/or fiber direction modeling, tractography to connect local directions into long-distance connections and computation of edge-weights between cortical nodes from tractography results. Earlier work has investigated the effects of gray matter parcellation (Zalesky et al., 2010), seed point placement strategy for tractography (Cheng et al., 2011 and Li et al., 2011) and the reproducibility of network indices (Bassett et al., 2011a). For instance, Zalesky et al. (2010) show that the small-worldness measure increases with a larger number of nodes resulting from a greater degree of parcellation of the gray matter. In Li et al. (2011), it is shown that the identification of network hubs in the brain can depend on the tractography seed point placement strategy. 
Here we focus on the effects of intra-voxel fiber direction modeling and tractography algorithm on connectome graphs, each of which has seen intensive development and a plethora of approaches over the last decade. We center this investigation on three semiindependent distinctions within this large set of available methods, as summarized in Table 1: i) a single fiber direction compartment versus multiple directions in the intra-voxel diffusion model, ii) a deterministic versus a probabilistic tractography algorithm and iii) a local or step-wise versus global measure-of-fit to the voxel-wise data in reconstructing fiber trajectories.

Several models have been proposed to characterize intra-voxel water diffusion hindered or restricted by white matter fiber tissue, based on the diffusion-weighted MR signal. Diffusion tensor imaging (DTI; Basser and Pierpaoli, 1996 and Basser et al., 1994) represents the most established post-processing technique for DWI data. It consists of fitting a rank-2 tensor in each imaging voxel to model the molecules' diffusion as a three dimensional Gaussian probability distribution function (PDF). The uni-modal Gaussian PDF admits only a single main direction of diffusion in a voxel.

It was recognized early on that being able to estimate a single fiber direction per imaging voxel might not be enough. This is because partial volume effects will average two or more fiber populations with different local orientations in large parts of the imaging volume. Furthermore, fibers might be tightly interdigitated, producing something more like a 3-dimensional weave than multiple tight fasciculations. This has stimulated investigation into new acquisition protocols, such as high angular resolution diffusion imaging (HARDI; Frank, 2001 and Tuch et al., 2002), and new PDF reconstruction strategies that can produce multi-modal functions. Initially diffusion spectrum imaging (DSI; Wedeen et al., 2005) and Q-ball imaging (QBI; Tuch, 2004 and Tuch et al., 2003) were posited as techniques that estimate the actual spin displacement of water molecules without 
making any prior assumption about the shape of the 3D PDF. A crucial abstraction made in QBI was to model only the 2D orientation distribution function (ODF) on the sphere. The identification of more than one diffusion direction in a voxel enables superior fiber tractographythrough regions with multiple populations of fibers with different orientations (Wedeen et al., 2008). Subsequently, many more methods have been proposed to identify multiple fiber directions per voxel, including constrained spherical deconvolution (CSD; Tournier et al., 2004 and Tournier et al., 2007), the extended ball and stick model (Behrens et al., 2007), the diffusion orientation transform (DOT; Ozarslan et al., 2006), the composite hindered and restricted model of diffusion (CHARMED; Assaf and Basser, 2005), generalized diffusion tensors (Liu et al., 2004 and Ozarslan and Mareci, 2003) and generalized q-sample imaging (GQI; Yeh et al., 2010).

Once the fiber directions have been estimated for each voxel, tractography algorithms aim to reconstruct the axonal fiber bundles connecting different brain areas. The first tractography approaches to be developed connect discrete steps along the preferential diffusion directions (Basser et al., 2000, Conturo et al., 1999 and Mori et al., 1999), the so-called deterministic 'streamline' approach. So-called tensor deflection or tensor projection was introduced to use more than just the primary eigenvector of the local diffusion tensor model (Lazar et al., 2003 and Westin et al., 2002). The streamline approach was soon generalized to multi-direction fiber models (Tuch et al., 2003).

Probabilistic tractography approaches characterize the variability of tractography results arising from the uncertainty of the estimated local fiber directions (Behrens et al., 2003 and Parker et al., 2003). This contrasts them with deterministic approaches which give a single deterministic answer for the connection of a given region-of-interest (ROI) to any part of the brain. More precisely, the result of a probabilistic tractography algorithm for every single well-defined seed point is a $3 \mathrm{D}$ map of visitation counts for fibers through a voxel. The 
same noisy streamline or diffusing particle principles have been applied to multi-direction models to create what we call local probabilistic multi-direction approaches (Behrens et al., 2007,Parker and Alexander, 2005 and Tournier et al., 2010).

A final recent development step is to move from local step-wise reconstruction of fiber trajectories to a global goodness-of-fit of the entire candidate fiber. Here, the measure of fit quantifies the joint likelihood of the fiber given all voxel data it passes through (Jbabdi et al., 2007, Sherbondy et al., 2008, Sherbondy et al., 2009, Tuch et al., 2002 and Zalesky and Fornito, 2009). The global fit measure makes tractography less sensitive to modeling errors caused by local noise (Jbabdi et al., 2007). Recently, a graph-based tractography algorithm (Iturria-Medina et al., 2007) and its extension to a multiple direction fiber models (Sotiropoulos et al., 2010) have been proposed. These algorithms reconceptualize the global tractography problem as a shortest-path search in a graph, in which (in contrast with the connectome graph, described above) nodes are represented by the center of each white-matter voxel. Since graph-weights are then defined as the probability of voxel-center connections given the local ODFs, a shortest path from one point to another in this graph constitutes a globally optimized fiber. Since in a shortest-path search all possible nodes are visited and the path lengths recorded, the $\mathrm{n} \%$ shortest paths then correspond to the $\mathrm{n} \%$ most likely paths in the probabilistic tractography sense. Thus, graph-based methods - and in fact, global methods in general - are naturally used as probabilistic methods. The only way to force a global graph based approach to be deterministic is to select only its highest percentile results for consideration, which corresponds to looking at only the single shortest path that connects two points. This is why in Table 1, global methods are considered to be probabilistic, with the possibility of a deterministic interpretation when only the highest percentile results are considered. 


\begin{tabular}{|c|c|c|c|c|}
\hline \multirow{2}{*}{$\begin{array}{l}\text { Diffusion } \\
\text { model } \\
\text { Tractography } \\
\text { method }\end{array}$} & \multicolumn{2}{|c|}{ Single-direction } & \multicolumn{2}{|c|}{ Multi-direction } \\
\hline & Deterministic & Probabilistic & Deterministic & Probabilistic \\
\hline \multirow{4}{*}{$\begin{array}{l}\text { Local } \\
\text { tractography }\end{array}$} & SDD & SDP & MDD & MDP \\
\hline & $\begin{array}{l}\text { - DTI } \\
\text { streamline } \\
{[1]}\end{array}$ & \multirow{3}{*}{$\begin{array}{l}\text { - DTI } \\
\text { probabilistic } \\
\text { streamline } \\
{[4,5]}\end{array}$} & $\begin{array}{l}\text { - ODF } \\
\text { streamline } \\
{[6,7,8]}\end{array}$ & $\begin{array}{l}\text { - CSD } \\
\text { probabilistic } \\
\text { streamline }[10]\end{array}$ \\
\hline & \multirow[t]{2}{*}{$\begin{array}{l}\text { - Tensor } \\
\text { deflection [2, } \\
3]\end{array}$} & & $\begin{array}{l}\text { - CSD } \\
\text { streamline } \\
{[9]}\end{array}$ & $\begin{array}{l}\text { - Ball-and-stick } \\
\text { probabilistic } \\
\text { streamline [11] }\end{array}$ \\
\hline & & & & $\begin{array}{l}\text { - PAS } \\
\text { probabilistic } \\
\text { streamline [12] }\end{array}$ \\
\hline \multirow{3}{*}{$\begin{array}{l}\text { Global } \\
\text { tractography }\end{array}$} & \multicolumn{2}{|l|}{ SDG } & \multicolumn{2}{|l|}{ MDG } \\
\hline & \multicolumn{2}{|c|}{$\begin{array}{l}\text { - DTI Graph-tractography } \\
\text { [13] }\end{array}$} & \multicolumn{2}{|c|}{$\begin{array}{l}\text { - CSD Multigraph- tractography } \\
\text { [15] }\end{array}$} \\
\hline & \multicolumn{2}{|c|}{$\begin{array}{l}\text { - DTI streamline sampling and } \\
\text { scoring[14] }\end{array}$} & \multicolumn{2}{|c|}{$\begin{array}{l}\text { - ODF tracts sampling and scoring } \\
{[16]}\end{array}$} \\
\hline
\end{tabular}

Table 1: Different classes of intra-voxel diffusion models and tractography algorithms used in this work divided according to the dimensions: single-direction vs. multidirection intra-voxel diffusion model, deterministic vs. probabilistic tractography and local vs. global tractography. Examples within each category are listed, with the representative technique used in this study in boldface font. See main text for further explanation of specific methods and abbreviations. Tractography method class abbreviations: Single-direction deterministic (SDD), Single-direction probabilistic (SDP), Multiple-direction deterministic (MDD), Multiple-direction probabilistic (MDP), Singledirection global (SDG), Multiple-direction global (MDG). References: [1] (Basser et al., 2000), [2] (Westin et al., 2002), [3] (Lazar et al., 2003), [4] (Parker et al., 2003), [5] (Behrens et al., 2003), [6] (Tuch et al., 2003), [7] (Wedeen et al., 2008), [8] (Hagmann et al., 2008), [9] (Tournier et al., 2007), [10] (Tournier et al., 2010), [11] (Behrens et al., 2007), [12] (Parker and Alexander, 2005), [13] (Iturria-Medina et al., 2007), [14] (Sherbondy et al., 2008), [15] (Sotiropoulos et al., 2010), [16] (Sherbondy et al., 2009).

The first aim of this paper is assessing the effects of two different intra-voxel diffusion model classes and four different tractography algorithm classes (see Table 1) on the whole-brain connectivity matrix. 
To this end we evaluate the outcome of applying all method combinations to construct a cortical connectivity matrix. Commonly used graph measures are then calculated to evaluate their sensitivity to the employed diffusion model and tractography algorithm. This means that the question on which tractography approach to choose for in vivo connectome reconstruction becomes even more important. Therefore, in the second part of this paper we present a connectome dissection quality control (QC) approach that combines careful inspection of tractography path results with tract specific density coefficients (TSDCs) that quantify sensitivity and specificity.

\section{Materials and Methods}

\section{$M R$ data acquisition}

Whole brain scans of two healthy male subjects (aged 25 and 29) were acquired after obtaining written informed consent. Previous investigations have shown that the reproducibility of structural network indices over different subjects is very high (Bassett et al., 2011a). In addition, we investigate intra-subject reliability by computing 10 different random surface parcellations (Zalesky et al., 2010) for each subject (described below). The experiment was approved by the local ethical committee of the Faculty of Psychology and Neuroscience.

A Siemens 3 T MAGNETOM Allegra MR scanner equipped with a high slew-rate head gradient-coil (amplitude $40 \mathrm{mT} / \mathrm{m}$, slew rate $400 \mathrm{~T} / \mathrm{m} / \mathrm{s}$ ) and an 8-channel phased-array head RF-coil was used to acquire the data. A double refocused spin-echo diffusion sequence was used to acquire 131 volumes of data, with TR $=6600 \mathrm{~ms}$, TE $=94 \mathrm{~ms}$, bvalue $=3000 \mathrm{~s} / \mathrm{mm} 2,88 \times 88$ matrix, 52 axial slices, $2.5 \times 2.5 \times 2.5 \mathrm{~mm}^{3}$ voxels, partial Fourier $=6 / 8$ and a bandwidth of $2840 \mathrm{~Hz}$ /pixel (echospacing $0.4 \mathrm{~ms}$ ). A total of 120 diffusion gradient directions were acquired with 11 unweighted $(b=0 \mathrm{~s} / \mathrm{mm} 2)$ volumes acquired after 
every 12 gradient directions and including the first and last volumes. The total acquisition time for the diffusion weighted data was 14:30 min.

A T1-weighted 3D MPRAGE scan $(\mathrm{TR}=2250, \mathrm{TE}=2.6 \mathrm{~ms}$, flip angle $=9^{\circ}, 256 \times 256$ matrix, 192 sagittal slices, $1 \times 1 \times 1 \mathrm{~mm}$ voxels) was acquired for gray/white matter boundary segmentation. Resting statefMRI data was also acquired in the same session (not reported here). The subject's head was immobilized using foam cushions.

\section{Diffusion data preprocessing}

Diffusion data were preprocessed in order to correct for bulk motion and distortions induced by eddy currents using the FMRIB's Diffusion Toolbox available in FSL (Behrens et al., 2003). The estimated transformation matrices were used to transform the diffusion gradient directions accordingly (Leemans and Jones, 2009). No further transformations were performed on the diffusion-weighted data (or on the diffusion models estimated from it, see below). That is: all directional modeling and tractography were performed in 'native' diffusion data space.

All algorithms use the same white matter masks or waypoint maps, through which the fibers were allowed to pass. In order to obtain these maps, fractional anisotropy (FA) volumes were thresholded using two values and a $3 \times 3 \times 3$ median filter was applied to remove any holes. To investigate the effect of FA threshold, two versions were created at values of 0.1 and 0.2 .

In the current study, we did not correct for EPI distortions by a field map correction. In this head scanner with high slew rate capability, the geometric distortion for diffusion imaging from EPI was found to be relatively small compared to $2.5 \mathrm{~mm}$ isotropic voxels. Field map correction was evaluated but was found not to lead to a significant improvement in distortions. 


\section{Anatomical data processing}

White matter (WM), gray matter (GM) and cerebrospinal fluid (CSF) were segmented using FSL FAST (Zhang et al., 2001). The white matter volume was then imported in BrainVoyager QX (v. 2.2; Goebel et al., 2006) for further refinement and creation of a 100,000 vertex tessellation of the WM/GM boundary of both hemispheres using the surface reconstruction algorithm. The boundary surface was transformed to the DWI data space by an affine transform to determine the termination point of white matter fibers at the gray matter boundary. By moving inward from the WM/GM boundary along the surface normal to the closest WM mask voxel, 30.000 unique WM/GM boundary (WMbound) voxels were identified in diffusion data space. These Wmbound voxels were considered as termination loci when reconstructing the high-resolution connectome.

A lower-resolution connectome was obtained from the highresolution one by parcellating the WM/GM surface in 4000 random equi-surface patches (the connectome graph nodes) using a modified iterative constrained centroidal Voronoi tessellation (CCVT) approach (Du et al., 2003) which is described in more detail in Appendix A. The number of 4000 was motivated by Zalesky et al. (2010) and the aim to use a large number of equi-surface parcels that simultaneously minimizes the effect of parcellation and maximizes the sensitivity to the effects of tractography approach. To further decrease the effect of any single random parcellation all results presented are averaged over 10 different random parcellations per subject. Standard errors are computed over all 10 parcellations and over both subjects.

\section{Voxel-wise diffusion model estimation}

In this paper, two models representing two general classes of intra-voxel diffusion and fiber architecture were used. As a single-direction model, diffusion tensors (DTs) were fitted to the acquired data by linear regression using a least-square minimization approach. The tensorial 
matrix was then diagonalized by a Jacobi rotation technique to obtain its orthogonal eigenvector system.

As a multi-direction HARDI based model, CSD fiber orientation distributions (FODs) were reconstructed (Tournier et al., 2007) over a five-fold tessellated icosahedron. This technique was selected for its robustness in estimating orientational distributions from HARDI data. Moreover, FODs represent actual fiber orientation distributions rather than water-bound spin displacements, which leads to stable and accurate local orientations that are very beneficial for both local and global tractography purposes (Fillard et al., 2011). Subsequently, up to three rank-1 6th-order tensors were fitted to every FOD (Schultz and Seidel, 2008) in order to extract up to three main fiber directions in each voxel. This technique allows estimation of the actual fiber orientations without directional bias from the spherical tessellation used when estimating the FODs.

All algorithms have been implemented in custom-written $\mathrm{C}++$ code and in MATLAB (R2009b, The MathWorks, Natick, Massachusetts, USA).

\section{Tractography algorithms and parameters}

To evaluate the different algorithm performances and the changes in the reconstructed connectivity matrices, two parameters were varied: FA and maximum angle (minimum radius of curvature) between two consecutive steps. The step size was set to $1 \mathrm{~mm}$ for all local tractography methods, while for the graph-based global algorithms the step size varies according to the neighborhood size used which, in this work, has been set to $5 \times 5 \times 5$ voxels (see below).

Two angular thresholds were used in this work: $30^{\circ}$ and $90^{\circ}$ which cover the extremes of a realistic range. Thirty degrees represents a very common choice in the diffusion imaging literature, especially when using deterministic algorithms. The value of $90^{\circ}$ widens the possibilities, allowing fibers to turn at very sharp angles. Given the 
anatomical variability, signal-to-noise ratio (SNR) and the size of the imaging voxels with respect to white matter microstructure, it is difficult to justify any specific angle threshold on a neuro-anatomical basis. Therefore, since this parameter is almost ubiquitously used in tractography, it is important to explore the effect of two extreme values.

FA maps were thresholded at two different values which are commonly used in the literature (0.1 and 0.2$)$ in order to obtain two different WM waypoint masks. These are binary masks containing only those voxels where fibers are allowed to propagate. Since all the tractography algorithms investigated in the present study were run in original diffusion data space, we have chosen to use median filtered FA masks computed in that same space, instead of white matter masks obtained from T1-weighted volumes segmentation, in order to achieve maximum integrity and alignment of WM masks to the diffusion data. To avoid influences on FA such as partial volume effects at the WM/GM boundary and in those voxels where more than two diffusion directions are reconstructed a 3-dimensional median filter has been applied to the thresholded WM volumes to fill holes in the masks. Furthermore, in the WM binary masks, WMbound voxels were always included in the volume after having thresholded the FA mask and used the median filter. Since surface parcellation is performed in anatomical space, alignment is still needed to define WMbound voxels in diffusion space. However, this procedure is slightly more tolerant of misalignment and distortions due to susceptibility gradients since we coregister the surface points from anatomical space to diffusion space by inward projection along the surface normals.

Local deterministic algorithms were started both from a Cartesian $3 \times 3 \times 3$ grid of seed points $(0.83 \mathrm{~mm}$ spacing $)$ in the interior volume of every WM mask voxel and from those voxels which only belong to the WMbound. All resulting tractography streamlines were then investigated for intersection, on two opposite sides of the seed point, with the WM boundary volume (i.e. all WMbound voxels). All 
probabilistic methods and global methods were started from all WM boundary voxels, which is necessary for the resulting visitation maps to be interpreted as connecting seed points at the WM/GM boundary to other WM/GM boundary points. In addition, when individual probabilistic streamlines are investigated rather than visitation maps, $\mathrm{Li}$ et al. (2011) show that distance related bias can increase when seeding from the entire deep white matter.

For all local methods, deterministic or probabilistic, fibers shorter than $10 \mathrm{~mm}$ or longer than $200 \mathrm{~mm}$ were removed. Furthermore, looping fibers (i.e. fibers that return to already explored voxels) are excluded from the analysis. For global algorithms by the very nature of the shortest path finding approach, loops are automatically excluded.

\section{Local single-direction deterministic (SDD)}

Deterministic streamlines were obtained using Euler stepping integration of the local direction field. Local direction finding takes into account the first eigenvector based on the estimated rank-2 tensor. Nearest neighbor interpolation of the entire diffusion tensor was performed. At every starting seed point, a streamline was initiated parallel to the local primary eigenvector. Deterministic local singledirection tractography was performed with custom-written $\mathrm{C}++$ code (c.f. Roebroeck et al., 2008).

\section{Local single-direction probabilistic (SDP)}

The local streamline-based approach to compute probabilistic indices of connectivity (PICo) as proposed by Parker et al. (2003) was used. This approach estimates the uncertainty in the diffusion direction estimation obtained by DTI and computes the connectivity strength between two regions using Monte Carlo methods. The number of iterations, which is the number of streamlines initiated from each seed point was set to 3000. The look-up table for orientation sampling matched to FA was computed using the Bingham distribution. Probabilistic local single- 
direction tractography was performed with the Camino software package (Cook et al., 2006).

Single-direction global (SDG)

Graph-based tractography as described by Iturria-Medina et al. (2007) was performed. The 3D neighborhood size was set to $5 \times 5 \times 5$ for each voxel to be connected to 98 nearest neighbors as edges in the tractography graph. This samples the fitted DT ellipsoid along 98 unique directions using the entire solid angle of $2 *$ pi/98 to compute edge weights. Connection's strength between two WMbound voxels is computed by multiplying the connection weights along the shortest path which connects them. Global single-direction graph tractography was performed with custom-written $\mathrm{C}++$ code.

Local multi-direction deterministic (MDD)

Deterministic multi-direction streamlines were obtained using a local co-linearity based approach (Hagmann et al., 2008 and Wedeen et al., 2008). That is, local direction finding consisted in following the local direction most colinear to the current fiber tangent. Nearest neighbor interpolation of the multi-direction field was performed. At every starting seed point, streamlines were initiated parallel to every local direction independently (i.e. maximally $27 \times 3$ streamlines per voxel). Deterministic local multi-direction tractographywas performed with custom-written $\mathrm{C}++$ code.

Local multi-direction probabilistic (MDP)

The employed algorithm uses orientations sampled from the FOD at each step and initializes a great number of streamlines per seed point in a way similar to the PICo algorithm. Per seed point, 3000 streamlines were initiated within a sphere whose center corresponds to the center of every WM boundary voxel and whose radius has been set to half the voxel size $(1.25 \mathrm{~mm})$. Probabilistic local multi-direction tractography was performed using the MRtrix package (Tournier et al., 2007). 
Multi-direction global (MDG)

When dealing with multiple directions extracted in every voxel, the concept of a tractography graph (Iturria-Medina et al., 2007) can be extended to the one of a multi-graph (Sotiropoulos et al., 2010). In this case, the center of every voxel is not just a single node, but a collection of $\mathrm{j}$ nodes, where $\mathrm{j}$ is the number of directions extracted in a specific voxel. Therefore, the weight of an edge connecting two neighboring voxels will not be a scalar anymore, but a j $\times$ m matrix, where $\mathrm{j}$ and $\mathrm{m}$ are the number of directions extracted in the two considered voxels. Note that although the graph-based algorithms used here (Iturria-Medina et al., 2007 and Sotiropoulos et al., 2010) do not account for edge length in the local edge weight calculation, this could be included in the algorithm (Zalesky, 2008). As for single-direction global tractography, the 3D neighborhood size was set to $5 \times 5 \times 5$ for each of the $\mathrm{j}$ direction voxel to be connected to $98 * \mathrm{j} * \mathrm{~m}$ nearest fibercomponent neighbors. This samples the fitted higher-order tensor along 98 unique directions using the entire solid angle of $2 *$ pi/98 to compute edge weights. Multi-graph tractography was implemented in customwritten $\mathrm{C}++$ code.

\section{Connectivity matrix weight computation and graph analysis}

To move from a very high resolution tractography result that connects all $\sim 30 \mathrm{k}$ voxels in the WMbound voxel set to the $4000 \times 4000$ binarized adjacency matrix, the following steps were followed. For the deterministic algorithms, any streamline connecting any voxel in one parcel to any voxel in another, connects the two parcels in the final adjacency matrix (c.f. Zalesky et al., 2010). For the probabilistic and global algorithms the threshold on percentile visitation counts or percentile graph weights was varied (between $80 \%$ and $99 \%$ ) to investigate the effect of probabilistic thresholding. Furthermore, the result from a probabilistic method is asymmetric (the count or weight for A seeded from B is not necessarily equal to the count or weight for $B$ seeded from A). Thus, any non-zero count or weight connecting any 
voxel in one parcel to any voxel in another in either of the two directions, connects the two parcels in the final symmetric adjacency matrix (c.f. Iturria-Medina et al., 2011). These binarization steps to construct the final $4000 \times 4000$ adjacency matrix are aimed at eliminating the effect of an arbitrary threshold, patch-area normalization or fiberlength normalization. For each binarization the edge density is computed asK/((N2 - N)/2) with $\mathrm{K}$ the number of supra-threshold edges and $\mathrm{N}$ the number of nodes, which here is always 4000 .

Computation of graph measures was performed using the Brain Connectivity Toolbox (Rubinov and Sporns, 2010). The small-worldness index is defined as the ratio of the normalized averaged clustering coefficient with the normalized path length of the considered graph (Watts and Strogatz, 1998). To normalize the average clustering coefficient and the path length of a network means to compare both measures with those obtained from a random network which is matched in terms of degree distribution. Given the size and the numbers of the graphs used in the present study, the approach proposed by Zalesky et al. (2010) was used, in which the average clustering coefficient and the path length of a random network was estimated using the analytical formula for an equivalent Erdös-Rényi random graph. A connectome is said to be small-world if the ratio of the two normalized indexes is higher than one, with the normalized clustering coefficient larger than one and the normalized path length close to one.

\section{Connectome dissection quality control}

We suggest an empirical approach to quality control the tractography results that uses connectome dissection. The connectome is partly dissected into a few large associative bundles which are known to exist. These are selected by specifying two cortical end ROIs (c.f. Catani and Thiebaut de Schotten, 2008 and Ffytche et al., 2010). The choice of tracts and end ROIs partly dissects the connectome adjacency matrix into a few submatrices, each of which corresponds to the selected bundle (Cammoun et al., 2012). If anatomical expertise is used to select the end 
ROIs carefully, one can now use the density (patch-to-patch connection count) of each of these submatrices as an empirical index of sensitivity. We define the tract specific density coefficient (TSDC) as:

$$
T S D C=\frac{K_{\left\{N_{i}\right\},\left\{N_{j}\right\}}}{\left|N_{i}\right|+\left|N_{j}\right|}
$$

where $\mathrm{N}_{\mathrm{i}}$ and $\mathrm{N}_{\mathrm{j}}$ are the sets of nodes (cortical patches) corresponding to the $\mathrm{i}$-th and the $\mathrm{j}$-th regions of interest, respectively, $|\mathrm{N}|$ is the number of nodes in a set and $\mathrm{K}\left\{\mathrm{N}_{\mathrm{i}}\right\},\left\{\mathrm{N}_{\mathrm{j}}\right\}$ is the count of non-zero entries of the submatrix of the whole brain adjacency matrix defined by the two node sets. The TSDC always ranges from 0 to 1 and quantifies the inferred strength of a known tract as expressed in the whole brain adjacency matrix. 0 signifies absolute absence of inferred connections (low sensitivity in case of a known tract). Since we normalize by $\left|\mathrm{N}_{\mathrm{i}}\right| \cdot\left|\mathrm{N}_{\mathrm{j}}\right|$, the maximum value of 1 would mean that every single patch in an ROI is connected to every single patch in the second ROI. This kind of complete mutual convergence/divergence is neurophysiologically not necessarily plausible in large associative cortico-cortical projections. This means that although the target for a known connection is a TSDC considerably higher than 0 , one would normally not want a TSDC that approaches 1. As a convention, we suggest here that a TSDC as a QC measure for sensitivity (a sensitivity TSDC, to be contrasted with a specificity TSDC, see below) should range between 0.1 and 0.5 for a cortical parcellation with 4000 nodes. Although the normalization does not change the result of any comparison between two different tractography methods for the same tract, it crucially enables absolute interpretation of TSDCs and comparisons between different tracts. Conceivably, it could be used to quality control entire connectome matrices by requiring TSDCs in a certain range for a considerable number of known tracts, which together span a large fraction of the adjacency matrix. 
Here there are three important issues. First, as its name implies, a TSDC is very specific to a tract and the accurate expert identification of its end ROIs. Comparing algorithms with each other on the basis of TSDCs will require looking at a couple of tracts. Here we have chosen three (TSDCCC, TSDC SLFII and TSDCILF) that together cover an important subset of the challenges that tractography algorithms face. The ROIs for selecting these three bundles were derived from the literature (Catani and Thiebaut de Schotten, 2008,Ffytche et al., 2010 and Wakana et al., 2004) and manually selected on the reconstructed WMbound surface. After a first manual selection, the ROIs are then constrained by the actual parcellation used for the definition of the nodes of the connectome, so that the refined ROI only contains those cortical patches which are entirely comprised in the first selection. For the CC we selected a triangular patch whose vertices are given by the anterior part of the superior frontal gyrus, the dorsal part of the precentral gyrus and theinferior frontal gyrus. For the SLFII we selected patches belonging to the dorsolateral prefrontal cortex and the inferior parietal cortex as outlined in Ffytche et al. (2010) and Thiebaut de Schotten et al. (2011). Note that there may be hemispheric differences (rightward asymmetry) in the SLF II (Ramayya et al., 2010); much like those that have been found repeatedly for the arcuate fasciculus (leftward asymmetry). Here, we have only selected the ROIs lying on the left hemisphere. Finally, for the ILF we selected patches lying on the anteriormost part of the temporal lobe (temporal pole) and on the posteriormost part of the occipital lobeas outlined in Catani and Thiebaut de Schotten (2008) and Wakana et al. (2004). Second, the behavior of TSDC is influenced by the choice of the number of nodes that span the cortex obtained by the cortical parcellation. High values approaching 1 are more easily obtained for smaller number of nodes and coarser cortical parcellation since tract specific submatrices of the connectome adjacency matrix become denser (Cammoun et al., 2012). Third, TSDCs for a set of tracts as an evaluation of sensitivity must be balanced by also evaluating specificity. This must be done in at least two ways. We first verify that 
the TSDC for two ROIs which are known not to be connected (specificity TSDC) is small. This requires identifying a set of pairs of non-connected ROIs. Here, for illustration, we chose a set of subregions of the ROIs used to track the corpus callosum, which causes them to be distancematched with the corpus callosum tracts. Crucially, we selected the anteriormost part of the superior frontal gyrus in the left CC ROI and the much more posterior precentral gyrus in the right CC ROI. Although heterotopic connections through the CC are known to exist in the frontal cortex ( Jarbo et al., 2012), and the precentral gyrus might be connected to the contra-lateral posteriormost superior frontal gyrus, we would expect a-priori that there are no connections between these two ROIs. Thus, as a first quantitative measure of specificity we require the TSDC computed for these ROIs to be very small. However, this procedure will potentially miss many false positives because even if tracts connect two true-positive ROIs with a high TSDC, the tract paths themselves might take an erroneous course between them. Therefore, secondly we evaluate sensitivity TSDCs for known connections in conjunction with careful inspection of tractography path results to identify erroneous tracking results.

\section{Results}

Fig. 1 shows the edge density of the final adjacency matrices for all methods. The results are very consistent over subjects and parcellations (the standard error computed over subjects and parcellations was maximally 0.001 for local approaches and 0.003 for global approaches across all parameter combinations). Density always decreases with increasing probabilistic threshold: as more of the probabilistic tractography results fall below threshold, final graph sparseness increases. In a similar way, both a change in FA threshold from 0.2 to 0.1 and a change in angle threshold from $30^{\circ}$ to $90^{\circ}$ tend to roughly double the connection density. Generally, as can be expected, more liberal thresholds (lower probabilistic, FA or minimum curvature thresholds) 
lead to higher global connection density. Less intuitive, but evident in our analyses, global methods tend to have up to twice the connection density of local methods at the same thresholds. The effect of probabilistic versus deterministic approaches is most clearly seen in Fig. 1 at the $30^{\circ}$ angle threshold. As discussed above, one can take a deterministic interpretation of a global tractography result by considering only the highest percentile results. Interestingly, this 'deterministic limit of probabilistic results' is also found experimentally in the local multi-direction results at a $30^{\circ}$ angle threshold. This is interesting given that (in contradistinction with the global methods) this represents a convergence in results in two separate local tractography approaches.
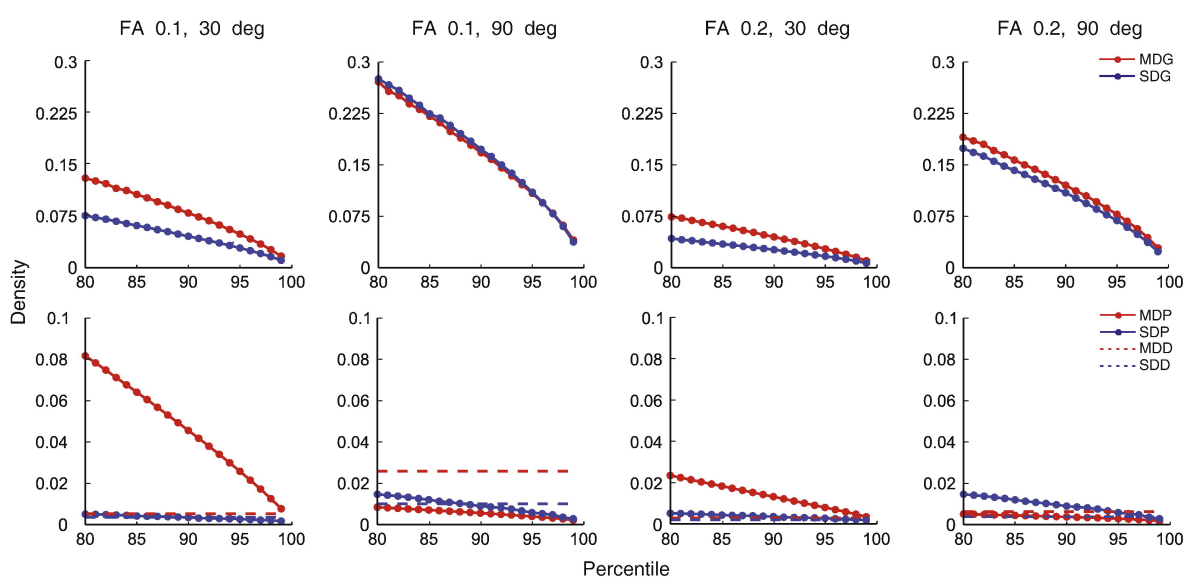

Figure 1: Edge-density of the final adjacency matrices averaged over two subjects. Global approaches in the upper row, local approaches in the lower row. The columns represent different combinations of anisotropy and angle thresholds. On the horizontal axis different probabilistic thresholds are plotted for probabilistic methods; deterministic methods are plotted as a single straight line. Single-direction methods are plotted in blue, multiple-direction methods in red. Note the different scales on the y-axis.

Local probabilistic methods at very liberal thresholds, especially a $90^{\circ}$ angle threshold in absence of a colinearity constraint based on prior identification of main diffusion directions can lead to degeneration of algorithm performance and pathological results. This manifests itself 
in the reversal of some of the patterns above, such as a higher density for the single direction method. This degeneracy is especially applicable to local probabilistic multi-direction methods, presumably because of the greater noise sensitivity of these more complex methods in combination with very permissive probabilistic direction sampling.

Since topological graph indices can be strongly dependent on the edge-density of the network (van Wijk et al., 2010), we plot all subsequent results as a function of edge-density. Density generally decreases: i) with increasing probabilistic threshold, ii) when going from probabilistic to deterministic tractography, iii) with increasing FA and decreasing angle thresholds, iv) when moving from global to local tractography, and $\mathrm{v}$ ) when moving from multi direction to single direction models. It is important to only compare indices between density matched graphs in order to assess the true effects of tractography algorithms and parameters on the topology of whole brain adjacency matrices independent of their simple effects on global density as reported above. However, because adjusting the probabilistic threshold generally has an effect both on density and possibly on topology, we have included corresponding figures plotted as a function of percentile threshold in the supplementary material.

Fig. 2 shows deterministic streamline length histograms for single and multi-direction methods at different angle thresholds. Note that only streamlines connecting two WMbound voxels are considered and that many more streamlines are started for MDD (in all FOD directions at each seed point) than for SDD (in one direction at each seed point). The first significant aspect is considerable short-length $(<20 \mathrm{~mm})$ streamline abundance, especially for the multi direction method. Although numerous, streamlines of this short length are unlikely to contribute much to the final adjacency matrix, since they will often connect a node-ROI with itself, which is neglected. In other words, one expects longer streamlines to contribute most to adjacency matrix density. Indeed, a larger number of long (between 40 and $160 \mathrm{~mm}$ ) 
streamlines in these histograms correspond to a higher density in Fig. 1. This is seen prominently in MDD having a greater density than SDD at the same thresholds. The short streamline abundance is considerably tempered by a more liberal angle threshold $\left(90^{\circ}\right)$, leading to less early termination and hence a greater number of longer streamlines.

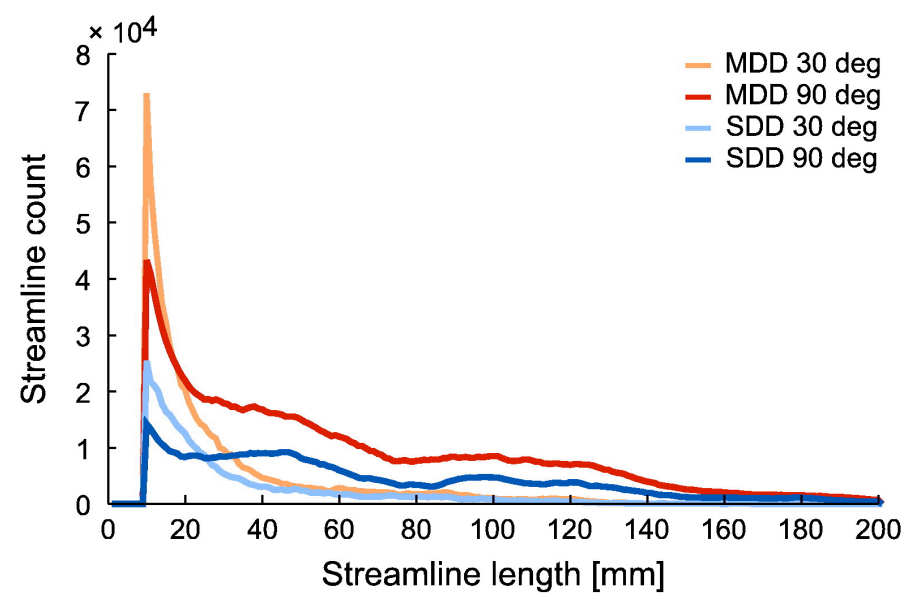

Figure 2: Length histograms (1 $\mathrm{mm}$ bins) for deterministic streamline tractography methods at FA threshold 0.1 averaged over 2 subjects. Only streamlines connecting WMbound voxels are considered. Note that based streamlines shorter than $10 \mathrm{~mm}$ and longer than $200 \mathrm{~mm}$ are excluded.

Fig. 3 shows the small-worldness index of the final adjacency matrices for all methods. First, it is observed that small-worldness (a coefficient larger than one; Watts and Strogatz, 1998) is a very robust phenomenon in human structural networks obtained with DWI tractography. It is observed over all tractography methods and parameter values and its value is very consistent over subjects and parcellations (the standard error computed over subjects and parcellations was maximally 3.621 for local approaches and 0.3 for global approaches across all parameter combinations). This agrees with and extends the finding of reproducibility of network indices over scanning session and individuals for local deterministic methods (Bassett et al., 2011b). Considerable effects of tractography algorithm on 
small-worldness can be observed even when adjacency matrices are density matched. At FA threshold 0.2 and angle threshold $30^{\circ}$, MDD and SDD have coefficients 70\% larger than for density matched SDP. At the same FA and angle thresholds and a density of 0.005 MDP has a coefficient $26 \%$ larger than for density matched SDP. Global versus local differences also are found to be up to $30 \%$, for instance at FA 0.2 and angle 30 and a density of 0.01, MDG and MDP have small-worldness coefficients of, respectively 21.83 and 28.45 . Contrary to these effects of algorithm choice, small-worldness effect to be relatively insensitive to FA and angle thresholds.

The small-worldness coefficient results are best illustrated further by separately inspecting the two components it consists of: normalized path length and normalized average clustering coefficient, reported in Fig. 4 and Fig. 5, respectively. Once again, the values for normalized path length and normalized average clustering coefficients are very consistent over subjects and parcellations (with a standard error over subjects and parcellations of maximally 0.093 for local approaches and 0.008 for global approaches for normalized path length, and maximally 1.431 for local approaches and 0.885 for global approaches for normalized average clustering coefficients). A larger degree of small-worldness can be caused by a smaller normalized path length, a larger normalized average clustering coefficient or both. For small world networks the normalized path length should be close to one, which is true for most methods and parameter sets, except for the SDP and the MDP for the $90^{\circ}$ angle threshold or for low densities (high probabilistic thresholds). The small-worldness difference of MDD and SDD with density matched SDP is seen to be explained by a normalized path-length for MDD and SDD that is 70-80\% lower than that for SDP. Similarly, there is a $23 \%$ difference between SDP and MDP at FA 0.2 and angle $30^{\circ}$, with normalized path length for SDP larger than for MDP which explains their difference in small-worldness reported above. 
Finally, the local/global effect in small-worldness can also be traced back to path-length, with 2.3 for MDG and 1.9 for MDP.
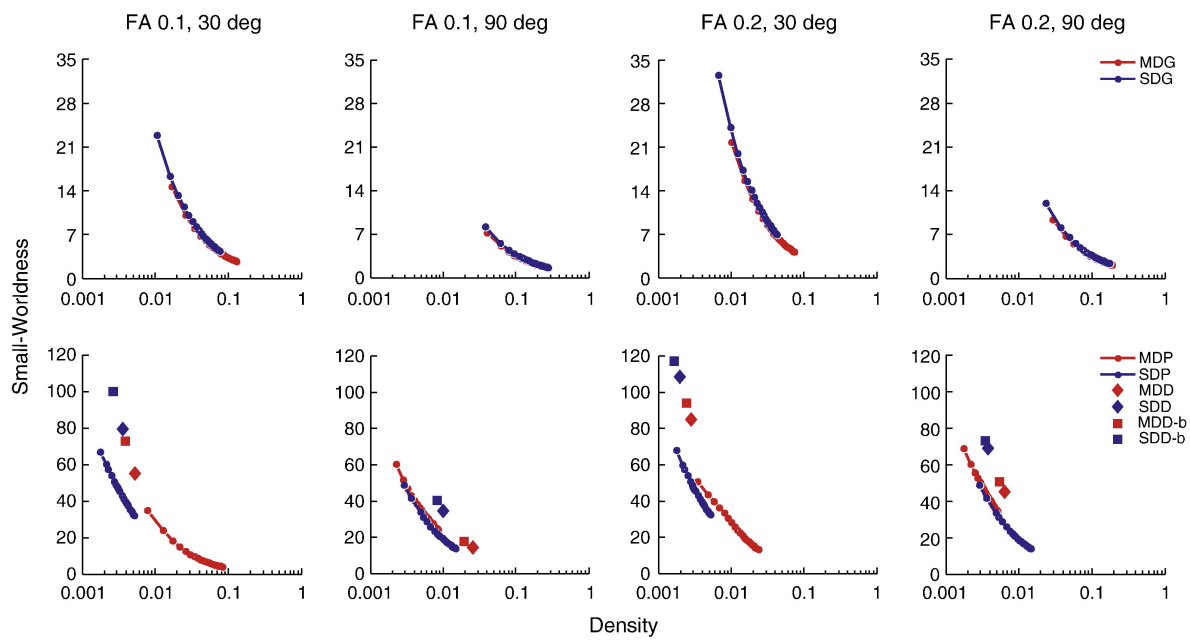

Figure 3: Small-worldness plotted as a function of density of the final adjacency matrices averaged over two subjects. Columns, rows and colors are organized as in Fig. 1. Deterministic methods seeded from the entire WM are plotted as diamonds; deterministic methods seeded only from the WMbound are identified by a ' $-b$ ' at the end and plotted as squares. Note the different scales on the y-axis.
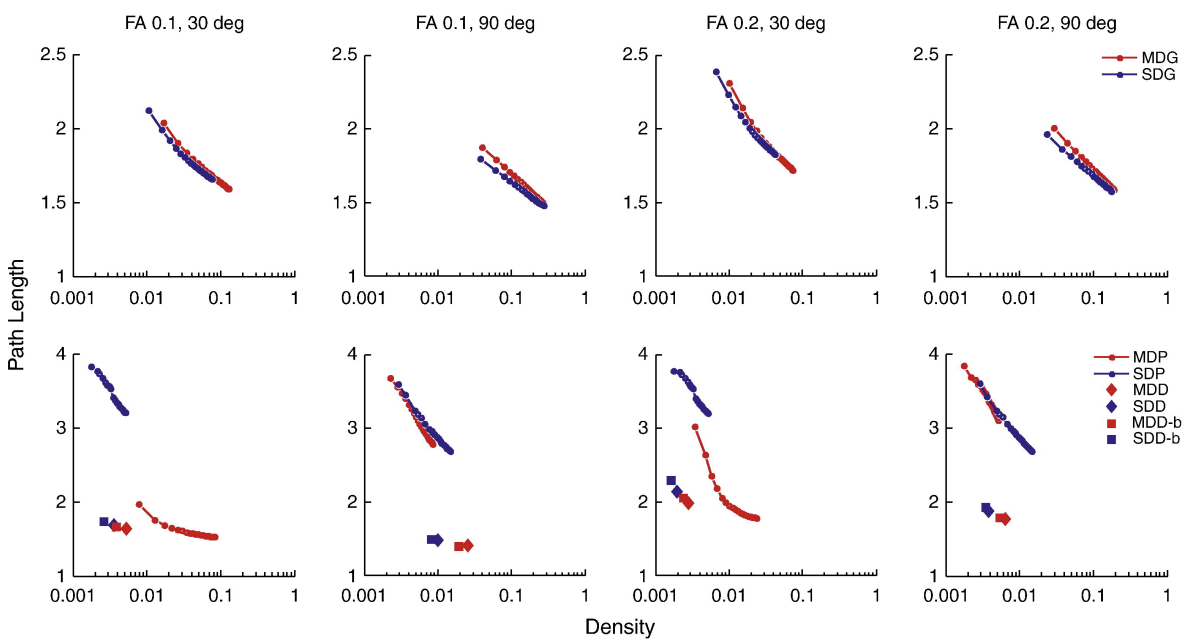

Figure 4: Characteristic path length averaged over 2 subjects, organized as in Fig. 3. 

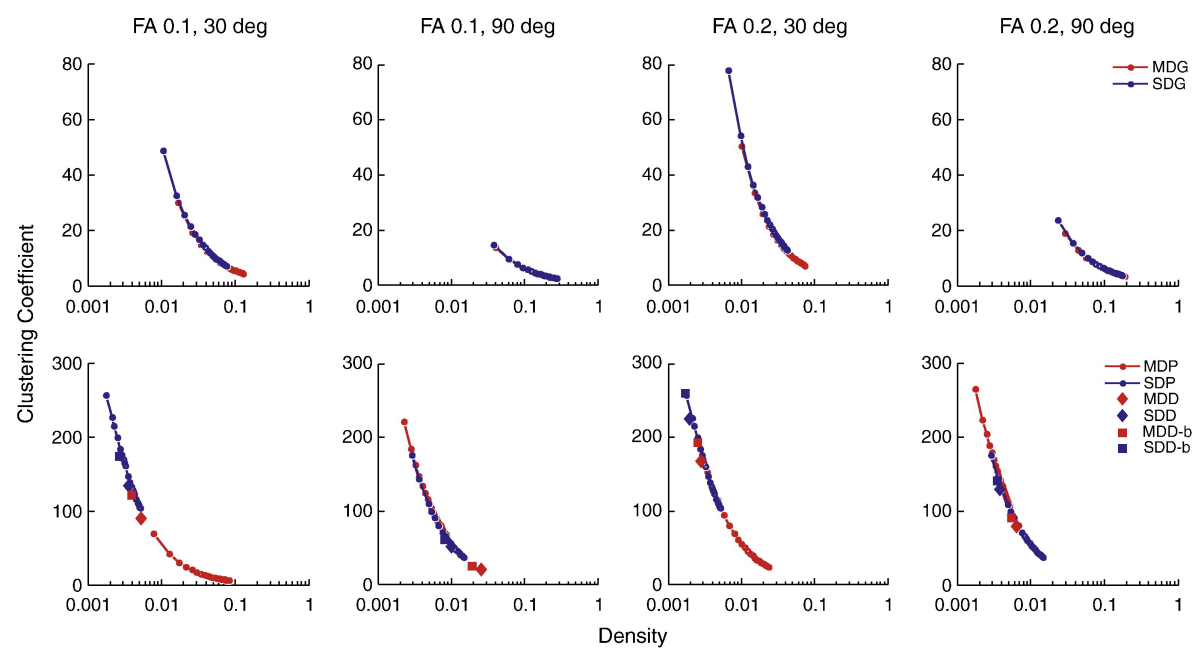

Figure 5: Average clustering coefficient averaged over two subjects, organized as in Fig. 3.

Thus, the small-worldness results are largely contained within the effect on normalized path-length, as is indeed supported by Fig. 5 showing a relatively little effect of algorithm choice on clustering coefficient. However, in the low density ranges, where deterministic and probabilistic local approaches can be compared in terms of density and the curve is steeper, differences are still up to $10 \%$. Interestingly, the absence of effect of FA and angle threshold on smallworldness is not explained by the absence of effect on its constituents. Instead, the effect of threshold parameters on both normalized path length and clustering coefficient are large but equal, which leads them to be divided out in the small-worldness coefficient.

Finally, global network efficiency is another graph measure that is often used to quantify the overall shortest path length distribution. It is inversely related to the characteristic path length (Fig. 4). Indeed, Fig. 6 (standard error computed over subjects and parcellations was maximally 0.002 for local approaches and 0.003 for global approaches across all parameter combinations) shows global approaches 
to be more efficient than local, multi direction to be more efficient than single direction and global network efficiency to increase with increasing density.
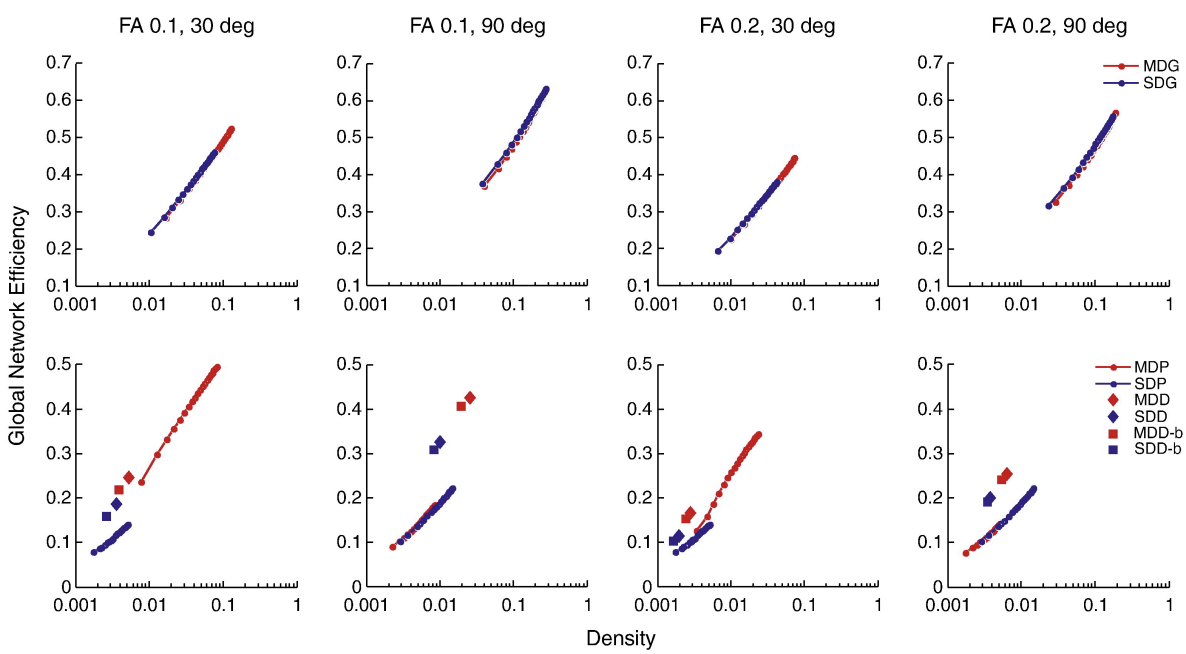

Figure 6: Global network efficiency averaged over two subjects, organized as in Fig. 3.

For the connectome dissection quality control (QC) we chose to select the second branch of the superior longitudinal fasciculus (SLFII), the inferior longitudinal fasciculus (ILF), both in the left hemisphere, and part of the transcallosal projections (CC) for subject 2 as shown in Fig. 7. Fig. 8 plots TSDCCC, TSDCsLfil, TSDCilf and TSDCFp for four different tractography algorithms and over a range of percentile thresholds for probabilistic algorithms. Here TSDCFP is the TSDC for the selected false positive connection between the anteriormost part of the superior frontal gyrus in the left CC ROI and the much more posterior precentral gyrus in the right CC ROI. Note that, in what follows, the probabilistic threshold is always selected on the basis of the whole brain tractography results. Hence, these thresholds are not necessarily equivalent to selecting the nth percentile of paths connecting just the selected ROIs, but they are equal to those applied for the whole brain adjacency matrices. Focusing first on the sensitivity TSDCs, at very 
low percentile thresholds for CC and SLFII graph-based global algorithms have TSDCs close to 1, whereas TSDC ILF for the selected example is an order of magnitude lower for reasons we discuss below. TSDCs for deterministic algorithms range between 0.007 and 0.11 for the selected tracts, with TSDC for MDD being always 2 to 10 times higher than that for SDD for the same tract. Finally, TSDCs for graph based algorithms approach those of deterministic algorithms only for very high thresholds (99 th percentile; again we discuss the ILF below).

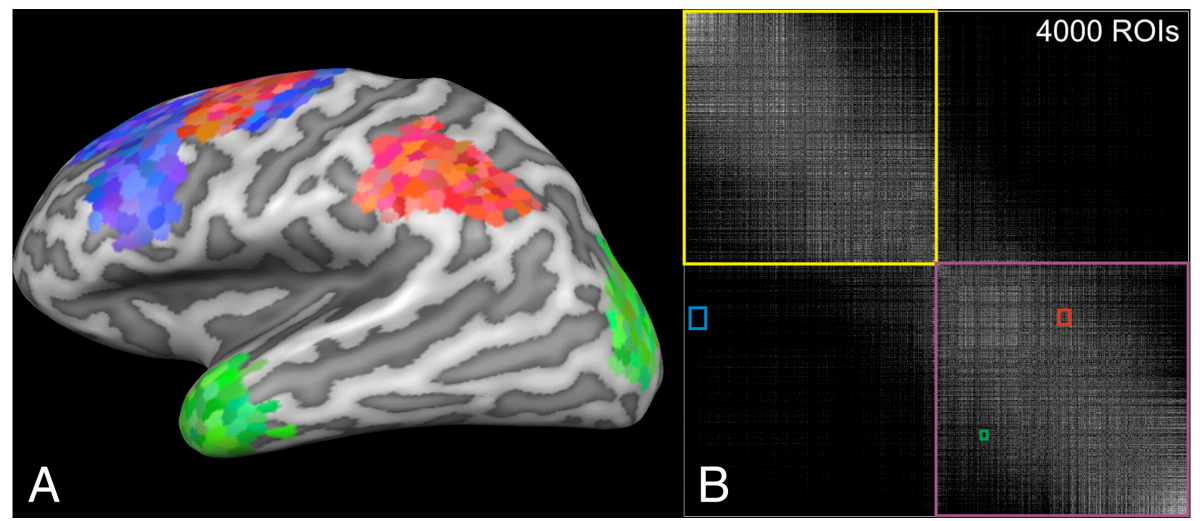

Figure 7: An illustration of the suggested connectome dissection QC approach. A) The selected pairs of cortical end ROIs: SLF II (in red), ILF (in green) and part of the transcallosal connections (in blue) shown on an inflated cortical surface representation. Different shades of the same colors are used to highlight the individual cortex patches which form the nodes of the adjacency matrix. B) The corresponding selected submatrices in the connectome adjacency matrix. Yellow and purple squares represent the right and the left hemispheres, respectively.

Therefore, we will focus on comparing the tractography results mostly at the intersections of the global and local TSDC curves below. Focusing next on the specificity (false positives) TSDCFP, pleasingly it shows false positive TSDC scores to be much lower than sensitivity TSDCs. As one would expect for stochastic algorithms when thresholds are low enough, significant false positives start to arise. However it can be seen that for probabilistic thresholds above the $90^{\text {th }}$ percentile false positive TSDC falls below 0.01. Interestingly, this offers a partial 
solution to choosing a good operating range of probabilistic thresholds, namely that range which simultaneously keeps sensitivity TSDCs between 0.1 and 0.5 and specificity TSDCs below 0.01 . Here this would amount to a threshold between the $91^{\text {st }}$ and the $98^{\text {th }}$ percentiles. Sensitivity TSDCs which fall below 0.1 (as for the ILF here) point to infidelities in tractography results, which illustrate its role as a quality control criterion as discussed below.
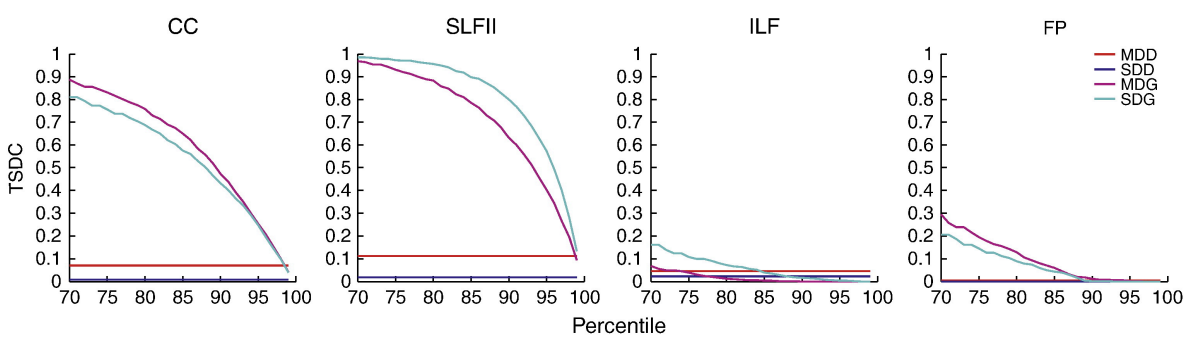

Figure 8: Plots of TSDCCC, TSDCSLFI, TSDC ILF and TSDCFP for four different tractography algorithms at threshold FA 0.1 and $90^{\circ}$. Global algorithms are plotted at 30 different percentiles.

Specificity TSDCs will potentially miss false positives because even if tracts connect two true-positive ROIs with a high TSDC, the tract path between themselves might take an erroneous course. Therefore, we continue to evaluate false positives by carefully examining tractography paths for sensitivity TSDCs. We begin our evaluation with the wellknown and often used example of the inter-hemispheric projections through the body of the corpus callosum. It is known that the transcallosal projections connect a large inferior-superior extent of the lateral frontal cortex. This is reflected in our selection of cortical ROIs for the $\mathrm{CC}$ in Fig. 7 which spans the lateral frontal cortical surface (from the lateral superior frontal gyrus to the operculum) for a few centimeters anteriorly to the central sulcus on both hemispheres. These trans-callosal projections pass through the body of the corpus callosum (CC) and cross with several other major fiber tracts. Among these are the superior longitudinal fasciculus (SLF) and the corona radiata (CR), both of which 
cross and intersect with each other and with transcallosal fibers in the centrum semi-ovale. Thus, the ability of a tractography algorithm to connect the entire inferior-superior extent of the lateral frontal cortex through a three-way crossing is reflected in the TSDCcc. Fig. 8 shows that the TSDCcc is greater for multiple direction approaches than for single direction and greater for global methods than for local methods for all probabilistic thresholds (except the $99^{\text {th }}$ percentile compared to MDD).

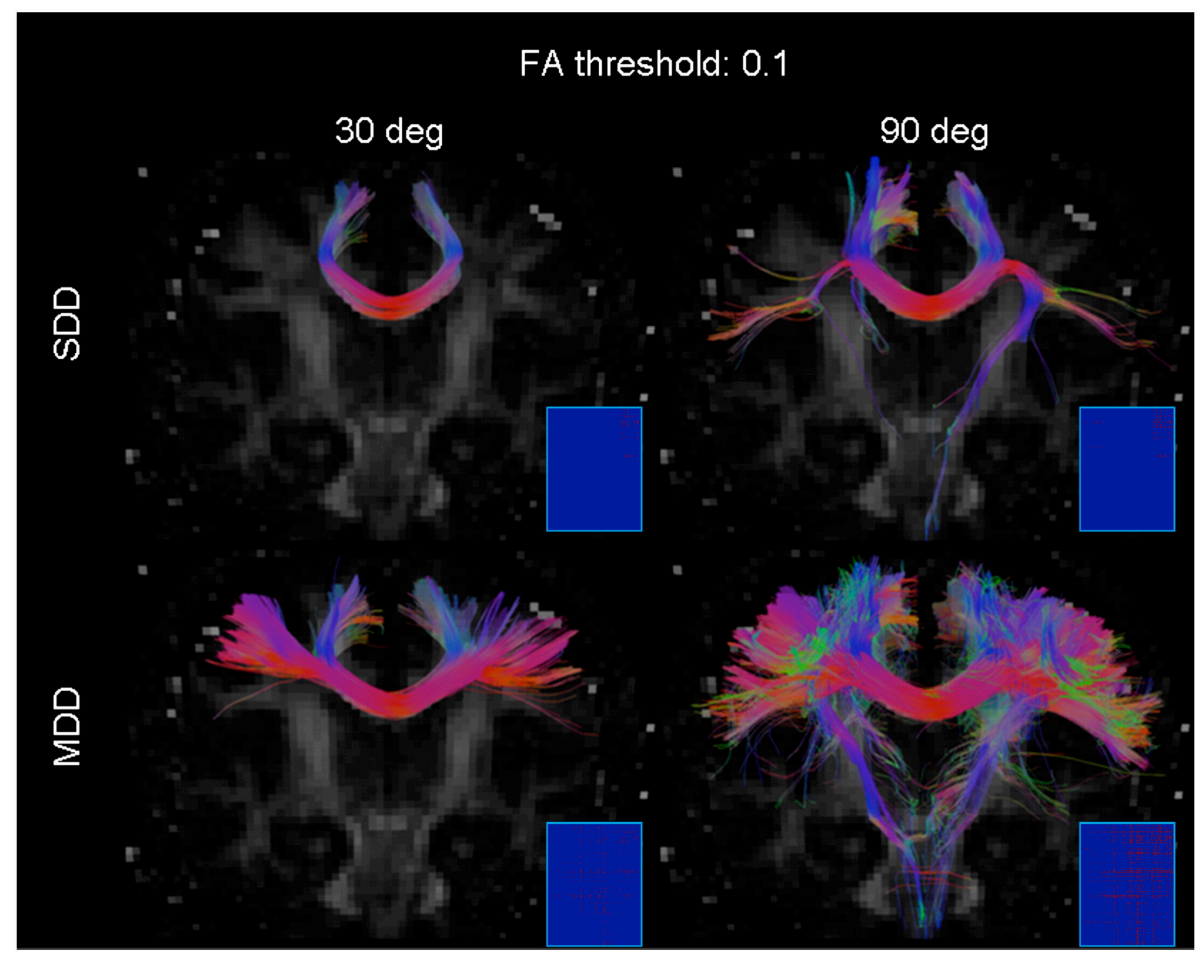

Figure 9: Direction color-coded streamlines that connect the two CC ROIs on the left and right lateral frontal cortices in subject 2 for SDD (top row) and MDD (bottom row) at FA threshold 0.1 and two different angle thresholds. The corresponding submatrices obtained from the adjacency matrix (see Fig. 7) are shown for every combination. 


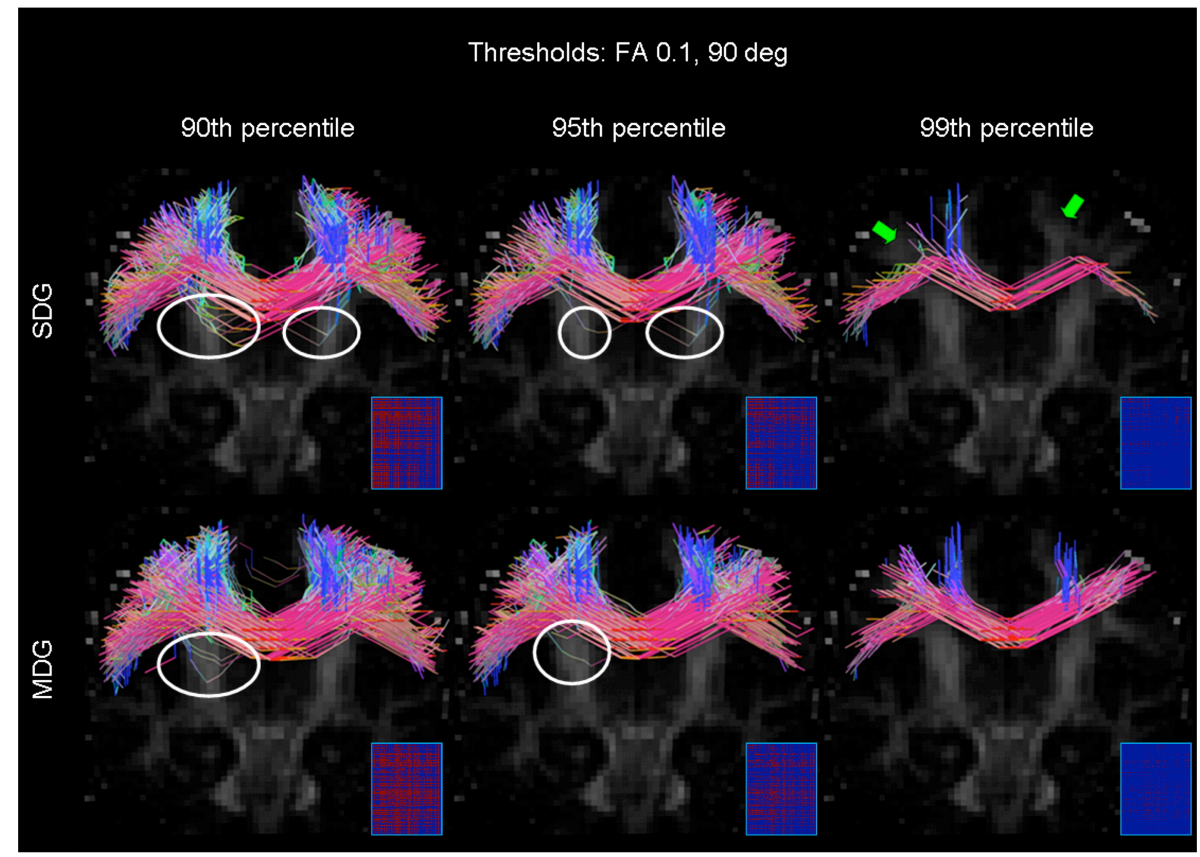

Figure 10: Direction color-coded reconstructed fiber paths that connect the two CC ROIs for SDG (top) and MDG (bottom) in subject 2. From left to right, probabilistic thresholds are set to the $90^{\text {th }}, 95^{\text {th }}$ and $99^{\text {th }}$ percentiles. All paths are reconstructed using FA 0.1 and $90^{\circ}$ as thresholds. White circles and green arrows represent false positives and false negatives, respectively. The corresponding submatrices obtained from the adjacency matrix (see Fig. 7) are shown for every combination.

To illustrate the difference in the actual streamlines or tractography paths that underlie these differences in TSDC (and global statistics of the adjacency matrices in Fig. 1, Fig. 2, Fig. 3, Fig. 4, Fig. 5 and Fig. 6), Fig. 9 and Fig. 10 show the corresponding local and global tractography path results. Fig. 9 shows deterministic streamlines for SDD and MDD at a liberal FA threshold and at two angle thresholds (the right-hand 90 degree panels correspond to the TSDCcc reported in Fig. 8). The absolute number of streamlines and average lengths for each of the four cases are listed in Table 2 (compare to Fig. 2) and the connected patches on the cortex are reported in Supplementary Fig. S5. Fig. 9 shows two effects which can impact global statistics. First, MDD is more successful in identifying the lateral transcallosal projections (c.f. 
Tuch et al., 2003 and Wedeen et al., 2008), which is confirmed by a higher TSDCcc in Fig. 8. Even at very liberal anisotropy and angle thresholds SDD only identifies a few lateral callosal projections, some of which track wrongly into the SLF or CR along their way. That is, there is a large amount of false negatives in the SDD results, even at liberal thresholds. Second, the MDD results at two different angle thresholds show a classical sensitivity/specificity trade-off. A few of the inferior lateral trancallosal projections which are missed at the $30^{\circ}$ threshold are correctly reconstructed at a $90^{\circ}$ threshold. However, this is at the cost of a large number of false positive streamlines that, again, connect both lateral surfaces to SLF or CR pathways. This sensitivity/specificity tradeoff for the transcallosal projection is improved further in the corresponding SDG and MDG results shown in Fig. 10 with the corresponding connected cortical patches reported in Supplementary Fig. S6. Here, results are shown for the same liberal thresholds on FA and angle as Fig. 8 and in the right side of Fig. 9, now at three different probabilistic thresholds. Note that, because of virtually equal density adjacency matrices at any threshold for these cases (see Fig. 1, upper row, second panel), we are justified in quantitatively comparing the SDG and MDG connections for the same percentiles (van Wijk et al., 2010). It can be seen that at $90^{\text {th }}$ and $95^{\text {th }}$ percentile thresholds most of the expected transcallosal inter-hemispheric projections are correctly identified with very few false positives with both methods. One can observe a greater tendency in SDG for false negatives at the green arrows compared to MDG in the $99^{\text {th }}$ percentile and slightly more false positives for SDG in the white circle in the $95^{\text {th }}$ percentile. In summary, the greater sensitivity and more complete inference of the transcallosal projections by MDG quantified by the TSDCcc is achieved without a compromise in false negatives as observed in the tractography paths and in the specificity TSDC in the $95^{\text {th }}$ and $99^{\text {th }}$ percentiles.

To assess the effect on connection topology in more spatial detail, Fig. 11 shows the spatial distribution of degrees for nodes on the 
cortical surface for both MDG and SDG for the same thresholds as Fig. 10. The effect of probabilistic threshold is to sharply decrease the absolute number of edges for each node and to focus the remaining edges more on the most highly connecting hubs. Spatial degree distributions can also be seen to be more focused for SDG than for MDG. Specifically, connection hubs identified by both approaches in the same location (for instance the lateral inferior frontal hub) are more spatially confined and have a higher absolute degree for SDG. However, MDG defines some hubs, such as the lateral superior parietal area, that are much less clear in the SDG.

\begin{tabular}{ccc}
\hline $\begin{array}{c}\text { Tractography } \\
\text { method }\end{array}$ & $\begin{array}{c}\text { Number of } \\
\text { streamlines }\end{array}$ & $\begin{array}{c}\text { Average streamline length } \\
{[\mathbf{m m}]}\end{array}$ \\
\hline SDD, $30 \mathrm{deg}$ & 3325 & 108.26 \\
SDD, $90 \mathrm{deg}$ & 11981 & 113.70 \\
MDD, 30 deg & 10703 & 92.03 \\
MDD, 90 deg & 49237 & 118.23 \\
\hline
\end{tabular}

Table 2: The absolute number of streamlines and average streamline lengths for each of the tractography results in Fig. 7.

Next, we look at the second branch of the superior longitudinal fasciculus (SLFII), which is a slightly shorter associative tract characterized by a high curvature and fewer tracts crossing it. Again Fig. 8 shows TSDCsLFI to be larger for global tractography methods than for local approaches except for the $99^{\text {th }}$ percentile compared to MDD. However, although MDD performs better than SDD, the TSDCsLfi for SDG is larger than that for MDG for all probabilistic thresholds. Fig. 12 shows the corresponding tractography paths, at the 99th percentile for SDG and MDG, corresponding to the intersection of local and global TSDCsLrI. It can be seen that at these high probabilistic thresholds the global algorithms preferentially connect the ROI patches 
that are spatially closest, a tendency that reduces with lower probabilistic percentiles (not shown for SLFII), which is also seen in the corresponding connected cortical patches reported in Supplementary Fig. S7. This can also be seen by a concentration of density in the corner of their adjacency submatrix. Instead patch connections for the local deterministic approaches, especially multi direction, are much more spatially spread over the ROIs. MDD is seen to detect more separated branches of the SLFII than SDD, SDG or MDG, which it is generally considered to have (ffytche et al., 2010 and Thiebaut de Schotten et al., 2011), and to have a greater spread of connections in the most posterior regions. Furthermore, the superior performance of SDG and MDD in terms of the higher TSDCSLFI seems to be combined with a low tendency for false positives. Finally, MDG is seen to be more sensitive to noise and even more constrained in the length of the tract than SDG in this non-crossing tract.

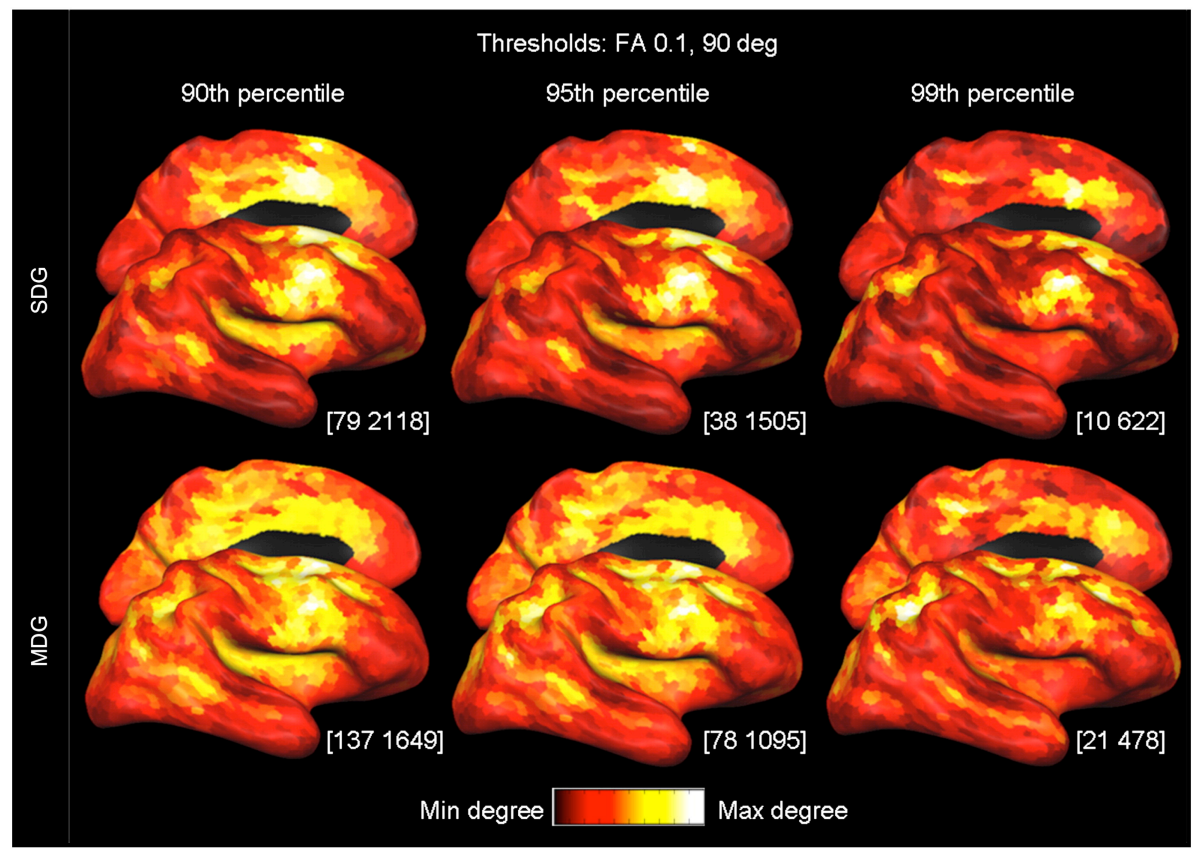

Figure 11: Degree distribution plotted on the inflated WM/GM boundary surface for subject 2. Top row: SDG, bottom row: MDG. Columns represent the probabilistic 
thresholding of the connectivity matrix for the $90^{\text {th }}, 95^{\text {th }}$ and $99^{\text {th }}$ percentiles. Between brackets are the min-max degree intervals for each combination.

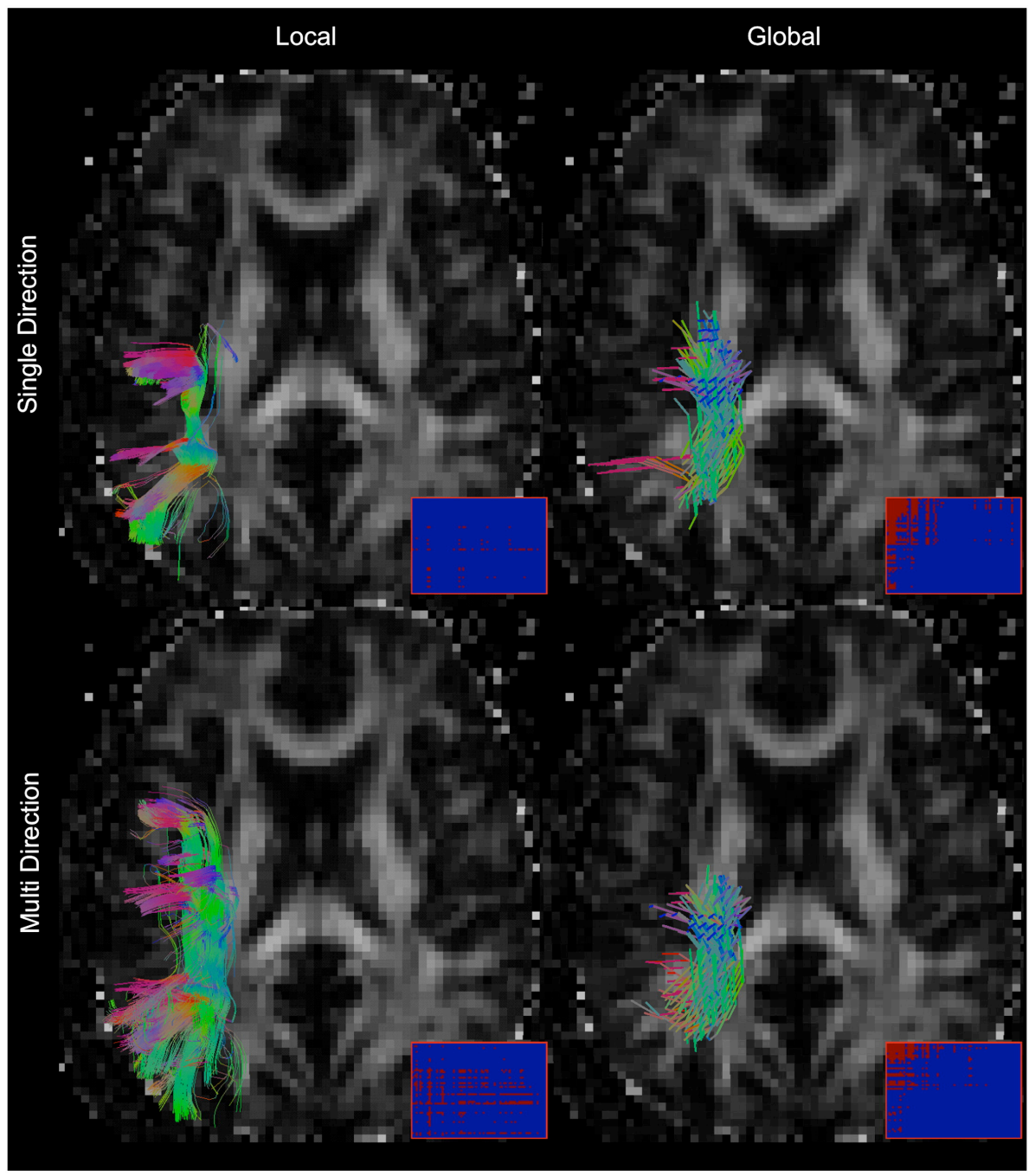

Figure 12: Comparison between reconstructed SLFII fiber paths in subject 2 using two different tractography approaches and the same thresholding parameters ( $\mathrm{FA}=0.1$, angle threshold $90^{\circ}$ ). Columns represent the type of tractography approach (left: local deterministic, right: global), rows represent the intra-voxel diffusion model employed (top: single direction DTI, bottom: multi direction CSD). Probabilistic thresholds for global approaches are obtained from Fig. 8 and are set to the $99^{\text {th }}$ percentile. All paths are reconstructed using FA 0.1 and $90^{\circ}$ as thresholds. The corresponding submatrices obtained from the thresholded adjacency matrix are shown for every combination. 
Finally we have selected the left ILF of subject 2 to show aberrant behavior that can occur even in data with good SNR when identification of local direction is imperfect. The ILF connects the anterior part of the temporal lobe to the occipital lobe. It is characterized by a very straight course along the temporal lobe and it is joined in its path by several other fibers, such as the inferior fronto-occipital fasciculus (IFO). In particular the arcuate fasciculus (AF) curves down from its course from the perisylvian cortex of the frontal and parietal lobes and converges to join the direction of the ILF (Catani and Thiebaut de Schotten, 2008). This convergence of directions of two major tracts within the imaging voxels creates particularly difficult local direction identification problem. This is because crossing fibers are harder to identify for multiple direction models as the different directions become more colinear. As can be seen in Fig. 13 with the corresponding connected cortical patches reported in Supplementary Fig. S8, the ILF is well identified by all methods. However, Fig. 8 shows TSDC ILF to be two to ten times lower than that for the other two tracts for all methods except for SDD. Furthermore, the TSDC ILF for SDG and MDG shows a much steeper decline with an increasing probabilistic threshold. More careful inspection of Fig. 13 shows the reason to be a relatively long tract in conjunction with imperfect local direction segregation highlighted by the zoomed inset. This shows MDD to take unrealistically nearly $90^{\circ}$ turns which seem to result from jumping out of the ILF into the AF and then converging back into the ILF again. MDG in contrast avoids tracing through this region altogether. This is because MDG's global shortest path finding mechanism penalizes such strong local curvature for the entire tract, which for the relatively long ILF is already penalized for its length in global probabilistic approaches. 


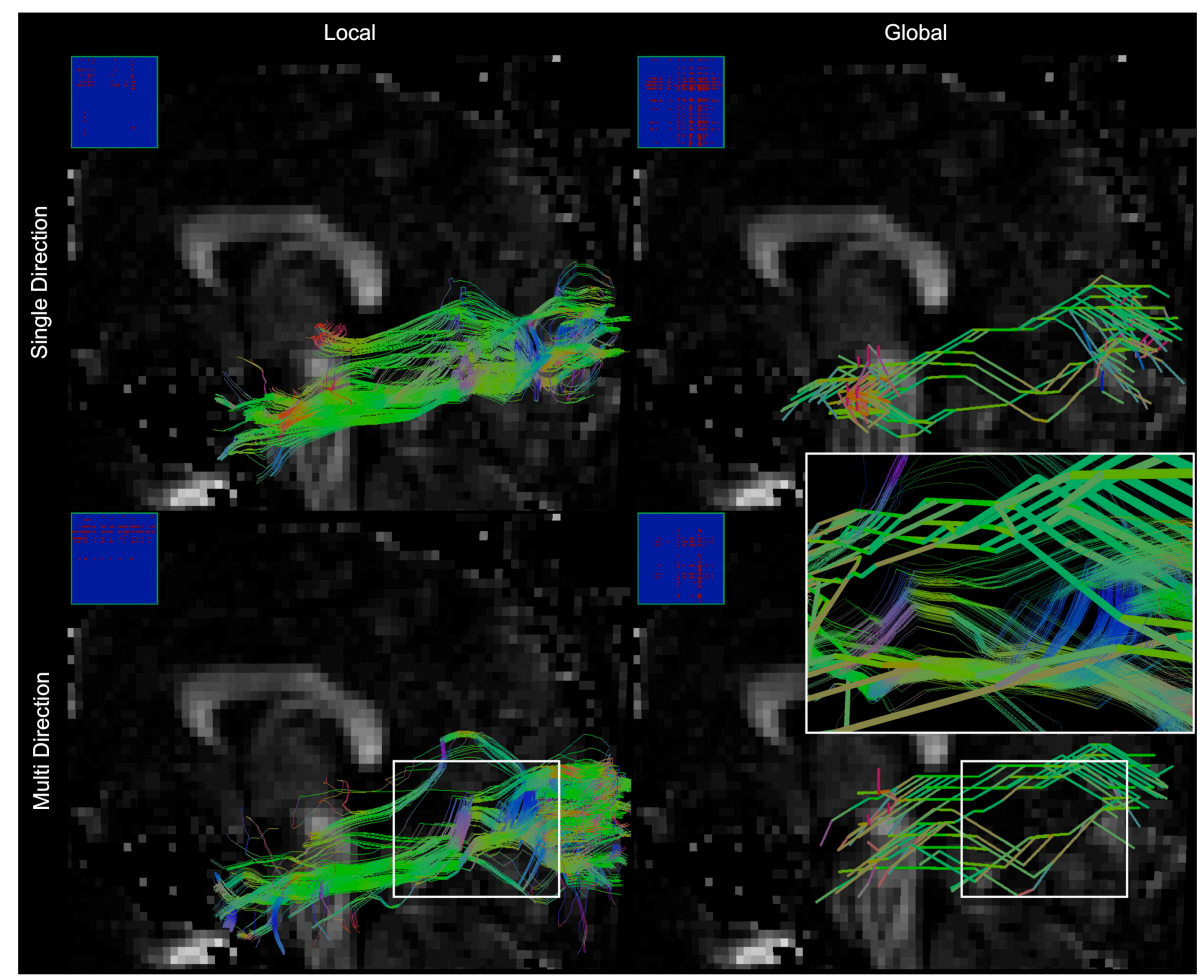

Figure 13: Comparison between reconstructed ILF fiber paths in subject 2 using two different tractography approaches and the same thresholding parameters $(\mathrm{FA}=0.1$, angle threshold $90^{\circ}$ ). Columns represent the type of tractography approach (left: local deterministic, right: global), rows represent the intra-voxel diffusion model employed (top: single direction DTI, bottom: multi direction CSD). Probabilistic thresholds for global approaches are obtained from Fig. 8 and are set to the $74^{\text {th }}$ percentile. All paths are reconstructed using FA 0.1 and $90^{\circ}$ as thresholds. The corresponding submatrices obtained from the thresholded adjacency matrix are shown for every combination. The zoomed inset shows both the MDD streamlines as thin lines and the MDG fiber paths as thick lines.

\section{Discussion}

We have investigated how different tractography algorithms can influence the estimation of several network indices that characterize the human structural connectome, including small-worldness, average clustering coefficient, characteristic path length, global network efficiency and high density nodes. We show that the choice of the general class of tractography algorithm in combination with the 
thresholds on FA, angle and probabilistic percentiles can dramatically influence these network indices. This is true in many cases even when global density differences are controlled for, showing that this marks actual differences in network topology.

It is important to note that the findings of small-worldness, high global network efficiency and high density hubs per se, characteristic for the human brain (Hagmann et al., 2008), are very robust over all investigated methods and parameters. These findings are also very consistent over two subjects and over different random surface parcellations for any of the tested combinations. It is rather the relative strength of expression of these effects that is found to be affected by model, algorithm and parameter choices. This is important because there is limited consensus on what, even within our general classes methods, the reference method for structural connectome tractography should be. Previous work has used methods in a large subset of our general classes, including MDD (e.g. Hagmann et al., 2008 and Zalesky et al., 2010), SDD (e.g. Zalesky et al., 2010), MDP (e.g. Li et al., 2011), SDG (e.g. Iturria-Medina et al., 2007) and MDG (e.g. Iturria-Medina et al., 2011). There are many intra-voxel diffusion models and tractography algorithms that could be used besides the ones we have chosen here. We listed some of the available alternatives in Table 1 and in the Introduction section. However, rather than performing an exhaustive unstructured test of all or most available methods, we have chosen to examine the effect of three common distinctions that almost any approach is characterized by. These are the distinctions between i) single fiber direction versus multiple directions in the intra-voxel diffusion model, ii) deterministic versus probabilistic tractography and iii) local versus global measure-of-fit tractography. Within each class defined by these distinctions (SDD, MDD, SDP, MDP, SDG and MDG) we chose one of the most used or most common methods as the reference. Obviously, other choices could have been made here. For instance in the SDD class, variants of the deterministic streamline 
algorithm exist which use different local direction finding (such as tensor deflection or TEND), step integration approaches (e.g. $4^{\text {th }}$ order Runge-Kutta) and ODF interpolation techniques. DSI, QBI, and the balland-stick model are all quite commonly used as multi-direction models for tractography and PROBTRACKX (Behrens et al., 2007) is an often used alternative for iFOD for local probabilistic multi-direction tractography. Of course, the particular strategy selected from each general class might not be representative of the class in general in all its varieties and detail. However, for the level of general comparison that we aim at here, the subtle distinctions between methods within a general class listed in Table 1 is not the focus of investigation. In addition, a practical constraint is formed by the need to create a whole brain connection matrix, requiring many thousands of runs of any tractography algorithm, which is computationally challenging especially for probabilistic and global tractography methods.

The effect of algorithm and parameter choice has two components. First, there is the large effect of tractography algorithm and parameters on global network density, which is known to strongly affect graph indices. We have found that connection density generally decreases: i) with increasing probabilistic threshold, ii) when going from probabilistic to deterministic tractography, iii) with increasing FA and decreasing angle thresholds, iv) when moving from global to local tractography, and v) when moving from multi direction to single direction models. Second, more importantly, there are remaining effects on graph indices even when global density is controlled for which range in the tens of percent. It is important to note that maximizing absolute small-worldness is never the aim in quantifying this measure in any given individual or in a comparison. Instead, the aim is to have a robust, specific and sensitive measure of global connection topology in the brain as a useful dependent variable.

A pertinent question that has been addressed, at least in part, in a number of previous studies - though not always in the context of 
connectome reconstruction - is what reasons we have to choose multiple direction models over single, probabilistic over deterministic and global over local tractography. Given the effect on topology measures discussed above, the question of the optimal tractography approach becomes even more important. Therefore, in the second part of this paper we have presented an approach to quality control part of the connectome adjacency matrices which is suitable for comparing the different tractography approaches. Conceivably, it could be used to quality control entire connectome matrices by requiring sensitivity TSDCs in a certain range for a considerable number of known tracts and specificity TSDCs to be low for a number of known not-to-be-connected ROIs, which together span a large fraction of the adjacency matrix. This approach uses connectome dissection into a few large associative bundles which are known to exist and calculation of quantitative TSDCs. This is combined with careful inspection of tractography paths themselves to allow for assessment of true/false positives and true/false negatives. This brings us beyond the mere assessment of global measures, such as graph density or average streamline length, to the actual connections in the brain that are reconstructed by more streamlines or longer streamlines. We can use these results to inform choices in the tractography algorithms. Below we discuss the magnitude and direction of the effects of these choices on global connection topology measures, how these choices interact with each other and with threshold parameters and the signal-to-noise ratio (SNR) of the DWI data, and which choice is favored by the proposed quality control approach.

\section{Single versus multiple directions}

The main effect of moving from single direction intra-voxel diffusion models to multi-direction models is an increase in the number of long streamlines or tractography paths (see Fig. 2 and Table 2 for deterministic algorithms), which is further increased by liberal angle thresholds. Thus, multi-direction models yield higher density and global 
network efficiency and lower small-worldness than single-direction ones at the same percentile threshold (see Supplementary Figs. 1-5). This agrees with other reports of greater network connection density for deterministic tractography on multiple direction models compared to the DTI model (Bassett et al., 2011a and Zalesky et al., 2010). For global approaches, the spatial degree distribution is less focused on high density nodes for MDG, perhaps because longer tractography paths are retained compared to SDG (Fig. 11). When identifying exclusively topological differences by matching graph densities, small-worldness is seen to be larger under some circumstances for multiple directions than single direction, which is explained by a smaller normalized path length, with normalized average clustering coefficient being relatively unaffected.

The connectome dissection $\mathrm{QC}$ is most decisive on the choice of single versus multiple directions in the case of the inter-hemispheric projections through the body of the corpus callosum. In agreement with earlier reports (Behrens et al., 2007, Tuch et al., 2003 and Wedeen et al., 2008), this shows a preference for multiple direction models over single direction ones for tractography. This is motivated by higher TSDCcc and more veridical results for multiple direction models, along with a low number of false positives for appropriate probabilistic thresholds as quantified by the TSDCFr. Thus, this is an interesting example of a justifiable method choice that has the effect of decreasing the expression of high density nodes, since at least part of the increased long range density is formed by true connections that were otherwise missed.

A general point in fitting models to data is clearly exposed in comparing multiple versus single direction models, namely that of model complexity and sensitivity to noise, or of bias and variance. A multi direction model is more sensitive to the SNR of a diffusion weighted dataset and will show greater variability in reconstructed fiber orientations as SNR becomes lower because more parameters must be estimated. This can result in spurious (false positives) or missing (false 
negatives) fiber directions identified in single noisy voxels. The simpler single direction models such as DTI are more robust against noise having much fewer parameters to identify which can work very well in areas with simple fiber architectures such as the SLFII. This might represent a constraint when deciding which model to use for the analysis of diffusion weighted data. When performing a case-control study, for example, a simple single direction DTI-based SDD approach can be preferable compared to an MDD one, when it reduces the intersubject variability within groups explaining more variance at an acceptable cost of delivering more false negatives.

\section{Deterministic versus probabilistic}

With increasing probabilistic threshold adjacency matrix density decreases, as one would expect when eliminating edges by thresholding on probabilistic weights. Generally, this decrease in density is accompanied by higher small-worldness (mostly through increase of normalized averaged clustering coefficient) and lower global network efficiency (see Supplementary Figs. 1-5). Furthermore, for the global algorithms employed here, a higher probabilistic threshold increased the focus of high density nodes, i.e. it increased the sharpness of peaks in the degree distribution over the cortical nodes (Fig. 11). Since probabilistic weights fall off with path length and as evidenced by a higher clustering (a higher relative abundance of local connections), it follows that increasing probabilistic thresholds prunes the network to lower density mostly by removing long range connections.

When holding all other parameters constant, deterministic methods generally capture all the extremes of the effects that were observed for increasing probabilistic threshold. In other words, a 'deterministic limit of probabilistic results' is observed experimentally in the case of edge density and can be considered to form a $100 \%$ limit value for the entire probabilistic threshold curves. Note that this is not meant to imply that the high threshold limit of probabilistic tractography results is necessarily equivalent to that of a deterministic 
algorithm seeded in the same location. The high threshold limit value result for deterministic approaches here only applies to global network characteristics derived from whole brain tractography. When looking at pure topological properties of the network by matching the densities, deterministic algorithms have much larger small-worldness than probabilistic algorithms which is mainly explained by a much shorter characteristic path length. This is true both for a seeding strategy for deterministic algorithms in the entire WM (diamonds in Fig. 3, Fig. 4, Fig. 5 and Fig. 6) and only at the WMbound (squares in Fig. 3, Fig. 4,Fig. 5 and Fig. 6). Normalized average clustering coefficient is less affected by this choice, although differences up to $10 \%$ can be observed.

The conceptual advantage of probabilistic tractography is that it quantifies both average pathways and variation in tractography results (Behrens et al., 2003 and Parker et al., 2003), which is desirable. However, in the context of connectome reconstruction a practical problem is to choose a probabilistic threshold which - as we show here - has great influence on global network statistics. This problem has briefly been touched upon before without extensive investigation by other authors. In Hagmann et al. (2008) and Zalesky et al. (2010) connectome reconstruction was performed with deterministic tractography and an inter-node weight calculation that involves a number of connecting streamlines, node area volume and/or surface and streamline lengths, as was done here for SDD and MDD. In Li et al. (2011), a single probabilistic threshold is chosen by matching the resulting adjacency matrix density to earlier work (Achard and Bullmore, 2007). Finally, some investigations of adjacency matrices derived from both functional MRI data and DWI tractography have employed integration of results over a smaller or larger range of threshold values on fMRI correlations (from 50 th $99.9^{\text {th }}$ percentiles; Achard and Bullmore, 2007) or probabilistic tractography percentile (from $99^{\text {th }}$ to $99.9^{\text {th }}$ percentiles; Gong et al., 2009). This reveals that either by deterministic fiber reconstruction or by selection of 
relatively high probabilistic thresholds most work has strived for sparse networks (low density) along the continuum sketched in Fig. 1.

An absolute probabilistic threshold independent of a desired final density level is difficult to set since probabilistic tractography visitation counts or weights are hard to interpret in an absolute way (Jbabdi and Johansen-Berg, 2011, Jones, 2008a and Jones, 2010). First, probabilistic weights for streamlines or paths fall off naturally with path length, which in a large part explains the longer characteristic path length compared to density matched adjacency matrices obtained by deterministic algorithms. Second, a probabilistic weight quantifies a mixture of noise in the data (affecting precision of the result), bias in the employed model and the true likelihood of a connection (affecting accuracy of the result). Subsets of these challenges have started to be addressed by Monte Carlo or bootstrap approaches (Jeurissen et al., 2011,Jones, 2008b and Morris et al., 2008). However, these methods are computationally very expensive, even for a single seeding point or ROI, and applying them to connectome reconstruction provides a tremendous computational challenge. Interestingly, the connectome dissection QC approach outlined here offers a quantitative way to define relatively narrow range of tolerable probabilistic threshold for connectome tractography. This is the range of values which simultaneously offers good sensitivity and veridicality of connections (sensitivity TSDCs between 0.1 and 0.5 ) and good specificity (specificity TSDCs below 0.01). In the example of the transcallosal projections this leads to a tolerable range between the $91^{\text {st }}$ and the $98^{\text {th }}$ percentiles, which agrees to good qualitative delineation of these connections (see Fig. 10).

In addition to the effect of probabilistic threshold on tractography path length, there is also an effect of different tractography seeding strategies. Seeding from all of white matter is biased toward longer fiber bundles because they are sampled with a greater number of seed points, whereas seeding from the boundary is biased toward 
recovering shorter bundles ( $\mathrm{Li}$ et al., 2011). We have equalized this seeding bias between tractography algorithms by using the same boundary seeding strategy for all methods (squares in Fig. 3, Fig. 4, Fig. 5 and Fig. 6 for SDD and MDD).

Again there are considerations of model complexity and sensitivity to noise. Local probabilistic methods at very liberal thresholds, especially a 90 degree angle threshold in absence of a colinearity constraint based on prior identification of main diffusion directions can lead to degeneration of algorithm performance and pathological results. This is understandable, given that less restrictive thresholds (FA and angle) decrease modeling assumptions and resulting biases (such as: 'low curvature is good') but also increase variability in the results through the effects of noise. As discussed above, this effect is more pronounced for more complex models with more parameters, such as multi direction models compared to single direction models (Fig. 9). Pathological noise-dominated results can occur when this complexity and lack of constraints is then combined with the controlled variability introduced at each step by local probabilistic algorithms. Among the range of combination of methods and parameters settings, the combination of local probabilistic algorithms with a $90^{\circ}$ angle threshold without a colinearity constraint between fiber direction and local diffusion direction (essentially a low-curvature bias) is certainly an extreme one which is rarely employed in practice. However, it is important to point out that the main reason to use a limited maximum angle (minimum curvature) is not necessarily biologically inspired: nerve bundles and white matter fascicles sometimes can turn sharply (e.g. Roebroeck et al., 2008). Rather, it is the regularizing and smoothing effect of the low-curvature assumption that helps in avoiding sensitivity to excessive noise effects. Therefore, it is interesting to construct and evaluate tractography algorithms that achieve a high robustness to noise with fewer constraints. 


\section{Local versus global}

Going from a local to a global approach affects the estimation of the reconstructed fiber paths in two important ways. First, global methods lead to a higher connection density than their local probabilistic counterparts at the same percentile thresholds (see Fig. 1), likely due to a higher number of longer connections. This means that to match density between global and local probabilistic algorithms implies applying a relatively high percentile threshold to the global and a relatively low one to the local. Nevertheless, when density matched, local shows higher small-worldness than global explained in a large part by a shorter characteristic path length. This is because all long tractography paths have been suppressed in the global results because of the comparatively high threshold. Second, in the connectome dissection QC, the sensitivity/specificity trade-off of the reconstructed fiber paths is qualitatively better for global methods than for the local deterministic ones with the same parameters. For instance, in Fig. 9 and Fig. 10 global methods allow robust tractography even for very liberal thresholds, such as a $90^{\circ}$ angle threshold, which is reflected in a high TSDC $c$ in conjunction with high specificity (low TSDCFP) for appropriate probabilistic thresholds. This decrease in vulnerability to local noise is actually the motivating property of global tractography methods (Iturria-Medina et al., 2007, Jbabdi et al., 2007, Sherbondy et al., 2009 and Sotiropoulos et al., 2010). In addition, the weighted shortestpath finding approach still discourages very long-range connecting fibers (as local probabilistic methods do) which is often a biologically plausible constraint in the brain that can avoid false positives. See, for example, the false positive connections between left and right lateral frontal cortices via the brainstem in SDD and MDD in Fig. 9 that are absent for SDG and MDG in Fig. 10. All in all, global algorithms show higher TSDCs implying higher sensitivity over large ranges of percentile thresholds, as shown in the TSDCCc and TSDCsLFI. Crucially, this is in conjunction with high specificity (very low TSDCFP) for probabilistic 
threshold between approximately the $91^{\text {st }}$ and the $98^{\text {th }}$ percentiles. It is only when comparing to deterministic tractography at very high thresholds (which match TSDCs between methods) that their short path bias weights in strongly as seen for instance in Fig. 12. Here it is important to note that when not trying to match density or TSDC to a deterministic algorithm, there is no a-priori reason to apply such high threshold to global probabilistic results. Finally, the example of the ILF shows interesting differences in behavior between SDD, MDD and global algorithms. In this case, where identification of local direction is imperfect, TSDC for SDD is the same as for the other tracts, TSDC for MDD is reduced by a factor 2 to a factor 3 and TSDC for SDG and MDG is decreased by an order of magnitude. This shows that for a combination of irregularities in the data and a long tract, global algorithms can show a decrease in performances which is, however, picked up very well by the connectome dissection QC. Although the performance of SDD is very consistent across different tracts, it is important to remark that it shows a consistently low sensitivity. For instance, the TSDCSLFII and TSDCILF are as low as for the clearly underconnected CC (see Fig. 9), despite specificity for SLFII (see Fig. 12) and ILF (see Fig. 13) being rather good.

Two disadvantages of global methods must be mentioned. First, there is their high computational cost. Probabilistic methods, global even more than local approaches, were the most time consuming methods to run for the very large whole-brain tractography sets. In addition, this computational cost will increase more than linearly with increase in spatial resolution of the DWI data as MR hardware and sequences advance (e.g. Feinberg et al., 2010 and Heidemann et al., 2010). Second, to keep computational complexity within reasonable bounds (in the limit: to avoid trying to exhaustively cover an infinity of options), global algorithms need constraints on the allowable shapes of pathways. This can be achieved either by discretizing a parameterization (the so-called Hough-transform) of continuous curves 
through the data (such as polynomials of a given order; Aganj et al., 2011) or by discretizing the allowable curves on a $3 D$ grid as in the graph-based tractography algorithms employed here. As a consequence, from a local point of view, both the step size and the angle of local curvature in moving from one gridpoint to the next are constrained and anisotropic. That is, in the $5 \times 5 \times 5$ local neighborhood used here there are 98 discrete unique directions to move in with a few different step sizes. It is important to note that this is indeed a very 'local streamline' way of looking at a global algorithm, since local possibly erroraccumulating steps are never actually performed in graph tractography. Rather, the complete enumeration of all possible discrete traversals through the entire dataset is searched. In this search for a shortest weighted path the local weights are defined by the DWI data modeling using the entire solid angle around a step direction such that any directional peak in the data is taken into account (Iturria-Medina et al., 2007 and Sotiropoulos et al., 2010). Nevertheless, it can be appreciated in Fig. 10, Fig. 12 and Fig. 13 that this approach will lead to fiber pathways that are more discrete in nature. Thus, given the combined advantages of multi-direction models and global tractography, investigation into computationally feasible global approaches with a minimum of pathway shape constraints may prove useful for whole brain structural network reconstruction studies.

\section{Conclusions}

We have shown that general choices in tractography algorithm in combination with the employed thresholds on FA, angle and probabilistic percentiles have a large effect on derived human structural brain network indices. This effect has two components. First, there is the large effect of tractography algorithm and parameters on global network density, which is known to strongly affect graph indices. Second, more importantly, there are remaining effects even when global density is controlled for. Although small-worldness, global network efficiency and 
density are a robust finding over all investigated methods and parameters, their absolute strength of expression can be changed by several tens of percent, which is significant in their role as a sensitive and accurate metric to distinguish subtle differences in global network topology. Since maximizing the absolute value of network indices is not the aim in itself, the reasons we have to choose multiple fiber direction models over single direction models, probabilistic over deterministic and global over local tractography methods are more subtle.

We investigate this with the proposed connectome dissection QC in which evaluation of TSDCs measure sensitivity and specificity in combination with careful inspection of tractography path results. Conceivably, it could be used within a semi-automated quality control framework for entire connectome matrices by requiring sensitivity TSDCs in a certain range for a considerable number of known tracts and specificity TSDCs to be low for a number of known not-to-be-connected ROIs, which together span a large fraction of the adjacency matrix. This would require development or application of an atlas of such end-ROI which can be mapped to individual datasets and maturation of conventions for tolerable TSDC value limits, which is an important topic of future work.

Here, the results on sensitivity and specificity for a subset of tractography paths corroborate earlier investigations that conclude a superiority of multiple direction models for intra-voxel fiber direction modeling. More tentatively, probabilistic tractography might be preferred over deterministic for its capacity to quantify the variability of tracking results. However, the choice of probabilistic threshold in the context of whole brain structural network delineation still remains an open issue of importance. Interestingly, the connectome dissection QC approach outlined here offers a quantitative way to define relatively narrow range of tolerable probabilistic threshold for connectome tractography. This is the range of values which simultaneously offers good sensitivity and veridicality of connections (sensitivity TSDCs 
between 0.1 and 0.5 ) and good specificity (specificity TSDCs below 0.01). Fig. 8 nicely shows that in this way TSDCs can quantify sensitivity (CC and SLFII), lack of sensitivity (ILF) and specificity (FP). Probabilistic local tractography algorithms can exhibit considerable noise sensitivity when applied to multi direction models without strong constraints on local tract curvature. Therefore, global approaches that exhibit less sensitivity to local noise may be preferred, particularly when more of the available SNR is used in the DWI acquisition to improve spatial resolution. Development of global tractography methods that balance reasonable computational demands with acceptable constraints aimed at limiting the search space (such as discretization) might further improve their applicability.

\section{Acknowledgments}

The authors would like to thank Alexandros Goulas, Federico de Martino, Kâmil Uludağ and Yasser Iturria-Medina for helpful comments and suggestions on earlier versions of this manuscript. They would also like to thank two reviewers for valuable suggestions which have significantly improved this manuscript. This work was funded by EU Marie Curie Initial Training Network Grant PITN-GA-2009-238593. 


\section{References}

Achard, S., Bullmore, E., 2007. Efficiency and cost of economical brain functional networks. PLoS Comput. Biol. 3, e17.

Aganj, I., Lenglet, C., Jahanshad, N., Yacoub, E., Harel, N., Thompson, P.M., Sapiro, G., 2011. A Hough transform global probabilistic approach to multiple-subject diffusion MRI tractography. Med. Image Anal. 15, 414-425.

Assaf, Y., Basser, P.J., 2005. Composite hindered and restricted model of diffusion (CHARMED) MR imaging of the human brain. NeuroImage 27, 48-58.

Basser, P.J., Pierpaoli, C., 1996. Microstructural and physiological features of tissues elucidated by quantitative-diffusion-tensor MRI. J. Magn. Reson. B 111, 209-219.

Basser, P.J., Mattiello, J., LeBihan, D., 1994. MR diffusion tensor spectroscopy and imaging. Biophys. J. 66, 259-267.

Basser, P.J., Pajevic, S., Pierpaoli, C., Duda, J., Aldroubi, A., 2000. In vivo fiber tractography using DT-MRI data. Magn. Reson. Med. 44, 625-632.

Bassett, D.S., Brown, J.A., Deshpande, V., Carlson, J.M., Grafton, S.T., 2011a. Conserved and variable architecture of human white matter connectivity. NeuroImage 54, 1262 1279.

Bassett, D.S., Wymbs, N.F., Porter, M.A., Mucha, P.J., Carlson, J.M., Grafton, S.T., 2011b. Dynamic reconfiguration of human brain networks during learning. Proc. Natl. Acad. Sci. U. S. A. 108, 7641-7646.

Behrens, T.E., Woolrich, M.W., Jenkinson, M., Johansen-Berg, H., Nunes, R.G., Clare, S., Matthews, P.M., Brady, J.M., Smith, S.M., 2003. Characterization and propagation of uncertainty in diffusion-weighted MR imaging. Magn. Reson. Med. 50, 1077-1088.

Behrens, T.E., Berg, H.J., Jbabdi, S., Rushworth, M.F., Woolrich, M.W., 2007. Probabilistic diffusion tractography with multiple fibre orientations: what can we gain? NeuroImage 34, 144-155.

Cammoun, L., Gigandet, X., Meskaldji, D., Thiran, J.P., Sporns, O., Do, K.Q., Maeder, P., Meuli, R., Hagmann, P., 2012. Mapping the human connectome at multiple scales with diffusion spectrum MRI. J. Neurosci. Methods 203, 386-397.

Catani, M., Thiebaut de Schotten, M., 2008. A diffusion tensor imaging tractography atlas for virtual in vivo dissections. Cortex 44, 1105-1132.

Cheng, H., Wang, Y., Sheng, J., Sporns, O., Kronenberger, W.G., Mathews, V.P., Hummer, T.A., Saykin, A.J., 2011. Optimization of seed density in DTI tractography for structural networks. J. Neurosci. Methods 203 (1), 264-272.

Conturo, T.E., Lori, N.F., Cull, T.S., Akbudak, E., Snyder, A.Z., Shimony, J.S., McKinstry, R.C., Burton, H., Raichle, M.E., 1999. Tracking neuronal fiber pathways in the living human brain. Proc. Natl. Acad. Sci. U. S. A. 96, 10422-10427. 
Cook, P.A., Bai, Y., Nedjati-Gilani, S., Seunarine, K.K., Hall, M.G., Parker, G.J., Alexander, D.C., 2006. Camino: open-source diffusion-MRI reconstruction and processing. 14th Scientific Meeting of the International Society for Magnetic Resonance in Medicine, Seattle, WA, USA.

Du, Q., Gunzburger, M.D., Ju, L.L., 2003. Constrained centroidal Voronoi tessellations for surfaces. SIAM J. Sci. Comput. 24, 1488-1506.

Feinberg, D.A., Moeller, S., Smith, S.M., Auerbach, E., Ramanna, S., Gunther, M., Glasser, M.F., Miller, K.L., Ugurbil, K., Yacoub, E., 2010. Multiplexed echo planar imaging for sub-second whole brain FMRI and fast diffusion imaging. PLoS One 5, e15710.

ffytche, D.H., Blom, J.D., Catani, M., 2010. Disorders of visual perception. J. Neurol. Neurosurg. Psychiatry 81, 1280-1287.

Fillard, P., Descoteaux, M., Goh, A., Gouttard, S., Jeurissen, B., Malcolm, J., RamirezManzanares, A., Reisert, M., Sakaie, K., Tensaouti, F., Yo, T., Mangin, J.F., Poupon, C., 2011. Quantitative evaluation of 10 tractography algorithms on a realistic diffusion MR phantom. NeuroImage 56, 220-234.

Frank, L.R., 2001. Anisotropy in high angular resolution diffusion-weighted MRI. Magn. Reson. Med. 45, 935-939.

Goebel, R., Esposito, F., Formisano, E., 2006. Analysis of functional image analysis contest (FIAC) data with BrainVoyager QX: from single-subject to cortically aligned group general linear model analysis and self-organizing group independent component analysis. Hum. Brain Mapp. 27, 392-401.

Gong, G., Rosa-Neto, P., Carbonell, F., Chen, Z.J., He, Y., Evans, A.C., 2009. Age- and gender related differences in the cortical anatomical network. J. Neurosci. 29, 1568415693.

Hagmann, P., Kurant, M., Gigandet, X., Thiran, P., Wedeen, V.J., Meuli, R., Thiran, J.P., 2007. Mapping human whole-brain structural networks with diffusion MRI. PLoS One 2, e597.

Hagmann, P., Cammoun, L., Gigandet, X., Meuli, R., Honey, C.J., Wedeen, V.J., Sporns, O., 2008. Mapping the structural core of human cerebral cortex. PLoS Biol. 6, e159.

Heidemann, R.M., Porter, D.A., Anwander, A., Feiweier, T., Heberlein, K., Knosche, T.R., Turner, R., 2010. Diffusion imaging in humans at 7T using readout-segmented EPI and GRAPPA. Magn. Reson. Med. 64, 9-14.

Iturria-Medina, Y., Canales-Rodriguez, E.J., Melie-Garcia, L., Valdes-Hernandez, P.A., Martinez-Montes, E., Aleman-Gomez, Y., Sanchez-Bornot, J.M., 2007. Characterizing brain anatomical connections using diffusion weighted MRI and graph theory. NeuroImage 36, 645-660.

Iturria-Medina, Y., Perez Fernandez, A., Morris, D.M., Canales-Rodriguez, E.J., Haroon, H.A., Garcia Penton, L., Augath, M., Galan Garcia, L., Logothetis, N., Parker, G.J., Melie- 
Garcia, L., 2011. Brain hemispheric structural efficiency and interconnectivity rightward asymmetry in human and nonhuman primates. Cereb. Cortex 21, 56-67.

Jarbo, K., Verstynen, T., Schneider, W., 2012. In vivo quantification of global connectivity in the human corpus callosum. NeuroImage 59, 1988-1996.

Jbabdi, S., Johansen-Berg, H., 2011. Tractography: where do we go from here? Brain Connect. 1, 169-183.

Jbabdi, S., Woolrich, M.W., Andersson, J.L., Behrens, T.E., 2007. A Bayesian framework for global tractography. NeuroImage 37, 116-129.

Jeurissen, B., Leemans, A., Jones, D.K., Tournier, J.D., Sijbers, J., 2011. Probabilistic fiber tracking using the residual bootstrap with constrained spherical deconvolution. Hum. Brain Mapp. 32, 461-479.

Jones, D.K., 2008a. Studying connections in the living human brain with diffusion MRI. Cortex 44, 936-952.

Jones, D.K., 2008b. Tractography gone wild: probabilistic fibre tracking using the wild bootstrap with diffusion tensor MRI. IEEE Trans. Med. Imaging 27, 1268-1274.

Jones, D.K., 2010. Challenges and limitations of quantifying brain connectivity in vivo with diffusion MRI. Imaging Med. 2, 341-355.

Lazar, M., Weinstein, D.M., Tsuruda, J.S., Hasan, K.M., Arfanakis, K., Meyerand, M.E., Badie, B., Rowley, H.A., Haughton, V., Field, A., Alexander, A.L., 2003. White matter tractography using diffusion tensor deflection. Hum. Brain Mapp. 18, 306-321.

Leemans, A., Jones, D.K., 2009. The B-matrix must be rotated when correcting for subject motion in DTI data. Magn. Reson. Med. 61, 1336-1349.

Li, L., Rilling, J.K., Preuss, T.M., Glasser, M.F., Hu, X., 2011. The effects of connection reconstruction method on the interregional connectivity of brain networks via diffusion tractography. Hum. Brain Mapp. (Electronic publication ahead of print) http:// dx.doi.org/10.1002/hbm.21332.

Liu, C., Bammer, R., Acar, B., Moseley, M.E., 2004. Characterizing non-Gaussian diffusion by using generalized diffusion tensors. Magn. Reson. Med. 51, 924-937.

Mori, S., Crain, B.J., Chacko, V.P., van Zijl, P.C., 1999. Three-dimensional tracking of axonal projections in the brain by magnetic resonance imaging. Ann. Neurol. 45, 265269.

Morris, D.M., Embleton, K.V., Parker, G.J., 2008. Probabilistic fibre tracking: differentiation of connections from chance events. NeuroImage 42, 1329-1339.

Ozarslan, E., Mareci, T.H., 2003. Generalized diffusion tensor imaging and analytical relationships between diffusion tensor imaging and high angular resolution diffusion imaging. Magn. Reson. Med. 50, 955-965.

Ozarslan, E., Shepherd, T.M., Vemuri, B.C., Blackband, S.J., Mareci, T.H., 2006. Resolution of complex tissue microarchitecture using the diffusion orientation transform (DOT). NeuroImage 31, 1086-1103. 
Parker, G.J., Alexander, D.C., 2005. Probabilistic anatomical connectivity derived from the microscopic persistent angular structure of cerebral tissue. Philos. Trans. R. Soc. Lond. B Biol. Sci. 360, 893-902.

Parker, G.J., Haroon, H.A., Wheeler-Kingshott, C.A., 2003. A framework for a streamlinebased probabilistic index of connectivity (PICo) using a structural interpretation of MRI diffusion measurements. J. Magn. Reson. Imaging 18, 242-254.

Ramayya, A.G., Glasser, M.F., Rilling, J.K., 2010. A DTI investigation of neural substrates supporting tool use. Cereb. Cortex 20, 507-516.

Roebroeck, A., Galuske, R., Formisano, E., Chiry, O., Bratzke, H., Ronen, I., Kim, D.S., Goebel, R., 2008. High-resolution diffusion tensor imaging and tractography of the human optic chiasm at $9.4 \mathrm{~T}$. NeuroImage $39,157-168$.

Rubinov, M., Sporns, O., 2010. Complex network measures of brain connectivity: uses and interpretations. NeuroImage 52, 1059-1069.

Schultz, T., Seidel, H.P., 2008. Estimating crossing fibers: a tensor decomposition approach. IEEE Trans. Vis. Comput. Graph. 14, 1635-1642.

Sherbondy, A.J., Dougherty, R.F., Ben-Shachar, M., Napel, S., Wandell, B.A., 2008. ConTrack: finding the most likely pathways between brain regions using diffusion tractography. J. Vis. 8 (15), 11-16.

Sherbondy, A.J., Dougherty, R.F., Ananthanarayanan, R., Modha, D.S., Wandell, B.A., 2009. Think global, act local; projectome estimation with BlueMatter. Med. Image Comput. Comput. Assist. Interv. 12, 861-868.

Sotiropoulos, S.N., Bai, L., Morgan, P.S., Constantinescu, C.S., Tench, C.R., 2010. Brain tractography using Q-ball imaging and graph theory: improved connectivities through fibre crossings via a model-based approach. NeuroImage 49, 2444-2456.

Sporns, O., Tononi, G., Kotter, R., 2005. The human connectome: a structural description of the human brain. PLoS Comput. Biol. 1, e42.

Thiebaut de Schotten, M., Dell'Acqua, F., Forkel, S.J., Simmons, A., Vergani, F., Murphy, D.G., Catani, M., 2011. A lateralized brain network for visuospatial attention. Nat. Neurosci. 14, 1245-1246.

Tournier, J.D., Calamante, F., Gadian, D.G., Connelly, A., 2004. Direct estimation of the fiber orientation density function from diffusion-weighted MRI data using spherical deconvolution. NeuroImage 23, 1176-1185.

Tournier, J.D., Calamante, F., Connelly, A., 2007. Robust determination of the fibre orientation distribution in diffusion MRI: non-negativity constrained super-resolved spherical deconvolution. NeuroImage 35, 1459-1472.

Tournier, J.D., Calamante, F., Connelly, A., 2010. Improved probabilistic streamlines tractography by 2nd order integration over fibre orientation distributions. Proceedings of the Joint Annual Meeting ISMRM-ESMRMB 2010, Stockholm, Sweden.

Tuch, D.S., 2004. Q-ball imaging. Magn. Reson. Med. 52, 1358-1372. 
Tuch, D.S., Reese, T.G., Wiegell, M.R., Makris, N., Belliveau, J.W., Wedeen, V.J., 2002. High angular resolution diffusion imaging reveals intravoxel white matter fiber heterogeneity. Magn. Reson. Med. 48, 577-582.

Tuch, D.S., Reese, T.G., Wiegell, M.R., Wedeen, V.J., 2003. Diffusion MRI of complex neural architecture. Neuron 40, 885-895.

van Wijk, B.C., Stam, C.J., Daffertshofer, A., 2010. Comparing brain networks of different size and connectivity density using graph theory. PLoS One 5, e13701.

Wakana, S., Jiang, H., Nagae-Poetscher, L.M., van Zijl, P.C., Mori, S., 2004. Fiber tract based atlas of human white matter anatomy. Radiology 230, 77-87.

Watts, D.J., Strogatz, S.H., 1998. Collective dynamics of 'small-world' networks. Nature 393, 440-442.

Wedeen, V.J., Hagmann, P., Tseng, W.Y., Reese, T.G., Weisskoff, R.M., 2005. Mapping complex tissue architecture with diffusion spectrum magnetic resonance imaging. Magn. Reson. Med. 54, 1377-1386.

Wedeen, V.J.,Wang, R.P., Schmahmann, J.D., Benner, T., Tseng, W.Y., Dai, G., Pandya, D.N., Hagmann, P., D'Arceuil, H., de Crespigny, A.J., 2008. Diffusion spectrum magnetic resonance imaging (DSI) tractography of crossing fibers. NeuroImage 41, 1267-1277.

Westin, C.F., Maier, S.E., Mamata, H., Nabavi, A., Jolesz, F.A., Kikinis, R., 2002. Processing and visualization for diffusion tensor MRI. Med. Image Anal. 6, 93-108.

Yeh, F.C., Wedeen, V.J., Tseng, W.Y., 2010. Generalized q-sampling imaging. IEEE Trans. Med. Imaging 29, 1626-1635.

Zalesky, A., 2008. DT-MRI fiber tracking: a shortest paths approach. IEEE Trans. Med. Imaging 27, 1458-1471.

Zalesky, A., Fornito, A., 2009. A DTI-derived measure of cortico-cortical connectivity. IEEE Trans. Med. Imaging 28, 1023-1036.

Zalesky, A., Fornito, A., Harding, I.H., Cocchi, L., Yucel, M., Pantelis, C., Bullmore, E.T., 2010. Whole-brain anatomical networks: does the choice of nodes matter? NeuroImage 50, 970-983.

Zhang, Y., Brady, M., Smith, S., 2001. Segmentation of brain MR images through a hidden Markov random field model and the expectation-maximization algorithm. IEEE Trans. Med. Imaging 20, 45-57. 


\section{Appendix A}

\section{Parcellation scheme}

The equal-area surface parcellation procedure used in this work is based on constrained centroidal Voronoi tessellations (Du et al., 2003). This approach relies on the iterative Lloyd's algorithm, which is often used in computer science to produce point distributions over two or threedimensional surfaces with blue noise characteristics. A blue noise distribution ensures uniformity and regularity in the sampling without showing any bias in the Fourier spectrum.

The proposed algorithm uses Voronoi patches to sub-divide the initial surface into n different ROIs. By definition, a Voronoi patch comprises all the points which are closest to its centroid. Initially, a Voronoi tessellation is used whose centroids are randomly defined (white noise distribution) over the two dimensional surface. Iteratively, the algorithm shifts the centroid of each cell to its barycenter by minimizing the following equation:

$$
\min _{z \in S} \int_{V} \rho(x)|x-z|^{2} d x
$$

where $\mathrm{z}$ is the new centroid, $\mathrm{x}$ are the points comprised in a certain Voronoi patch, $\mathrm{S}$ is the surface, $\mathrm{V}$ is the Voronoi patch and $\mathrm{Q}$ is a density function, here uniformly set to 1 . Note that, since finding the exact barycenter on a 2D surface based on geodesic distances is an NP hard problem, in the proposed algorithm it is always confined to be one of the vertices in the patch and, therefore, trivially lies on the $2 \mathrm{D}$ surface. This approach, being more computationally tractable, uses an approximate estimate of the real barycenter. At every step, the algorithm computes the geodesic distances between all the surface points and the neighbors of the current barycenter of a certain patch. The new barycenter is the neighbor point which minimizes the proposed equation. 
The distances between the centroids and the other points are not 3D Euclidean but geodesics along the 2D surface. The algorithm converges to make the areas of the patches as close to each other as possible. 


\section{Appendix B}

\section{Supplementary Material}
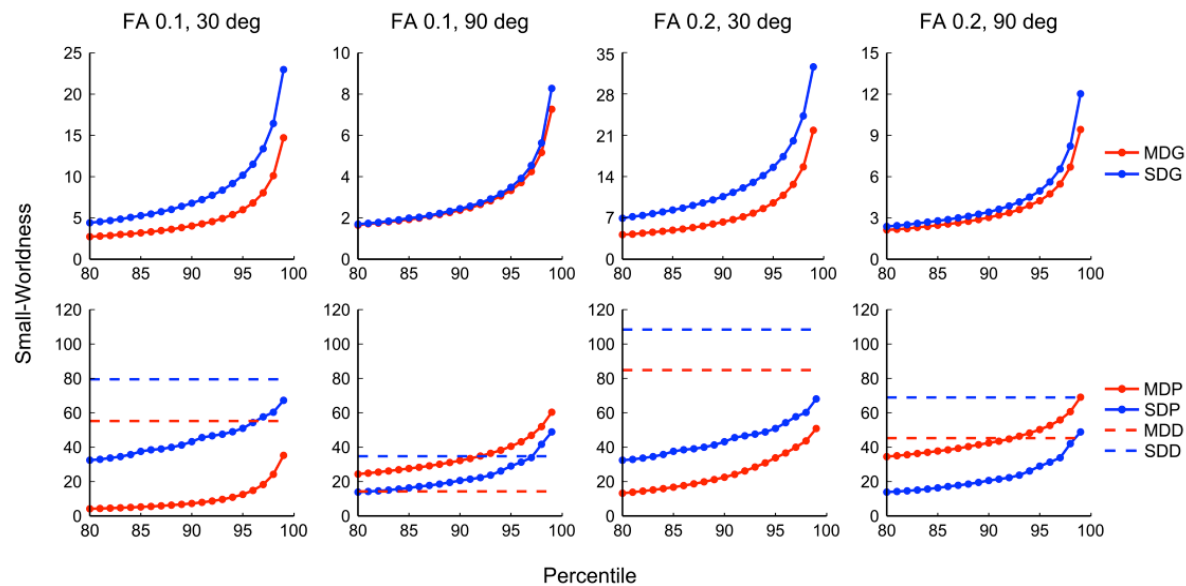

Figure S1: Small-worldness averaged over 2 subjects and 10 random parcellations plotted against probabilistic percentile threshold. The columns represent different combinations of anisotropy and angle thresholds. Deterministic methods are plotted as a single straight line. Single-direction methods are plotted in blue, multiple-direction methods in red. Note the different scales on the y-axis.
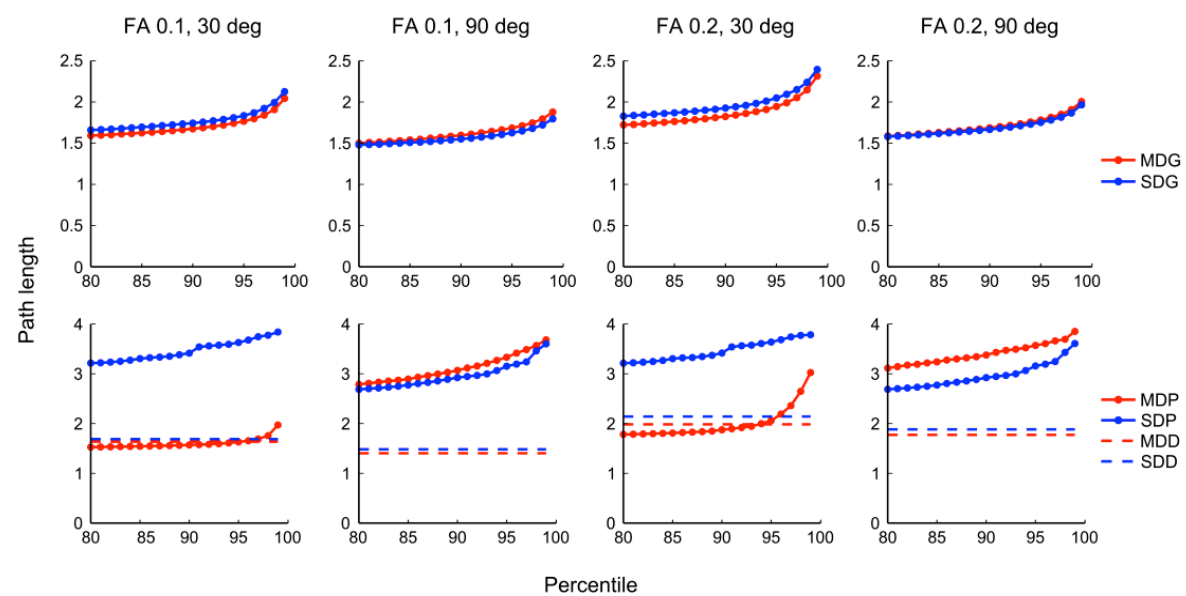

Figure S2: Normalized path length averaged over subjects and random parcellations, organized as in Figure S1. 

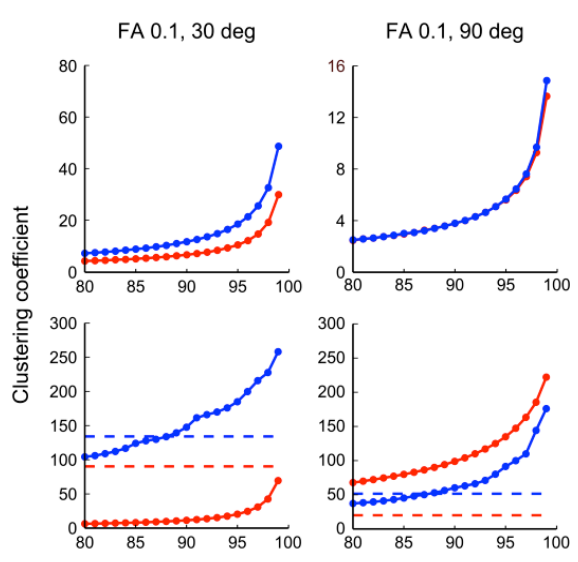

FA $0.2,30 \mathrm{deg}$

FA $0.2,90$ deg
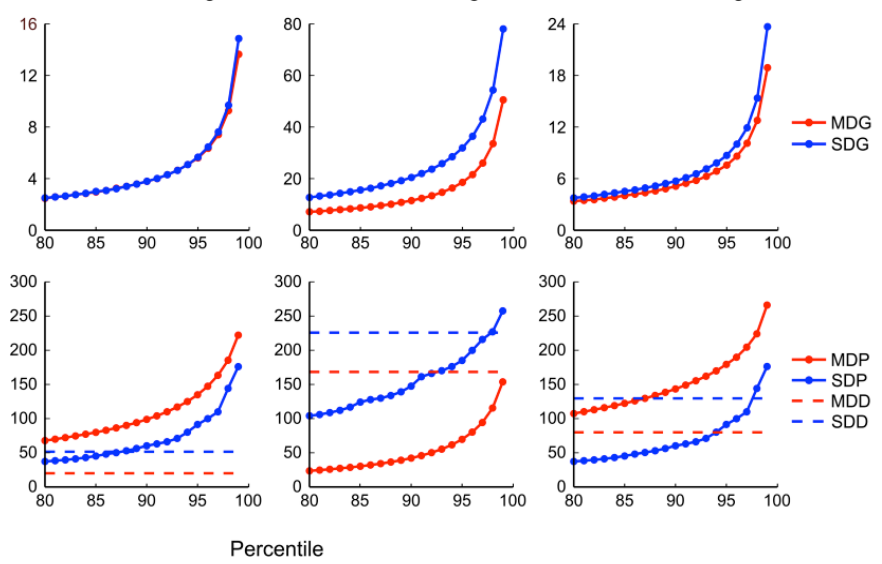

Figure S3: Normalized average clustering coefficient averaged over subjects and random parcellations, organized as in Figure S1.
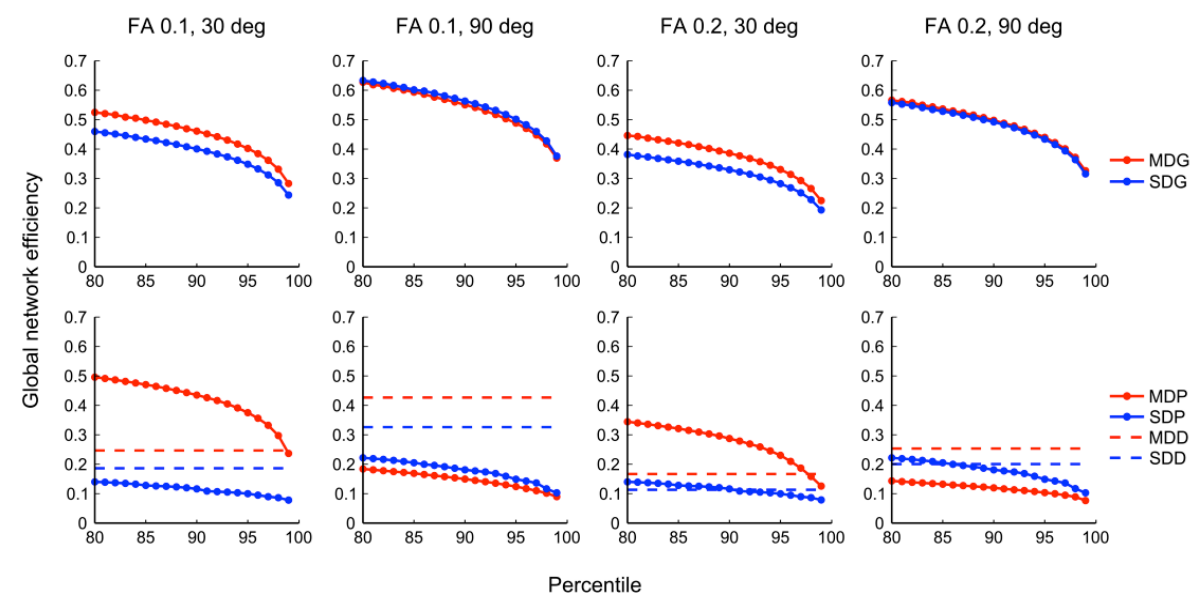

Figure S4: Global network efficiency averaged over subjects and random parcellations, organized as in Figure S1. 


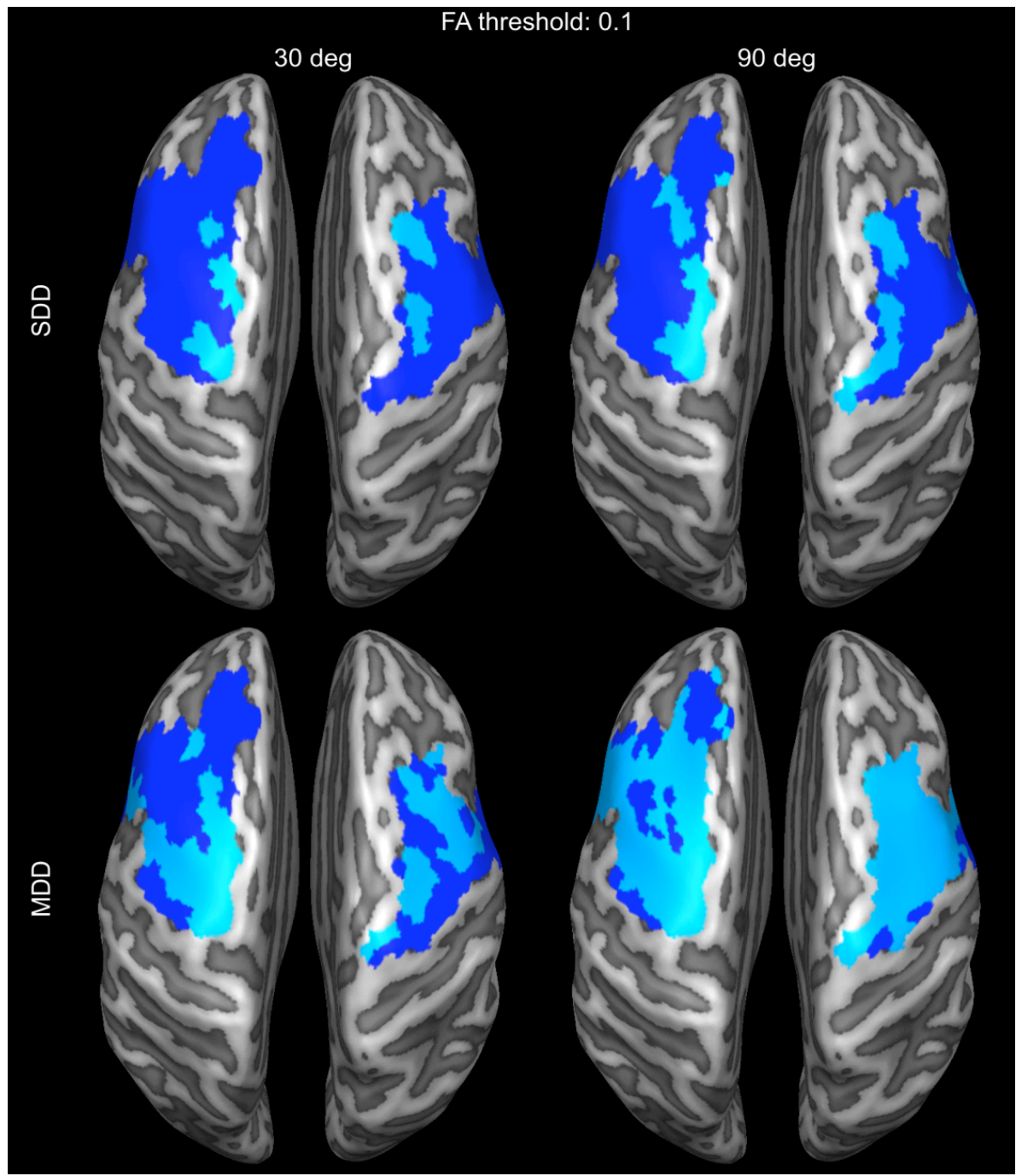

Figure S5: Spatial distribution of connected cortical patches belonging to the two CC ROIs on the left and right lateral frontal cortex in subject 2 for SDD (top row) and MDD (bottom row) at FA threshold 0.1 and two different angle thresholds. Darker color indicates the selected cortical ROI, while brighter color indicates those patches which are connected to each other. 


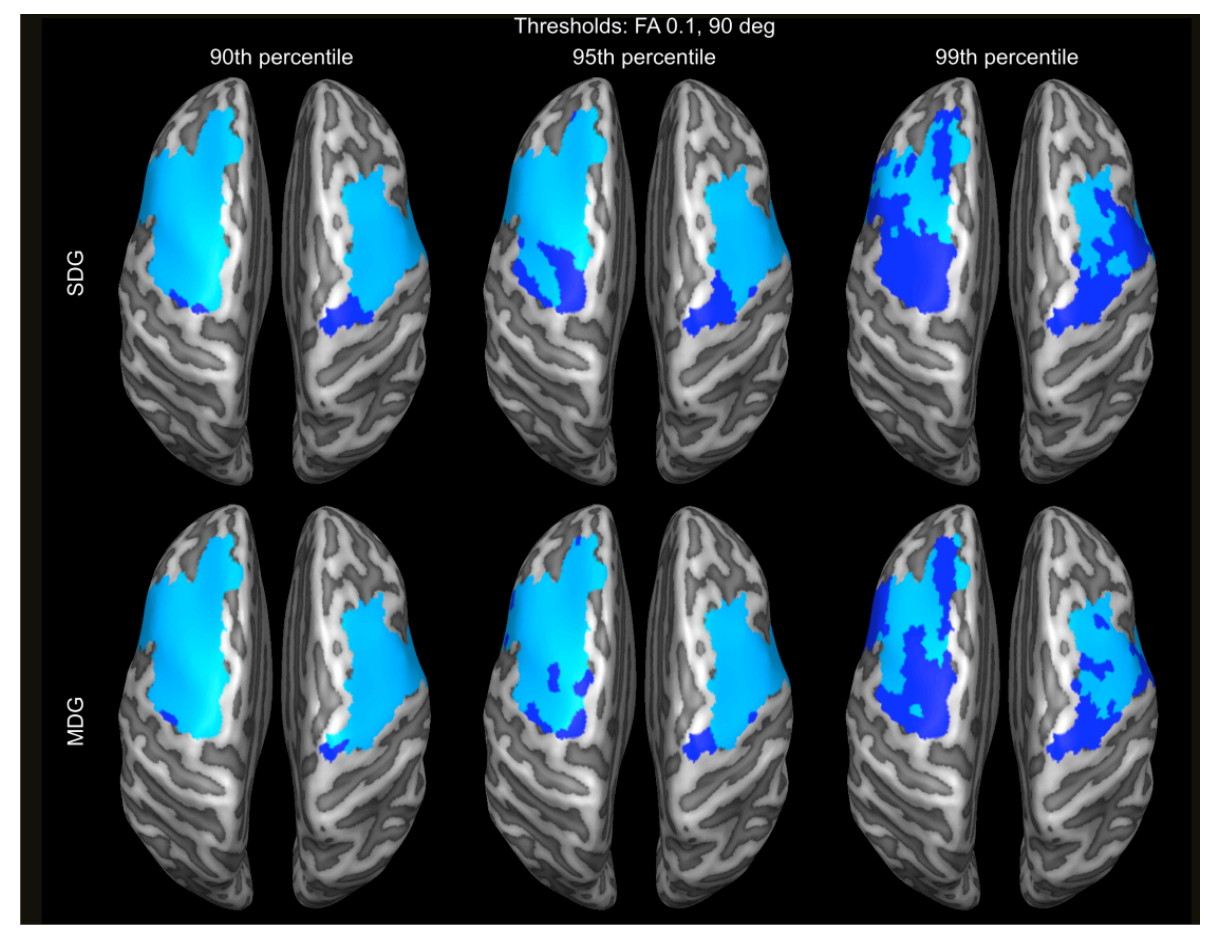

Figure S6: Spatial distribution of connected cortical patches belonging to the two CC ROIs on the left and right lateral frontal cortex in subject 2 for SDG (top row) and MDG (bottom row) at FA threshold 0.1 and angle threshold of 90 degrees. Form left to right, probabilistic thresholds are set to the 90th, 95th and 99th percentiles. Darker color indicates the selected cortical ROI, while brighter color indicates those patches which are connected to each other. 


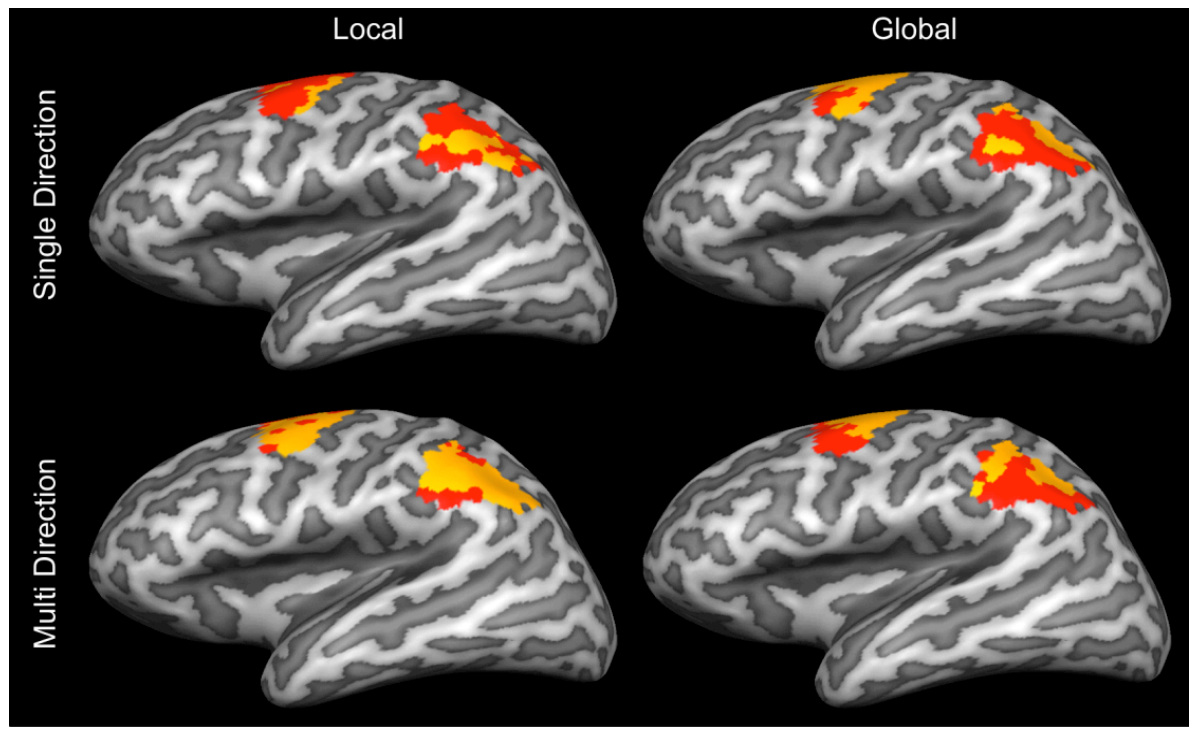

Figure S7: Comparison between spatial distributions of connected cortical patches belonging to the two SLFII ROIs in subject 2 using two different tractography approaches and the same thresholding parameters $(\mathrm{FA}=0.1$, angle threshold 90 degrees). Columns represent the type of tractography approach (left: local deterministic, right: global), rows represent the intra-voxel diffusion model employed (top: single direction DTI, bottom: multi direction CSD). Probabilistic thresholds for global approaches are obtained from Figure 8 and are set to the $99^{\text {th }}$ percentile. All paths are reconstructed using FA 0.1 and 90 degrees as thresholds. Darker color indicates the selected cortical ROI, while brighter color indicates those patches which are connected to each other. 


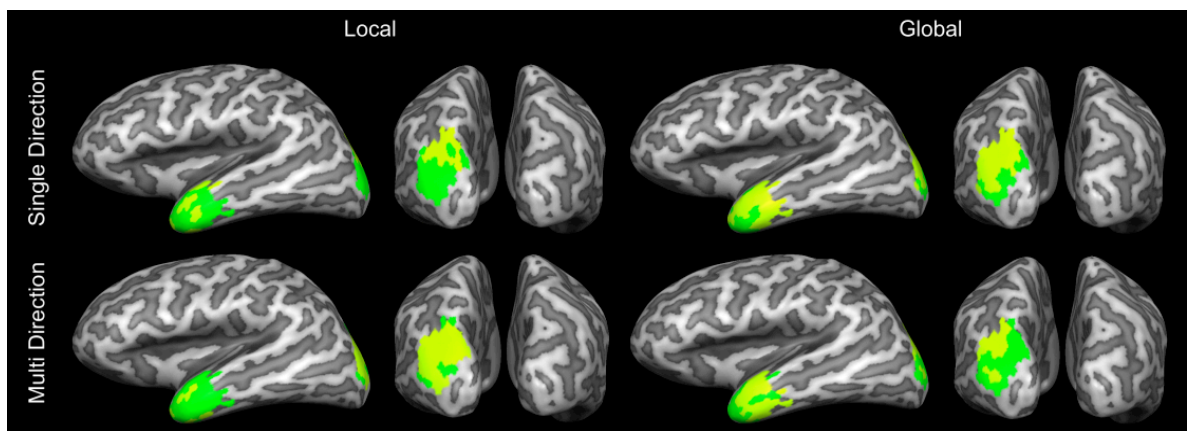

Figure S8: Comparison between spatial distributions of connected cortical patches belonging to the two ILF ROIs in subject 2 using two different tractography approaches and the same thresholding parameters ( $\mathrm{FA}=0.1$, angle threshold 90 degrees). Columns represent the type of tractography approach (left: local deterministic, right: global), rows represent the intra-voxel diffusion model employed (top: single direction DTI, bottom: multi direction CSD). Probabilistic thresholds for global approaches are obtained from Figure 8 and are set to the $74^{\text {th }}$ percentile. All paths are reconstructed using FA 0.1 and 90 degrees as thresholds. Darker color indicates the selected cortical ROI, while brighter color indicates those patches which are connected to each other. 


\section{Chapter 3}

\section{Comparative analysis of the macroscale structural connectivity in the macaque and human brain}

Corresponding publication:

Goulas, A.*, Bastiani, M.*, Bezgin, G., Uylings, H.B.M., Roebroeck, A. and Stiers, P. (2014). Comparative Analysis of the Macroscale Structural Connectivity in the Macaque and Human Brain. PLoS Comput Biol 10(3): e1003529. doi: 10.1371/journal.pcbi.1003529

* Authors contributed equally to this work 


\section{Abstract}

The macaque brain serves as a model for the human brain, but its suitability is challenged by unique human features, including connectivity reconfigurations, which emerged during primate evolution. We perform a quantitative comparative analysis of the whole brain macroscale structural connectivity of the two species. Our findings suggest that the human and macaque brain as a whole are similarly wired. A region-wise analysis reveals many interspecies similarities of connectivity patterns, but also lack thereof, primarily involving cingulate regions. We unravel a common structural backbone in both species involving a highly overlapping set of regions. This structural backbone, important for mediating information across the brain, seems to constitute a feature of the primate brain persevering evolution. Our findings illustrate novel evolutionary aspects at the macroscale connectivity level and offer a quantitative translational bridge between macaque and human research. 


\section{Introduction}

Over a century of research has revealed that the brain is inhomogeneous and can be divided based on functional, macro- and micro- structural criteria (Bonin and Bailey, 1947; Brodmann, 1909; Campbell, 1905; Kotter and Wanke, 2005). The regions resulting from such a division are linked through fiber bundles that constitute the neural substrate for the exchange of information between the regions (Schmahmann and Pandya, 2006). Early investigators highlighted the importance of the structural connections of a region to its functions, thus establishing the ground of structure-function dependencies and pinpointing the importance of brain connectivity for fundamental and clinical research (Campbell, 1905; ffytche and Catani, 2005). In recent years, studies offered evidence for the close relation of structural connectivity and function in the mammalian brain (Burns and Young, 2000; Passingham et al., 2002; Saygin et al., 2012). Hence, regions with similar connectivity might be involved in similar functions, and large scale connectivity constitutes a guide to cognition (Bressler and Menon, 2010).

Due to ethical and methodological reasons our most detailed knowledge of the brain originates from animal research. Specifically, the macaque brain serves as a model for the human brain, but such extrapolations might be inaccurate due to rewiring and/or expansion during primate evolution (Krubitzer and Kaas, 2005; Semendeferi et al., 2002; Van Essen and Dierker, 2007) masking out unique features of the human brain (Preuss, 2000). This has important consequences for translating macaque research to humans, which is valuable for cognitive, systems and clinical neuroscience. Hence, there is the need for examining if classical homology criteria such as similarity of connectivity patterns (Campbell and Hodos, 1970; Passingham, 2009) are satisfied.

Diffusion weighted magnetic resonance imaging (dMRI) is used for the examination of the structural connectivity of the brain in vivo and for comparing the structural connectivity of the human and 
macaque brain (Croxson et al., 2005; Frey et al., 2008; Thiebaut de Schotten et al., 2012). However, up to date studies focus on a small subset of brain regions, examining particular fasciculi or lack direct quantitative interspecies comparisons. Hence, interspecies similarities and discrepancies of connectivity patterns and topological features at the whole brain level remain largely concealed.

In addition, dMRI is used for constructing in vivo the whole brain "wiring diagram" of humans, i.e. the human connectome (Sporns et al., 2005). Connectome analysis treats the brain as a complex network and employs tools from network science for unravelling key properties that are pivotal for its proper function and uncovering topological alterations related to mental disorders (Bastiani et al., 2012; Hagmann et al., 2008; van den Heuvel et al., 2010; van den Heuvel and Sporns, 2011). Recent work highlights key properties of the macroscale connectivity of the macaque brain (Modha and Singh, 2010) hinting at potential differences and similarities between the "connectome properties" of the two species, but with no explicit quantitative comparisons taking place.

To complement and surpass limitations of previous comparative studies, we perform a direct comparative quantitative analysis of the macroscale connectional architecture of the macaque and human brain. We adopt a macroscale parcellation scheme called the Regional Map (RM; Bezgin et al., 2012; Kotter and Wanke, 2005) and we construct whole brain species-specific connectomes, with the aid of dMRI for the human and a neuroinformatics database for the macaque brain. Subsequently, we quantify the similarity of connectivity patterns, global topological features, and topology of the brain regions of the two species. This approach succeeds in uncovering preserved and divergent features of the macroscale connectional architecture of the brain of these two primate species. 


\section{Materials and Methods}

Whole brain parcellation scheme

For the whole brain examination of both species we employed a map specifically designed for this purpose, the RM (Bezgin et al., 2012; Kotter and Wanke, 2005) (Fig. 1A). This map consists of putative homologues between the two species based on structural, macroscopic and functional criteria. Its level of coarseness is dictated from the size of regions that are discernible in both human and macaque brains (Kotter and Wanke, 2005). No connectivity criteria were used for the delineation of the various regions constituting the RM. The RM was delineated on the F99 standard brain which is based on an MRI scan of one macaque brain. Subsequently, the RM was morphed to match the human brain by using macroscopic and functional landmarks (Orban et al., 2004; Van Essen, 2004). In total 82 regions (41 for each hemisphere) constituted the RM that we used (Table S1). We should note that the use of the RM is necessitated by the lack of an unequivocal "standard" microstructure based map for even the brain of one species, let alone a "standard" microstructure based comparative map (Van Essen et al., 2012a). Moreover, the regions constituting the RM are larger than regions defined based on e.g. cytoarchitecture, the so-called cortical fields, and one RM region might include various such cortical fields. This level of granularity of the RM was deliberately chosen in order to circumvent controversial issues with respect to macaque-human cortical field homologies across the whole brain, like the presence of more cortical fields in the human brain and/or duplication of certain cortical fields (Kotter and Wanke, 2005; Sereno and Tootell, 2005; Van Essen et al., 2012a; Van Essen et al., 2012b). 
A
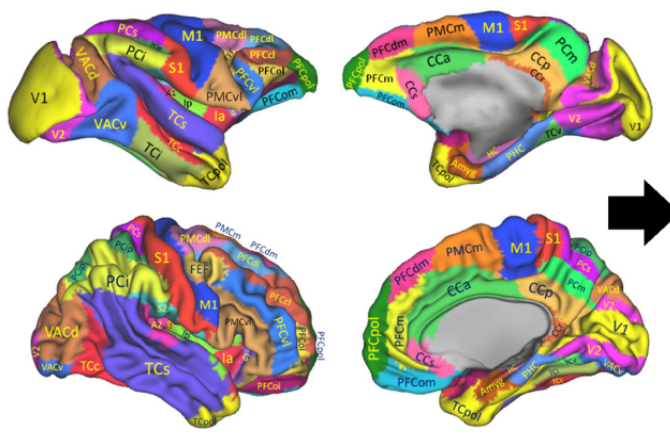

C

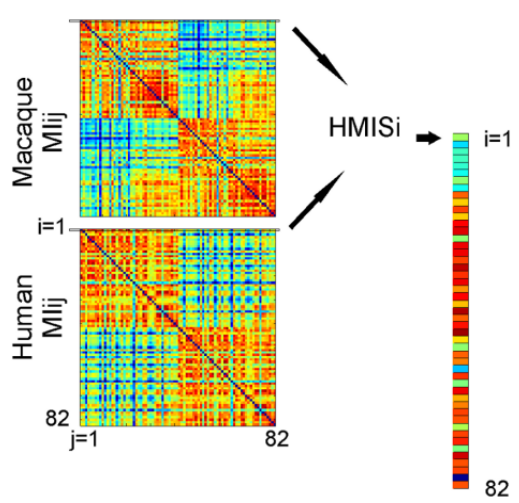

B
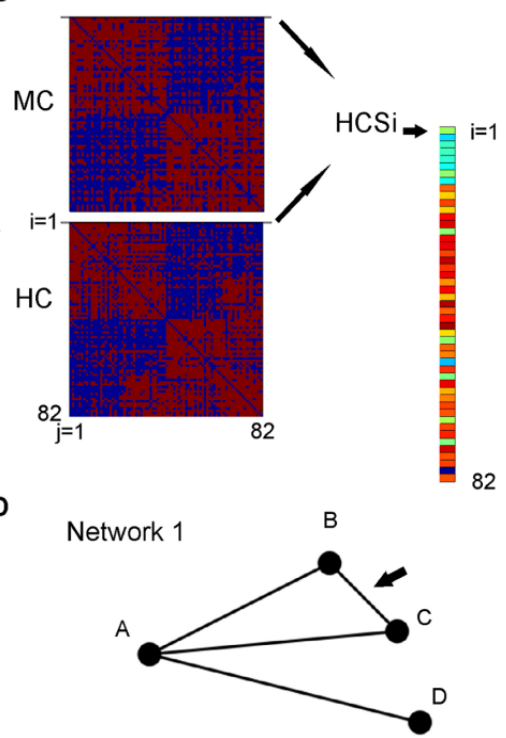

Network 2

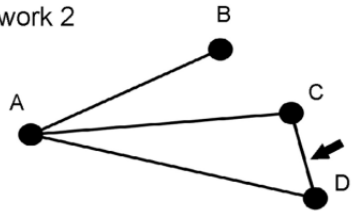

Figure 1: Schematic depiction of the main points of the analysis. A) The RM was used in order to construct the connectivity matrices of the two species, i.e. the MC and HC (panel B). The resulting connectivity matrices have $i=82$ rows and $j=82$ columns. B) Example of the calculation of the HCS for region $i=1$. The entries of row 1 from the MC

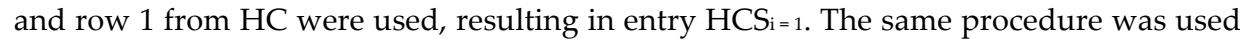
for all regions, resulting in a $1 \times 82$ vector HCS containing the HCS indexes of all the regions. C) Example of calculation of the HMIS for region $\mathrm{i}=1$. The $\mathrm{MC}$ and $\mathrm{HC}$ were used for the separate calculation of the matching index matrices $\mathrm{MI}_{\mathrm{ij}}$ (one for each species separately). The HMIS index for region $i=1$ is calculated from the entries of row 1 of the matching index matrices of the two species. The procedure is repeated for all regions, resulting in a $1 \times 82$ vector HMIS containing the HMIS indexes of all the regions. D) Toy networks demonstrating differences of the HCS and the HMIS. Given two hypothetical "brains" that form a network with 4 regions and 4 connections the HCS and HMIS indexes are calculated for brain region A. On the one hand, the HCS index is equal to 1 (perfect similarity), since region $\mathrm{A}$ in both networks is connected exactly to the same regions, i.e. nodes $B, C$ and D. On the other hand, the HMIS index is -0.5 , indicating a divergence of the connectivity similarity profiles of region A in networks/brains 1 and 2 . These discrepancies arise from the "rewiring" of the connection marked with an arrow. 


\section{Macaque whole brain connectome}

We used the RM and the CoCoMac database (http://cocomac.gnode.org) to assemble the whole brain connectome of the macaque. The database was accessed on December 2010. Briefly, the CoCoMac database consists of entries describing the presence of a structural connection between two regions, as revealed by tracing studies, and have the format: region $\mathrm{A}$ has an efferent connection with region $\mathrm{B}$. The database contains over 400 studies spanning several decades and thus represents a current best estimate of the macroscale connectivity of the macaque brain. Different researchers use different maps with divergent nomenclature. In order to link the different maps the database contains relation codes with the format: Region A of map X is identical to region $B$ of map Y. Dedicated algorithms and algebra is used to map regions of one map to regions of a "reference" map (Bezgin et al., 2008; Stephan et al., 2000). In the current study, the RM functioned as the "reference" map and thus available connectivity information contained in the database was represented as an $\mathrm{NxN}$ connectivity matrix, where $\mathrm{N}=82$ the regions constituting the RM. A non-zero matrix entry Aij denotes the presence of a connection from region $i$ and $j$. In order to compare the connectivity of the macaque and human brain (see below), and since directionality of structural connections cannot be inferred in vivo in the human brain, the directed connectivity matrix of the macaque was symmetrized and binarized by taking into account all connections irrespective of their strength. The resulting macaque connectome (MC) consisted of 1857 undirected connections/edges between 82 regions/nodes. The binarization step is necessary since the connectomes of the two species were assembled from different modalities. DMRI and tractography is not adequate for inferring density of connections (Jones et al., 2013) contrary to invasive tracing techniques in monkey studies. This limitation and the fact that certain network metrics employed for cross-species comparisons involve cross-matrix operations (see below), 
do not allow the use of a weighted approach, since the weights obtained from the different modalities are not comparable (see also Discussion).

Human whole brain connectome

Data acquisition

Whole brain scans of five healthy subjects (2 females, age mean: 29.4 std: 3.2) were acquired after obtaining written informed consent. Data acquisition and preprocessing are described in (Bastiani et al., 2012). Briefly, data were collected using a Siemens 3T MAGNETOM Allegra MR scanner equipped with a high slew-rate head gradient-coil (amplitude $40 \mathrm{mT} / \mathrm{m}$, slew rate $400 \mathrm{~T} / \mathrm{m} / \mathrm{s}$ ) and an 8 -channel phasedarray head RF-coil was used to acquire the data. A double refocused spin-echo diffusion sequence was used to acquire 131 volumes of data, with TR $=6600 \mathrm{~ms}, \mathrm{TE}=94 \mathrm{~ms}$, b-value $=3000 \mathrm{~s} / \mathrm{mm} 2,88 \times 88$ matrix, 52 axial slices, $2.5 \times 2.5 \times 2.5 \mathrm{~mm}$ voxels, partial Fourier $=6 / 8$ and a bandwidth of $2840 \mathrm{~Hz} /$ pixel (echo-spacing $0.4 \mathrm{~ms}$ ). A total of 120 diffusion gradient directions were acquired with 11 unweighted $(b=0 \mathrm{~s} / \mathrm{mm} 2)$ volumes acquired after every 12 gradient directions and including the first and last volumes. A T1-weighted 3D MPRAGE scan (TR $=2250, \mathrm{TE}=2.6 \mathrm{~ms}$, flip angle $=9^{\circ}, 256 \times 256$ matrix, 192 sagittal slices, $1 \times 1 \times 1 \mathrm{~mm}$ voxels) was acquired for gray/white matter boundary segmentation.

Voxel-wise diffusion model estimation

A multi-direction high angular resolution diffusion imaging based model has been used to estimate the voxel-wise orientation of neuronal fibers. Constrained spherical deconvolution fiber orientation distributions were reconstructed (Tournier et al., 2007) over a five-fold tessellated icosahedron. This technique was selected for its robustness in estimating orientational distributions from high angular resolution diffusion imaging data. Moreover, fiber orientation distributions represent actual fiber orientation distributions rather than water-bound spin displacements, which leads to stable and accurate local orientations 
that are very beneficial for both local and global tractography purposes (Fillard et al., 2011).

\section{Tractography algorithm and parameters}

A local probabilistic tractography algorithm was used in this study. The employed algorithm uses orientations sampled from the fiber orientation distributions at each step and initializes a great number of streamlines per seed point in a way similar to the PICo algorithm (Parker et al., 2003). This tractography algorithm has shown good performance based on empirical data (Bastiani et al., 2012) and phantom based evaluations (Li et al., 2012). Per seed point, 3000 streamlines were initiated within a sphere whose center corresponded to the center of every white matter boundary voxel and whose radius has been set to half the voxel size $(1.25 \mathrm{~mm})$. The step size was set to $1 \mathrm{~mm}$ and the angular thresholds to $30^{\circ}$.

Fractional anisotropy maps were thresholded at a value of 0.1 in order to obtain the white matter waypoint masks. These are binary masks containing only those voxels where fibers are allowed to propagate. The tractography algorithm used in the present study was run in original diffusion data space. Therefore, we have chosen to use median filtered fractional anisotropy masks computed in that same space, instead of white matter masks obtained from T1-weighted volumes segmentation, in order to achieve maximum integrity and alignment of white matter masks to the diffusion data. To avoid influences on fractional anisotropy such as partial volume effects at the white/grey matter boundary and in those voxels where more than two diffusion directions are reconstructed a 3-dimensional median filter has been applied to the thresholded white matter volumes to fill holes in the masks. Furthermore, in the white matter binary masks, white matter boundary voxels were always included in the volume after having thresholded the fractional anisotropy mask and used the median filter. Fibers shorter than $10 \mathrm{~mm}$ or longer than $200 \mathrm{~mm}$ were removed. Moreover, looping fibers (i.e. fibers that return to already explored 
voxels) are excluded from the analysis. Probabilistic local multidirection tractography was performed using the MRtrix package (Tournier et al., 2007). To move from a very high resolution tractography result that connects all $\sim 30000$ voxels in the white matter bound voxel set to the weighted connectivity matrix based on the 82 regions of the RM, we employed the same connectivity index as defined in (Iturria-Medina et al., 2011). This index of connectivity between two regions is given by the sum of the weights connecting all the voxels between region A and region B and vice versa, normalized by the sum of the number of seeds used in each region. Thus, any non-zero weight connecting any voxel in one parcel to any voxel in another in either of the two directions connects the two parcels in the final symmetric adjacency matrix (Iturria-Medina et al., 2011). Such step aims at reconstructing the weighted $82 \times 82$ connectivity matrix eliminating the effect of patch-area normalization. To create the human connectome (HC) the symmetrized weighted matrices obtained from each individual were averaged. Subsequently, a certain threshold has to be applied to the probabilistic tractography results. Thresholding constitutes a necessary step in connectome reconstruction (Hagmann et al., 2008; van den Heuvel and Sporns, 2011). No thresholding option provides a definite answer about the "true" underlying connections for the whole brain. Consequently, the threshold was chosen in a way not to uncover the "true" connections, but to render the MC and HC comparable, which serves the purpose of the current study. Hence, only the highest weights were selected and their number was chosen to match the number of connections of the MC. Finally, the HC was binarized. Hence, the MC and HC are binary undirected matrices with the same number of nodes and connections, corresponding to a density of 0.559 . It should be noted that the vast majority of the connections (85\%) in the average matrix were present in at least $4 / 5$ of the individual matrices (after thresholding at the same density). Therefore, averaging across the participants did not bias towards connections that have particularly high weight in a minority of the subjects. A very good interindividual 
similarity was also observed with a high correlation between the unthresholded weighted matrices of each subject (mean: 0.86 range: 0.83-0.90).

\section{Comparing the macaque and human connectome}

We aimed at examining key topological properties of the MC and HC. Below we introduce the various network metrics, defined at a regionwise or whole brain level, with relevant references.

Given two matrices $\mathrm{A}$ and $\mathrm{B}$, representing the $\mathrm{MC}$ and $\mathrm{HC}$ respectively, we computed the intersection network $X$ defined as:

$$
X=A \cap B
$$

The number of edges $\mathrm{Lx}$ of the intersection network denotes the common edges/connections of the MC and HC. Lx divided by the total number of edges $\mathrm{L}(=1857)$ in each network A and B offers a measure of similarity of the two networks. Hence, the ratio $\mathrm{L}_{x} / \mathrm{L}$ ranges from 0 to 1 , indicating no overlap and complete overlap respectively of the edges of networks $\mathrm{A}$ and $\mathrm{B}$.

We next proceeded to a region-wise analysis and sought to quantify the overlap of the connections of the assumed homologues of the MC and HC. This was performed with the Homologue Connectivity Similarity (HCS) metric:

$$
H_{C S}(A, B)=\frac{\left|\Gamma_{i}(A) \bigcap \Gamma_{i}(B)\right|}{\left|\Gamma_{i}(A) \bigcup \Gamma_{i}(B)\right|}
$$

The interspecies overlap/intersection of connections of region $\mathrm{i}$ in the macaque and human brain, represented by A and B respectively, is denoted by $\left|\Gamma_{i}(A) \cap \Gamma_{i}(B)\right|$ and the union with $\left|\Gamma_{i}(A) \cup \Gamma_{i}(B)\right|_{\text {with }}$ 
$\Gamma_{i}$ denoting the set of neighbors of node $\mathrm{i}$ and $|\cdot|$ the size of the set (see Fig. $1 \mathrm{~B}$ ). Hence, for each region $\mathrm{i}=1 \ldots 82$ of the RM the HCS $\mathrm{H}_{\mathrm{i}}$ ranges from 0 to 1 and indicates respectively low and high interspecies connectivity similarity of the assumed homologue region $i$.

Subsequently, we quantified a "second-order" similarity of homologous regions, i.e. their connectivity similarity profile with the rest of the brain. To this end, we used the matching index (Hilgetag et al., 2002) for A, denoting MC (the same for B denoting the HC):

$$
M I_{i j}(A)=\frac{\left|\Gamma_{i}(A) \cap \Gamma_{j}(A)\right|}{\left|\Gamma_{i}(A) \cup \Gamma_{j}(A)\right|}
$$

This resulted in one MI matrix for each species, with the row i capturing the connectivity similarity of region i with all the other brain regions of the same species. Hence, each row of each MI matrix can be conceived as a "connectivity similarity profile" of a region constructed for each species separately. In order to quantify if the connectivity similarity profile of putative homologous regions was preserved, we calculated the correlation between row $\mathrm{i}=1 \ldots 82$ of the MI matrices (without including the diagonal entries of $\mathrm{MI}_{\mathrm{A}}, \mathrm{MI}_{\mathrm{B}}$, i.e no self-similarity values). This resulted in the Homologue Matching Index Similarity (HMIS):

$$
H M I S_{i}\left(M I_{A}, M I_{B}\right)=r\left(M I_{A i}, M I_{B i}\right)
$$

with $\mathrm{r}$ denoting Pearson's correlation coefficient of row i of the two MI matrices (Fig. 1C).

Main aspects of the topology of the RM regions in the whole brain network, i.e. centrality and clustering, quantifying "importance" and "segregation" of regions (Sporns et al., 2007), were examined by calculating the betweenness centrality (BC; Freeman, 1978), eigenvector 
centrality (EC; Bonacich, 1972) and clustering coefficient (C; Watts and Strogatz, 1998). Segregation in this context implies a tightly interconnected neighborhood of a brain region, allowing "cross-talk" and exchange of information, while "importance" refers to highly central regions that are topologically ideal for information integration (Rubinov and Sporns, 2010). All these metrics were calculated for each species separately by using the formulas for binary undirected networks described in (Rubinov and Sporns, 2010; Zuo et al., 2012). In order to assess perseverance of the centrality and clustering of the brain of the two species, Spearman's rank correlation between the same network metrics from the two species was computed. A statistically significant (positive) correlation would indicate the evolutionary perseverance of each network metric.

Lastly we examined the presence of a rich club organization, indicating the presence of a "structural backbone", quantified with the rich club coefficient (RCC; Colizza et al., 2006):

$$
\phi(k)=\frac{2 E_{>k}}{N_{>k}\left(N_{>k}-1\right)}
$$

where $\mathrm{E}_{>\mathrm{k}}$ denotes the number of connections/edges that exist among nodes/regions that exhibit more connections than a given number $\mathrm{k}$ and $\mathrm{N} \gg \mathrm{k}$ denotes the number of nodes/regions that have degree higher than $k$, i.e. exhibit more connections than a given number $k$. The RCC is calculated for a range of $\mathrm{k}$ for a given network and for a number of randomized matched networks in order to estimate the RCC values expected by chance. This results in a normalized RCC:

$$
\phi_{\text {norm }}(k)=\frac{\phi(k)}{\phi_{\text {rand }}(k)}
$$


Values higher than 1 for a range of values $\mathrm{k}$ indicate that the network is characterized by a rich club structure, with nodes with degree higher than $\mathrm{k}$ linked with more connections than expected by chance (Colizza et al., 2006).

For each analysis, 10000 random networks, unless otherwise stated, matching each MC and HC in number of nodes, edges and degree distribution were created, with the use of a degree preserving algorithm (Rao et al., 1996). The random networks were used for calculating p-values and z-scores of the network metrics. Thus, the random networks are used for obtaining "null" values for the metrics used. For the region-wise analysis, introducing multiple tests, we used a conservative Bonferroni correction.

For verifying the robustness of the findings of the topology of the original $\mathrm{MC}$ and $\mathrm{HC}$, we performed the following control analyses. First, we perturbed the MC and $\mathrm{HC}$ by rewiring the network with a low probability, i.e. 0.1 , while keeping the number of edges, nodes and degree distribution intact. That is, a pair of edges was swapped with 0.1 probability, thus introducing modest alterations to the network. Secondly, connections in empty positions were randomly and uniformly inserted. We inserted $10 \%$ of the initial number of connections, i.e. $1857^{*} 10 \%=186$ connections. Random networks for addressing the significance of the results in these "randomly enriched" MC and HC matched the new higher density. This type of analysis simulates in a simple way a scenario were previously absent connections appear to be present (Markov et al., 2011). Techniques like the aforementioned ones were used for the examination of the robustness of features of the HC (Hagmann et al., 2008). Additionally, we examined the effect of the choice of a particular parcellation by performing all the analyses on the connectomes in the exact same way, but assembled based on a different parcellation scheme (Brodmann, 1909). Lastly, due to limitations of dMRI-based local probabilistic tractography techniques in revealing long controlateral connections (Bastiani et al., 2012; Li et al., 2012) and 
the lack of complete information on such connections in the CoCoMac database, we performed a within hemisphere analysis for the left and right hemisphere separately. To this end, we constructed the MC and $\mathrm{HC}$ as previously described but for each hemisphere separately. The hemisphere-wise MC appeared very dense (left hemisphere: 0.791 right hemisphere: 0.792 density, compared to 0.559 for the whole brain connectome) and the $\mathrm{HC}$ was thresholded accordingly. The very high density of the hemisphere-wise connectomes poses a problem for a binary analysis. This is due to the fact that many topological properties of the original $\mathrm{MC}$ and $\mathrm{HC}$ will not differ from their rewired counterparts because of inefficient "space" for rewiring. Taking an illustrative case for example, the rich club analysis will reveal dense connections between regions at increased levels of $\mathrm{k}$ but such strong interconnectivity can be merely explained by the very high density of the network and is not therefore not "surprising"/statistically significant (for a similar discussion but a different direction see: Ercsey-Ravasz et al., 2013). Therefore, we decided to adopt weighted networks and suitable weighted versions of the aforementioned metrics. As previously explained, the weights from the $\mathrm{MC}$ and $\mathrm{HC}$ are not comparable. Therefore, we restricted the weighted hemisphere-wise analysis to metrics that do not involve cross-matrix operations (thus the intersection and HCS were not computed). The weighted version of the HMIS involved equation (4) but operating on the rows of a "weighted matching index" matrix obtained as the cosine similarity between rows $i$ and $\mathrm{j}$ of matrix $\mathrm{A}$, denoting $\mathrm{MC}$ (the same for $\mathrm{B}$ denoting the $\mathrm{HC}$ ):

$$
M I_{i j}^{\text {weighted }}(A)=\frac{A_{i} \cdot A_{j}}{\left\|A_{i}\right\|\left\|A_{j}\right\|}
$$

The weighted version of EC, BC and C was computed as described in (Rubinov and Sporns, 2010). The weighted rich club can be formulated as follows (Opsahl et al., 2008): 


$$
\phi_{\text {weighted }}(k)=\frac{W_{>k}}{\sum_{i=1}^{E_{>k}} W_{i}^{\text {ranked }}}
$$

with $W_{>k}$ denoting the sum of the weights of the edges connecting nodes with degree higher than $\mathrm{k}$ and $\sum_{i=1}^{E_{>k}} W_{i}^{\text {ranked }}$ denoting the sum of the $\mathrm{E}>\mathrm{k}$ first ranked (in decreasing order) edge weights in the whole network. For each analysis, 10000 random networks (corresponding to the "link and weight reshuffle" model in: Opsahl et al., 2008) matching each hemisphere-wise MC and HC in number of nodes, edges and degree distribution were created, with the use of a degree preserving algorithm (Rao et al., 1996).

All network analysis was performed with the use of functions from the Brain Connectivity Toolbox (https://sites.google.com/site/bctnet/) (Rubinov and Sporns, 2010) and custom scripts written in Matlab (Mathworks). The MATLAB code for the computation of the HCS is provided (Software S1). Brain renderings were performed with the following freely available software: Caret (http://brainvis.wustl.edu/wiki/index.php/Caret:About) and BrainGL (http://code.google.com/p/braingl/). For certain visualizations of the connections of the MC and HC a mean-shift edge bundling algorithm was used (Bottger et al., 2014).

\section{Results}

Fig. 2 depicts the MC and $\mathrm{HC}$ and their intersection. Their intersection, i.e. the $\mathrm{L}_{\mathrm{x}} / \mathrm{L}$ ratio of the $\mathrm{MC}$ and $\mathrm{HC}$ was significant $\left(\mathrm{L}_{\mathrm{x}} / \mathrm{L}_{\text {original }}=0.754 \mathrm{p}<\right.$ $0.001, \mathrm{~L}_{x} / \mathrm{Lnull}$ mean $=0.599 \mathrm{std}=0.006$, null values from 1000 random networks). Thus, the wiring of the macaque and human brain as a whole is more similar than expected by chance. 

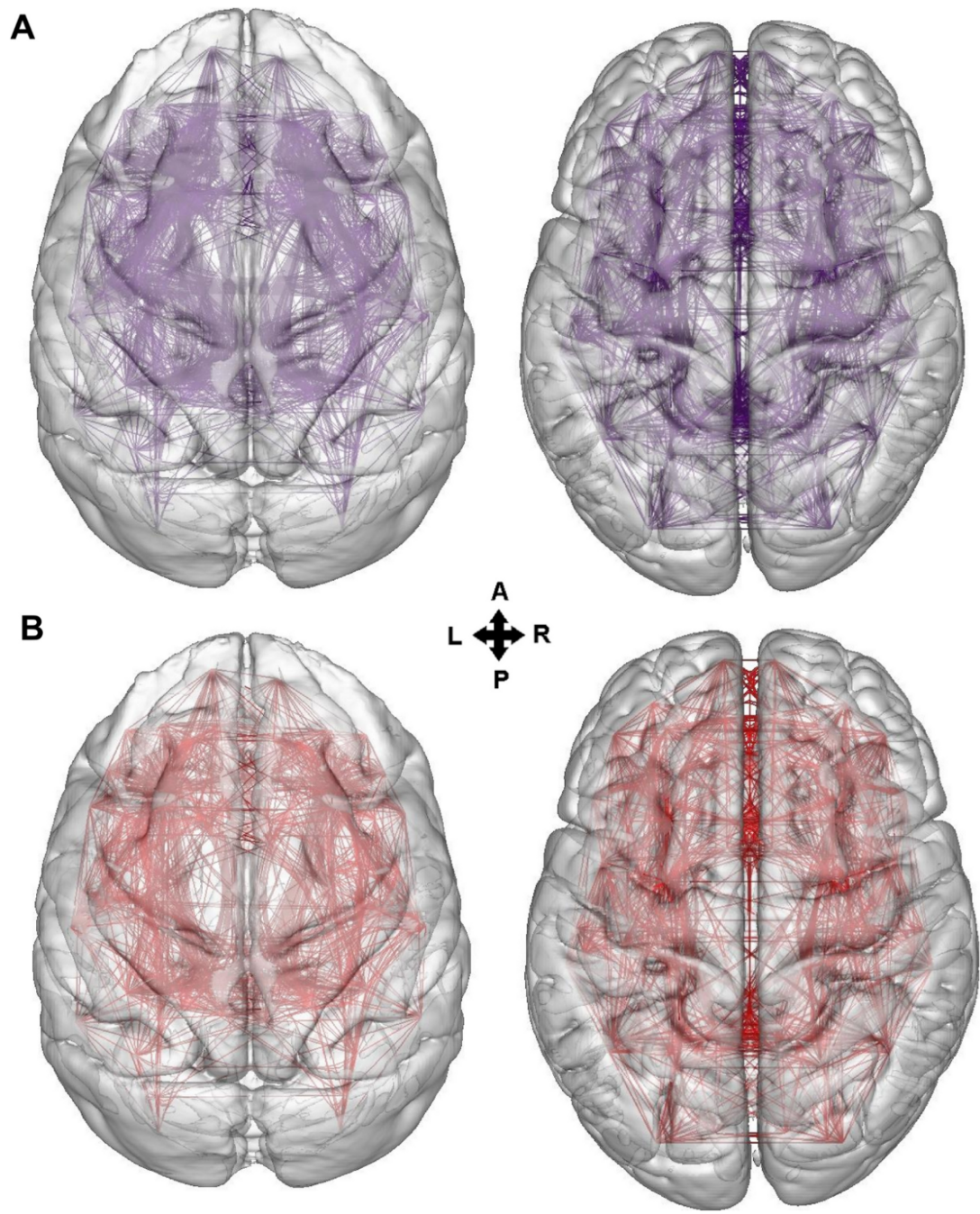

Figure 2: Overlap of the MC and HC. A) Rendering of the MC and HC. B) Rendering of the overlap between the MC and HC. The two connectomes exhibit a statistically significant overlap (see Results). The renderings were performed with BrainGL and a mean-shift edge bundling algorithm (Bottger et al., 2014).

The region-wise analysis of the HCS revealed significant connectivity preservation for many RM regions. Specifically, a set of frontal, occipital and temporal regions exhibited significant preservation of their whole brain connectivity across the species. Mainly parietal and 
cingulate regions appeared to lack such preservation (Fig. 3A, Table 1). In sum, 51 out of 82 RM regions exhibited significant HCS and therefore communicate with a significantly overlapping set of brain regions in both species.

\begin{tabular}{|c|c|c|c|c|c|c|c|c|}
\hline \multirow[t]{3}{*}{ RM acronyms } & \multicolumn{4}{|c|}{ Right } & \multicolumn{4}{|c|}{ Left } \\
\hline & \multicolumn{2}{|c|}{ HCS } & \multicolumn{2}{|c|}{ HMIS } & \multicolumn{2}{|c|}{ HCS } & \multicolumn{2}{|c|}{ HMIS } \\
\hline & z-score & p-value & z-score & p-value & z-score & p-value & z-score & p-value \\
\hline PFCoi & 3.08 & 0.0015 & 2.94 & 0.0014 & 3.31 & 0.0007 & 2.61 & 0.0042 \\
\hline PFCom & 3.39 & 0.0003 & 3.57 & 0.0001 & 2.41 & 0.0135 & 5.08 & 0.0001 \\
\hline PFCol & 3.77 & 0.0002 & 6.29 & 0.0001 & 3.49 & 0.0006 & 6.85 & 0.0001 \\
\hline G & 2.62 & 0.0046 & 3.77 & 0.0001 & 3.65 & 0.0002 & 5.45 & 0.0001 \\
\hline PFCpol & 2.90 & 0.0040 & 3.30 & 0.0006 & 3.62 & 0.0002 & 5.15 & 0.0001 \\
\hline PFCvl & 3.91 & 0.0001 & 4.19 & 0.0001 & 4.73 & 0.0001 & 3.79 & 0.0001 \\
\hline $\mathrm{PFCm}$ & 4.35 & 0.0001 & 3.11 & 0.0007 & 3.73 & 0.0001 & 2.10 & 0.0170 \\
\hline PFCcl & 4.86 & 0.0001 & 5.09 & 0.0001 & 4.28 & 0.0001 & 4.85 & 0.0001 \\
\hline M1 & 3.67 & 0.0004 & 3.86 & 0.0001 & 3.84 & 0.0003 & 3.91 & 0.0001 \\
\hline PFCdm & 2.04 & 0.0360 & 1.07 & 0.1390 & 2.12 & 0.0310 & 3.36 & 0.0001 \\
\hline FEF & 3.37 & 0.0006 & 3.43 & 0.0001 & 2.59 & 0.0071 & 3.75 & 0.0001 \\
\hline PFCdl & 3.72 & 0.0003 & 3.82 & 0.0001 & 4.26 & 0.0001 & 3.14 & 0.0004 \\
\hline PMCVl & 3.82 & 0.0001 & 5.62 & 0.0001 & 4.35 & 0.0001 & 5.76 & 0.0001 \\
\hline $\mathrm{PMCm}$ & 3.80 & 0.0003 & 2.57 & 0.0040 & 4.04 & 0.0001 & 2.64 & 0.0036 \\
\hline PMCdl & 4.26 & 0.0001 & 3.42 & 0.0001 & 5.30 & 0.0001 & 3.16 & 0.0007 \\
\hline Tcpol & 3.18 & 0.0012 & 2.47 & 0.0066 & 3.66 & 0.0001 & 2.11 & 0.0166 \\
\hline TCs & 4.29 & 0.0001 & 4.13 & 0.0001 & 4.26 & 0.0001 & 4.37 & 0.0001 \\
\hline TCC & 5.13 & 0.0001 & 4.68 & 0.0001 & 4.45 & 0.0001 & 4.51 & 0.0001 \\
\hline $\mathrm{TCi}$ & 4.01 & 0.0001 & 6.14 & 0.0001 & 4.41 & 0.0001 & 5.61 & 0.0001 \\
\hline TCv & 3.33 & 0.0005 & 1.94 & 0.0242 & 3.12 & 0.0010 & 1.87 & 0.0291 \\
\hline A1 & 3.07 & 0.0015 & 2.58 & 0.0036 & 3.53 & 0.0006 & 3.26 & 0.0002 \\
\hline A2 & 2.89 & 0.0039 & 2.16 & 0.0137 & 3.66 & 0.0003 & 3.34 & 0.0001 \\
\hline S1 & 3.94 & 0.0001 & 2.57 & 0.0046 & 3.33 & 0.0009 & 3.27 & 0.0004 \\
\hline S2 & 4.11 & 0.0001 & 3.20 & 0.0007 & 4.05 & 0.0001 & 3.07 & 0.0006 \\
\hline $\mathrm{PCi}$ & 4.21 & 0.0001 & 0.01 & 0.5032 & 4.03 & 0.0001 & 0.48 & 0.3214 \\
\hline $\mathrm{PCm}$ & 2.63 & 0.0089 & 2.17 & 0.0136 & 2.91 & 0.0058 & 2.49 & 0.0052 \\
\hline PCip & 3.67 & 0.0003 & 0.54 & 0.2936 & 3.24 & 0.0019 & 0.87 & 0.1910 \\
\hline PCs & 1.96 & 0.0441 & 1.90 & 0.0284 & 1.54 & 0.1036 & 1.94 & 0.0253 \\
\hline V1 & 3.52 & 0.0001 & 3.39 & 0.0003 & 3.55 & 0.0002 & 4.48 & 0.0001 \\
\hline V2 & 3.47 & 0.0007 & 4.10 & 0.0001 & 4.09 & 0.0001 & 4.03 & 0.0001 \\
\hline VACV & 4.27 & 0.0001 & 5.67 & 0.0001 & 4.32 & 0.0001 & 4.92 & 0.0001 \\
\hline VACd & 4.32 & 0.0001 & 4.22 & 0.0001 & 3.94 & 0.0001 & 4.44 & 0.0001 \\
\hline Amyg & 4.92 & 0.0001 & 4.99 & 0.0001 & 4.86 & 0.0001 & 5.60 & 0.0001 \\
\hline $\mathrm{PHC}$ & 3.96 & 0.0001 & 1.80 & 0.0337 & 2.74 & 0.0058 & 1.70 & 0.0432 \\
\hline $\mathrm{HC}$ & 2.92 & 0.0025 & 3.64 & 0.0002 & 2.73 & 0.0033 & 3.11 & 0.0009 \\
\hline CCs & 2.38 & 0.0160 & 0.65 & 0.2650 & 2.53 & 0.0102 & 0.05 & 0.4836 \\
\hline $\mathrm{CCr}$ & 2.14 & 0.0239 & 0.12 & 0.4633 & 2.43 & 0.0122 & -0.22 & 0.5884 \\
\hline CСp & 3.26 & 0.0009 & 1.46 & 0.0727 & 3.90 & 0.0001 & 2.09 & 0.0164 \\
\hline $\mathrm{CCa}$ & 2.27 & 0.0271 & -0.79 & 0.7836 & 1.84 & 0.0646 & -0.33 & 0.6341 \\
\hline Ia & 4.65 & 0.0001 & 4.47 & 0.0001 & 4.01 & 0.0001 & 4.78 & 0.0001 \\
\hline Ip & 1.34 & 0.1502 & 0.21 & 0.4200 & 1.18 & 0.1851 & -0.03 & 0.5145 \\
\hline
\end{tabular}

Table 1: HCS and HMIS results for the RM regions for whole brain binary network analysis. Regions exhibiting significant HCS and HMIS values $(\mathrm{p}<0.05$ Bonferroni corrected) are in bold. P-values and z-scores are also depicted. 


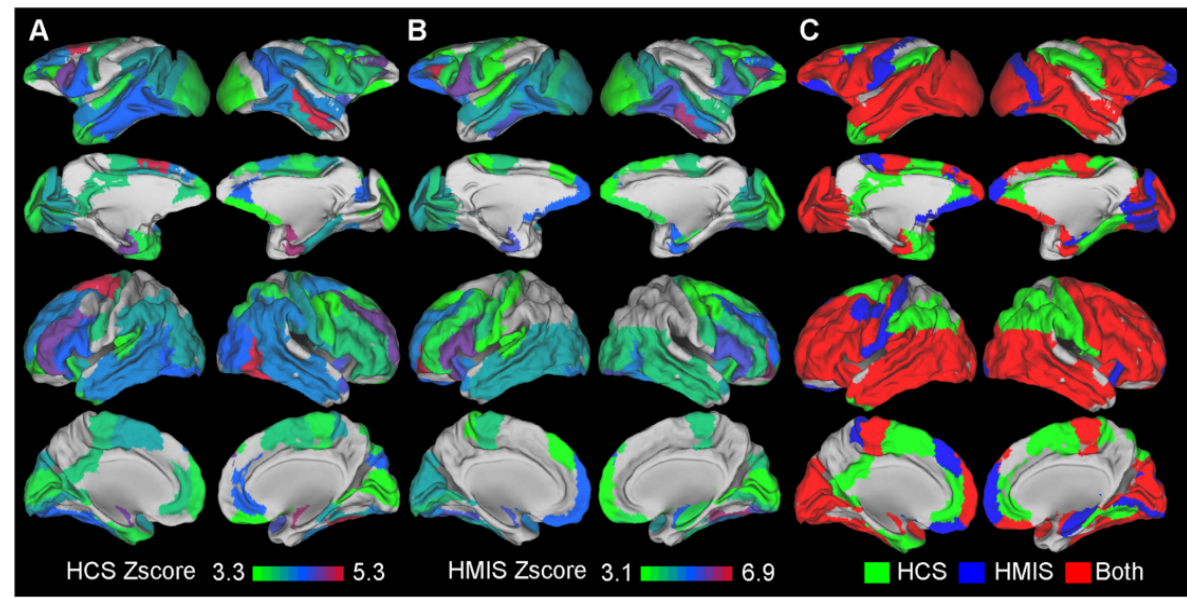

Figure 3: Renderings of the macaque and human regions exhibiting a significant HCS and HMIS index. A) HCS index B) HMIS index. In both panels only regions reaching significance are depicted ( $\mathrm{p}<0.05$ Bonferroni corrected). Color coding denotes their corresponding z-score. C) Summary of results by colour coding the regions based on the preservation of both HMIS and HCS (red), only HCS (green), only HMIS (blue).

The region-wise analysis of the HMIS revealed that 45 out of 82 RM regions, mainly involving frontal, temporal, occipital regions reached significance. Cingulate and parietal regions failed to reach significance (Fig. 3B, Table 1). Hence, regions reaching significance seem to form the same "connectivity coalitions", i.e. exhibit a statistically significant connectivity similarity profile with the rest of the brain regions in macaques and humans. This in turn can entail that their "functional coalitions" might also be preserved. Conversely, certain regions fail to reach significance and might suggest that "evolutionary rewiring" occurred in such a way that they formed distinct "connectivity coalitions" with the rest of the brain regions in the two species.

The HCS and HMIS results involve distinct but also overlapping sets of regions (Fig. 3C, Table 1). Thus, they illustrate converging but also diverging aspects of distinct connectional characteristics of the brain regions of the two species. 
The hemisphere-wise weighted HMIS analysis revealed broadly the same pattern with a notable difference: a subset of prefrontal and temporal regions did not reach significance (Table S2). Notably, even in this type of analysis cingulate regions failed to reach significance.

Fig. 4 depicts the results of the centrality and clustering analysis of the MC and HC. In both species, a general pattern is discernible with regions in "association" cortex exhibiting the highest centrality and regions in "primary" cortex exhibiting the lowest (Fig. 4A and B). Moreover, in both species the cingulate cortex appears as highly central (Fig. 4A and B). Additionally, little overlap was observed between the macaque and the human brain when taking into account the regions that are highly central (centrality $>$ mean +1 std of the centrality of the RM regions) (Table S3). It should be noted however, that bilateral posterior cingulate cortex (CCp) and left inferior parietal cortex (PCi) exhibited high ( $>$ the mean +1 std threshold) BC and EC in both species indicating the perseverance of the prominent central role of these regions. However, a region wise correlation of the $\mathrm{BC}$ and $\mathrm{EC}$ values across the species revealed a relative high but not significant correlation $($ rho $=0.51,0.52$ respectively $\mathrm{p}>0.1)$. This might suggest that, at a whole brain level, there is a lack of perseverance of the topological importance of the assumed homologues of the macaque and human brain (see also Fig. S1 A and B for scatter plots of the BC and EC values from the two species).

The C values for both species exhibited a "reversed" pattern with the centrality values: regions in "association" cortex exhibiting the lowest values and regions in "primary" cortex exhibiting the highest (Fig. 3C). The $C$ values across the species did not reach significance either (rho $=0.46 \mathrm{p}>0.1$ ). Therefore, the regions of the brains of the two species seem to exhibit different levels of segregation (see also Fig. S1 C for scatter plots of the $C$ values from the two species). 


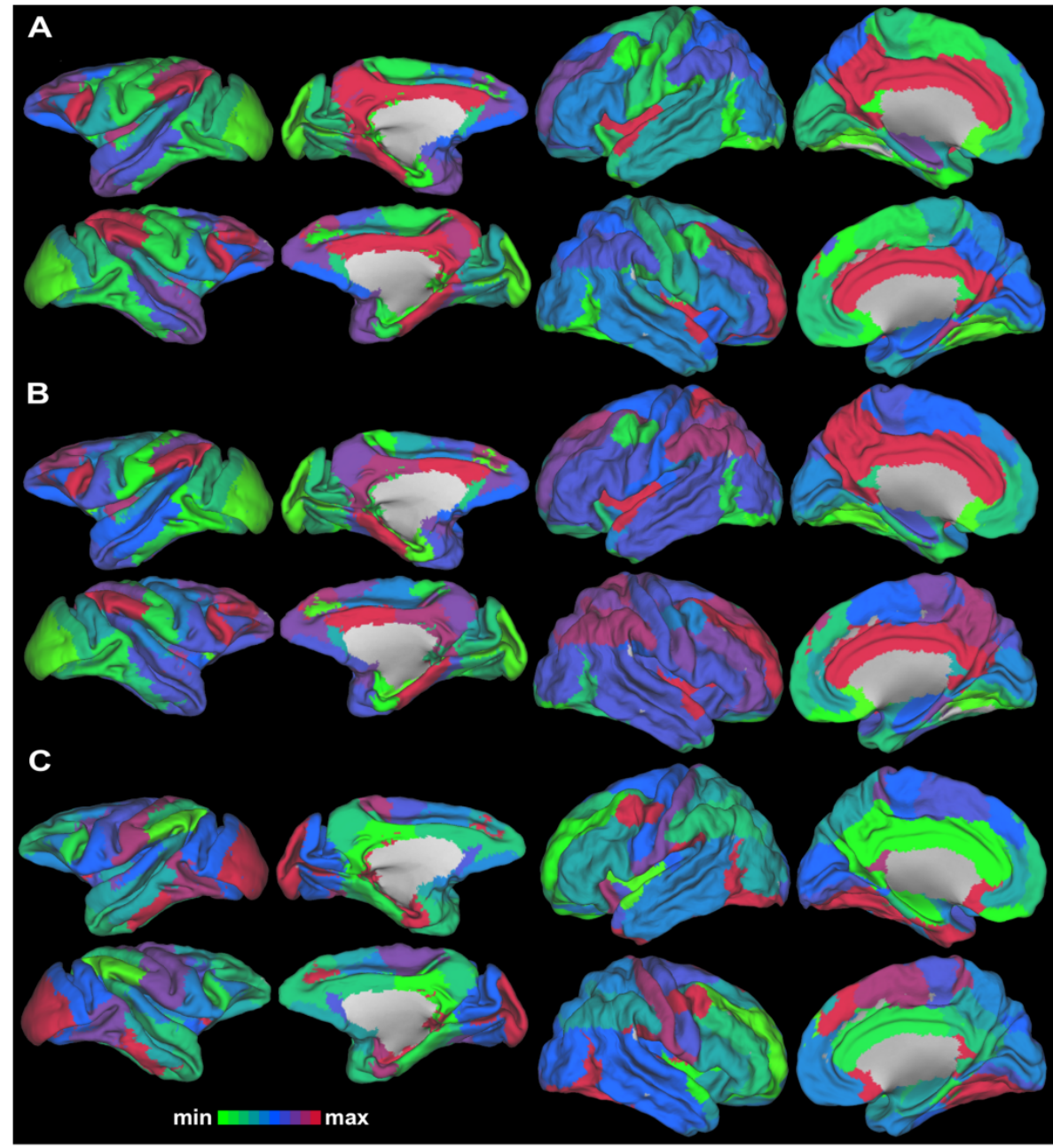

Figure 4: Centrality and clustering in the $\mathrm{MC}$ and $\mathrm{HC}$. A) BC B) EC and C) C values for the regions of the two species. Despite some common patterns, e.g. high centrality of cingulate cortex regions, these network metrics do not significantly correlate across the species (see Results).

The hemisphere-wise weighted analysis of EC, BC and C led to a comparable picture (Table S4) with no significant correlation between these metrics in the two species.

A significant RCC highlights the presence of a rich club organization in both the MC and HC (Fig. 5A, see also Fig. S3) in line 
with previous studies (Harriger et al., 2012; van den Heuvel and Sporns, 2011). Importantly, our direct comparative analysis that employed a parcellation scheme applicable to both species demonstrates that the regions forming a rich club exhibit a high and significant overlap (14/20), involving regions located at the frontal, parietal, cingulate and insular cortex (Fig. 5B and C, Table S5). This overlap is observed for a wide range within the rich club regime (Fig. 5C). This indicates that not only the macaque and human brain exhibits a rich club organization, but that this structure constitutes an evolutionarily preserved structural backbone involving a highly overlapping set of regions in both species.

Since the regions constituting a rich club have a high degree, i.e. number of connections, and the degree is positively related to $\mathrm{BC}$ and EC (Zuo et al., 2012), it is expected that the rich club regions will have higher $\mathrm{BC}$ and $\mathrm{EC}$ values when compared to non-rich club regions. We directly tested this prediction and found that rich club regions in both the MC (defining rich club and non-rich club regions by talking into account level $\mathrm{k}=56$ as a cutoff) and HC (defining rich club and non-rich club regions by talking into account level $\mathrm{k}=55$ as a cutoff) exhibit significantly higher $\mathrm{BC}$ and $\mathrm{EC}$ values when compared to non-rich club regions ( $p<0.001$, permutation tests). Moreover, comparing the $C$ values of rich club and non-rich club regions revealed the reversed relation: the rich club regions exhibited significantly lower $C$ values when compared to non-rich club regions ( $\mathrm{p}<0.001$, permutation tests). Hence, the regions of the "structural backbone" in both the MC and HC when compared with the rest of brain regions, appears highly central, further underlying their topological importance in mediating information across the brain. Moreover, they appear less segregated, indicating limited connections, and hence possible anatomical paths for "cross-talk", between the regions that they connect to.

Application of the weighted RCC to the left and right MC and HC separately, led to similar results (Table S6-S7). One notable exception was the failure of the weighted rich club analysis to reveal a 
statistically significant rich club in the left HC. Hemispheric differences in network metrics have been reported (Iturria-Medina et al., 2011) and this finding could signify a less prominent rich club structure in the left HC. However, given the high density of the network, and consequently a rather low cutoff used for considering connections in the $\mathrm{HC}$ to be taken into account, we suggest that this finding is the consequence of an inflated false positive rate obscuring the topology of the left HC.

For the whole brain analysis involving binary networks, control analysis gave rise to the following picture: The $\mathrm{L} x / \mathrm{L}$ ratio on perturbed networks revealed that despite a slight decrease, as expected since scrambling of the networks was introduced, from the $\mathrm{L}_{x} / \mathrm{L}$ ratio calculated between the original $\mathrm{MC}$ and $\mathrm{HC}$, the $\mathrm{Lx} / \mathrm{L}$ ratio remained significantly higher when compared to values obtained from random networks $\left(\mathrm{L}_{x} / \mathrm{L}\right.$ perturbed mean $=0.658 \mathrm{std}=0.003, \mathrm{~L} \times /$ Lnull mean $=0.599 \mathrm{std}=$ $0.006, \mathrm{p}<0.001$ null values from 1000 random networks). The HCS, HMIS, and RCC analysis from networks derived from the perturbation analysis revealed that the results are robust (Fig. S2, S3). This also held true for the control analysis of random insertion of connections. The usage of a different parcellation scheme (Bonin and Bailey, 1947) led to significant and converging results as the ones obtained for the RM $\left(\mathrm{L}_{x} /\right.$ Loriginal $=0.712 \mathrm{p}<0.001, \mathrm{~L} \times /$ Lnull mean $=0.558 \mathrm{std}=0.011$ null values from 1000 random networks, see also Table S8-S9). The choice of a different parcellation scheme gave rise to significant and comparable results, albeit with less regions reaching significance (Table S8), something that might be attributable to the fact that this map was not "designed" to be applicable in both species. Hence, the above results conjointly underscore the robustness and relative independence of the results from parcellation scheme choices. 

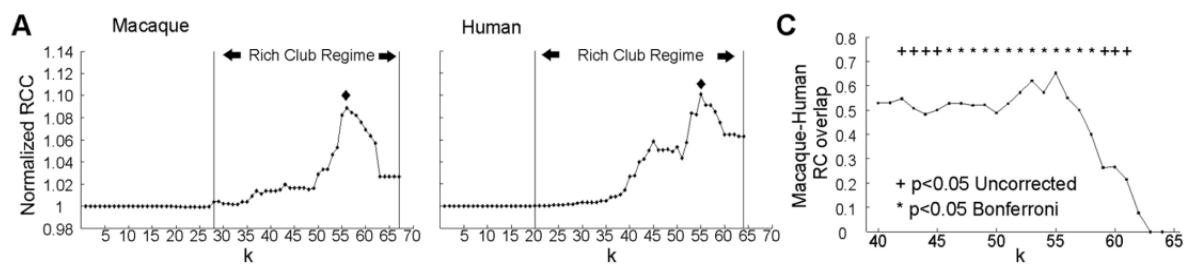

B

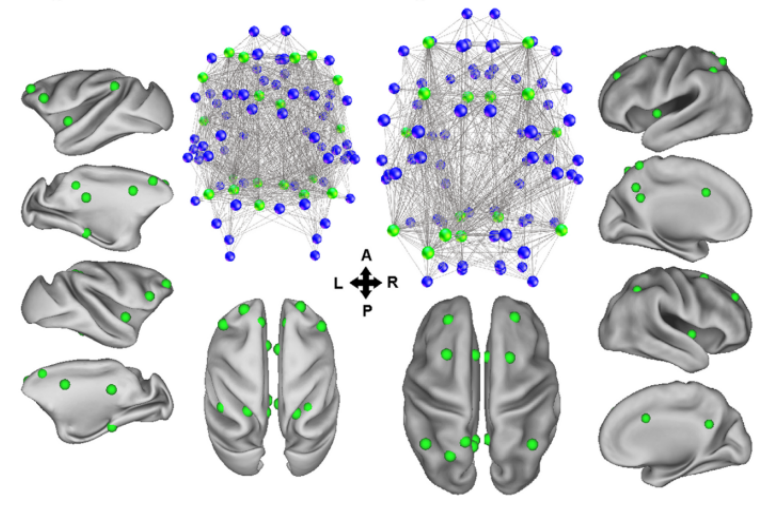

Figure 5: Rich club structure in the macaque and human brain. A) The normalized RCC suggests the presence of a rich club organization in both the human and macaque brains. For the unormalized curves see Fig. S3. The RCC obtained within the rich club regime (> 1) were significantly higher than the ones obtained from random networks matched for node, edge and degree distribution ( $\mathrm{p}<0.0001)$. B) Network and anatomical representation of the regions corresponding to the peak of the normalized RCC which is marked with a diamond in panel $\mathrm{A}$ ( $\mathrm{k}=56$ for macaque and $\mathrm{k}=55$ for human). In the network representation, green and blue nodes represent rich club and non-rich club regions at level $\mathrm{k}$ respectively. Only connections involving at least one rich club region are depicted. The anatomical representation depicts the regions constituting the rich club on the inflated fiducials of both hemispheres of the brains of the two species. The spheres represent the centre of mass of the regions. Note the convergence of the rich club analysis to a highly overlapping set of regions in the two species. C) The observed overlap is significant within a range of the rich club regime. The significance was established by drawing randomly from a uniform distribution a number of regions from the $\mathrm{MC}$ and $\mathrm{HC}$ equal to the number of regions corresponding at each level $\mathrm{k}$ for the $\mathrm{MC}$ and HC separately. The procedure was repeated 10000 times and the overlap of these randomly drawn regions was computed forming a null distribution with which the original overlap values were compared. 


\section{Discussion}

Prefrontal, parietal and cingulate regions

Cortical expansion of the human cortex in relation to the macaque is more prominent in prefrontal, parietal and cingulate regions (Hill et al., 2010). Our results suggest different degrees of perseverance of the macroscale connectivity of these regions during primate evolution.

An early view on the prefrontal cortex (PFC) suggests that it has been expanded in the lineage leading to humans (Brodmann, 1912; Campbell, 1905). Expansion of the human PFC relative to the macaque PFC is supported by contemporary investigations (Van Essen and Dierker, 2007) and is linked to unique human cognitive processes (Barbey et al., 2012). Moreover, PFC connectivity changes have also been proposed to underlie unique cognitive processes in humans (Semendeferi et al., 2002). A recent review (Petrides et al., 2012) as well as functional (Goulas et al., 2012) and structural (Thiebaut de Schotten et al., 2012) connectivity studies suggest comparable connections of the PFC of the two species. Additionally, quantitative analysis has revealed similar connectivity of macaque and human PFC regions with a small set of cortical regions (Croxson et al., 2005). However, pronounced changes are reported for the arcuate and inferior fronto-occipital fasciculi of humans and macaques (Rilling et al., 2008; Thiebaut de Schotten et al., 2012). Our study suggests a statistically significant preservation of distinct aspects of the wiring of several PFC regions across the species (Fig. 3, Table S2). Hence, unique features of the humans, i.e. "higher order cognitive processes/intelligence" attributed to the PFC, might not entail extensive reconfigurations of PFC connectivity in humans when compared to macaques.

The parietal cortex in macaques and humans consists of distinct subregions that are discernible on functional, connectional, macro- and microstructural criteria (Bonin and Bailey, 1947; Kotter and Wanke, 2005; Orban et al., 2006). Comparative studies reveal similarities but also 
some differences of the functional and connectional architecture of the parietal cortex subregions (Caspers et al., 2011; Mars et al., 2011; Orban et al., 2006). Our whole brain quantitative analyses offer complementary evidence by revealing that certain lateral parietal regions reach a statistically significant connectivity pattern similarity, while the medial parietal ones do not (Fig. 3, Table 1-S2). This could entail a functional similarity of lateral parietal regions between the two species and a divergence with respect to the medial ones.

The anterior cingulate cortex (CCa) exhibits extensive connections with parietal, motor, frontal, insular and limbic regions. Such connectivity renders it suitable for bridging the motivational, cognitive and motor domains (Morecraft et al., 2012). Functional evidence in humans and macaques pinpoint such an integrative role and involvement in decision making (Kaping et al., 2011; Rushworth et al., 2012). CCa is highly central and part of the rich club (Fig. 4-5, Table S3S4-S5-S7) a topological structural feature that might allow the involvement of this region in the aforementioned functional roles. Despite that CCa is part of the rich club in both species our results suggest a lack of preservation of its connectivity patterns (Fig. 3, Table 1, Table S2). This in turn might entail, alongside with potential preservance of certain functional properties, divergent functional roles of this region in the two species.

The posterior cingulate cortex (CCp) is a major node of the default mode network in both species, also involved in processes such as social cognition (Andrews-Hanna et al., 2010; Mantini et al., 2011; Mars et al., 2012). In addition, recent evidence from a functional study in humans suggests that this region exhibits dynamic properties subserving the integration of information from regions of distinct large scale networks (de Pasquale et al., 2012). The fact that CCp is central and part of the rich club in both species (Fig. 4-5, Table S3-S4-S5-S7) might constitute the structural basis for such integrative property reflected in functional measurements. Consequently, we hypothesize that such 
property will also hold for the macaque. However, the CCp appears to have not retained its connectivity with the rest of the brain (Fig. 3, Table 1-S2). Multimodal imaging of the macaque and human brain might be used to directly address if the aforementioned integrative functional property involving the CCp are common in the two species or a unique property of the human brain. Moreover, a possible rewiring of the CCp might have resulted in the reconfiguration of the neural circuitry, which seems also present in the macaque brain (Mars et al., 2012), underlying aspects of social cognition in humans.

\section{Centrality and clustering}

A general pattern was discernable in both species: high BC and EC values were observed in "association" cortices and low ones in "primary" cortices. The $\mathrm{C}$ values exhibited the reverse pattern (Fig. 4, Table S4). However, none of these network metrics appeared to persevere primate evolution, suggesting different levels of centrality and clustering at the whole brain level (see also Fig. S1). Nevertheless, regions in the cingulate cortices appeared highly central in both species (Fig. 4 A and B, Table S4) in line with previous findings (Bastiani et al., 2012; Harriger et al., 2012). Hence, cingulate cortex regions, despite the evidence for a different "wiring" in the two species (Fig. 3, Table S2), seem to have maintained their topological centrality, relevant for information integration.

Rich clubs: A common structural backbone in the macaque and human brain

Our analysis demonstrates the presence of a rich club organization in both the MC and HC (Fig. 5, Table S5-S6-S7-S9) confirming and extending previous findings (Harriger et al., 2012; van den Heuvel and Sporns, 2011). Importantly, our comparative approach allowed us to demonstrate that the regions forming a rich club are highly converging with a significant overlap within the rich club regime (Fig. $5 \mathrm{~B}$ and C, Table S5-S7-S9). Thus, this structural backbone is not only present in both macaques and humans, but also persevered through primate 
evolution, involving a highly overlapping set of regions in the two species. It is noteworthy, that the hemisphere-wise analysis failed to unveil a statistically robust rich club structure for the left HC. We believe that this is due to an increased false positive rate. However, a "laterality" might be present in the HC with respect to rich club organization, a potentiality demanding further future elaboration. Network analysis in the macaque (Harriger et al., 2012) and the human brain (van den Heuvel et al., 2012) revealed that the rich club connections are the most "costly", i.e. span long distances, and mediate traffic between distant regions through a sequence of short-long-short range structural pathways. Studies in the human brain indicate that inter-regional functional interactions are modulated by connection distance and take place within specific frequency bandwidths (Salvador et al., 2005; von Stein and Sarnthein, 2000). Additionally, macaque studies suggest a frequency-specific dialogue between two cortical regions that depends on the laminar origin and termination of the interregional connections (Bosman et al., 2012). Our comparative analysis can guide invasive techniques for the functional examination of the rich club regions of the MC. Such investigation is crucial for assessing if and how the aforementioned factors co-shape the functional dialogue within richclub and between rich club and non-rich club regions and thus highlight the principles that shape the flow of information through this structural backbone. Additionally, such functional investigation might unlock the mechanisms underlying the proposed role of rich-club regions in multisensory integration (Zamora-Lopez et al., 2010). Our comparative approach helps translating such functional findings to the human brain and develop hypothesis that could be tested with e.g. electrocorticography. In that way, future studies could assess if "homologous rich club" regions exhibit comparable and/or unique functional properties in the two species.

Lesions involving rich-club regions deteriorate the efficiency of the whole brain network and consequently can affect multiple cognitive 
domains as well as functional aspects like synchronization of functional networks (van den Heuvel and Sporns, 2011). The presence of a rich club structure involving highly overlapping regions in both MC and $\mathrm{HC}$ suggest that the macaque brain might be used as a model for e.g. studying the effects of lesions involving "homologous rich club" regions. However, certain common rich club regions, for instance CCp, lack significant interspecies connectivity similarity. Lesions in a brain region, apart from leading to the expected effects in regions directly connected to it, also lead to global effects through indirect connections (Young et al., 2000). Thus, if the wiring of the same lesioned regions differs, the lesion can lead to different global effects and consequently possibly different behavioural effects. The above conjointly, suggest that while lesioning common rich club regions will have detrimental global effects in both species, the nature and severity of such effects might depend on the degree of preservation of the connectivity of the involved regions.

\section{Factors responsible for connectivity discrepancies between the species}

Both genetic and environmental factors underlie system-level changes, including connectivity, of the cortex of mammals (Krubitzer and Kaas, 2005). For instance, functional connectivity differences observed between the inferior parietal lobule and anterior prefrontal cortex of macaques and humans can be the result of different foraging styles of the two species, dictated by different ecological factors which entail different challenges in decision making (Rushworth et al., 2012). Our results revealed statistically significant connectivity similarities between humans and macaques while absence thereof might suggest a rewiring also caused by the aforementioned factors. In addition, inaccuracies of the methods used and data incompleteness might also give rise to connectivity discrepancies (see Limitations and future directions). Thus, discrepancies might be attributed to "true" differences, methodological limitations and a mixture thereof. 
Does a statistically significant connectivity similarity necessarily entail functional similarity?

Both empirical and computational studies suggest that the connectivity of a region largely constrains its function (Passingham et al., 2002; Saygin et al., 2012). We have demonstrated the perseverance of the connectional patterns of certain assumed homologues in the two species in a quantitative way. Obviously humans and monkeys differ in certain cognitive functions e.g. language production. Hence, one intriguing question is the extent to which such a statistically significant perseverance of connectivity similarity is translated to similarity of function persevering evolution. Other factors apart from macroscale connectivity can shape the functional role of a region, e.g. laminar patterns of connections (Markov and Kennedy, 2013). Consequently, it could be the case that a statistically significant macroscale connectivity similarity of a region is not sufficient to guarantee evolutionary preserved functional similarity. In an analogous way, it has been demonstrated that the presence of an evolutionary conserved network can be accompanied by functional divergence (Mantini et al., 2013). Hence, while a statistical perseverance of macroscale connectivity suggests functional similarity, such a prediction demands explicit quantification in future studies. The network based methods employed in the current study in conjunction with data-driven methods for detecting cross-species functional homologies (Mantini et al., 2012) could be adopted in future studies for addressing the degree of convergence and divergence of connectional and functional similarity across the brain regions of the two species.

\section{Limitations and future directions}

Certain limitations should be taken into account when interpreting the findings of our study. First, while the expansion model is used extensively for interspecies comparisons, evidence suggests the presence of interspecies functional correspondences not predicted by it (Astafiev et al., 2003; Mantini et al., 2012). To perform interspecies comparisons 
without using the expansion model, dMRI and/or resting-state fMRI data collected in both species in conjunction with sophisticated techniques like network alignment (Berg and Lassig, 2006) can be used for an interspecies connectivity based region-to-region match. Moreover, connectivity based parcellation strategies can be adopted for parcellating the cortical mantle in a data-driven fashion (Goulas et al., 2012; Mars et al., 2011) without the need for an a priori defined parcellation scheme. This would allow addressing inter-species differences and similarities of connectional architecture at a more fine grained level going beyond the level of granularity currently adopted. However, performing connectivity based whole brain parcellation applicable to a comparative study remains challenging. Second, the MC was assembled through a meta-analysis of tracing studies, while the HC with the aid of dMRI. Good correspondence exists between the structural connections as revealed by tracers and diffusion imaging (Hagmann et al., 2008; Jbabdi et al., 2013; Schmahmann et al., 2007), but some inconsistencies are also discernible ( $\mathrm{Li}$ et al., 2012). Hence, we predict that the usage of $\mathrm{dMRI}$ for assembling the MC will lead to largely comparable results. Moreover, different weighting schemes for assembling the HC could also be adopted in the future (van den Heuvel and Sporns, 2011). Third, tractography methods have several limitations, like the limited detailed controlateral connectivity and the relation of false-positives and false negatives and connection distance, with longer connection distances appearing more prone to false negatives (Li et al., 2012). Hence, connections between distant regions might be underrepresented and might lead to lack of interspecies connectivity similarity (for a discussion see: Bastiani et al., 2012; Parker et al., 2013). Future studies employing the same modality for the estimation of connectivity in the two species, e.g. resting-state fMRI, will complement the current results. Forth, we currently used binary instead of weighted connectomes for the main analysis since certain network metrics currently employed (e.g. HCS) involve cross-matrix operations and the weights obtained from the different methods for assembling the 
MC and HC are not comparable. This restricted us from using all the metrics for the hemisphere-wise weighted analysis. Fifth, we compared the macroscale connectivity of the two species (Sporns et al., 2005). Apart from similarities and changes occurring at this level, connectivity changes between the species can occur at a mesoscale, i.e. connectivity at the laminar level (Preuss, 2000; Raghanti et al., 2008). Hence, a more complete understanding of interspecies differences requires quantitative comparative studies at multiple levels. Lastly, future incorporation of whole brain macroscale connectivity data from more primate species, e.g. apes, along with enrichment of existing connectivity databases and improvement of neuroinformatics tools (Bakker et al., 2012; Markov et al., 2011), will allow tracing the evolutionary trajectory of the primate brain in more detail. To this end, network metrics recently introduced for uncovering the structural backbone of the brain, such as coreperiphery analysis (Rombach et al., 2014), could also be used as an alternative to the metric, i.e. rich-club, currently employed.

\section{Conclusions}

We examined at the whole brain level the macroscale inter-regional structural connections of macaques and humans. While many similarities of the macroscale connectivity of the two species were observed, certain discrepancies were also present. This approach, which can be termed "comparative connectomics", offers closer interspecies comparisons and brings forth novel insights into the evolution of the connectional architecture of the primate brain. Thus, it constitutes a translational bridge, valuable for clinical, cognitive and systems neuroscience, between macaque and human research. 


\section{Acknowledgments}

We thank Peter De Weerd for a detailed feedback on a previous version of the manuscript. 


\section{References}

Andrews-Hanna, J.R., Reidler, J.S., Sepulcre, J., Poulin, R., Buckner, R.L., 2010. Functional-Anatomic Fractionation of the Brain's Default Network. Neuron 65, 550-562.

Astafiev, S.V., Shulman, G.L., Stanley, C.M., Snyder, A.Z., Van Essen, D.C., Corbetta, M., 2003. Functional organization of human intraparietal and frontal cortex for attending, looking, and pointing. Journal of Neuroscience 23, 4689-4699.

Bakker, R., Wachtler, T., Diesmann, M., 2012. CoCoMac 2.0 and the future of tracttracing databases. Front Neuroinform 6, 30.

Barbey, A.K., Colom, R., Solomon, J., Krueger, F., Forbes, C., Grafman, J., 2012. An integrative architecture for general intelligence and executive function revealed by lesion mapping. Brain 135, 1154-1164.

Bastiani, M., Shah, N.J., Goebel, R., Roebroeck, A., 2012. Human cortical connectome reconstruction from diffusion weighted MRI: the effect of tractography algorithm. Neuroimage 62, 1732-1749.

Berg, J., Lassig, M., 2006. Cross-species analysis of biological networks by Bayesian alignment. Proc Natl Acad Sci U S A 103, 10967-10972.

Bezgin, G., Vakorin, V.A., van Opstal, A.J., McIntosh, A.R., Bakker, R., 2012. Hundreds of brain maps in one atlas: registering coordinate-independent primate neuroanatomical data to a standard brain. Neuroimage 62, 67-76.

Bezgin, G., Wanke, E., Krumnack, A., Kotter, R., 2008. Deducing logical relationships between spatially registered cortical parcellations under conditions of uncertainty. Neural Networks 21, 1132-1145.

Bonacich, P., 1972. \{Factoring and weighting approaches to status scores and clique identification\}. Journal of Mathematical Sociology 2, 113-120.

Bonin, G.v., Bailey, P., 1947. The neocortex of Macaca mulatta. University of Illinois Press, Urbana,.

Bosman, C.A., Schoffelen, J.M., Brunet, N., Oostenveld, R., Bastos, A.M., Womelsdorf, T., Rubehn, B., Stieglitz, T., De Weerd, P., Fries, P., 2012. Attentional Stimulus Selection through Selective Synchronization between Monkey Visual Areas. Neuron 75, 875-888.

Bottger, J., Schafer, A., Lohmann, G., Villringer, A., Margulies, D.S., 2014. Threedimensional mean-shift edge bundling for the visualization of functional connectivity in the brain. IEEE Trans Vis Comput Graph 20, 471-480.

Bressler, S.L., Menon, V., 2010. Large-scale brain networks in cognition: emerging methods and principles. Trends Cogn Sci 14, 277-290.

Brodmann, K., 1909. Vergleichende Lokalisationslehre der Grosshirnrinde in ihren Prinzipien dargestellt auf Grund des Zellenbaues. 
Brodmann, K., 1912. Neue Ergebnisse über die vergleichende histologische Localisation der Grosshirnrinde mit besonderer Berücksichtigung des Stirnhirns. Anatomischer Anzeiger 41, 157-216.

Burns, G.A., Young, M.P., 2000. Analysis of the connectional organization of neural systems associated with the hippocampus in rats. Philos Trans R Soc Lond B Biol Sci 355, 55-70.

Campbell, A.W., 1905. Histological studies on the localisation of cerebral function. University Press, Cambridge,.

Campbell, C.B., Hodos, W., 1970. The concept of homology and the evolution of the nervous system. Brain Behav Evol 3, 353-367.

Caspers, S., Eickhoff, S.B., Rick, T., von Kapri, A., Kuhlen, T., Huang, R., Shah, N.J., Zilles, K., 2011. Probabilistic fibre tract analysis of cytoarchitectonically defined human inferior parietal lobule areas reveals similarities to macaques. Neuroimage 58, 362-380.

Colizza, V., Flammini, A., Serrano, M.A., Vespignani, A., 2006. Detecting rich-club ordering in complex networks. Nature Physics 2, 110-115.

Croxson, P.L., Johansen-Berg, H., Behrens, T.E., Robson, M.D., Pinsk, M.A., Gross, C.G., Richter, W., Richter, M.C., Kastner, S., Rushworth, M.F., 2005. Quantitative investigation of connections of the prefrontal cortex in the human and macaque using probabilistic diffusion tractography. J Neurosci 25, 8854-8866.

de Pasquale, F., Della Penna, S., Snyder, A.Z., Marzetti, L., Pizzella, V., Romani, G.L., Corbetta, M., 2012. A cortical core for dynamic integration of functional networks in the resting human brain. Neuron 74, 753-764.

Ercsey-Ravasz, M., Markov, N.T., Lamy, C., Van Essen, D.C., Knoblauch, K., Toroczkai, Z., Kennedy, H., 2013. A predictive network model of cerebral cortical connectivity based on a distance rule. Neuron 80, 184-197.

ffytche, D.H., Catani, M., 2005. Beyond localization: from hodology to function. Philos Trans R Soc Lond B Biol Sci 360, 767-779.

Fillard, P., Descoteaux, M., Goh, A., Gouttard, S., Jeurissen, B., Malcolm, J., RamirezManzanares, A., Reisert, M., Sakaie, K., Tensaouti, F., Yo, T., Mangin, J.F., Poupon, C., 2011. Quantitative evaluation of 10 tractography algorithms on a realistic diffusion MR phantom. Neuroimage 56, 220-234.

Freeman, L.C., 1978. Centrality in social networks conceptual clarification. Social Networks 1, 215-239.

Frey, S., Campbell, J.S., Pike, G.B., Petrides, M., 2008. Dissociating the human language pathways with high angular resolution diffusion fiber tractography. J Neurosci 28, 11435-11444.

Goulas, A., Uylings, H.B., Stiers, P., 2012. Unravelling the intrinsic functional organization of the human lateral frontal cortex: a parcellation scheme based on resting state fMRI. J Neurosci 32, 10238-10252. 
Hagmann, P., Cammoun, L., Gigandet, X., Meuli, R., Honey, C.J., Wedeen, V.J., Sporns, O., 2008. Mapping the structural core of human cerebral cortex. PLoS Biol 6, e159.

Harriger, L., van den Heuvel, M.P., Sporns, O., 2012. Rich club organization of macaque cerebral cortex and its role in network communication. PLoS One 7, e46497.

Hilgetag, C., Kötter, R., Stephan, K., Sporns, O., 2002. Computational Methods for the Analysis of Brain Connectivity. In: Ascoli, G. (Ed.), Computational Neuroanatomy. Humana Press, pp. 295-335.

Hill, J., Inder, T., Neil, J., Dierker, D., Harwell, J., Van Essen, D., 2010. Similar patterns of cortical expansion during human development and evolution. Proc Natl Acad Sci U S A 107, 13135-13140.

Iturria-Medina, Y., Fernandez, A.P., Morris, D.M., Canales-Rodriguez, E.J., Haroon, H.A., Penton, L.G., Augath, M., Garcia, L.G., Logothetis, N., Parker, G.J.M., MelieGarcia, L., 2011. Brain Hemispheric Structural Efficiency and Interconnectivity Rightward Asymmetry in Human and Nonhuman Primates. Cereb Cortex 21, 56-67.

Jbabdi, S., Lehman, J.F., Haber, S.N., Behrens, T.E., 2013. Human and monkey ventral prefrontal fibers use the same organizational principles to reach their targets: tracing versus tractography. J Neurosci 33, 3190-3201.

Jones, D.K., Knosche, T.R., Turner, R., 2013. White matter integrity, fiber count, and other fallacies: The do's and don'ts of diffusion MRI. Neuroimage 73, 239-254.

Kaping, D., Vinck, M., Hutchison, R.M., Everling, S., Womelsdorf, T., 2011. Specific Contributions of Ventromedial, Anterior Cingulate, and Lateral Prefrontal Cortex for Attentional Selection and Stimulus Valuation. PLoS Biol 9.

Kotter, R., Wanke, E., 2005. Mapping brains without coordinates. Philosophical Transactions of the Royal Society B-Biological Sciences 360, 751-766.

Krubitzer, L., Kaas, J., 2005. The evolution of the neocortex in mammals: how is phenotypic diversity generated? Curr Opin Neurobiol 15, 444-453.

Li, L.C., Rilling, J.K., Preuss, T.M., Glasser, M.F., Damen, F.W., Hu, X.P., 2012. Quantitative assessment of a framework for creating anatomical brain networks via global tractography. Neuroimage 61, 1017-1030.

Mantini, D., Corbetta, M., Romani, G.L., Orban, G.A., Vanduffel, W., 2013. Evolutionarily Novel Functional Networks in the Human Brain? Journal of Neuroscience 33, 3259-3275.

Mantini, D., Gerits, A., Nelissen, K., Durand, J.B., Joly, O., Simone, L., Sawamura, H., Wardak, C., Orban, G.A., Buckner, R.L., Vanduffel, W., 2011. Default Mode of Brain Function in Monkeys. Journal of Neuroscience 31, 12954-12962.

Mantini, D., Hasson, U., Betti, V., Perrucci, M.G., Romani, G.L., Corbetta, M., Orban, G.A., Vanduffel, W., 2012. Interspecies activity correlations reveal functional correspondence between monkey and human brain areas. Nature Methods 9, 277-U285. 
Markov, N.T., Kennedy, H., 2013. The importance of being hierarchical. Curr Opin Neurobiol 23, 187-194.

Markov, N.T., Misery, P., Falchier, A., Lamy, C., Vezoli, J., Quilodran, R., Gariel, M.A., Giroud, P., Ercsey-Ravasz, M., Pilaz, L.J., Huissoud, C., Barone, P., Dehay, C., Toroczkai, Z., Van Essen, D.C., Kennedy, H., Knoblauch, K., 2011. Weight consistency specifies regularities of macaque cortical networks. Cereb Cortex 21, 1254-1272.

Mars, R.B., Jbabdi, S., Sallet, J., O'Reilly, J.X., Croxson, P.L., Olivier, E., Noonan, M.P., Bergmann, C., Mitchell, A.S., Baxter, M.G., Behrens, T.E., Johansen-Berg, H., Tomassini, V., Miller, K.L., Rushworth, M.F., 2011. Diffusion-weighted imaging tractography-based parcellation of the human parietal cortex and comparison with human and macaque resting-state functional connectivity. J Neurosci 31, 4087-4100.

Mars, R.B., Neubert, F.X., Noonan, M.P., Sallet, J., Toni, I., Rushworth, M.F.S., 2012. On the relationship between the "default mode network" and the "social brain". Frontiers in Human Neuroscience 6.

Modha, D.S., Singh, R., 2010. Network architecture of the long-distance pathways in the macaque brain. Proc Natl Acad Sci U S A 107, 13485-13490.

Morecraft, R.J., Stilwell-Morecraft, K.S., Cipolloni, P.B., Ge, J., McNeal, D.W., Pandya, D.N., 2012. Cytoarchitecture and cortical connections of the anterior cingulate and adjacent somatomotor fields in the rhesus monkey. Brain Research Bulletin 87, 457-497.

Opsahl, T., Colizza, V., Panzarasa, P., Ramasco, J.J., 2008. Prominence and control: the weighted rich-club effect. Phys Rev Lett 101, 168702.

Orban, G.A., Claeys, K., Nelissen, K., Smans, R., Sunaert, S., Todd, J.T., Wardak, C., Durand, J.B., Vanduffel, W., 2006. Mapping the parietal cortex of human and nonhuman primates. Neuropsychologia 44, 2647-2667.

Orban, G.A., Van Essen, D., Vanduffel, W., 2004. Comparative mapping of higher visual areas in monkeys and humans. Trends Cogn Sci 8, 315-324.

Parker, G.D., Marshall, D., Rosin, P.L., Drage, N., Richmond, S., Jones, D.K., 2013. A pitfall in the reconstruction of fibre ODFs using spherical deconvolution of diffusion MRI data. Neuroimage 65, 433-448.

Parker, G.J.M., Haroon, H.A., Wheeler-Kingshott, C.A.M., 2003. A framework for a streamline-based probabilistic index of connectivity (PICo) using a structural interpretation of MRI diffusion measurements. Journal of Magnetic Resonance Imaging $18,242-254$.

Passingham, R., 2009. How good is the macaque monkey model of the human brain? Curr Opin Neurobiol 19, 6-11.

Passingham, R.E., Stephan, K.E., Kotter, R., 2002. The anatomical basis of functional localization in the cortex. Nat Rev Neurosci 3, 606-616.

Petrides, M., Tomaiuolo, F., Yeterian, E.H., Pandya, D.N., 2012. The prefrontal cortex: comparative architectonic organization in the human and the macaque monkey brains. Cortex 48, 46-57. 
Preuss, T.M., 2000. Taking the measure of diversity: comparative alternatives to the model-animal paradigm in cortical neuroscience. Brain Behav Evol 55, 287-299.

Raghanti, M.A., Stimpson, C.D., Marcinkiewicz, J.L., Erwin, J.M., Hof, P.R., Sherwood, C.C., 2008. Cortical dopaminergic innervation among humans, chimpanzees, and macaque monkeys: A comparative study. Neuroscience 155, 203-220.

Rao, A.R., Jana, R., Bandyopadhyay, S., 1996. A Markov Chain Monte Carlo Method for Generating Random $(0,1)$-Matrices with Given Marginals. Sankhyā: The Indian Journal of Statistics, Series A (1961-2002) 58, 225-242.

Rilling, J.K., Glasser, M.F., Preuss, T.M., Ma, X., Zhao, T., Hu, X., Behrens, T.E., 2008. The evolution of the arcuate fasciculus revealed with comparative DTI. Nat Neurosci 11, 426428.

Rombach, M.P., Porter, M.A., Fowler, J.H., Mucha, P.J., 2014. Core-Periphery Structure in Networks. Siam Journal on Applied Mathematics 74, 167-190.

Rubinov, M., Sporns, O., 2010. Complex network measures of brain connectivity: uses and interpretations. Neuroimage 52, 1059-1069.

Rushworth, M.F.S., Kolling, N., Sallet, J., Mars, R.B., 2012. Valuation and decisionmaking in frontal cortex: one or many serial or parallel systems? Curr Opin Neurobiol 22, 946-955.

Salvador, R., Suckling, J., Schwarzbauer, C., Bullmore, E., 2005. Undirected graphs of frequency-dependent functional connectivity in whole brain networks. Philos Trans $\mathrm{R}$ Soc Lond B Biol Sci 360, 937-946.

Saygin, Z.M., Osher, D.E., Koldewyn, K., Reynolds, G., Gabrieli, J.D., Saxe, R.R., 2012. Anatomical connectivity patterns predict face selectivity in the fusiform gyrus. Nat Neurosci 15, 321-327.

Schmahmann, J.D., Pandya, D.N., 2006. Fiber pathways of the brain. Oxford University Press, Oxford ; New York.

Schmahmann, J.D., Pandya, D.N., Wang, R., Dai, G., D'Arceuil, H.E., de Crespigny, A.J., Wedeen, V.J., 2007. Association fibre pathways of the brain: parallel observations from diffusion spectrum imaging and autoradiography. Brain 130, 630-653.

Semendeferi, K., Lu, A., Schenker, N., Damasio, H., 2002. Humans and great apes share a large frontal cortex. Nat Neurosci 5, 272-276.

Sereno, M.I., Tootell, R.B.H., 2005. From monkeys to humans: what do we now know about brain homologies? Curr Opin Neurobiol 15, 135-144.

Sporns, O., Honey, C.J., Kotter, R., 2007. Identification and classification of hubs in brain networks. PLoS One 2, e1049.

Sporns, O., Tononi, G., Kotter, R., 2005. The human connectome: A structural description of the human brain. PLoS Comput Biol 1, e42. 
Stephan, K.E., Zilles, K., Kotter, R., 2000. Coordinate-independent mapping of structural and functional data by objective relational transformation (ORT). Philosophical Transactions of the Royal Society of London Series B-Biological Sciences 355, 37-54.

Thiebaut de Schotten, M., Dell'Acqua, F., Valabregue, R., Catani, M., 2012. Monkey to human comparative anatomy of the frontal lobe association tracts. Cortex 48, 82-96.

Tournier, J.D., Calamante, F., Connelly, A., 2007. Robust determination of the fibre orientation distribution in diffusion MRI: Non-negativity constrained super-resolved spherical deconvolution. Neuroimage 35, 1459-1472.

van den Heuvel, M.P., Kahn, R.S., Goni, J., Sporns, O., 2012. High-cost, high-capacity backbone for global brain communication. Proc Natl Acad Sci U S A 109, 11372-11377.

van den Heuvel, M.P., Mandl, R.C., Stam, C.J., Kahn, R.S., Hulshoff Pol, H.E., 2010. Aberrant frontal and temporal complex network structure in schizophrenia: a graph theoretical analysis. J Neurosci 30, 15915-15926.

van den Heuvel, M.P., Sporns, O., 2011. Rich-club organization of the human connectome. J Neurosci 31, 15775-15786.

Van Essen, D.C., 2004. Surface-based approaches to spatial localization and registration in primate cerebral cortex. Neuroimage 23 Suppl 1, S97-107.

Van Essen, D.C., Dierker, D.L., 2007. Surface-based and probabilistic atlases of primate cerebral cortex. Neuron 56, 209-225.

Van Essen, D.C., Glasser, M.F., Dierker, D.L., Harwell, J., 2012a. Cortical parcellations of the macaque monkey analyzed on surface-based atlases. Cereb Cortex 22, 2227-2240.

Van Essen, D.C., Glasser, M.F., Dierker, D.L., Harwell, J., Coalson, T., 2012b. Parcellations and hemispheric asymmetries of human cerebral cortex analyzed on surface-based atlases. Cereb Cortex 22, 2241-2262.

von Stein, A., Sarnthein, J., 2000. Different frequencies for different scales of cortical integration: from local gamma to long range alpha/theta synchronization. Int J Psychophysiol 38, 301-313.

Watts, D.J., Strogatz, S.H., 1998. Collective dynamics of 'small-world' networks. Nature $393,440-442$.

Young, M.P., Hilgetag, C.C., Scannell, J.W., 2000. On imputing function to structure from the behavioural effects of brain lesions. Philos Trans R Soc Lond B Biol Sci 355, 147-161.

Zamora-Lopez, G., Zhou, C., Kurths, J., 2010. Cortical hubs form a module for multisensory integration on top of the hierarchy of cortical networks. Front Neuroinform 4,1 . 
Zuo, X.N., Ehmke, R., Mennes, M., Imperati, D., Castellanos, F.X., Sporns, O., Milham, M.P., 2012. Network centrality in the human functional connectome. Cereb Cortex 22, 1862-1875. 


\section{Supplementary Material}

A

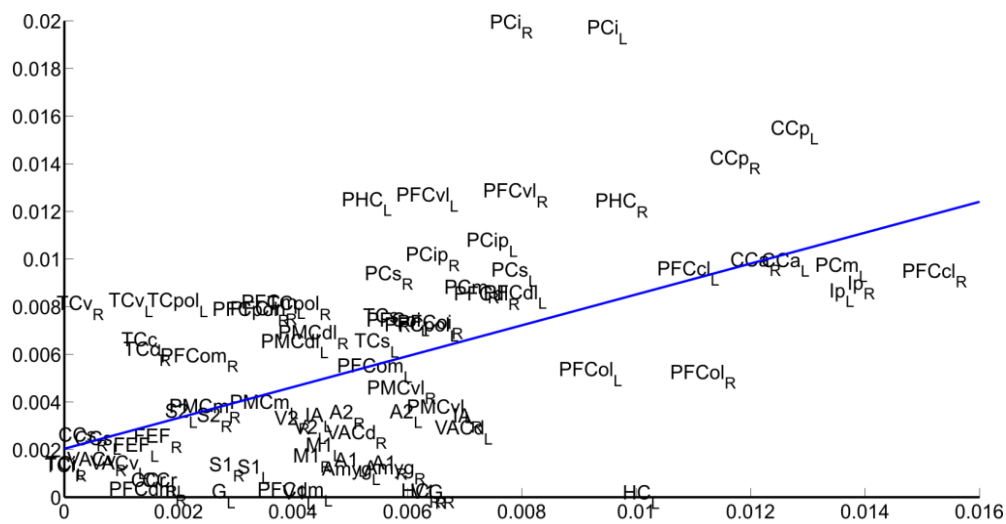

B

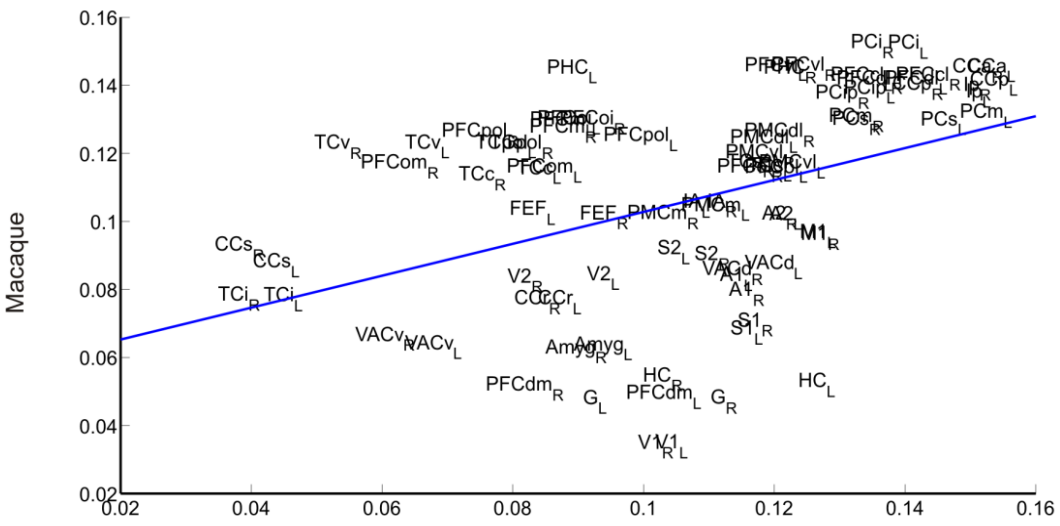

C

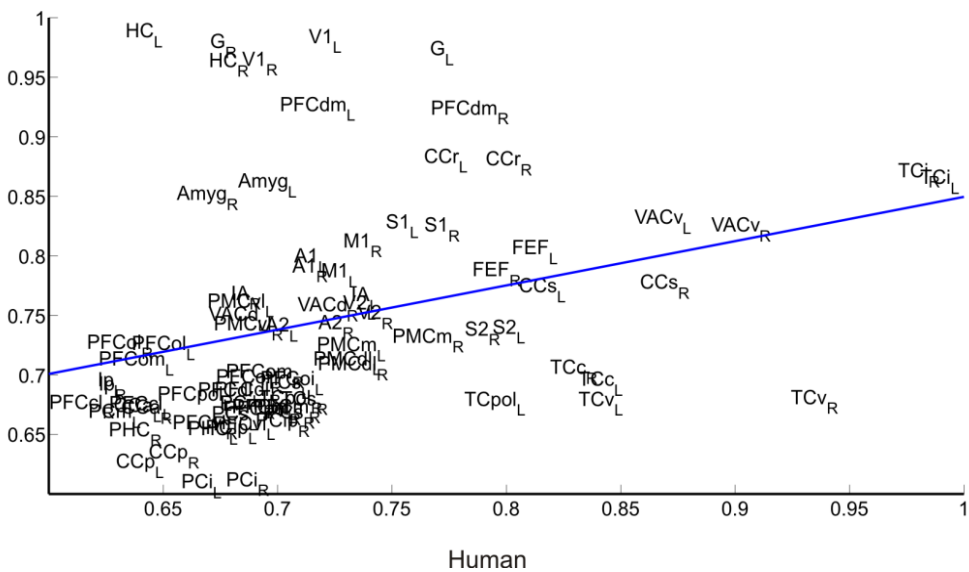

Figure S1: Scatter plots of centrality and clustering values for the $\mathrm{MC}$ and HC. A. BC B. EC and C. C values. Lines represent a least-square fit. Correlations between values obtained from the MC and HC were not significant (see Results). 
A

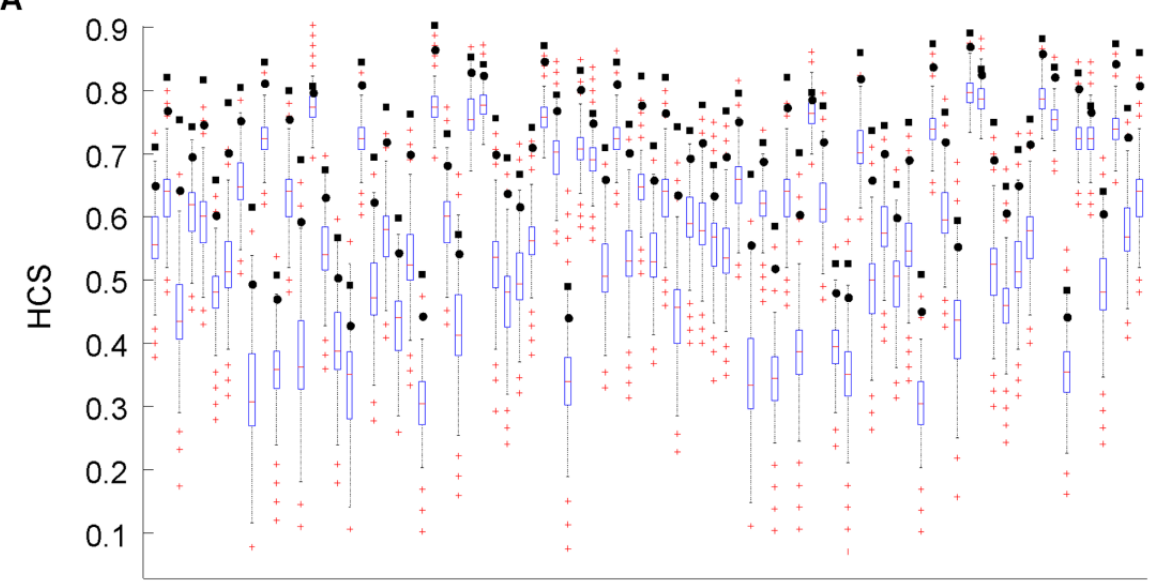

B

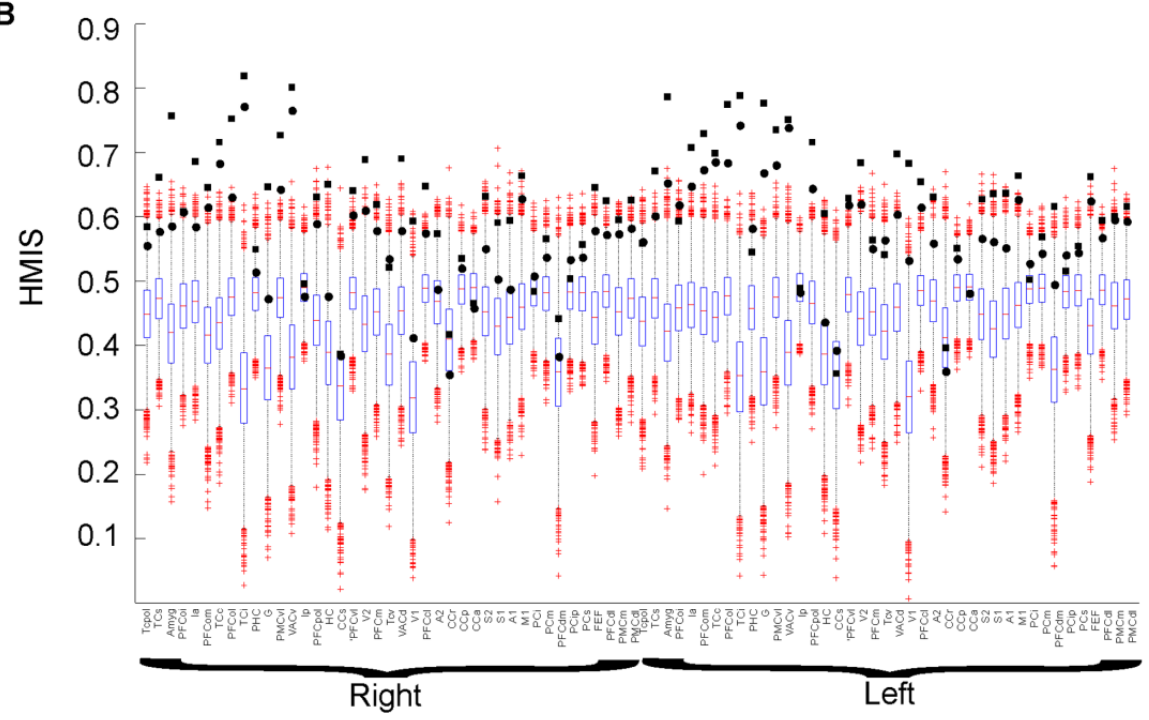

Figure S2: Region-wise values obtained for A. HCS and B. HMIS. Black squares and black circles represent the values obtained from the unscrambled and scrambled MC and $\mathrm{HC}$ respectively. Box plots represent the null values obtained from matched random networks and depict the median, $25 \%$ and $75 \%$ quantiles, and outliers of the null values. 


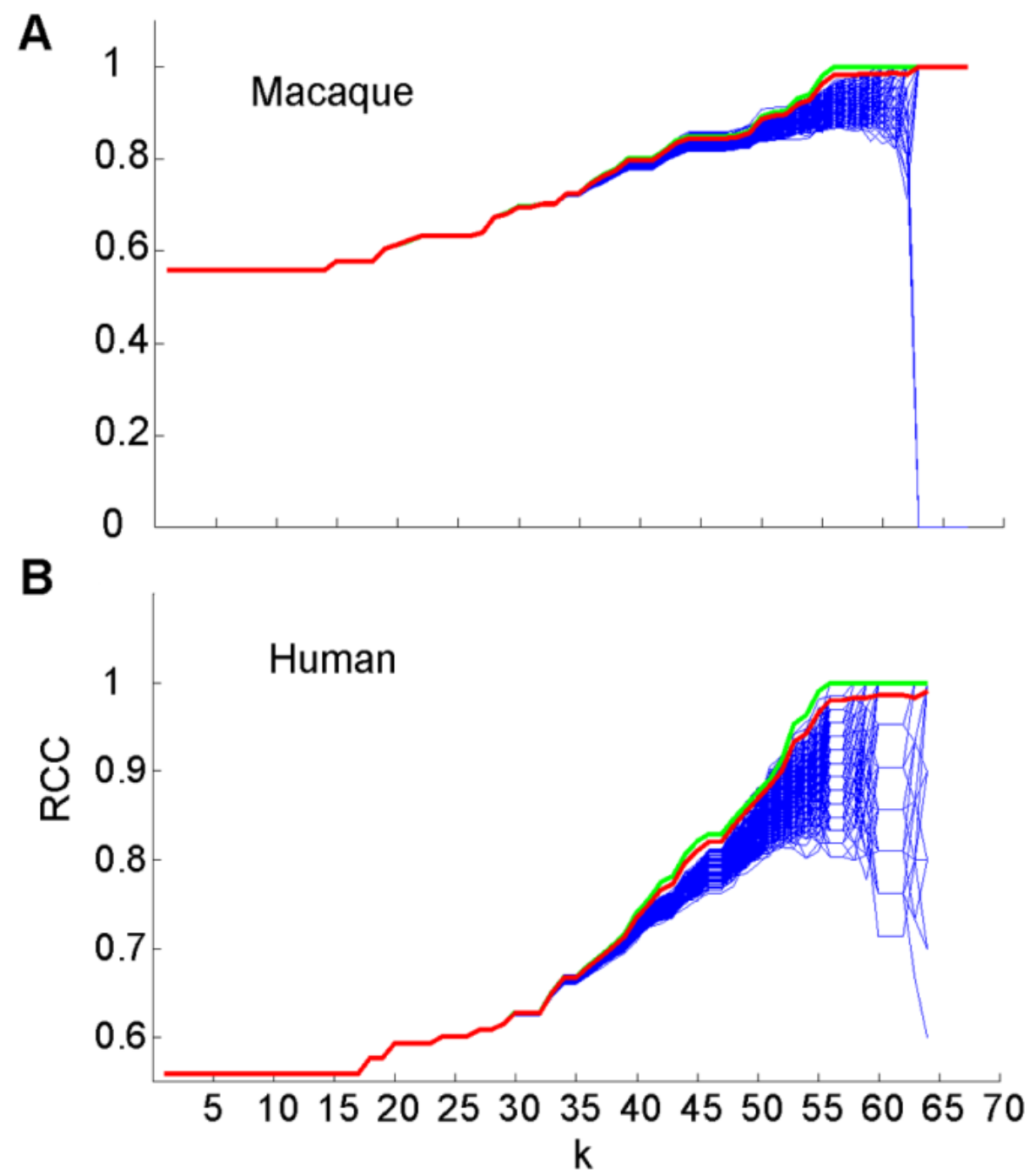

Figure S3: curves depicting the values for the RCC for a range of $k$ values for both A. $\mathrm{MC}$ and B. HC. Green curves and red curves correspond to values obtained from the unscrambled and scrambled networks respectively. Blue curves correspond to values obtained from matched random networks. Note that both the green and red curves lie above the blue ones that represent the null values for the RCC. 


\begin{tabular}{|c|c|c|c|}
\hline RM acronyms & Full name & Cortical Region & Cortical Type \\
\hline PFCoi & orbito intermediate prefrontal cortex & $\mathrm{F}$ & Iso \\
\hline PFCom & orbitomedial prefrontal cortex & $\mathrm{F}$ & Iso \\
\hline PFCol & orbitolateral prefrontal cortex & $\mathrm{F}$ & Iso \\
\hline G & gustatory cortex & $\mathrm{F}$ & Iso \\
\hline PMCvl & ventrolateral premotor cortex & $\mathrm{F}$ & Iso \\
\hline PFCpol & prefrontal polar cortex & $\mathrm{F}$ & Iso \\
\hline PFCvl & ventrolateral prefrontal cortex & $\mathrm{F}$ & Iso \\
\hline PFCm & medial prefrontal cortex & $\mathrm{F}$ & Iso \\
\hline PFCcl & centrolateral prefrontal cortex & $\mathrm{F}$ & Iso \\
\hline M1 & primary motor cortex & $\mathrm{F}$ & Iso \\
\hline PFCdm & dorsomedial prefrontal cortex & $\mathrm{F}$ & Iso \\
\hline FEF & frontal eye field & $\mathrm{F}$ & Iso \\
\hline PFCdl & dorsolateral prefrontal cortex & $\mathrm{F}$ & Iso \\
\hline $\mathrm{PMCm}$ & medial premotor cortex & $\mathrm{F}$ & Iso \\
\hline PMCdl & dorsolateral premotor cortex & $\mathrm{F}$ & Iso \\
\hline Tcpol & temporal polar cortex & $\mathrm{T}$ & Iso \\
\hline TCs & superior temporal cortex & $\mathrm{T}$ & Iso \\
\hline TCc & central temporal cortex & $\mathrm{T}$ & Iso \\
\hline $\mathrm{TCi}$ & inferior temporal cortex & $\mathrm{T}$ & Iso \\
\hline TCv & ventral temporal cortex & $\mathrm{T}$ & Iso \\
\hline A1 & primary auditory cortex & $\mathrm{T}$ & Iso \\
\hline A2 & secondary auditory cortex & $\mathrm{T}$ & Iso \\
\hline S1 & primary somatosensory cortex & $P$ & Iso \\
\hline $\mathrm{S} 2$ & secondary somatosensory cortex & $P$ & Iso \\
\hline $\mathrm{PCi}$ & inferior parietal cortex & $P$ & Iso \\
\hline $\mathrm{PCm}$ & medial parietal cortex & $P$ & Iso \\
\hline PCip & intraparietal cortex & $\mathrm{P}$ & Iso \\
\hline PCs & superior parietal cortex & $P$ & Iso \\
\hline V1 & visual area 1 (primary visual cortex) & 0 & Iso \\
\hline V2 & visual area 2 (secondary visual cortex) & 0 & Iso \\
\hline VACd & anterior visual area, dorsal part & $\mathrm{O}$ & Iso \\
\hline VACV & anterior visual area, ventral part & 0 & Iso \\
\hline Amyg & amygdala & L & Sub \\
\hline $\mathrm{PHC}$ & parahippocampal cortex & L & Allo \\
\hline $\mathrm{HC}$ & hippocampus & L & Allo \\
\hline CCs & subgenual cingulate cortex & L & Allo \\
\hline $\mathrm{CCr}$ & retrosplenial cingulate cortex & $\mathrm{L}$ & Allo \\
\hline CCp & posterior cingulate cortex & L & Allo \\
\hline $\mathrm{CCa}$ & anterior cingulate cortex & L & Allo \\
\hline Ia & anterior insula & I & Iso \\
\hline Ip & posterior insula & I & Iso \\
\hline
\end{tabular}

Table S1: RM acronyms and full names of the regions constituting the whole brain parcellation used in the current study. Each region is classified as allocortical (Allo), isocortical (Iso) or subcortical (Sub). An assignment is also provided based on a 5 way division $(\mathrm{F}=$ frontal, $\mathrm{T}=$ temporal, $\mathrm{P}=$ parietal, $\mathrm{O}=$ occipital, $\mathrm{L}=$ limbic, $\mathrm{I}=$ insular $)$. 


\begin{tabular}{lcccc}
\hline RM acronym & \multicolumn{2}{c}{ Right } & \multicolumn{2}{c}{ Left } \\
& z-score & p-value & z-score & p-value \\
\hline PFCoi & $\mathbf{2 . 8 1}$ & $\mathbf{0 . 0 0 0 9}$ & 2.01 & 0.0177 \\
PFCom & $\mathbf{2 . 6 7}$ & $\mathbf{0 . 0 0 1 7}$ & $\mathbf{2 . 6 0}$ & $\mathbf{0 . 0 0 1 6}$ \\
PFCol & 1.72 & 0.0338 & 0.95 & 0.1750 \\
G & 1.41 & 0.0741 & 1.27 & 0.0972 \\
PMCvl & $\mathbf{2 . 4 2}$ & $\mathbf{0 . 0 0 3 6}$ & $\mathbf{2 . 3 4}$ & $\mathbf{0 . 0 0 3 9}$ \\
PFCpol & 1.81 & 0.0270 & 1.56 & 0.0528 \\
PFCvl & 1.49 & 0.0599 & 0.95 & 0.1762 \\
PFCm & 1.71 & 0.0391 & 1.33 & 0.0851 \\
PFCcl & 1.05 & 0.1441 & 0.80 & 0.2179 \\
M1 & $\mathbf{3 . 4 8}$ & $\mathbf{0 . 0 0 0 1}$ & $\mathbf{4 . 0 3}$ & $\mathbf{0 . 0 0 0 1}$ \\
PFCdm & 0.53 & 0.3086 & 1.43 & 0.0725 \\
FEF & 1.38 & 0.0806 & 1.91 & 0.0195 \\
PFCdl & 0.84 & 0.2071 & 0.84 & 0.2083 \\
PMCm & $\mathbf{2 . 1 0}$ & $\mathbf{0 . 0 1 1 1}$ & $\mathbf{2 . 3 8}$ & $\mathbf{0 . 0 0 4 1}$ \\
PMCdl & $\mathbf{2 . 1 8}$ & $\mathbf{0 . 0 0 9 5}$ & $\mathbf{2 . 4 4}$ & $\mathbf{0 . 0 0 3 4}$ \\
Tcpol & $\mathbf{2 . 0 9}$ & $\mathbf{0 . 0 1 3 2}$ & 1.66 & 0.0439 \\
TCs & 0.50 & 0.3181 & 0.34 & 0.3769 \\
TCc & 1.48 & 0.0634 & 1.34 & 0.0893 \\
TCi & $\mathbf{1 . 9 7}$ & $\mathbf{0 . 0 1 6 3}$ & 1.71 & 0.0364 \\
TCv & 0.68 & 0.2555 & 0.60 & 0.2880 \\
A1 & 0.64 & 0.2676 & 0.51 & 0.3173 \\
A2 & 1.22 & 0.1097 & 1.02 & 0.1586 \\
S1 & $\mathbf{3 . 6 0}$ & $\mathbf{0 . 0 0 0 1}$ & $\mathbf{3 . 8 3}$ & $\mathbf{0 . 0 0 0 1}$ \\
S2 & 1.80 & 0.0296 & 1.75 & 0.0322 \\
PCi & 1.82 & 0.0280 & 1.66 & 0.0417 \\
PCm & 0.43 & 0.3457 & 0.73 & 0.2399 \\
PCip & $\mathbf{2 . 3 2}$ & $\mathbf{0 . 0 0 6 5}$ & $\mathbf{2 . 1 2}$ & $\mathbf{0 . 0 1 1 3}$ \\
PCs & $\mathbf{2 . 3 6}$ & $\mathbf{0 . 0 0 6 4}$ & $\mathbf{2 . 5 3}$ & $\mathbf{0 . 0 0 2 1}$ \\
V1 & $\mathbf{3 . 6 4}$ & $\mathbf{0 . 0 0 0 1}$ & $\mathbf{3 . 6 7}$ & $\mathbf{0 . 0 0 0 1}$ \\
V2 & $\mathbf{3 . 0 3}$ & $\mathbf{0 . 0 0 0 2}$ & $\mathbf{2 . 7 4}$ & $\mathbf{0 . 0 0 1 2}$ \\
VACd & $\mathbf{2 . 4 7}$ & $\mathbf{0 . 0 0 3 6}$ & $\mathbf{2 . 6 3}$ & $\mathbf{0 . 0 0 2 2}$ \\
VACv & $\mathbf{3 . 1 4}$ & $\mathbf{0 . 0 0 0 1}$ & $\mathbf{2 . 9 6}$ & $\mathbf{0 . 0 0 0 4}$ \\
Amyg & $\mathbf{2 . 8 2}$ & $\mathbf{0 . 0 0 0 6}$ & $\mathbf{2 . 5 3}$ & $\mathbf{0 . 0 0 2 4}$ \\
PHC & 0.33 & 0.3826 & -0.02 & 0.5228 \\
HC & $\mathbf{2 . 3 5}$ & $\mathbf{0 . 0 0 5 1}$ & $\mathbf{2 . 1 8}$ & $\mathbf{0 . 0 0 7 8}$ \\
CCs & $\mathbf{1 . 8 8}$ & $\mathbf{0 . 0 1 7 8}$ & 1.82 & 0.0236 \\
CCr & -0.33 & 0.6411 & -0.37 & 0.6574 \\
CCp & -0.48 & 0.6909 & -0.07 & 0.5421 \\
CCa & -0.03 & 0.5252 & -0.24 & 0.6111 \\
Ia & 1.97 & 0.0213 & 1.39 & 0.0837 \\
Ip & 0.91 & 0.1884 & 0.30 & 0.3887 \\
\hline P & & & & \\
\hline
\end{tabular}

Table S2: weighted HMIS results of the hemisphere wise analysis for the RM regions. Regions exhibiting significant HMIS values ( $\mathrm{p}<0.05$ FDR corrected) are in bold. The $\mathrm{p}-$ values and z-scores of the aforementioned metrics are also depicted. 


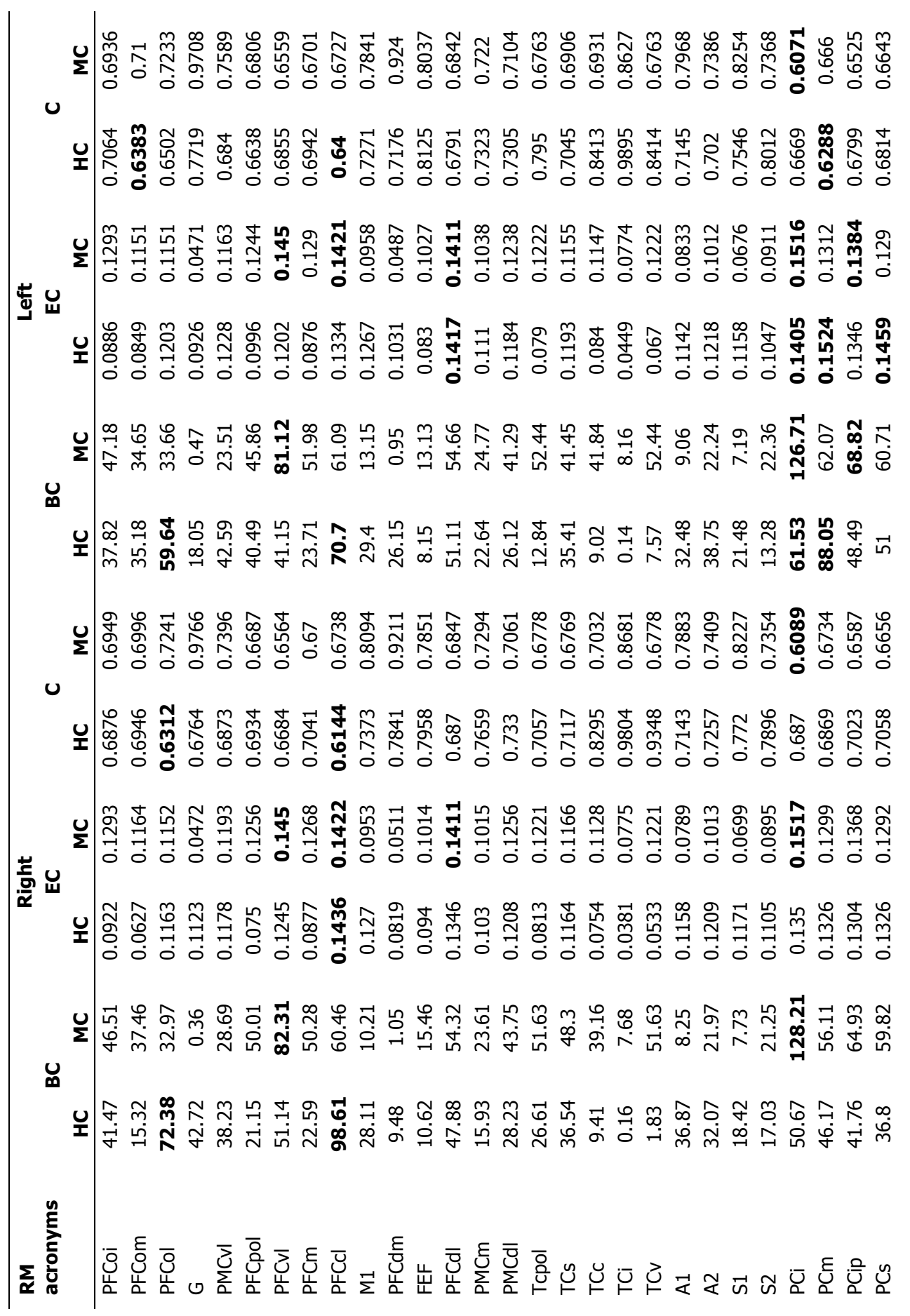




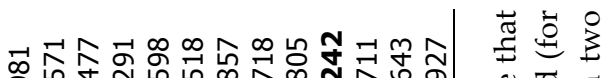

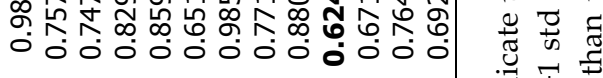

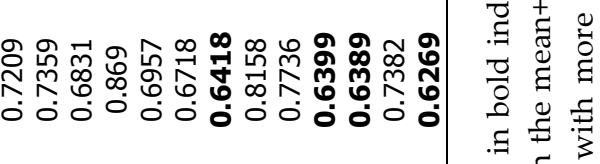

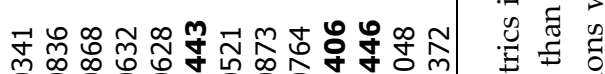

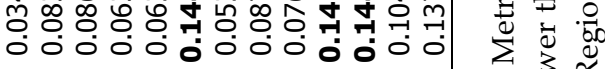

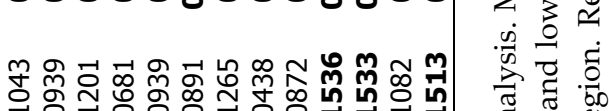

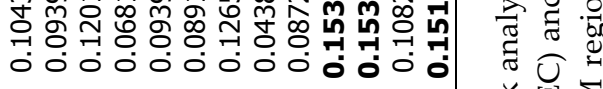

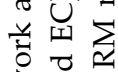

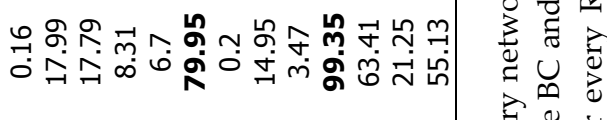

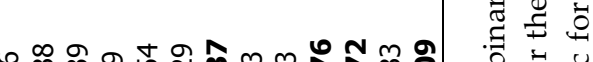
ஸे

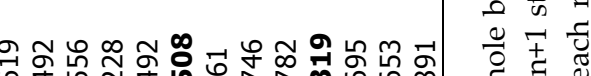

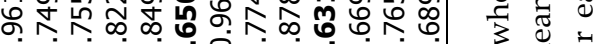

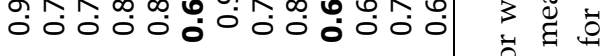

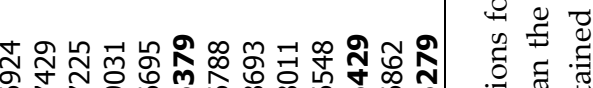

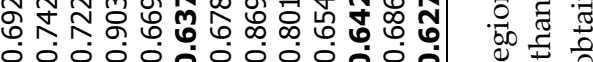

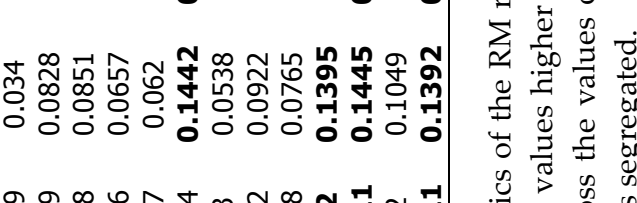

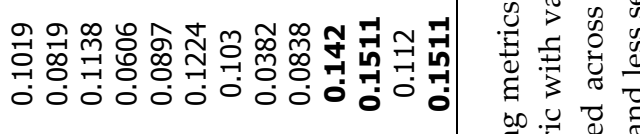
-

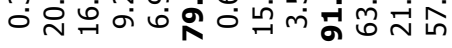

กิ 00.

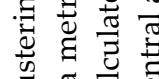
ป 둘. 굴

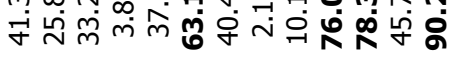
跤

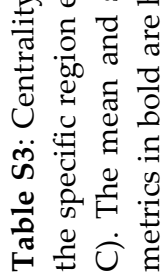




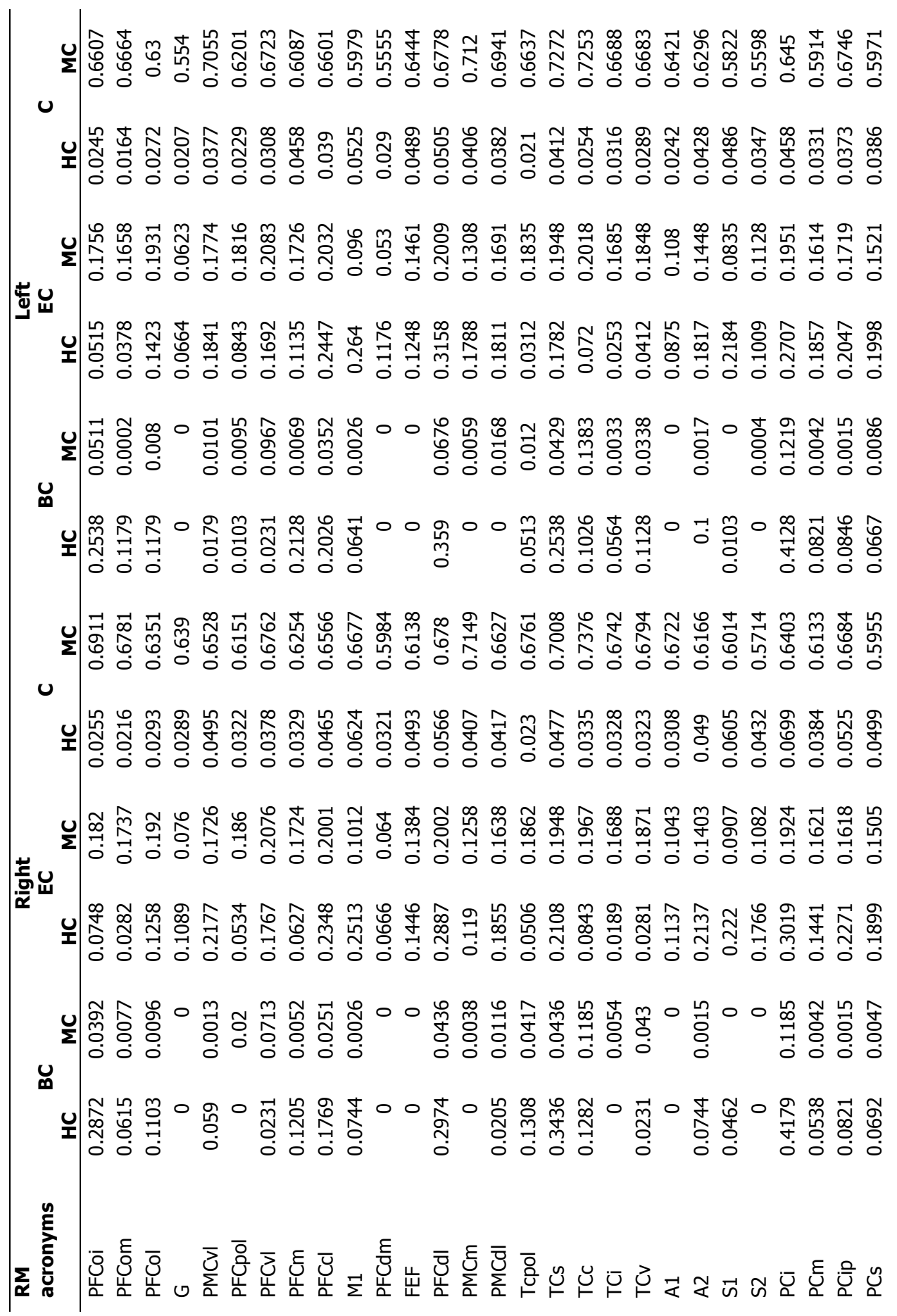


m

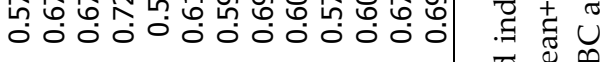

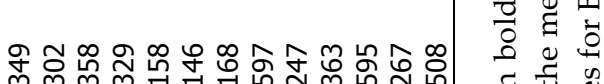

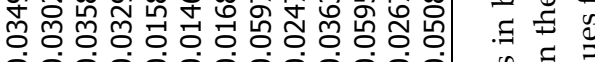

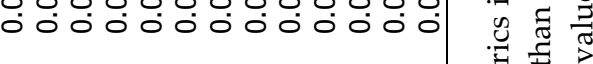

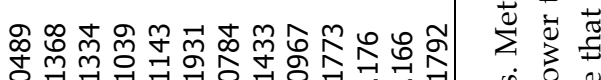

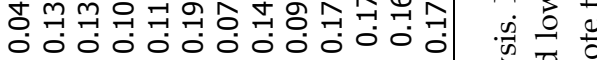

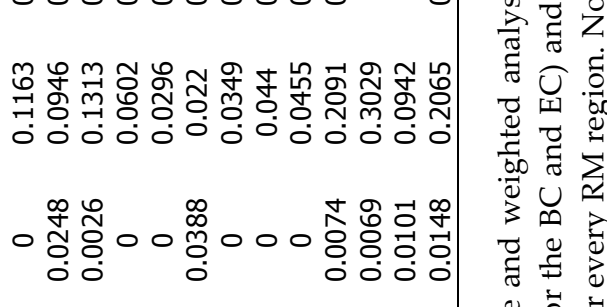

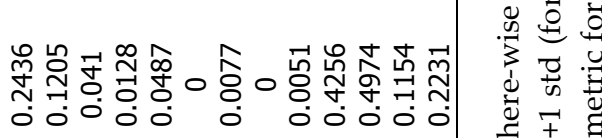

市

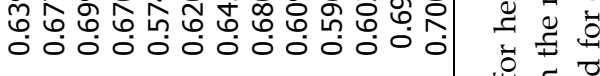

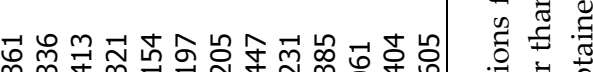

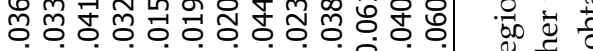
○ं0

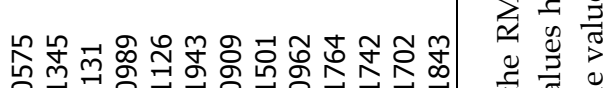


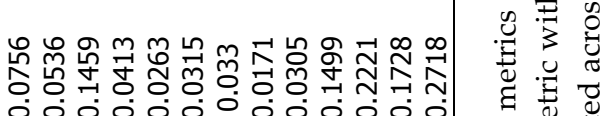
○旁芯 ○

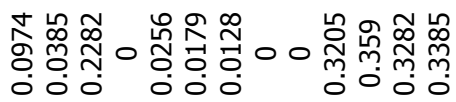

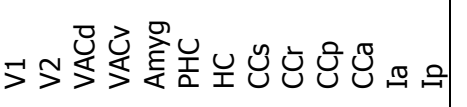

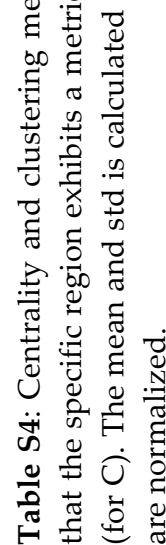




\begin{tabular}{|c|c|c|c|c|}
\hline & \multirow[b]{2}{*}{ RM acronyms } & \multicolumn{3}{|c|}{ Coordinates of centre of mass } \\
\hline & & $\mathbf{x}$ & $\mathbf{y}$ & $\mathbf{z}$ \\
\hline \multirow[t]{19}{*}{ Macaque } & PHC_R & 13.26 & -20.92 & -7.1 \\
\hline & Ip_R & 21.3 & -4.81 & -1.10 \\
\hline & PFCVl_R & 19.31 & 9.24 & 7.95 \\
\hline & PFCcl_R & 11.41 & 15.55 & 12.9 \\
\hline & CCp_R & 3.52 & -21.3 & 9.1 \\
\hline & CCa_R & 2.46 & 1.66 & 12.8 \\
\hline & PCi_R & 16.73 & -22.96 & 16.8 \\
\hline & PCip_R & 9.15 & -23.75 & 13.9 \\
\hline & PFCdI_R & 6.4 & 13.91 & 18.3 \\
\hline & PHC_ $\bar{L}$ & -13.02 & -20.78 & -7.91 \\
\hline & Ip_L & -21.42 & -2.39 & -1.19 \\
\hline & PFCVI_L & -20.1 & 9.86 & 8.9 \\
\hline & PFCcl_L & -12.1 & 16.47 & 12.1 \\
\hline & CCp_L & -4.23 & -20.98 & 8.44 \\
\hline & CCa_L & -3.54 & 4.11 & 13 \\
\hline & $\mathbf{P C i} \_\mathbf{L}$ & -17.3 & -23.33 & 16.1 \\
\hline & PCm_L & -3.4 & -26.52 & 14.9 \\
\hline & PCip_L & -10.65 & -22.37 & 13.8 \\
\hline & PFCdI_L & -7.58 & 14.2 & 17.4 \\
\hline \multirow[t]{15}{*}{ Human } & Ip_R & 42.83 & -4.1 & 1.42 \\
\hline & PFCcl_R & 25.43 & 40.83 & 34.79 \\
\hline & CCp_R & 9.92 & -48.92 & 26.33 \\
\hline & CCa_R & 6 & 13.65 & 30.61 \\
\hline & PCi_R & 41.62 & -53.39 & 42.95 \\
\hline & PFCdI_R & 24.8 & 12.89 & 53.77 \\
\hline & Ip_L & -42.92 & -4.18 & 1.28 \\
\hline & PFCCl_L & -25.44 & 40.87 & 34.8 \\
\hline & CCp_L & -9.86 & -48.9 & 26.3 \\
\hline & CCa_L & -5.68 & 13.53 & 30.18 \\
\hline & PCi_L & -41.67 & -53.46 & 43.03 \\
\hline & PCm_L & -8.34 & -56.88 & 39.65 \\
\hline & PCip_L & -22.64 & -62 & 56.03 \\
\hline & PCs_L & -13.93 & -52.85 & 60.79 \\
\hline & PFCdI_L & -24.7 & 13.16 & 53.91 \\
\hline
\end{tabular}

Table S5: List of regions constituting the rich club in macaque and human for whole brain binary network analysis. The set of regions corresponds to the level $\mathrm{k}$ for which the maximum normalized RCC was observed ( $\mathrm{k}=56$ for macaque and $\mathrm{k}=55$ for human) (see Fig. 4). Regions in bold denote rich club regions common in both species. Coordinates for the macaque correspond to the F99 space and for the human to MNI space. The suffixes $\mathrm{R}$ and $\mathrm{L}$ denote the right and left hemisphere. 


\begin{tabular}{|c|c|c|c|c|}
\hline \multirow[b]{2}{*}{ Macaque Left } & \multirow[b]{2}{*}{ RM acronyms } & \multicolumn{3}{|c|}{ Coordinates } \\
\hline & & $\mathbf{x}$ & y & $\mathbf{z}$ \\
\hline & Tcpol & -16.11 & 0.12 & -15.6904 \\
\hline & Tcs & -24.42 & -11.19 & -0.3752 \\
\hline & PFCoi & -11.04 & 6.16 & -1.5743 \\
\hline & Ia & -17.61 & 3.28 & -3.4493 \\
\hline & PFCom & -4.73 & 13.30 & 1.6570 \\
\hline & Tcc & -23.59 & -14.60 & -4.5224 \\
\hline & PFCol & -17.58 & 15.55 & 8.4587 \\
\hline & Tci & -24.52 & -13.53 & -13.0387 \\
\hline & PHC & -13.02 & -20.79 & -7.9199 \\
\hline & PMCvl & -22.70 & 4.27 & 6.2231 \\
\hline & Ip & -21.43 & -2.39 & -1.1984 \\
\hline & PFCpol & -5.25 & 24.64 & 9.7107 \\
\hline & PFCvl & -20.11 & 9.86 & 8.9091 \\
\hline & PFCm & -3.95 & 16.63 & 9.8612 \\
\hline & Tcv & -17.20 & -23.28 & -8.3536 \\
\hline & PFCcl & -12.11 & 16.48 & 12.1624 \\
\hline & $\mathrm{A} 2$ & -25.35 & -13.60 & 4.5433 \\
\hline & Ссp & -4.23 & -20.99 & 8.4406 \\
\hline & Cca & -3.54 & 4.11 & 13.0190 \\
\hline & Pci & -17.35 & -23.34 & 16.1313 \\
\hline & Pcm & -3.40 & -26.53 & 14.9936 \\
\hline & Pcip & -10.65 & -22.37 & 13.8943 \\
\hline & PCs & -9.43 & -25.73 & 20.5553 \\
\hline & PFCdl & -7.59 & 14.21 & 17.4655 \\
\hline & PMCdl & -7.37 & 3.66 & 20.8523 \\
\hline
\end{tabular}

Table S6: list of regions constituting the rich club for the hemisphere-wise and weighted analysis for the macaque (left hemisphere). The set of regions corresponds to the level $\mathrm{k}$ for which the maximum normalized RCC was observed $(k=32)$. Coordinates correspond to the F99 space. 


\begin{tabular}{|c|c|c|c|c|}
\hline \multirow[b]{2}{*}{ Macaque Right } & \multirow[b]{2}{*}{ RM acronyms } & \multicolumn{3}{|c|}{ Coordinates } \\
\hline & & $\mathbf{x}$ & $y$ & z \\
\hline & Tcpol & 17.26 & -0.09 & -14.89 \\
\hline & Tcs & 24.27 & -12.91 & 1.72 \\
\hline & PFCoi & 10.95 & 6.96 & -0.90 \\
\hline & PFCom & 3.81 & 11.99 & 1.17 \\
\hline & Tcc & 23.09 & -14.43 & -3.60 \\
\hline & PFCol & 16.78 & 14.86 & 8.70 \\
\hline & Tci & 25.28 & -13.39 & -12.01 \\
\hline & PHC & 13.26 & -20.92 & -7.11 \\
\hline & PMCvl & 22.27 & 3.36 & 6.50 \\
\hline & Ip & 21.36 & -4.81 & -1.11 \\
\hline & PFCpo & 4.08 & 23.23 & 9.88 \\
\hline & PFCvl & 19.31 & 9.24 & 7.95 \\
\hline & $\mathrm{PFCm}$ & 2.39 & 14.97 & 9.80 \\
\hline & PFCcl & 11.41 & 15.55 & 12.98 \\
\hline & Ccp & 3.52 & -21.37 & 9.13 \\
\hline & Cca & 2.46 & 1.66 & 12.84 \\
\hline & Pci & 16.73 & -22.96 & 16.89 \\
\hline & Pcm & 2.23 & -27.53 & 14.57 \\
\hline & PCs & 8.93 & -25.49 & 20.82 \\
\hline & PFCdI & 6.40 & 13.92 & 18.36 \\
\hline & PMCdI & 5.86 & 3.77 & 21.29 \\
\hline \multirow{26}{*}{ Human Right } & Tcpol & 33.20 & -5.64 & -33.90 \\
\hline & Tcs & 55.97 & -31.17 & -5.47 \\
\hline & Amyg & 23.09 & -3.99 & -17.69 \\
\hline & PFCoi & 20.06 & 29.17 & -20.70 \\
\hline & Ia & 39.36 & 12.78 & -14.88 \\
\hline & PFCol & 29.45 & 48.83 & -4.62 \\
\hline & PHC & 21.87 & -34.36 & -11.70 \\
\hline & $\mathrm{G}$ & 34.74 & 24.39 & -3.33 \\
\hline & PMCvl & 47.68 & 19.90 & 11.76 \\
\hline & Ip & 42.84 & -4.10 & 1.42 \\
\hline & $\mathrm{HC}$ & 26.50 & -20.96 & -14.25 \\
\hline & PFCvl & 41.28 & 39.39 & 17.94 \\
\hline & VACd & 28.49 & -84.67 & 18.93 \\
\hline & PFCcl & 25.43 & 40.84 & 34.80 \\
\hline & $\mathrm{A} 2$ & 50.00 & -27.44 & 13.62 \\
\hline & Ccp & 9.92 & -48.93 & 26.34 \\
\hline & Cca & 6.01 & 13.65 & 30.62 \\
\hline & S2 & 52.93 & -19.47 & 18.53 \\
\hline & $\mathrm{A} 1$ & 37.34 & -18.91 & 13.51 \\
\hline & M1 & 26.83 & -17.25 & 52.44 \\
\hline & Pci & 41.63 & -53.39 & 42.95 \\
\hline & Pcm & 8.37 & -56.84 & 39.64 \\
\hline & Pcip & 22.69 & -61.91 & 55.96 \\
\hline & PCs & 13.99 & -52.78 & 60.86 \\
\hline & PFCdI & 24.81 & 12.89 & 53.78 \\
\hline & PMCdI & 25.45 & -5.33 & 66.91 \\
\hline
\end{tabular}


Table S7: List of regions constituting the rich club for the hemisphere-wise and weighted analysis for the macaque and human (right hemisphere). The set of regions corresponds to the level $\mathrm{k}$ for which the maximum normalized RCC was observed $(\mathrm{k}=33$ for macaque and $\mathrm{k}=31$ for human). Regions in bold denote rich club regions common in both species. Coordinates for the macaque correspond to the F99 space and for the human to MNI space. 


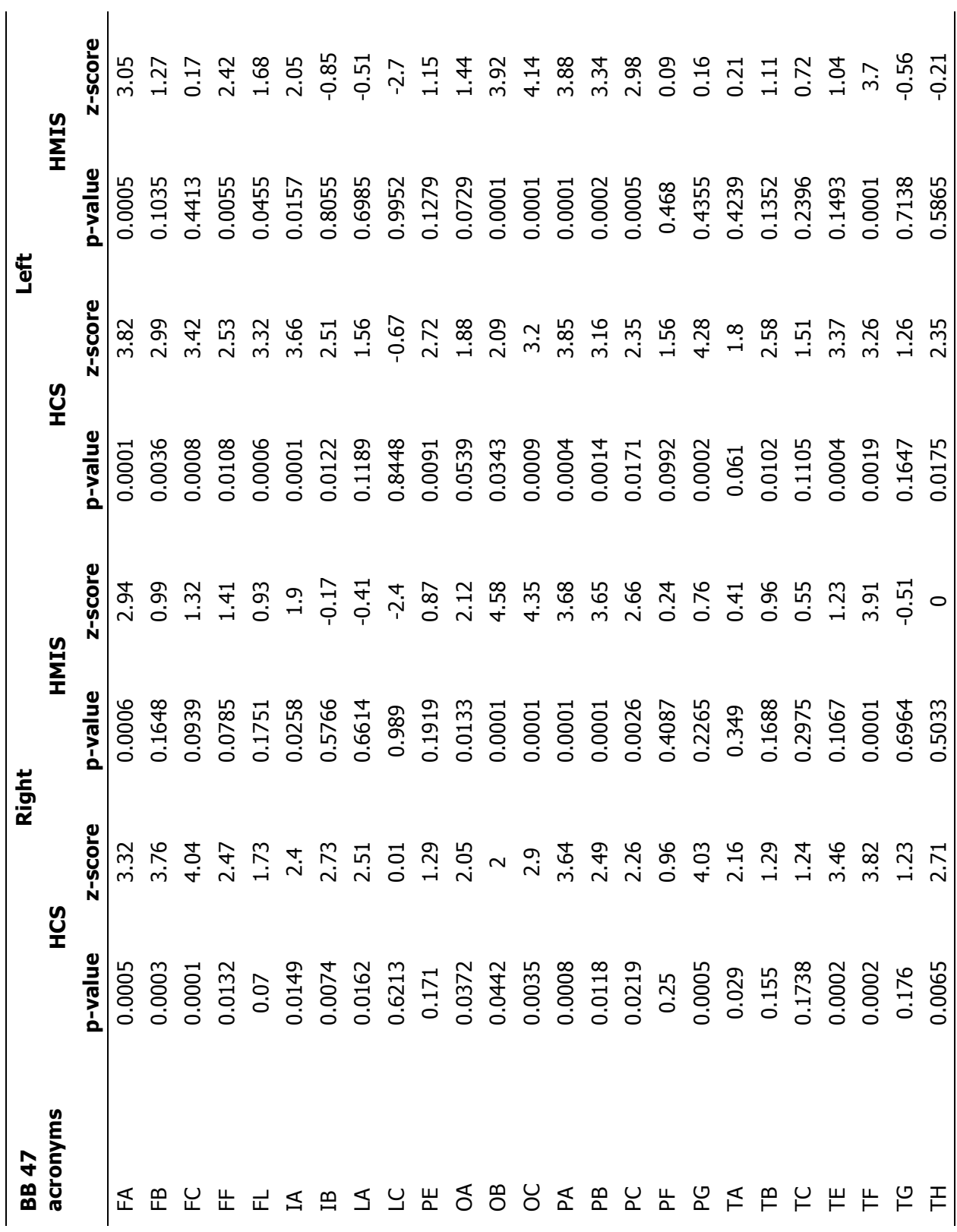

Table S8: HCS and HMIS values for the BB 47 regions for whole brain binary network analysis. The p-values and z-scores of the aforementioned metrics are also depicted. 


\begin{tabular}{|c|c|c|c|c|}
\hline & \multirow[b]{2}{*}{ BB 47 acronyms } & \multicolumn{3}{|c|}{ Coordinates of centre of mass } \\
\hline & & $\mathbf{x}$ & y & $\mathbf{z}$ \\
\hline \multirow[t]{13}{*}{ Macaque } & FB_L & 11.34 & 2.74 & 16.7 \\
\hline & LA_L & 7.98 & 12.82 & 12.49 \\
\hline & LC_L & 3.04 & -15.51 & 13.48 \\
\hline & PE_L & 6.69 & -22.85 & 17.92 \\
\hline & PF_L & 19.64 & -8.85 & 7.25 \\
\hline & PG_L & 16.32 & -22.50 & 15.3 \\
\hline & FB_R & -12.97 & 3.02 & 16.05 \\
\hline & IB_R & -17.68 & 2.73 & -2.72 \\
\hline & LA_R & -9.29 & 13.88 & 12 \\
\hline & LC_R & -3.53 & -12.92 & 13.63 \\
\hline & PE_R & -8.05 & -22.18 & 18.33 \\
\hline & PF_R & -21.08 & -7.84 & 6.18 \\
\hline & PG_R & -17.51 & -22.42 & 14.56 \\
\hline \multirow[t]{10}{*}{ Human } & LA_L & 16.36 & 37.03 & 25.75 \\
\hline & LC_L & 6.07 & -35.91 & 34.74 \\
\hline & PE_L & 11.99 & -53.77 & 51.28 \\
\hline & OA_L & 21.52 & -85.09 & 15.18 \\
\hline & PG_L & 40.26 & -56.97 & 40.15 \\
\hline & LA_R & -18.43 & 37.14 & 25.86 \\
\hline & LC_R & -8.10 & -35.94 & 34.76 \\
\hline & PE_R & -13.95 & -53.8 & 51.24 \\
\hline & PG_R & -42.3 & -56.97 & 40.19 \\
\hline & TB_R & -46.1 & -9.46 & -2.23 \\
\hline
\end{tabular}

Table S9: List of regions constituting the rich club in macaque and human based on the BB 47 parcellation scheme for whole brain binary network analysis. The set of regions corresponds to the level $\mathrm{k}$ for which the maximum normalized RCC was observed (not shown). Regions in bold denote rich club regions common in both species. Coordinates for the macaque correspond to the F99 space and for the human to MNI space. The suffixes $\mathrm{R}$ and $\mathrm{L}$ denote the right and left hemisphere. 


\section{Chapter 3}

\%The script compares the connectivity patterns of a11 nodes in A \%and B. \%This is a cross-matrix variation of the "classic" matching index and \%thus follows the same rationale.

$\%$

\%Input:

\%A, B: NXN binary symmetric connectivity (adjacency) matrices.

\%mode: 1=compute region-to-region cross-matrix MI

$\% \quad 2=$ compute region to al1 region cross matrix MI

\%computationmode:1=calculate MI as intersection/overlap of

$\%$

connections

$\% \quad 2=$ calculate MI as in BCT: common connections/total

$\%$ number of connections*2 (so it scales to 1 as max

$\%$ value)

\%output:

\%CMI A vector of cross matrix MIs (if mode=1)

$\%$ A NXN matrix of cross matrix MIs (if mode=2)

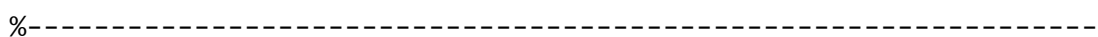

function $\mathrm{CMMI}=$ CrossMatrixMI (A, B, mode, computationmode)

if $($ mode $==1)$

CMMI=zeros $(1$ ength $(\mathrm{A}), 1)$;

for $i=1$ : 1ength (A)

if (computationmode $==1$ )

$\operatorname{CMMI}(i, 1)=1$ ength $($ find $((A(i,:)+B(i,:))==2)) / 1$ ength $($ find $((A(i,:)+B(i,:)) \sim=0$ ) ) ;

else

$\operatorname{CMMI}(i, 1)=2 *(1$ ength $($ find $((A(i,:)+B(i,:))==2)) /(\operatorname{sum}(A(i,:))+\operatorname{sum}(B(i,:)))) ;$ end

end

else

CMMI=zeros $($ length $(A)$, length $(A))$;

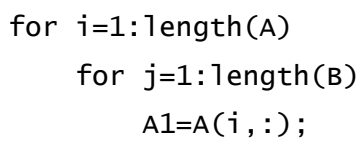


$\mathrm{B} 1=\mathrm{B}(\mathrm{j},:)$;

$A 1\left(\left[\begin{array}{ll}i & j\end{array}\right]\right)=0$;\% Wipe out direct connections between regions for \%which we compute the cross-matrix MI.

B1 $\left(\left[\begin{array}{ll}i & j\end{array}\right]\right)=0$;

if (computationmode $==1$ )

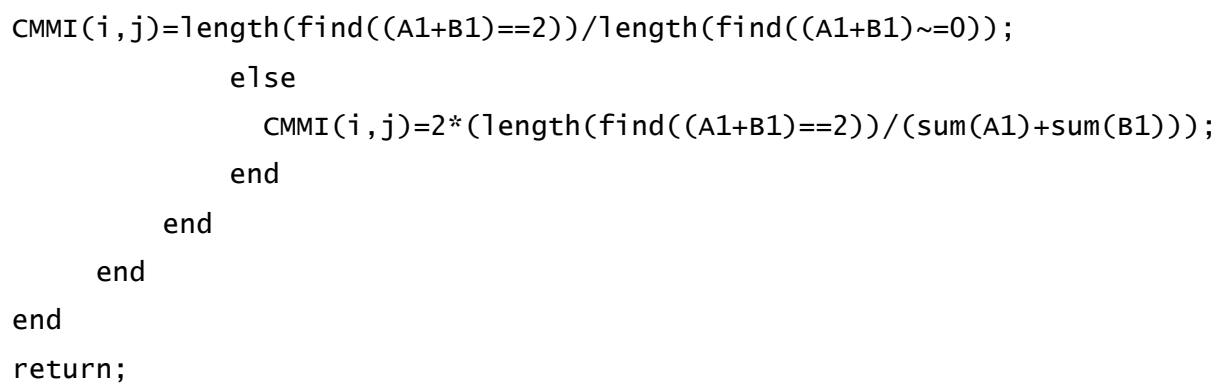

Software S1: MATLAB code for calculating the HCS index. 
Chapter 3 


\section{Chapter 4}

\section{Automatic classification of human cortical layers and areas using high resolution diffusion MRI}

Corresponding publication:

Bastiani, M., Oros-Peusquens, A-M., Seehaus, A., Brenner, D., Moellenhoff, K., Celik, A., Felder, J., Bratzke, H., Shah, N.J., Galuske, R., Goebel, R. and Roebroeck, A. (under review). Automatic classification of human cortical layers and areas using high resolution diffusion MRI. 


\section{Abstract}

The cytoarchitecture and myeloarchitecture of the mammalian cortex is traditionally investigated with histological staining and light microscopy techniques. Recently, several magnetic resonance imaging (MRI) contrast mechanisms have been shown to distinguish cortical substructure corresponding to selected cortical layers, most strongly correlated to myeloarchitecture. However, automatic computer classification of all or most cortical layers over large expanses of cortex and demarcation of distinct cortical areas from MRI has so far remained out of reach. Here, we show that cortical layer and area differentiation can be automatized by unsupervised clustering of high resolution diffusion MRI data. We validate these results by histological analysis of the same tissue. Layers $1,2 / 3,4$ and $5 / 6$ could be reproducibly distinguished in human primary motor and premotor cortex. Beyond this, we show that the signature of diffusion MRI signals over cortical depth can be used to reliably detect area boundaries, where layer characteristics change. These results demonstrate that diffusion MRI can probe layer-specific intracortical fiber organization and contains enough information to automatically classify architecturally distinct cortical areas. 


\section{Introduction}

Historically, there are two main ways to describe the architecture of the mammalian cortex using histology: cytoarchitecture based on counts and morphology of cell bodies (Brodmann, 1909; von Economo and Koskinas, 1925) and myeloarchitecture based on density and orientation of myelinated neurites (Vogt, 1910). Both approaches show a layered organization of the human cerebral cortex, though myeloarchitecture often shows more elaborate sub-layering when compared to the canonical six cytoarchitectural layers of neocortex (Vogt, 1910). There has been a century-old dominance of the cytoarchitectonic classification of human cortex, mostly based on Brodmann's cortical parcellation scheme (Brodmann, 1909), although other schemes are available (Nieuwenhuys, 2013; Vogt, 1910) and new classifications are emerging, for instance based on neurotransmitter receptor architecture (Amunts et al., 2010). Currently, there is a renewed appreciation for the richness of intra-cortical detail visible in its myeoloarchitecture (Nieuwenhuys, 2013). This renewed interest is driven in part by clear indications that several magnetic resonance imaging (MRI) contrasts (e.g. T1 and T2*) in gray matter are dependent on myelin content (Barazany and Assaf, 2012; Duyn et al., 2007; Fatterpekar et al., 2002; Geyer et al., 2011; Lee et al., 2012), which has ignited ambitions of MRI-based histology, possibly even in vivo (Deistung et al., 2013; Dick et al., 2012; Sereno et al., 2013). Diffusion magnetic resonance imaging (dMRI), a technique mostly used to probe brain white matter, has recently also started to be used to highlight different layers in human cortical gray matter (Bastiani et al., 2013; Kleinnijenhuis et al., 2012; Leuze et al., 2012; Oros-Peusquens et al., 2012; Roebroeck et al., 2012). This suggests layered intracortical microcircuitry can be probed, and cortical layers can be delineated, based on the average organization of local neurite orientation.

Here we show that high-resolution dMRI data acquired post mortem can be used to derive two separate, but related, aspects of human cortical gray matter microstructure. First, we distinguish the 
majority of cortical layers over large expanses of neocortex and, second, we demarcate cortical motor and premotor areas corresponding to cytoand myeloarchitectural classifications. The area and layer classifications in this study are achieved by automatic clustering of their diffusion characteristics and are validated by diligent histological analysis of the same tissue.

\section{Materials and Methods}

\section{Tissue preparation}

This study was performed on a block of human brain tissue $(38.94 \times 36.3$ x $23.76 \mathrm{~mm}$ ) which comprised parts of primary motor and medial and lateral premotor cortex. The tissue was obtained 6 hours post mortem from the left hemisphere of a female subject, aged 38, without known neurological or psychiatric disorders. All procedures were approved by the ethical committee of the University Clinic, Frankfurt/M, Germany. The tissue was prepared and fixed for 48 hours using a solution containing $2.6 \%$ paraformaldehyde, $0.8 \%$ iodoacetic acid, $0.8 \%$ sodium periodate, and 0.1 M D - L -lysine in $0.1 \mathrm{M}$ phosphate buffer at $\mathrm{pH} 7.4$ at $4^{\circ} \mathrm{C}$. Thereafter, the tissue was stored in a solution containing $2 \%$ paraformaldehyde in $0,1 \mathrm{M}$ phosphate buffer at $\mathrm{pH} 7.4$ at $4^{\circ} \mathrm{C}$. MR scans were performed after about 1 year of fixation. The tissue was scanned immersed in the fixation solution to ensure long-term preservation for subsequent histological processing (Fig. S1).

MR data acquisition

Measurements for dataset 1 were performed at room temperature on a

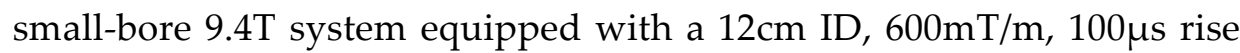
time gradient coil and interfaced to a Siemens Tim Trio console. A $7 \mathrm{~cm}$ loop coil was used for RF transmission and signal reception. A 2D spinecho sequence was modified to include a diffusion preparation module and implement pulsed gradient spin echo (PGSE or Stejskal-Tanner) 
diffusion MR imaging. The measurement parameters included: FOV $53 \times 60 \mathrm{~mm}^{2}$, matrix 156x176, 97 contiguous slices (achieving isotropic resolution of $\left.340 \mu \mathrm{m}^{3}\right), \mathrm{TR}=10000 \mathrm{~ms}, \mathrm{TE}=45 \mathrm{~ms}, \Delta=22.5 \mathrm{~ms}, \delta=3 \mathrm{~ms}$, $|\mathrm{G}|=466 \mathrm{mT} / \mathrm{m}$, flip angle $=90^{\circ}, 4$ averages, $b=3000 \mathrm{~mm}^{-2} \mathrm{~s}, 60$ diffusion encoding directions (obtained by an electrostatic repulsion algorithm on the whole sphere) and six $b=0$ acquisitions. Data acquisition was repeated after one week to test the reproducibility of the results and their stability under different temperatures, and thus different diffusion conditions. Therefore, during the second scanning session (dataset 2), the temperature in the scanner was raised to $30^{\circ} \mathrm{C}$ using an in-bore hot air animal warming system and constantly monitored with a temperature probe. All other acquisition parameters for dataset 2 were the same.

\section{Diffusion MRI data analysis}

Diffusion weighted datasets 1 and 2 were preprocessed in order to correct for image shift and geometric distortions arising from eddy currents induced by diffusion gradients using the FMRIB's Diffusion Toolbox available in FSL (Jenkinson et al., 2012). The estimated transformation matrices were used to rotate the diffusion gradient directions accordingly (Leemans and Jones, 2009) and perform corrections for the corresponding signal magnitude by a normalization using the determinant of the Jacobian matrix. Manual segmentation of the averaged non-diffusion-weighted (i.e. pure T2-weighted or b0) volumes was performed to obtain white and gray matter masks. Affine registration (12 degrees of freedom) from dataset 1 to dataset 2 was performed using the FLIRT toolbox available in FSL (40). Diffusion tensors (DTs) were fitted to the acquired data by linear regression using a least-square minimization approach. Mean diffusivity (MD) maps where obtained computing the average of the three DT eigenvalues in each voxel (Figs. S2). The SNR of the two datasets was calculated as the mean divided by the standard deviation of the b0 signal in each voxel. Mean SNRs were 27.2 and 32.7, for dataset 1 and dataset 2 respectively, 
with a standard deviation of 12 and 13.2. For white matter, the mean SNR was $19.4(\mathrm{std}=6.7)$ and $24.2(\mathrm{std}=8.1)$ in the two datasets respectively. For gray matter, the mean SNR was of $33.5(\mathrm{std}=11.7)$ and 41.6 ( $\mathrm{std}=11.6)$ in the two datasets respectively. The fitting of the standard DT model was evaluated computing the sum of squared error (SSE) at every voxel of the sampled tissue. For white matter the average SSE was $0.24(\operatorname{std}=0.15)$ and $0.73(\operatorname{std}=0.29)$ for the two datasets respectively. For gray matter the average SSE was $0.33(\mathrm{std}=0.23)$ and $0.98(\mathrm{std}=0.57)$ for the two datasets respectively, showing a better fit for dataset 1 .

\section{Histology}

After scanning, the block was cut in half, with the cutting plane parallel to the xy plane of the scan. The anterior part was sectioned at a slice thickness of $60 \mu \mathrm{m}$ using a microtome (Supercut 2050, Reichert-Jung) equipped with a freezing stage (Frigomobil, Leica Microsystems, Wetzlar, Germany). In order to improve orientation within the sliced tissue material later on, blockface photos were taken of every second slice, as in (Choe et al., 2011). This procedure resulted in 343 sections, from which every $5^{\text {th }}$ was stained for myelin using the Gallyas method (Gallyas, 1979), giving 69 myelin stained sections for analysis. Furthermore, every $20^{\text {th }}$ slice was stained for cell bodies with cresyl violet to allow for an unequivocal laminar and areal classification. The sections stained for myelin were digitized using a high-resolution microscope setup (AxioImager Z1, Oberkochen, Germany) equipped with a motorized stage and high resolution camera (Axiocam HRm, Carl Zeiss AG, Oberkochen, Germany). Images were obtained at 50x magnification and assembled using the MosaiX recording technique (Carl Zeiss AG, Oberkochen, Germany). The resulting images were monochrome at 8 bit depth and a pixel resolution of $1.3 \mu \mathrm{m}$. Based on the anatomical descriptions provided by von Economo and Koskinas (von Economo and Koskinas, 1925) and Sanides (Sanides, 1962), the analyzed tissue was identified as primary motor and lateral premotor 
cortex on the basis of its cytoarchitecture. Likewise, the analysis of the myelin stained sections confirmed our cytoarchitectonic classification using the criteria provided by Nieuwenhuys (Nieuwenhuys, 2013) and Vogt (Vogt, 1910). Subsequently cortical layers were delineated on both a cytoarchitectural and myeloarchitectural basis. To align digitized 2D histological sections with the 3D dMRI data, the alignment procedure described in (Seehaus et al., 2013) was used.

\section{Cortical layer demarcation}

The cortical surface and cortical depth were sampled by surface reconstruction of the white/gray matter and pial boundary and discretizing cortical depth in steps between these. Gray matter volume was sampled by nine different meshes of 60000 vertices each at fixed local cortical depth steps $(10-90 \%, 10 \%$ step size) between the white / gray matter boundary (0\%) and the pial / CSF boundary (100\%). The outer white / gray matter and the pial CSF boundaries themselves were avoided to avoid partial volume effects with white matter tissue and embedding fluid. The depth sampling technique is based on Laplace's equation (Jones et al., 2000; Zimmermann et al., 2011) as implemented in BrainVoyager QX (De Martino et al., 2013; Goebel et al., 2006). This technique initially sets two different voltage values at the aforementioned boundaries and computes the smoothed transitional voltages between them. As a result of this initialization step, voltage gradients can be calculated in every voxel of the entire gray matter volume. Integrating across this gradient vector field results in the definition of streamlines which can be used for cortical depth sampling.

Features used to classify cortical layers were derived from the apparent diffusion coefficient (ADC) profile. Samples over a whole sphere of the ADC were obtained from the high angular resolution imaging (HARDI) acquisitions. The ADC profile can be computed as follows: 


$$
A D C_{i}=-\frac{1}{b} \log \left(\frac{S_{i}}{S_{0}}\right)
$$

where $A D C_{i}$ is the coefficient calculated for the $i^{\text {th }}$ diffusion gradient direction, $b$ is the $b$-value in $s^{*} \mathrm{~mm}^{-2}, S_{i}$ is the measured signal when applying the $\mathrm{i}^{\text {th }}$ diffusion encoding gradient and $\mathrm{S}_{0}$ is the b0 signal without diffusion weighting. A $6^{\text {th }}$ order spherical harmonic basis was fitted to the ADC profile at each point of the 9 GM surfaces, applying trilinear interpolation in each diffusion-weighted image. The spherical harmonic basis comprised only even terms as diffusion is modeled as having an equal contribution along opposite directions (therefore we prefer to talk about orientations). Local surface normals, estimated at every reconstructed surface point, where then used to reconstruct radial cortical depth profiles. Each ADC profile was then interpreted in the local 'cortical coordinate system' (cf. (McNab et al., 2013)) by rotating the cortical radial orientation to the z-axis. In this coordinate system four (groups of) feature(s) were derived (Nagy et al., 2013): i) the average of the entire spherical ADC profile (total cortical diffusivity, 1 feature), ii) the value of the ADC profile along the local surface normal (radial cortical diffusivity, 1 feature), iii) the average of the ADC profile over the cortical tangential plane (tangential cortical diffusivity, 1 feature) and iv) the even spherical harmonics coefficients of the ADC profile (28 features). A feature space containing these indices was constructed, resulting into a 60000 (vertices) x 9 (surfaces) x 31 (features). For the voxel-by-voxel layer clustering this space was transformed into a $\left(60000^{*}\right) \times 31$ feature matrix and then fed into an unsupervised k-means clustering algorithm to classify cortical locations into different cortical layers. The clustering algorithm was implemented in the statistics toolbox in Matlab (R2010b, The MathWorks, Natick, Massachusetts, USA) and it tries to cluster each point in the multifeature space around $k$ centroids by minimizing squared Euclidian distances between each point and the proposed centroids. 
The number of clusters (i.e. centroids) $k$ to be identified was set on the basis of a silhouette index analysis, which was implemented in the statistics toolbox in Matlab (R2010b, The MathWorks, Natick, Massachusetts, USA). The silhouette index is a measure of how well a certain element lies in cluster $x$ (Rousseeuw, 1987). It is calculated using the following equation:

$$
s(i)=\frac{b(i)-a(i)}{\max \{a(i), b(i)\}}
$$

where $a(i)$ is the average dissimilarity of element $i$ with the other elements within the same cluster and $b(i)$ is the lowest average dissimilarity of element $\mathrm{i}$ between the other clusters which do not contain i. The silhouette index ranges from -1 (the point was badly assigned to the cluster) to +1 (the element lies in the appropriate cluster and it is very distant from any neighboring cluster). The average silhouette values were computed for all the vertices and setting the number of $k$ centroids from 2 to 7 . The two local maxima ( 3 and 6 ) for dataset 1 were chosen as number of clusters for the k-means algorithm (Fig S3). Qualitatively inspecting the results showed that three clusters corresponded to two cortical layer clusters (in gray matter) and one noise cluster (almost invariably located at tissue boundaries) and similarly six clusters corresponded to four cortical layer clusters in gray matter and one noise cluster. Therefore, in the main text, we refer to these results as the two-layer cluster and four-layer cluster results, respectively. Since the centroid locations are always initialized at random positions, the clustering algorithm was run 100 times and the best solution (i.e. the one which minimized the sum of differences between the feature space and the centroids) was kept. No spatial proximity information was used in the feature matrix for the layerclustering procedure, i.e. it was based on absolute (in the total cortical 
diffusivity) and orientation dependent diffusion information (in the other features) only.

For visualization purposes, the layer cluster maps were interpolated using mode filtering. First, the original layer cluster maps was interpolated to three times the resolution using a nearest neighbour interpolation method as implemented in Matlab (R2010b, The MathWorks, Natick, Massachusetts, USA). Then, in the interpolated volume, every element was substituted with the mode of the values contained in a $5 \times 5 \times 5$ window.

To assess reproducibility between the two datasets and correspondence between histology and dMRI-based layer clustering, cross-table contingency analysis and Chi-Squared statistics were used to evaluate its significance, as implemented in Matlab (R2010b, The MathWorks, Natick, Massachusetts, USA). After myelo- and cytoarchitectural layers were segmented by a skilled anatomist, the corresponding layer number was assigned to every pixel of the histological section. Then, the resolution of the histological sections was downsampled to match the one of the dMRI datasets ( $340 \mu \mathrm{m}$ isotropic), by using a nearest-neighbor method as implemented in Matlab (R2010b, The MathWorks, Natick, Massachusetts, USA). This was done in order to get an appropriate number of degrees of freedom for Chi-Square statistics when performing the cross-table contingency analysis.

\section{Cortical area demarcation}

The implementation of the area boundary demarcation technique used ADC-based indices defined across cortical depth and a sampling grid defined in the 3 dimensional space which comprised the tissue sample. The approach is based on (Schleicher et al., 2000) and used a local stepping procedure based on Mahalanobis distances, extended to a 3D cortical sampling grid. To implement the area demarcation technique, a new grid was defined in gray matter. The three dimensional grid samples the cortical volume both radially at 9 equi-spaced depths and 
tangentially in the latero-medial and antero-posterior direction in 120 $\mu \mathrm{m}$ steps. This approach is capable of reliably following folded cortex at different relative proportional depths (of $0-100 \%$, see above). Furthermore, the streamlines which connect the two outer cortical boundaries connect corresponding points within the depth sampling grids across multiple cortex depth planes, forming what we refer to as cortical depth profiles (Fig. S6). The $100 \times 200 \times 9$ three dimensional grid covered the crown of the precentral gyrus and the precentral sulcus. The grid was formed by laying down a 200 discrete point streamline over the surface reconstruction in the anterio-posterior direction. This streamline was then replicated in the latero-medial direction 99 times, at $170 \mu \mathrm{m}$ spacing and in the cortical depth direction over the 9 surfaces. To increase the sensitivity of the boundary algorithm and reduce artefacts mainly caused by local blood vessels within cortical gray matter, the ADC-based indices were averaged across 10 latero-medial grid positions, resulting in a $10 \times 200 \times 9$ three-dimensional grid. The areal classification was performed independently of the layer classification on a single diffusion information vector per cortical surface location, which contains all diffusion feature information along cortical depth. The feature space consisted of the first four (i.e. $1^{\text {st }}, 2^{\text {nd }}, 3^{\text {rd }}$ and $4^{\text {th }}$ order) moments around the mean of each of the ADC indices (total, radial and tangential) calculated along the cortical depth profile, using the sampled grid. Therefore, 12 different coefficients were defined at every grid point, forming a local coefficient profile. Stepping along the antero-posterior direction, the Mahalanobis distance between profiles in two blocks of $\mathrm{N}$ contiguous profiles was computed. To compute the Mahalanobis distance between two parcels, the following equation was used:

$$
D=\left(\overline{X_{1}}-\overline{X_{2}}\right) C^{-1}\left(\overline{X_{1}}-\overline{X_{2}}\right)^{\prime}
$$


where $\bar{X}_{1}$ and $\bar{X}_{2}$ are the mean feature vectors of every block at each integration step and $C$ is the pooled covariance matrix (Amunts et al., 2010). The block size $\mathrm{N}$ was varied between 12 to 24 profiles in each block to ensure stability of the pooled covariance matrix and reproducibility of the identified boundary over spatial scales. Significant boundaries were identified using Hotelling's $\mathrm{T}^{2}$ statistics corrected for multiple comparisons using Bonferroni correction. Finally, the total number of significant counts was computed, summing the number of significant candidate boundaries at each profile block size (spatial scale; see Fig. 4, lower left inset), across the ten different averaged lattices. These total number of counts were thresholded at 4.5 standard deviations (red lines in Fig. 4) to demarcate area boundaries in both datasets.

\section{Results}

We acquired dMRI data from a human post-mortem brain tissue sample, comprising parts of primary motor and medial and lateral premotor cortex (Fig. 1) on a high-field (9.4T), small-bore MRI system. We were able to demarcate cortical lamination patterns over large expanses of human motor cortex by applying a clustering algorithm to very high-resolution dMRI signal characteristics. Crucially, the diffusion characteristics were derived from high isotropic spatial resolution (340 $\mu \mathrm{m})$ and high angular resolution dMRI data which is rotated into the local cortical coordinate frame comprising radial and tangential orientations to the cortical surface.

\section{Automatic demarcation of cortical layers}

Fig. 1 shows the results of the automated layer classification algorithm applied to the full extent of cortex in the tissue sample, containing motor and premotor areas in precentral and superior frontal gyri. For reproducibility of the results two separate datasets were acquired on the same tissue block (dataset 1 and dataset 2) one week apart with identical 
acquisition parameters but at different temperatures, and thus under different diffusion conditions. The classification results for the two different datasets show a very high degree of correspondence, indicating strong reproducibility. Silhouette analysis (Fig. S3) for the optimal number of clusters in the grey matter gave two local optima, the first for two layer clusters and the second for four layer clusters. The upper row in panels $\mathrm{A}, \mathrm{B}$ and $\mathrm{C}$ shows the two layer cluster (twocluster) result, whereas the lower row shows the four layer cluster (fourcluster) result. It can be seen that the two-cluster result clearly identifies a boundary between superficial and deep cortical layers in a reproducible way between dataset 1 and 2 .
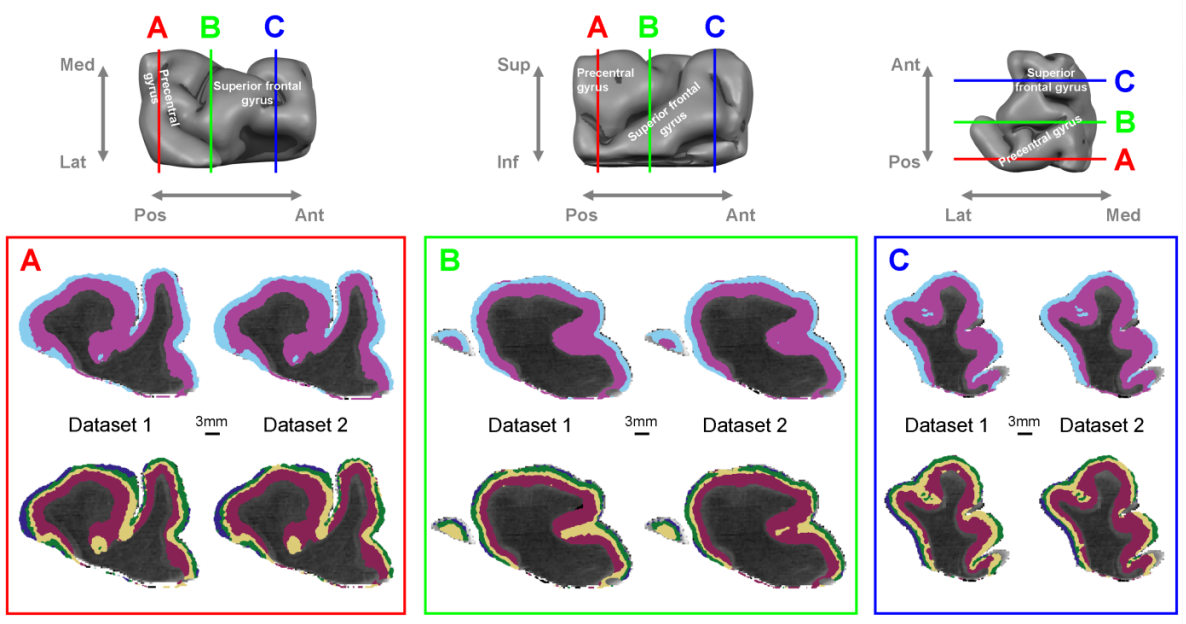

Figure 1: Automatic layer classification from high-resolution dMRI for dataset 1 and 2. Top row: macro-anatomical description of the tissue sample and virtual section plane locations. Bottom row $(\mathrm{A}, \mathrm{B}, \mathrm{C})$ : coronal sections (viewed from the anterior side) through the $3 \mathrm{D}$ dMRI data showing automated cortical layer classification results overlaid on mean diffusivity (MD) maps. Top row within the three panels is the two-layer cluster result, bottom row is the four-layer cluster result.

In the selected slices, the classification algorithm marks twoclusters maintaining a consistent boundary and their relative depth 
ordering over most of the studied expanse of cortex. This is an important finding, given that no information on spatial cortical location (such as cortical depth or tangential proximity to other voxels) was given to the algorithm; it operated only on orientation dependent diffusion characteristics in the local cortical coordinate frame. Note that the light blue superficial band disappears in some locations because of the shallow or highly oblique slicing of gyral/sulcal walls in the depicted section plane. The four-cluster result in the lower row shows that the information in the diffusion data supports even further subdivision into four different clusters in many parts of the cortical ribbon. To quantitatively investigate the reproducibility between datasets, we performed cross-table contingency analysis and Chi-Squared statistics. Cross-table contingency analysis between the two- and the four-cluster results for the same datasets showed that the four-cluster result subdivides each of the two classes from the two-cluster results, retaining the two-cluster boundary (see Fig. S4). Again, these clusters maintain their relative depth ordering almost everywhere. While only a subset of the clusters is present at certain locations, most gray matter that is nearly perpendicularly cut by the section planes is subdivided into four layer clusters with only the outermost and thinnest (dark blue) layer cluster sometimes missing identification. Automatically labeled clusters broaden and narrow depending on their location particularly in gyral crowns or sulcal fundi, reflecting known anatomy (Bok, 1929).

The reproducibility of the layer classification result between the two separate datasets is shown in Fig. 2 (see also tables S1 and S2). The correspondence (in terms of the cross-table fraction) of the spatial clustering results between dataset 1 and dataset 2 is larger than 0.94 (mean $=0.97$ ) for the two layer cluster analysis and larger than 0.79 (mean $=0.86)$ for the four layer cluster analysis. The Chi-Squared contingency analysis showed a highly significant association between the two datasets for both the two layer cluster result $\left(\chi^{2}=5.18 \mathrm{e}+005, \mathrm{p}<\right.$ $0.001)$ and the four layer cluster result $\left(\chi^{2}=7.78 \mathrm{e}+005, \mathrm{p}<0.001\right)$. The 
degree of similarity of the signal profile (rotated to the cortical frame) between the two datasets for corresponding clusters is very high with correlations of 0.95 and 0.98 for the two layer cluster analysis result and between 0.87 and 0.99 for the four layer cluster analysis. This shows that the distinctiveness of the signal profile, which leads to the identification of the different layer clusters, is also highly reproducible.
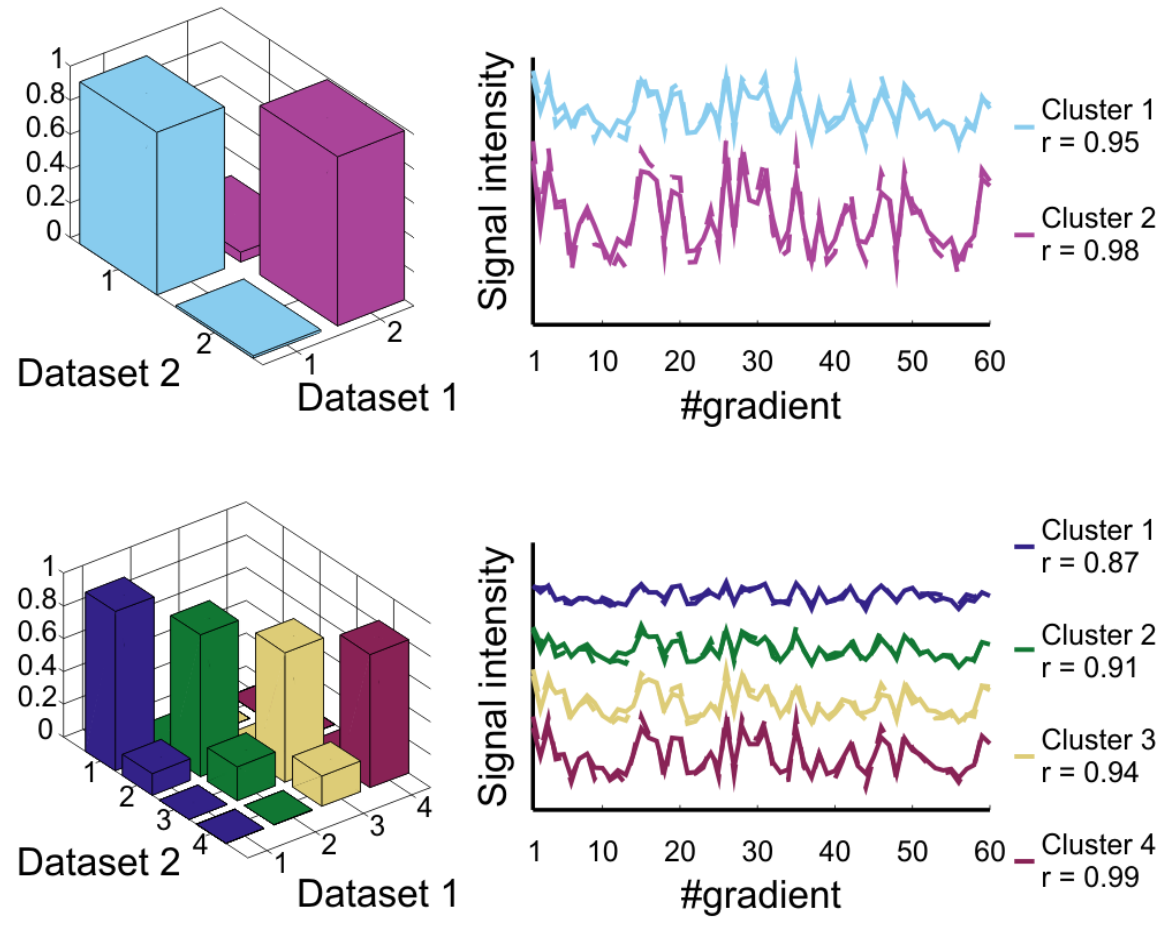

Figure 2: Quantitative reproducibility analysis of the two-layer (top row) and the fourlayer result (bottom row). Left column: cross-table analysis of dataset 1 against dataset 2, right column correlation analysis of the layer cluster signal profile between dataset 1 (solid line) and dataset 2 (dashed line). 

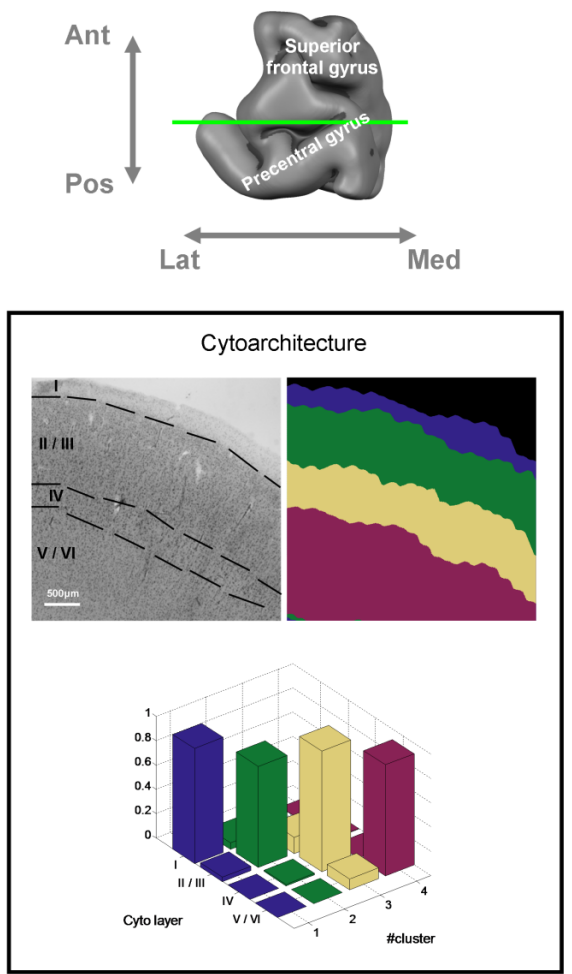
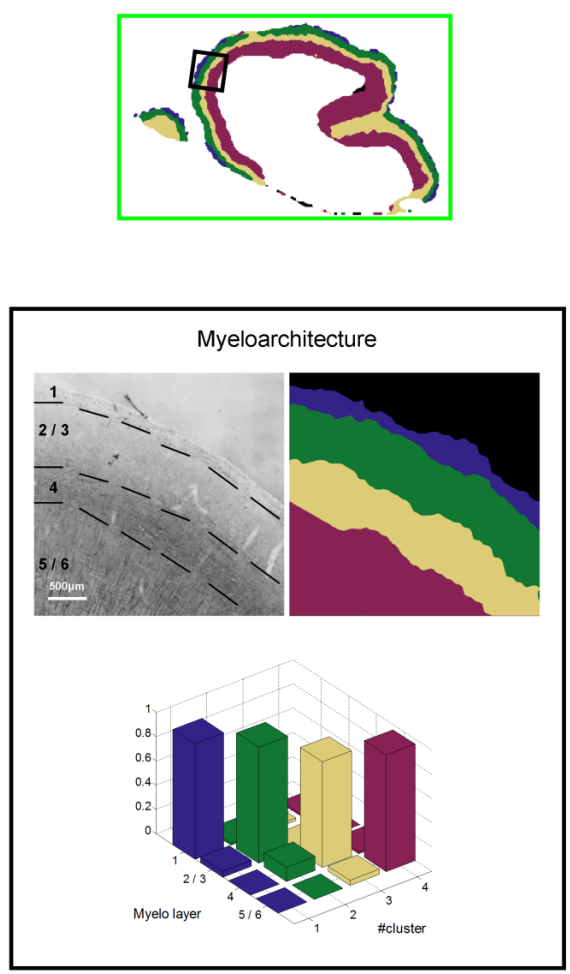

Figure 3: Correspondence between the four layer cluster result of dataset 1 and histology on the same tissue block. Upper row, location of the coronal section. Lower panels, correspondence to cytoarchitecture (left) and myeloarchitecture (right). For each panel, the upper row depicts histological classification of layers and dMRI layer cluster result, and bottom row shows the cross-table contingency analysis between histology and $\mathrm{dMRI}$ based layer clustering.

\section{Histological validation of automated layer clustering}

We validated our automatic classification finding by histological sectioning and staining of the same tissue sample, as shown in Fig. 3. The laminar organization obtained from the layer classification is shown along with cytoarchitectural and myeloarchitectural classifications of the same tissue. Quantitative correspondence between automated clustering and histology is again reported by cross-table contingency analysis. A 
very high correspondence is found between both cytoarchitecture and myeloarchitecture and automatic clustering based on dMRI. For myeloarchitecture classification when grouping together both layers 2 and 3 and layers 5 and 6 into two-layer complexes the correspondence is larger than 0.87 (mean $\left.=0.93, \chi^{2}=127.02, p<0.001\right)$. For cytoarchitecture, again when grouping together layers II and III and layers V and VI, the correspondence is larger than 0.83 (mean $=0.92, \chi^{2}=110.22, p<0.001$ ). This shows there is a clear match between the layer boundaries identified by the algorithm and both the myeloarchitectural and the cytoarchitectural ones identified in the stained sections. As we expect dMRI signal to be more sensitive to myelinated neurites, we use roman numerals to label cortical layers in the following. A detailed analysis of the architectural correspondence reveals layer cluster 1 to correspond to cortical layer 1, cluster 2 to layers $2 / 3$, cluster 3 to layer 4 and, finally, cluster 4 to layers 5/6. That is, layers 1 and 4 are quite consistently identified as an individual cluster. Cortical layers 2 and 3 and cortical layers 5 and 6 are each clustered together. This result could be robustly reproduced in another part of the tissue sample (see Fig. S5).

\section{Automatic boundary detection of cortical areas}

Having identified cortical lamination patterns, we tested whether we were also able to architecturally demarcate different cortical areas from dMRI data in gray matter, based on abrupt changes in lamination. Indeed, Fig 4. shows that the signature of dMRI signals over cortical depth can be used to reliably detect cortical area boundaries. The dMRIbased demarcation is based on local Mahalanobis distances between feature vectors defined over cortical depth (See Fig. S6). In this approach we move along a three-dimensional intra-cortical grid (with one depth dimension and two tangential dimensions) from posterior to anterior along the precentral gyrus and sulcus (Fig. 4, upper left), collecting information on local feature vector distances. The points of the largest local feature vector distances over multiple window sizes (spatial scales) identify cortical area boundaries (Fig. 4, lower left). Two boundaries are 
most prominent and consistently identified in both datasets. The first is close to the crown of the precentral gyrus, the second is located in the fundus of the precentral sulcus (Fig. 4, right panels). When observing the automatic classification of cortical layers at these boundaries, relatively abrupt local changes in layering can be seen as the underlying cause for boundary detection (insets in Fig. 4). At the boundary located more posteriorly, on the crown of the precentral gyrus, we observe a widening of layer cluster 4 , corresponding to the deep layers 5 and 6 . This leads to a thinning of the more superficial layers and a neardisappearance of layer 1 . At the boundary located more anteriorly in the precentral sulcus, it is rather cluster 3, corresponding to layer 4, which broadens somewhat abruptly.
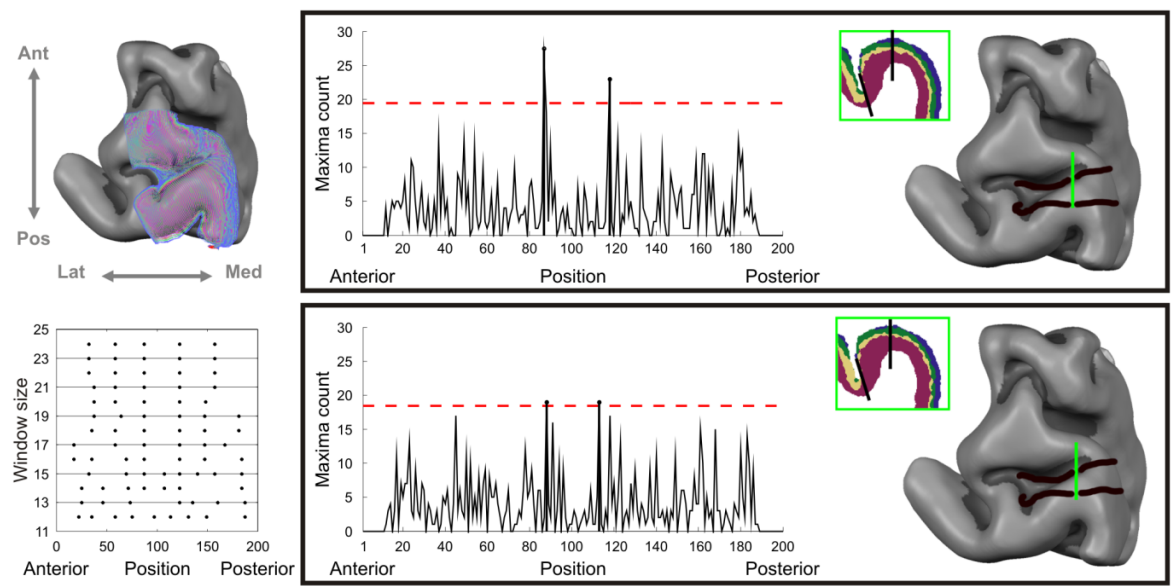

Figure 4: Automated observer independent cortical area boundary detection on dMRI data. Left column, upper inset: cortical depth sampling grid straddling the precentral gyrus and sulcus. Left column, lower inset: thresholded Hotelling's statistics for significant detected boundary over different window sizes and cortical anterio-posterior position. Right panels for dataset 1 (top) and dataset 2 (bottom): on the left the significant boundary index summed over scales with the horizontal line corresponding to 4.5 standard deviations and on the right the identified superthreshold cortical area boundaries on the sampling grid. 


\section{Discussion}

In an in-depth analysis of human post mortem brain tissue we have shown that cortical layer and area differentiation can be automated by unsupervised algorithms applied to high-resolution dMRI data. These results were validated by histological analysis of the same tissue block.

\section{Automatic layer identification}

The two layer cluster result demarcates a clear boundary between superficial and deep cortical layers in a reproducible way. Considering the histological validation results (Fig. 3) there is a very robust identification of what Vogt (Vogt, 1910) already called the inner and outer main cortical zones (the "Innere" and "Äußere Hauptzone"), with the superficial zone containing layers 1,2 and 3 and the deep zone containing layers 4,5 and 6. Beyond that, the four-cluster result subdivides each zone further into layer clusters, corresponding to layer 1 , a layer $2 / 3$ complex, layer 4 and a layer $5 / 6$ complex. Although slightly less consistent than the two-cluster result, the more detailed four-cluster result shows a remarkable consistency over long stretches of cortex and a high reproducibility with cross table correspondence fractions in the 0.8-0.9 range.

As shown in Fig. 1, there were interruptions of some layers (particularly layer 1) along the length of the cortex in the four layer cluster result. The most likely cause of this is partial volume effects since our high spatial resolution of $340 \mu \mathrm{m}$ is still only just sufficient to identify the very thin layer 1, comprising only about 100-200 $\mu \mathrm{m}$. We are still able to identify this structure of about half a voxel width because the neighboring embedding fluid has no orientation dependence (i.e. isotropic diffusion). Thus, adding its signal decreases orientation dependent contrast-to-noise of the tissue contribution but does not destroy the important orientation dependent signal structure in the voxel. Even further increases of spatial resolution can alleviate this issue, and could potentially also help uniquely identify thin deeper 
layers. An interesting challenge is achieving such higher resolution while maintaining, or even extending, spatial tissue coverage and angular diffusion resolution (i.e. number of diffusion encoding directions).

Our histological analysis revealed that the automatic clustering identified layer 1 , layers $2 / 3$, layer 4 and layers $5 / 6$ consistently in the same clusters. In correspondence with the generally accepted six-layer structure of neocortex, we found an almost equally high level of agreement of the automatic dMRI clustering with the cytoarchitectural and myeloarchitectural classification of the cortical layers. Agreement with the dMRI clustering with the myeloarchitecture is qualitatively slightly higher (i.e. marginally higher cross-table fractions) but correspondence for both is very high and statistically significant. Historically, Vogt argued that it is only the sub-classifications of the six layers that can be different and in particularly more detailed in myelothan in cytoarchitecture. In addition, Hellwig (Hellwig, 1993) found that when limited to the six canonical neocortical layers, myeloarchitecture can be predicted from cytoarchitecture, confirming their basic agreement. Because of the basic orientational contrast of dMRI, it is highly likely that the automatic layer classification presented here is mostly sensitive to myeloarchitecture. For instance, layer 1 and 4 are consistently identified because of the strong orientational contrast provided by their characteristic tangential plexus of fibers.

\section{Automatic area demarcation}

The area classification results show that, beyond layer-specific intracortical fiber organization, the exquisite orientation sensitivity of dMRI contains enough information to automatically classify architecturally distinct cortical areas. Two boundaries were reproducibly identified, the first marking a boundary close to the crown of the precentral gyrus, the second a boundary in the fundus of the precentral sulcus. These are very similar to the established motor cortex parcellation originally described by Vogt (Vogt, 1910) and later 
confirmed by Sanides (Sanides, 1962) who divided the precentral and superior frontal gyrus into areas 42, 39 and 38. Indeed, the boundary between areas 42 and 39 lies close to the crown of the precentral gyrus in the superior part of the brain, which is also demarcated by our posteriormost boundary. The boundary between areas 39 and 38 is located close to the fundus of the precentral sulcus, corresponding very well to our anteriormost boundary. Here broadly speaking, area 42 agrees with Brodmann's area 4 and primary motor cortex while 38 and 39 are a further subdivision of Brodmann's area 6 and a part of premotor cortex. Furthermore, the identified boundaries agree well with observer-independent cytoarchitecture (Fischl et al., 2008; Geyer et al., 1996) which was recently also confirmed using in-vivo myelin mapping (Glasser and Van Essen, 2011).

The detection and automatic classification of fine-grained microstructural distinctions between cortical layers and architectural areas establishes dMRI firmly as an important MRI modality in the emerging field of MRI-based histology (Geyer et al., 2011; Nagy et al., 2013; Sereno et al., 2013; Van Essen and Glasser, 2013). It also sets basic resolution requirements on $\mathrm{dMRI}$ data (such as spatial and angular resolution) for the purpose of gray matter classification and paves the way for translating this technique to in-vivo acquisitions. Combination of dMRI with other MR contrasts (Duyn et al., 2007; Fatterpekar et al., 2002; Geyer et al., 2011) and with complementary data in 3D histology atlases (Amunts et al., 2013) will provide an even richer multidimensional feature space to be exploited for layer and area demarcation. This also shows that dMRI has a potential to extend its already important role in the field of human connectomics (Sporns, 2013; Sporns et al., 2005; Van Essen et al., 2013). The layer and area classification results reported here show dMRI data can be used not only of the delineation of white matter fiber tracts (the connectome graph edges) between gray matter areas, but also the cortical areas themselves (the connectome graph nodes). This is especially significant, 
since the accurate demarcation of heterogeneous spatial cortical nodes that are internally homogeneous is an outstanding challenge in human connectomics (Fornito et al., 2013).

\section{Acknowledgments}

We would like to thank Kirsten Wehner for the histological preparation of the tissue, Anna-Lena Keller for help with the histological data acquisition and Andreas Matusch for advice on macro anatomical classification. This work was supported by the European Research Council (ERC) under European Union's Seventh Framework Programme FP7/2007-2013/ERC (Grant Agreement Number 269853). 


\section{References}

Amunts, K., Lenzen, M., Friederici, A.D., Schleicher, A., Morosan, P., PalomeroGallagher, N., Zilles, K., 2010. Broca's region: novel organizational principles and multiple receptor mapping. PLoS Biol 8.

Amunts, K., Lepage, C., Borgeat, L., Mohlberg, H., Dickscheid, T., Rousseau, M.E., Bludau, S., Bazin, P.L., Lewis, L.B., Oros-Peusquens, A.M., Shah, N.J., Lippert, T., Zilles, K., Evans, A.C., 2013. BigBrain: an ultrahigh-resolution 3D human brain model. Science $340,1472-1475$.

Barazany, D., Assaf, Y., 2012. Visualization of cortical lamination patterns with magnetic resonance imaging. Cereb Cortex 22, 2016-2023.

Bastiani, M., Oros-Peusquens, A.M., Brenner, D., Moellenhoff, K.H.M., Seehaus, A.K., Celik, A., Felder, J., Matusch, A., Galuske, R., Bratzke, H., Shah, N.J., Goebel, R., Roebroeck, A., 2013. Cortical Fiber Insertions and Automated Layer Classification in Human Motor Cortex from 9.4T Diffusion MRI. Proceedings of the Joint Annual Meeting of ISMRM-ESMRMB, Salt Lake City, Utah, USA.

Bok, S.T., 1929. Der Einfluss der in den Furchen und Windungen auftretenden Krümmungen der Grosshirnrinde auf die Rindenarchitektur. Z. Gesamte Neurol. Psychiatr. 12, 682-750.

Brodmann, K., 1909. Vergleichende Lokalisationslehre der Grosshirnrinde in ihren Prinzipien dargestellt auf Grund des Zellenbaues, Leipzig.

Choe, A.S., Gao, Y.R., Li, X., Compton, K.B., Stepniewska, I., Anderson, A.W., 2011. Accuracy of image registration between MRI and light microscopy in the ex vivo brain. Magnetic Resonance Imaging 29, 683-692.

De Martino, F., Zimmermann, J., Muckli, L., Ugurbil, K., Yacoub, E., Goebel, R., 2013. Cortical Depth Dependent Functional Responses in Humans at 7T: Improved Specificity with 3D GRASE. PLoS One 8.

Deistung, A., Schafer, A., Schweser, F., Biedermann, U., Turner, R., Reichenbach, J.R., 2013. Toward in vivo histology: a comparison of quantitative susceptibility mapping (QSM) with magnitude-, phase-, and R2*-imaging at ultra-high magnetic field strength. Neuroimage 65, 299-314.

Dick, F., Tierney, A.T., Lutti, A., Josephs, O., Sereno, M.I., Weiskopf, N., 2012. In vivo functional and myeloarchitectonic mapping of human primary auditory areas. J Neurosci 32, 16095-16105.

Duyn, J.H., van Gelderen, P., Li, T.Q., de Zwart, J.A., Koretsky, A.P., Fukunaga, M., 2007. High-field MRI of brain cortical substructure based on signal phase. Proc Natl Acad Sci U S A 104, 11796-11801.

Fatterpekar, G.M., Naidich, T.P., Delman, B.N., Aguinaldo, J.G., Gultekin, S.H., Sherwood, C.C., Hof, P.R., Drayer, B.P., Fayad, Z.A., 2002. Cytoarchitecture of the human cerebral cortex: MR microscopy of excised specimens at 9.4 Tesla. AJNR Am J Neuroradiol 23, 1313-1321. 
Fischl, B., Rajendran, N., Busa, E., Augustinack, J., Hinds, O., Yeo, B.T., Mohlberg, H., Amunts, K., Zilles, K., 2008. Cortical folding patterns and predicting cytoarchitecture. Cereb Cortex 18, 1973-1980.

Fornito, A., Zalesky, A., Breakspear, M., 2013. Graph analysis of the human connectome: promise, progress, and pitfalls. Neuroimage 80, 426-444.

Gallyas, F., 1979. Silver staining of myelin by means of physical development. Neurol Res 1, 203-209.

Geyer, S., Ledberg, A., Schleicher, A., Kinomura, S., Schormann, T., Burgel, U., Klingberg, T., Larsson, J., Zilles, K., Roland, P.E., 1996. Two different areas within the primary motor cortex of man. Nature $382,805-807$.

Geyer, S., Weiss, M., Reimann, K., Lohmann, G., Turner, R., 2011. Microstructural Parcellation of the Human Cerebral Cortex - From Brodmann's Post-Mortem Map to in vivo Mapping with High-Field Magnetic Resonance Imaging. Front Hum Neurosci 5, 19.

Glasser, M.F., Van Essen, D.C., 2011. Mapping human cortical areas in vivo based on myelin content as revealed by T1- and T2-weighted MRI. J Neurosci 31, 11597-11616.

Goebel, R., Esposito, F., Formisano, E., 2006. Analysis of functional image analysis contest (FIAC) data with brainvoyager QX: From single-subject to cortically aligned group general linear model analysis and self-organizing group independent component analysis. Hum Brain Mapp 27, 392-401.

Hellwig, B., 1993. How the myelin picture of the human cerebral cortex can be computed from cytoarchitectural data. A bridge between von Economo and Vogt. J Hirnforsch 34, 387-402.

Jenkinson, M., Beckmann, C.F., Behrens, T.E., Woolrich, M.W., Smith, S.M., 2012. Fsl. Neuroimage 62, 782-790.

Jones, S.E., Buchbinder, B.R., Aharon, I., 2000. Three-dimensional mapping of cortical thickness using Laplace's equation. Hum Brain Mapp 11, 12-32.

Kleinnijenhuis, M., Zerbi, V., Kusters, B., Slump, C.H., Barth, M., van Cappellen van Walsum, A.M., 2012. Layer-specific diffusion weighted imaging in human primary visual cortex in vitro. Cortex.

Lee, J., Shmueli, K., Kang, B.T., Yao, B., Fukunaga, M., van Gelderen, P., Palumbo, S., Bosetti, F., Silva, A.C., Duyn, J.H., 2012. The contribution of myelin to magnetic susceptibility-weighted contrasts in high-field MRI of the brain. Neuroimage 59, 39673975.

Leemans, A., Jones, D.K., 2009. The B-matrix must be rotated when correcting for subject motion in DTI data. Magn Reson Med 61, 1336-1349.

Leuze, C.W., Anwander, A., Bazin, P.L., Dhital, B., Stuber, C., Reimann, K., Geyer, S., Turner, R., 2012. Layer-Specific Intracortical Connectivity Revealed with Diffusion MRI. Cereb Cortex. 
McNab, J.A., Polimeni, J.R., Wang, R., Augustinack, J.C., Fujimoto, K., Stevens, A., Triantafyllou, C., Janssens, T., Farivar, R., Folkerth, R.D., Vanduffel, W., Wald, L.L., 2013. Surface based analysis of diffusion orientation for identifying architectonic domains in the in vivo human cortex. Neuroimage 69, 87-100.

Nagy, Z., Alexander, D.C., Thomas, D.L., Weiskopf, N., Sereno, M.I., 2013. Using high angular resolution diffusion imaging data to discriminate cortical regions. PLoS One 8, e63842.

Nieuwenhuys, R., 2013. The myeloarchitectonic studies on the human cerebral cortex of the Vogt-Vogt school, and their significance for the interpretation of functional neuroimaging data. Brain Struct Funct 218, 303-352.

Oros-Peusquens, A.M., Roebroeck, A., Brenner, D., Moellenhoff, K.H.M., Celik, A., Felder, J., Matusch, A., Galuske, R., Bratzke, H., Shah, N.J., 2012. Cortical layers one by one: diffusion properties at 160um resolution. Proceedings of the Joint Annual Meeting of ISMRM-ESMRMB, Melbourne, Australia.

Roebroeck, A., Oros-Peusquens, A.M., Brenner, D., Moellenhoff, K.H.M., Celik, A., Felder, J., Matusch, A., Galuske, R., Bratzke, H., Shah, N.J., 2012. Human cortical layers detected with high resolution diffusion MRI at 9.4T. Proceedings of the Annual Meeting of OHBM, Beijing, China.

Rousseeuw, P.J., 1987. Silhouettes: A graphical aid to the interpretation and validation of cluster analysis. Journal of Computational and Applied Mathematics 20, 53-65.

Sanides, F., 1962. [Architectonics of the human frontal lobe of the brain. With a demonstration of the principles of its formation as a reflection of phylogenetic differentiation of the cerebral cortex]. Monogr Gesamtgeb Neurol Psychiatr 98, 1-201.

Schleicher, A., Amunts, K., Geyer, S., Kowalski, T., Schormann, T., Palomero-Gallagher, N., Zilles, K., 2000. A stereological approach to human cortical architecture: identification and delineation of cortical areas. J Chem Neuroanat 20, 31-47.

Seehaus, A.K., Roebroeck, A., Chiry, O., Kim, D.S., Ronen, I., Bratzke, H., Goebel, R., Galuske, R.A., 2013. Histological validation of DW-MRI tractography in human postmortem tissue. Cereb Cortex 23, 442-450.

Sereno, M.I., Lutti, A., Weiskopf, N., Dick, F., 2013. Mapping the human cortical surface by combining quantitative T(1) with retinotopy. Cereb Cortex 23, 2261-2268.

Sporns, O., 2013. The human connectome: origins and challenges. Neuroimage 80, 53-61.

Sporns, O., Tononi, G., Kotter, R., 2005. The human connectome: A structural description of the human brain. PLoS Comput Biol 1, e42.

Van Essen, D.C., Glasser, M.F., 2013. In vivo architectonics: A cortico-centric perspective. Neuroimage.

Van Essen, D.C., Smith, S.M., Barch, D.M., Behrens, T.E.J., Yacoub, E., Ugurbil, K., Consortium, W.-M.H., 2013. The WU-Minn Human Connectome Project: An overview. Neuroimage 80, 62-79. 
Vogt, O., 1910. Die myeloarchitektonische felderung des menschlichen stirnhirns. J Psychol Neurol 15, 221-232.

von Economo, C., Koskinas, G.N., 1925. Die Cytoarchitektonik der Hirnrinde des erwachsenen Menschen. Julius Springer, Vienna.

Zimmermann, J., Goebel, R., De Martino, F., van de Moortele, P.F., Feinberg, D., Adriany, G., Chaimow, D., Shmuel, A., Ugurbil, K., Yacoub, E., 2011. Mapping the organization of axis of motion selective features in human area MT using high-field fMRI. PLoS One 6, e28716. 


\section{Supplementary Material}

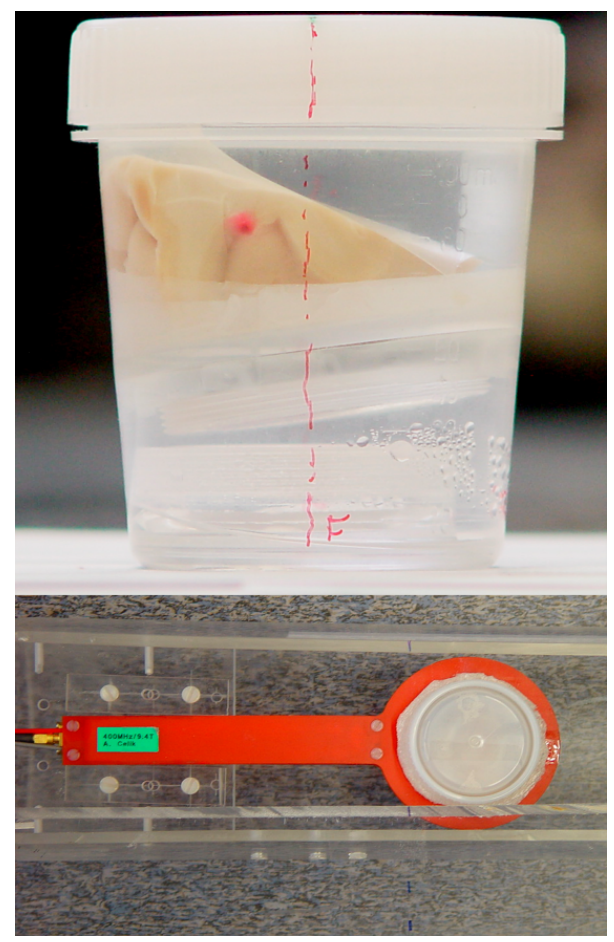

Figure S1: MR acquisition setup. Top: tissue block in its plastic container immersed in the fixation solution; a line was drawn on the container for subsequent reference for histological dissection. Bottom: container inserted in the $7 \mathrm{~cm}$ loop coil used for RF transmission and signal reception.
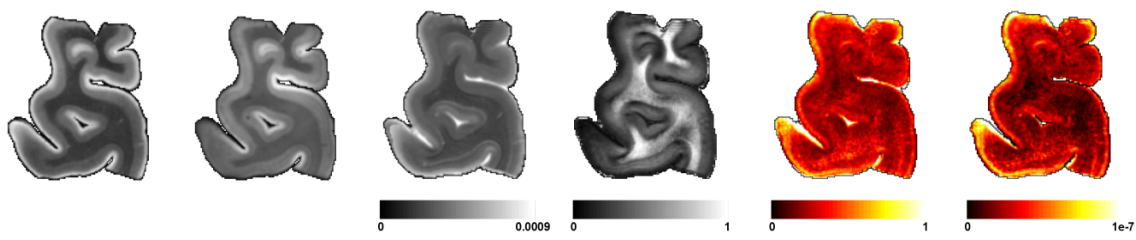

Figure S2: Basic contrast maps obtained from dMRI data contrast-optimized for gray matter differences. From left to right: mean over the 6 non diffusion weighted (i.e. B0s or T2-weighted) volumes, mean over the 60 diffusion encoded volumes, mean diffusivity (MD) in mm2s-1, fractional anisotropy (FA), sum of squared error of the diffusion tensor fitting procedure and of the 6th order spherical harmonic fit of the ADC profile. 


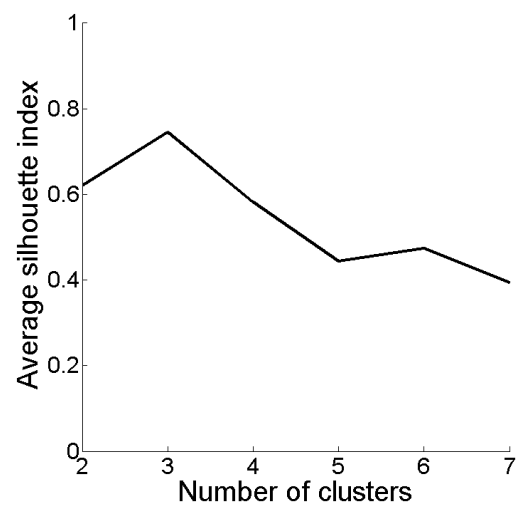

Figure S3: Silhouette analysis for the optimal number of clusters for dataset 1 showing a maximum at 3 clusters (corresponding to 2 clear layer clusters and 1 noise cluster) and another (local) maximum at 6 clusters (corresponding to 4 clear layer clusters and 2 noise clusters).

Dataset 1

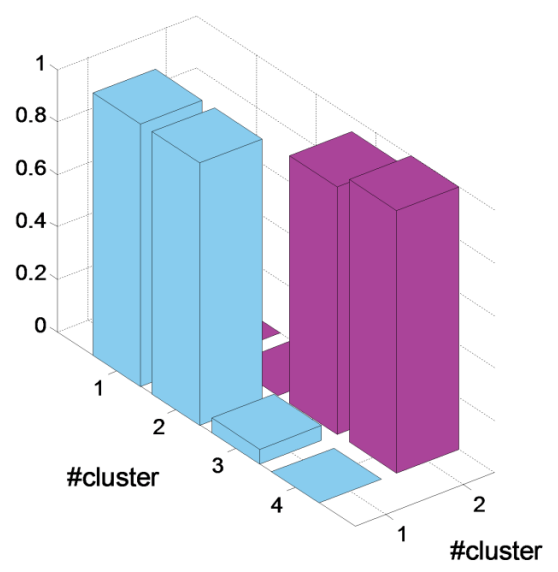

Dataset 2

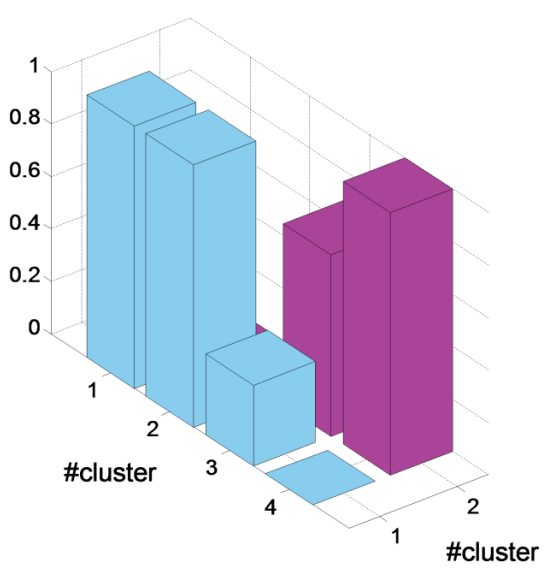

Figure S4: Crosstable correspondance analysis between the two and the four layer cluster results for dataset 1 (left) and dataset 2 (right). 

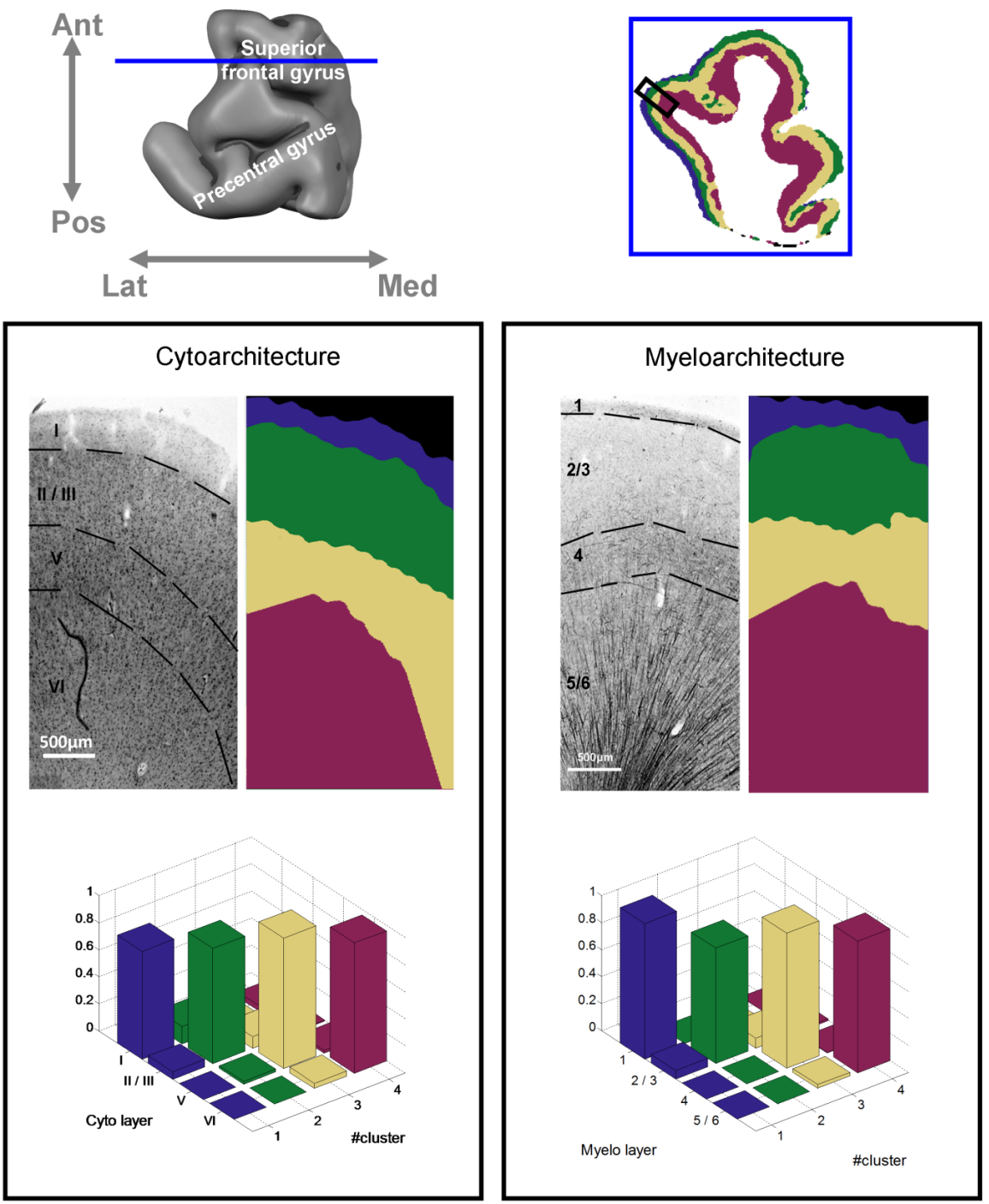

Figure S5: Correspondance between the four layer cluster result and histology on the same tissue block. Upper row, localization of the analyzed coronal section (C in Fig. 1). Lower panels, correspondance to cytoarchitecture (left) and myeloarchitecture (right). For each panel, the upper row is the histological classification of layers and dMRI layer cluster result, the bottom row is the cross-table contingency analysis between histology and dMRI-based layer clustering. In accordance with previous studies (e.g.Sanides 1962) layer IV is absent cytoarchitectonically in this cortical region. 

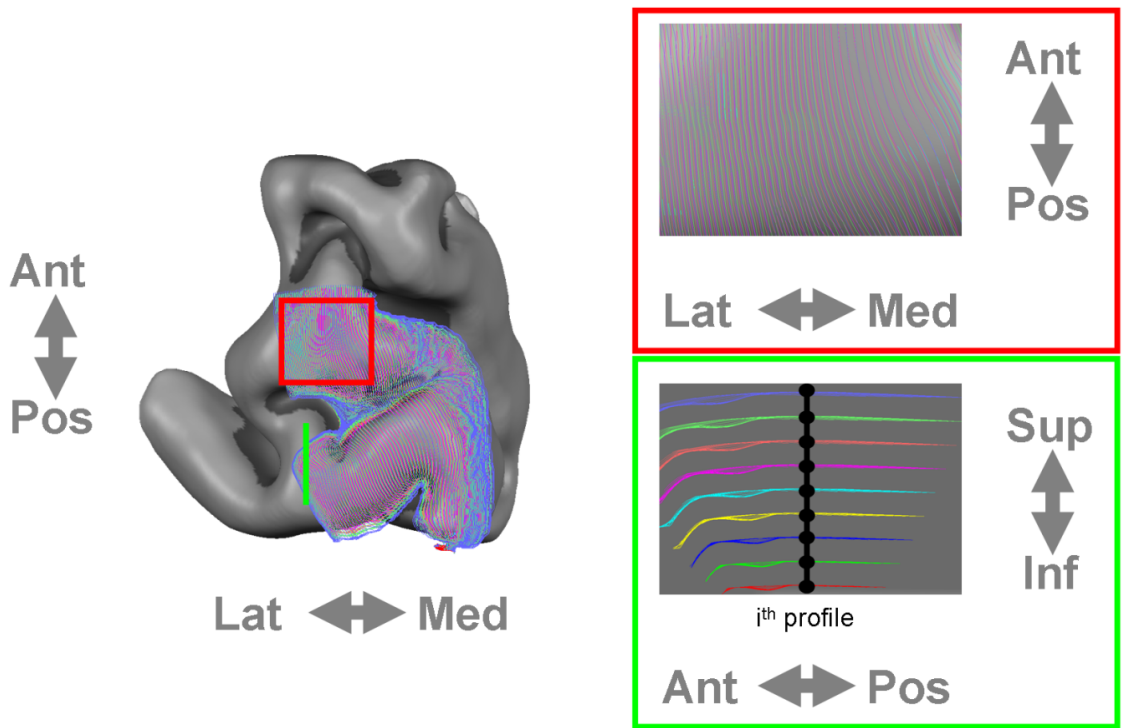

Figure S6: Three dimensional grid for area boundaries demarcation superimposed to the sampled tissue (left). Red inset: streamlines of 200 equi-spaced steps sampling along the antero-posterior direction. Green inset: sagittal view of the 9 cortical meshes sampling the gray matter volume. The ith profile is defined radially along the cortical normal at every intersection point.

\begin{tabular}{lcc}
\hline & $\begin{array}{c}\text { Cluster 1, } \\
\text { dataset 1 }\end{array}$ & $\begin{array}{c}\text { Cluster 2, } \\
\text { dataset 1 }\end{array}$ \\
\hline $\begin{array}{l}\text { Cluster 1, } \\
\text { dataset 2 }\end{array}$ & 0.94 & 0.06 \\
$\begin{array}{l}\text { Cluster 2, } \\
\text { dataset 2 }\end{array}$ & 0.01 & 0.99 \\
\hline
\end{tabular}

Table S1: Normalized crosstable for the two layer cluster analysis for dataset 1 and 2 over the whole sampled gray matter volume. Diagonal mean $=0.97$. See also Fig. 2. 


\begin{tabular}{lcccc}
\hline & $\begin{array}{c}\text { Cluster 1, } \\
\text { dataset 1 }\end{array}$ & $\begin{array}{c}\text { Cluster 1, } \\
\text { dataset 1 }\end{array}$ & $\begin{array}{c}\text { Cluster 1, } \\
\text { dataset 1 }\end{array}$ & $\begin{array}{l}\text { Cluster 1, } \\
\text { dataset 1 }\end{array}$ \\
\hline $\begin{array}{l}\text { Cluster 1, } \\
\text { dataset 2 }\end{array}$ & 0.97 & 0.03 & 0 & 0 \\
$\begin{array}{l}\text { Cluster 2, } \\
\text { dataset 2 }\end{array}$ & 0.13 & 0.86 & 0.01 & 0 \\
$\begin{array}{l}\text { Cluster 3, } \\
\text { dataset 2 }\end{array}$ & 0 & 0.21 & 0.79 & 0 \\
$\begin{array}{l}\text { Cluster 4, } \\
\text { dataset 2 }\end{array}$ & 0 & 0 & 0.19 & 0.81 \\
\hline
\end{tabular}

Table S2: Normalized crosstable for the four layer cluster analysis for dataset 1 and 2 over the whole sampled gray matter volume. Diagonal mean $=0.86$. See also Fig. 2 .

\begin{tabular}{lcccc}
\hline & Cluster 1 & Cluster 2 & Cluster 3 & Cluster 4 \\
\hline Myelo layer 1 & 0.95 & 0.02 & 0.02 & 0.01 \\
Myelo layer 2 / 3 & 0.05 & 0.95 & 0 & 0 \\
Myelo layer 4 & 0 & 0.11 & 0.87 & 0.02 \\
Myelo layer 5 / 6 & 0 & 0 & 0.04 & 0.96 \\
\hline
\end{tabular}

Table S3: Normalized crosstable for the four layer cluster analysis and myeloarchitectonics for section B, dataset 1 . Diagonal mean $=0.93$. See also Fig. 3 .

\begin{tabular}{lcccc}
\hline & Cluster 1 & Cluster 2 & Cluster 3 & Cluster 4 \\
\hline Cyto layer I & 0.94 & 0.06 & 0 & 0 \\
Cyto layer II / III & 0.04 & 0.83 & 0.13 & 0 \\
Cyto layer IV & 0 & 0.01 & 0.99 & 0 \\
Cyto layer V / VI & 0 & 0 & 0.09 & 0.91 \\
\hline
\end{tabular}

Table S4: Normalized crosstable for the four layer cluster analysis and cytoarchitectonics for section B, dataset 1 . Diagonal mean = 0.92. See also Fig. 3. 
Chapter 4 


\section{Chapter 5}

\section{Probing white matter microstructural compartments with diffusion MRI: high spatial resolution and partial volume effects}

Corresponding publication:

Bastiani, M., De Santis, S., Jones, D., Goebel, R. and Roebroeck, A. (submitted). Probing white matter microstructural compartments with diffusion MRI: high spatial resolution and partial volume effects. 


\section{Abstract}

Diffusion Tensor Imaging (DTI) has proven to be a valuable tool to investigate the microstructural properties of white matter in the brain. However, indices derived from DTI are influenced by different factors, such as axonal density, fiber configuration within a voxel or degree of myelination and thus lack specificity. Modern diffusion microstructure compartment modeling techniques describe the diffusion magnetic resonance imaging (dMRI) signal as a combination of two components: the hindered (extra-axonal) and restricted (intra-axonal) compartment. The estimation of the volume fraction of these two water pools usually requires long acquisition times. Thus studies aimed at quantifying microstructural properties of white matter have either limited resolution or accepted long measurement times. In this work, we investigate the feasibility of probing white matter microstructure using the composite hindered and restricted model of diffusion (CHARMED) model at high spatial resolution in vivo at $3 \mathrm{~T}$. We combine protocol optimization, simultaneous multi-slice/multi-band imaging and a high amplitude gradient set to enable short-time high-resolution CHARMED acquisitions. We show that short-time high-resolution CHARMED acquisitions are feasible when using the three methods combined and provide optimized reference protocols. Furthermore, we evaluate resolution and partial volume effects for the microstructure-specific restricted fraction (FR) index. We show that at higher resolution the estimated FR will tend to be higher. We investigate to which degree these findings are caused by partial volume effects between different tissue types characterized by distinct microstructural properties. We conclude that relative differences between white matter tracts are robustly detected over the range of investigated resolutions, confirming the reliability of diffusion microstructure estimation with controlled acquisition protocols. 


\section{Introduction}

Over the last two decades, diffusion magnetic resonance imaging (dMRI) has proven to be an invaluable tool to investigate the microstructural properties of white matter in the brain. Basic scalar indices obtained from fitting a diffusion tensor (DT; Basser et al., 1994) to dMRI data are sensitive to different structural properties of the brain, but lack specificity (Pierpaoli and Basser, 1996). Mean diffusivity (MD) and fractional anisotropy (FA) are influenced by different factors, such as axonal density, fiber configuration within a voxel or degree of myelination (for review, see: Alexander et al., 2007; Beaulieu, 2002; Concha, 2014). Therefore, changes in these indices cannot be attributed to a specific biological feature that characterizes the brain's microstructural organization and its change due to aging, plasticity and pathological conditions.

Recently, new modeling approaches based on in vivo dMRI data have been proposed capable of estimating quantities specific to microstructural organization of the brain. These approaches use the fact that different physiological compartments within white matter constrain the diffusion of water molecules in different ways. There are two main pools in which water diffusion is anisotropic and can be probed with dMRI: the intra-axonal and an extra-axonal compartment. Water diffusion inside the axonal membrane is restricted with a limit on diffusion distance, while diffusion within the extracellular space is be hindered. The latter can be approximated using the Gaussian model inherent to diffusion tensor imaging function, but for restricted diffusion the Gaussian assumption does not hold. These differences in diffusion behavior within the two compartments are then reflected in the acquired signal when using a PGSE sequence (Stanisz et al., 1997). The main rationale behind the several available microstructural mapping approaches is to estimate the different dMRI signal contribution arising from separate water pools in quantitative volume 
fractions (Assaf and Basser, 2005; Jespersen et al., 2007; Panagiotaki et al., 2012; Zhang et al., 2012).

The composite hindered and restricted model of diffusion (CHARMED; Assaf and Basser, 2005), in particular, models the intraaxonal restricted volume fraction (FR) using the framework developed by Neuman. This framework describes the diffusive behavior of water molecules in impermeable cylinders having a finite radius and an infinite length (Neuman, 1974). The signal from extra-axonal hindered volume fraction is described using the standard DTI model (Basser et al., 1994; Basser and Pierpaoli, 1996). Within the CHARMED model it is possible to estimate more than one intra-axonal compartment per voxel and, thus, to account for multiple fiber orientations. Several microstructural parameters can be obtained from the CHARMED analysis, such as axonal orientations, water diffusivities and anisotropies and the different water pool fractions (intra- and extraaxonal). The FR is of particular interest because it is proportional to the axonal density in a specific voxel. A recent study has shown that FR, due to its improved microstructural specificity, is much more sensitive to short term neuro-plasticity changes after a learning experiment rather than MD derived from the DT (Tavor et al., 2013).

However, with current MRI hardware, acceptable measurement times ( $<$ 10-15 min.) for even moderate spatial resolutions $(<3 \mathrm{~mm}$ isotropic) are not easily achievable, especially in clinical settings. A typical CHARMED acquisition requires data sampled at different bvalues typically up to $6000-8000 \mathrm{~s} / \mathrm{mm}^{2}$. Furthermore, at each b-value multiple diffusion encoding directions must be acquired. As a result, long acquisition times are needed to sample data over all b-values and diffusion directions. Thus, so far studies aimed at quantifying microstructural properties of white matter have either limited resolution or accepted long measurement times.

It has been shown that data resolution influences scalar indices obtained from standard DTI analysis. DTI-based FA and MD were 
found to significantly vary across two different resolutions in the study by Papanikolaou et al. (2006). They reported FA to be higher when acquiring data at higher resolution, while MD showed the opposite behavior. They further investigated their results by correlating SNR maps with FA and MD difference maps and showed that higher differences in scalar values were located in low SNR areas. Moreover, another recent study addressed the issue of partial volume effects (PVEs) when looking at DTI scalar values. In their study, Vos et al. (2011) showed that both FA and MD are affected by bundle volume, curvature and orientation. The strongest influence was given by bundle volume, which was correlated to the changes in FA and MD caused by different estimates of axial and radial diffusivity in bundles which are located close to CSF (corpus callosum and cingulum).

In this work we use a combination of three methodological innovations to enable short-time high-resolution CHARMED acquisitions. First, we use dMRI acquisition protocol optimization. Finding the optimal combination of $b$-values and diffusion encoding directions is fundamental to shorten total acquisition times without sacrificing precision and accuracy of estimated model parameters (Alexander, 2008; Jones, 2004; Prckovska et al., 2013; Zhang et al., 2012). In this work, we extend the work of De Santis et al. (2013) to reduce the total acquisition time of high spatial resolution CHARMED acquisitions of $2 \mathrm{~mm}, 1.5 \mathrm{~mm}$ and $1.3 \mathrm{~mm}$ isotropic. Second, we use simultaneous multi-slice/multi-band echo-planar imaging (SMS/MB; Feinberg et al., 2010; Moeller et al., 2010; Setsompop et al., 2012; Sotiropoulos et al., 2013a) that allows acquiring multiple slices during a single echo planar imaging (EPI) readout. Multi-band (MB) factors of 2 or 3 can therefore decrease the total scanning time to half or a third of the time required when using standard PGSE sequences. Third, as achievable signal-tonoise ratio (SNR) for dMRI increases dramatically with the available diffusion gradient amplitude, we use a clinical 3T MRI scanner equipped with a high strength $(80 \mathrm{mT} / \mathrm{m})$ gradient system. We show 
that short-time high-resolution CHARMED acquisitions are feasible when using the three methods combined and provide optimized reference protocols. Subsequently, we use acquired data on the same subjects over multiple resolutions to address the effects of spatial resolution and partial volume in the estimation of FR.

\section{Materials and Methods}

\section{Participants}

Four right-handed participants ( 2 males, 2 females, mean age $=28.25$ years, $S D=2.06$ ) took part in the experiment. All had no history of neurological or psychiatric disorders. Before the start of the experiment, written and oral informed consent was obtained and approval for this study was granted by the Ethical Committee of the Faculty of Psychology and Neuroscience at Maastricht University.

\section{Protocol Optimization}

Diffusion microstructure protocol optimization was performed extending the approach of De Santis et al. (2013) to account for different spatial resolutions and the correspondingly different SNR. The optimization procedure aims at maintaining the angular resolution of the estimated axonal directions and the accuracy of FR values that can be obtained compared to a high number of directions orientation scheme for every sampled b-value.

As a first step, 100 consecutive diffusion un-weighted (b0) scans were acquired at three different resolutions $\left(2.0,1.5\right.$ and $1.3 \mathrm{~mm}^{3}$ isotropic voxels). The time-series SNR of the b0 scan was then measured in white matter as the ratio between the voxel-wise mean and the standard deviation of the distribution of b0 intensity values over the images. The time-series SNR measured in this way is a mixture of single image (thermal) SNR, physiological noise and image stability. Since all of these factors need to be taken into account in the context of in vivo 
imaging, the time-series SNR was taken as the best reference to guide protocol optimization. Synthetic data were then generated by MonteCarlo simulations for the three different resolutions in order to identify the best protocol in terms of total scanning time and accuracy of estimated FR for each dataset. Monte-Carlo simulations were performed using the CAMINO toolkit (Hall and Alexander, 2009) with the following parameters: 50000 walkers, 20000 time steps, restricted volume fraction of 0.42 , one and two orthogonal fiber populations with a radii distribution drawn from a gamma distribution of parameters $\mathrm{a}=$ 9.1477 and $b=4.977 \mathrm{~mm}$ (as in: Aboitiz et al., 1992), longitudinal diffusivity equal to $10^{-3} \mathrm{~mm}^{2} / \mathrm{s}$. For each acquisition scheme, the shortest $\Delta$ and the longest $\delta$ combination that matches the desired b-value and that minimizes TE was chosen.

Furthermore, for each spatial resolution, simulated data were generated for 8 different maximum $b$-values, ranging from $b$-max $=1000$ $\mathrm{s} / \mathrm{mm}^{2}$ to $10000 \mathrm{~s} / \mathrm{mm}^{2}$. For each b-max, a protocol consisted of 8 equally spaced b-values where 200 gradient orientations were generated on a sphere for each b-value. The simulations were repeated 30 times for each scheme (consisting of a single b-max) using different random starting conditions. Simulated annealing was used to optimize the order in which the gradient orientations were sampled. In this way, the sampling of the dataset is uniform even when only a fraction of the gradient orientations can be acquired because of hardware failure or subject's compliance (Cook et al., 2007; Dubois et al., 2006). To identify the shortest protocol, we truncated the ordered datasets by removing one measurement at a time from the end of the acquisition, and ran the CHARMED analysis on the reduced dataset.

The mean of the CHARMED parameters was used to evaluate the performance of each of the schemes. Specifically, the accuracy was calculated as the difference between the nominal and the measured mean values. The best scheme was then established by finding, amongst all the combinations, the shortest possible protocol that maintains 
accuracy for FR and fiber orientation below a bias of 5\%. The resulting high resolution diffusion microstructure protocols are listed in Appendix A.

For in-vivo data acquisitions with the optimized protocol, TE, bvalue and diffusion gradient parameters $(\Delta / \delta)$ were kept equal in order to make controlled comparisons between different spatial resolutions. Therefore, the b-value that allowed the highest accuracy with the lowest number of measurements for all three resolutions together was chosen. This was $\mathrm{b}=6000 \mathrm{~s} / \mathrm{mm}^{2}$, which was therefore chosen for performing the in-vivo data acquisitions. Lastly, looking separately at the simulation results for the different resolutions we identified the number of gradient orientations needed for each protocol to keep accuracy within 95\% of the gold standard. As expected, the highest resolution has the lowest SNR and this is compensated by more acquired volumes (a denser orientation sampling scheme) to match the uncertainty in the estimated FR.

\section{MR Data Acquisition}

A Siemens 3T Prisma ${ }^{\mathrm{FIT}}$ MR scanner equipped with a high amplitude gradient-coil (maximum amplitude $80 \mathrm{mT} / \mathrm{m}$, maximum slew rate 200 $\mathrm{T} / \mathrm{m} / \mathrm{s}$ ) and a 32-channel head RF-coil were used to acquire the data. The subject's head was immobilized using foam cushions. A singly refocused spin-echo diffusion multiband sequence (Sotiropoulos et al., 2013a; Sotiropoulos et al., 2013b) was used to acquire diffusion weighted data at three different resolutions (MB factor $=2$, resolution: 2.0, 1.5 and $1.3 \mathrm{~mm}^{3}$ isotropic voxels, 60-83-90 axial slices, GRAPPA 2, Partial Fourier 6/8, 6 b0s, 6 b-values), for a total of three different datasets (Table 1). The maximum b-value was set to $6000 \mathrm{~s} / \mathrm{mm}^{2}$ and TE $=94 \mathrm{~ms}$ for all acquisitions (see section "protocol optimization" for details). These two parameters were kept constant across all the acquisitions to avoid unwanted T2 weighting effects. Moreover, readout bandwidth and echo spacing were also kept similar to keep SNR image artefacts and $\mathrm{T}^{*}$ weighting stable across the different datasets. Diffusion 
gradient amplitude $(|G|)$ and pulse length $(\delta)$ were allowed to change across datasets. The described optimized protocols were used in which higher angular resolution at higher spatial resolutions compensates for lower SNR in higher resolution scans. This resulted in a total number of 45-72-108 diffusion encoding directions for the $1 \mathrm{~mm}, 1.5 \mathrm{~mm}$ and $1.3 \mathrm{~mm}$ datasets, respectively. Five further matched b0 scans with reversed phase encoding $(\mathrm{P}->\mathrm{A})$ direction were acquired after each series for correction of susceptibility induced distortions. A 3D MPRAGE acquisition $\left(1 \mathrm{~mm}^{3}\right.$ isotropic voxels, $\mathrm{TR}=2300 \mathrm{~ms}$, TE $\left.=2.98 \mathrm{~ms}\right)$ was acquired for white/grey matter segmentation and coregistration.

\section{MR Data Processing}

Diffusion data were preprocessed in order to correct for bulk motion, eddy current induced distortions and susceptibility artifacts using the phase-encode direction reversed volumes (Andersson et al., 2003) as implemented in FSL 5.0.6 (Smith et al., 2004). All further modeling and analysis was performed in 'native' diffusion data space with only final result maps being spatially transformed for alignment and comparison.

The dMRI signal was fitted to the CHARMED model using the routines described in De Santis et al. (2013) coded in MATLAB (The Mathworks, Natick, MA) and based on the Levenberg-Marquardt algorithm. FR maps were estimated for each dataset. For comparison and cross-subject combination, the FR maps were normalized to MNI space (1 $\mathrm{mm}^{3}$ isotropic voxel) using a two-step procedure as implemented in the FLIRT toolbox (Jenkinson et al., 2002; Jenkinson and Smith, 2001). First, each FR map obtained from the three acquisition protocols were coregistered to the T1 anatomical acquisition of the same subject using a rigid body transformation (6 degrees of freedom) based on the normalized mutual information cost function and trilinear interpolation. Second, each T1 volume was coregistered to the MNI template using an affine transformation (12 degrees of freedom) procedure based on the correlation ratio cost function and trilinear interpolation. The FR maps were then coregistered to the MNI template 
in one step combining the two transformation matrices. The T1 volumes were segmented using SPM 8 (Friston et al., 1994) to obtain three tissue probabilistic volumes representing white and gray matter and cerebrospinal fluid. The white matter masks were thresholded at a value of 0.98 to remove voxels at the boundaries between white and gray matter and CSF.

A tract based spatial statistics (TBSS; Smith et al., 2006) analysis was performed on a set of white matter tracts of interest. The common white matter skeleton was obtained by averaging the FR maps of the four subjects separately for every acquired resolution protocol. The white matter skeleton was therefore reconstructed at $1 \mathrm{~mm}$ resolution in the standard MNI template. We used a white matter atlas to define tracts of interest within the skeleton for the current study (Mori et al., 2008). The atlas was projected onto the skeletonized white matter maps and 48 white matter bundles were selected for all the four subjects. The average FR value for every bundle was computed and reported.

To evaluate the influence of PVE on the estimated FR values from the center to the periphery of a white matter tract, the skeletonized corpus callosum was considered. A curve was defined connecting the center of all the voxels lying in the skeletonized corpus callosum within a single mid-sagittal slice. Then, all the normals to the curve lying within the same mid-sagittal slice were computed at each of the 112 sampled points. FR values were sampled at $0.5 \mathrm{~mm}$ steps across the positive and negative directions (roughly along the inferior-superior axis in the midbody) defined by the estimated normal up to $3 \mathrm{~mm}$ away from the skeleton. The effects of PVE were evaluated focusing on the mid-body of the corpus callosum by selecting 10 consecutive sampled points (from 40 to 49). We averaged the $10 \mathrm{FR}$ profiles along the anterior-posterior direction for analysis. To minimize effects of misalignments between the center of the tract and the skeleton, we matched these $10 \mathrm{FR}$ profiles according to their maximum value along the sampled points in the normal (inferior-superior) direction. The 
effects of PVE were then evaluated by assessing the change in FR from extreme periphery to center of the CC:

$$
\text { Change }=\frac{\left(\left(F R_{-3 m m}-F R_{\max }\right)+\left(F R_{+3 m m}-F R_{\max }\right)\right)}{2 \cdot F R_{\max }}
$$

where FR-3mm and FR+3mm are the FR values sampled at $3 \mathrm{~mm}$ from the maximum of the tract following the negative and positive direction defined by the local normal respectively and FR $\max$ is the maximum FR value.

\section{Results}

Figure 1 shows a transverse slice through the FR maps for the four subjects across the three different resolutions. FR could be estimated stably with relatively low visible variation or noise within the maps over all resolutions. Large white matter tracts such as the corpus callosum and the cortiscospinal tract appear to keep the same relative values of FR between the different acquisitions. Furthermore, when going from the lowest (2 $\mathrm{mm}$ isotropic voxels) to the highest resolution $(1.3 \mathrm{~mm}$ isotropic voxels) an increase in sharpness of the boundaries between gray and white matter and CSF can be observed. More surprisingly, a slight tendency can be observed (for instance in the corpus callosum) for the FR to increase at higher spatial resolutions.

To quantitatively assess the differences and similarities between the different resolutions, Table 2 shows the mean and the standard deviation of the difference in FR in the entire white matter volume as segmented from the aligned T1 image. All the difference maps are computed by subtracting a higher acquired resolution FR map from the FR map of the same subject acquired at a lower resolution. For all the difference maps, the mean difference is always lower than zero, implying a higher estimated FR for higher resolution scans. The mean 
values are always less than 0.05 away from 0 and the standard deviations of the differences are generally more than double the respective averages. The observed FR increases range up to $10 \%$ relative to the volume with the lower resolution. This shows that there is a small but consistent and stable increase of FR values, up to about $10 \%$ of the mean WM FR values, when increasing spatial resolution almost 4 -fold (2 $\mathrm{mm}$ to $1.3 \mathrm{~mm}$ isotropic).

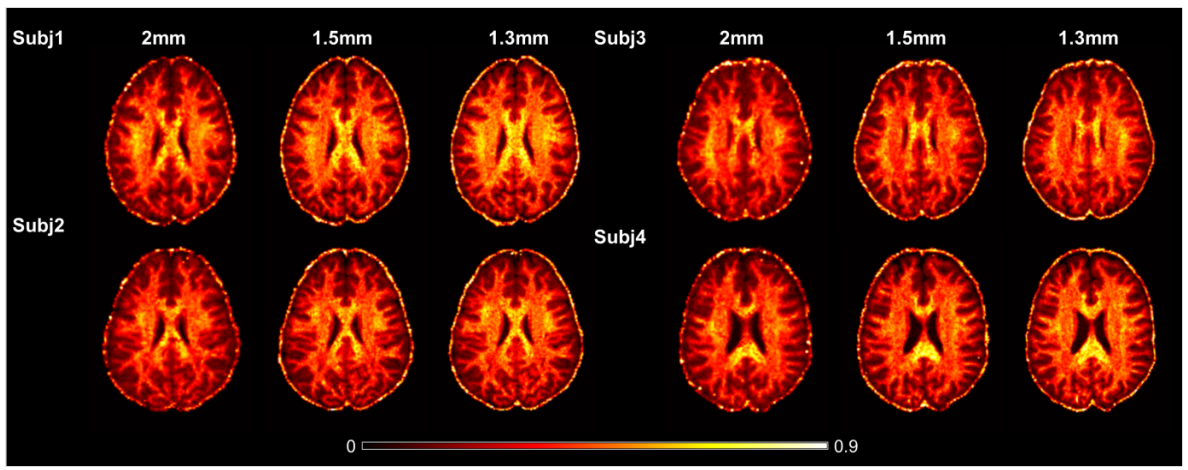

Figure 1: FR pattern of the different subjects obtained with different resolutions. Each FR map was coregistered to the standard 1mm MNI 152 template for comparability.

Figure 2 shows a more detailed analysis of the values in the FR maps in terms of $\mathrm{Q}-\mathrm{Q}$ plots and scatterplots. FR distributions for the three different resolutions were obtained by sampling the FR maps averaged across subjects along the white matter skeleton, representing tract centers, in MNI space at $1 \mathrm{~mm}$ isotropic resolution. Values obtained from the 1.3 and $1.5 \mathrm{~mm}$ isotropic volumes are very consistent as shown by the Q-Q plot and by the scatter plot analysis with its regression line close to the identity line. The FR value correlation between $1.5 \mathrm{~mm}$ and $1.3 \mathrm{~mm}$ resolution is 0.87 . Larger differences are found between the two most extreme voxel sizes tested (1.3 and 2.0 $\mathrm{mm})$. 
Q-Q plot
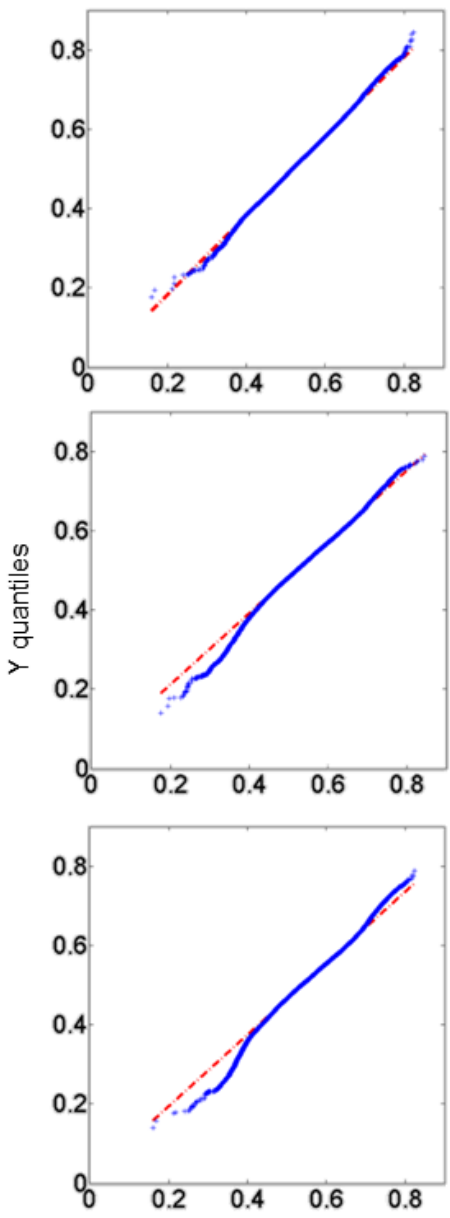

$\mathrm{X}$ quantiles
Scatter plot

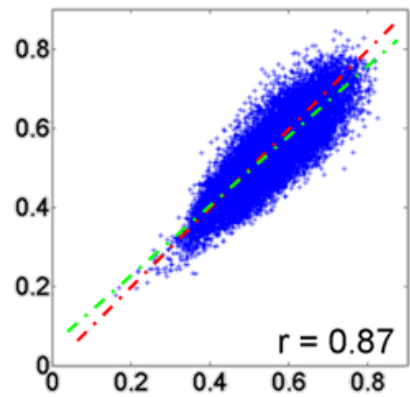

$X: 1.3 \mathrm{~mm}$

$\mathrm{Y}: 1.5 \mathrm{~mm}$

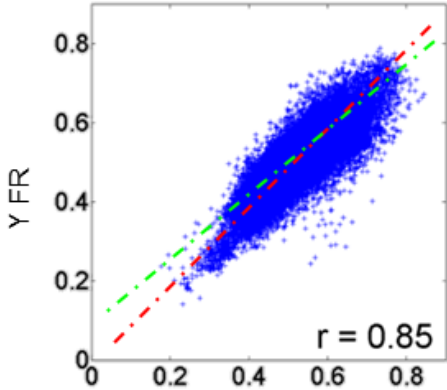

$\mathrm{X}: 1.5 \mathrm{~mm}$

$\mathrm{Y}: \mathbf{2 . 0 \mathrm { mm }}$

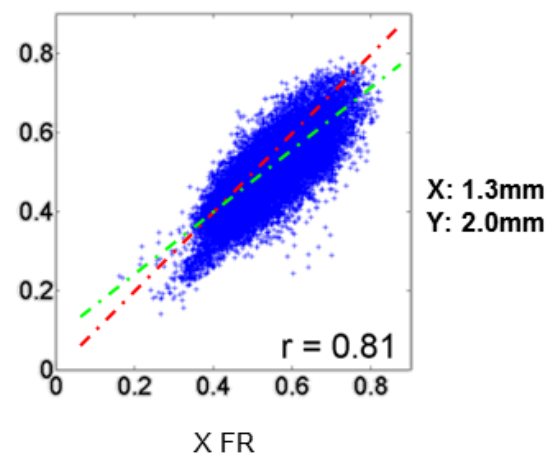

Figure 2: Q-Q plots (left column) and scatter plots (right column) comparing the FR distributions between resolutions sampled over the WM skeleton. Red line is the identity $(y=x)$ line, the green line in the scatter plot is a least square linear regression fit.

The Q-Q plot shows variations in both in the lower (0.2-0.4) and higher (0.6-0.8) quantile range and the correlation lowers slightly to 0.81 . In the scatter plots the slope of the regression line is lower than the identity line. This corresponds to higher FR values at higher resolution in the high values range, but lower FR values at higher resolution in the low 
values range, in agreement with the $\mathrm{Q}-\mathrm{Q}$ plot. However, it is clear that a majority of the points in the white matter skeleton lies in the medium to high value range, such that the high value range difference dominates. Overall, these results show that FR values over a large range (0.2-0.8) experience a "compression" of values towards the general average value at lower resolutions. Correspondingly, high FR values, as they mostly occur in white matter, are reduced at lower resolutions. These differences are smaller when operating in an already high resolution regime (e.g. $1.3-1.5 \mathrm{~mm}$ ).

Figure 3 shows the results of the TBSS analysis for the 48 tracts segmented from the white matter atlas. The average FR value for every segmented track is shown as obtained from the skeletonized white matter mask and thus reflects FR values in the center of the tract rather than the whole tract. Distinct white matter bundles are characterized by different average FR values, likely reflecting their microstructural properties. These relative differences between tracts remain stable over resolutions. However, the overall FR pattern shows the tendency of FR values to uniformly increase at higher resolutions.

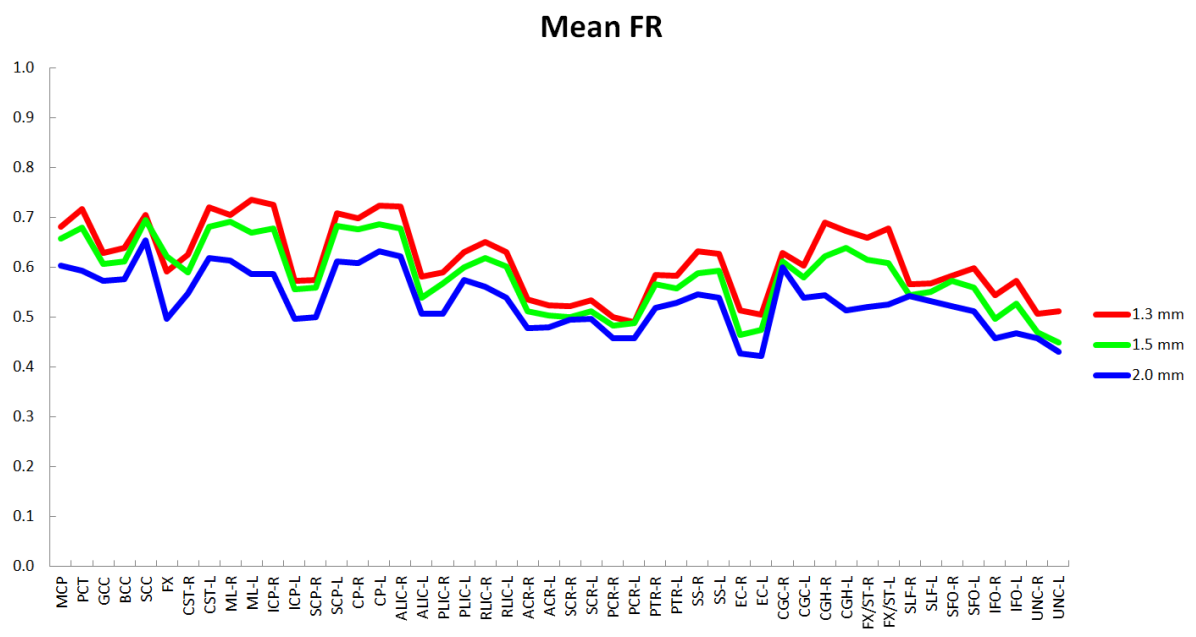

Figure 3: Mean FR plot for the 48 selected WM tracts. 

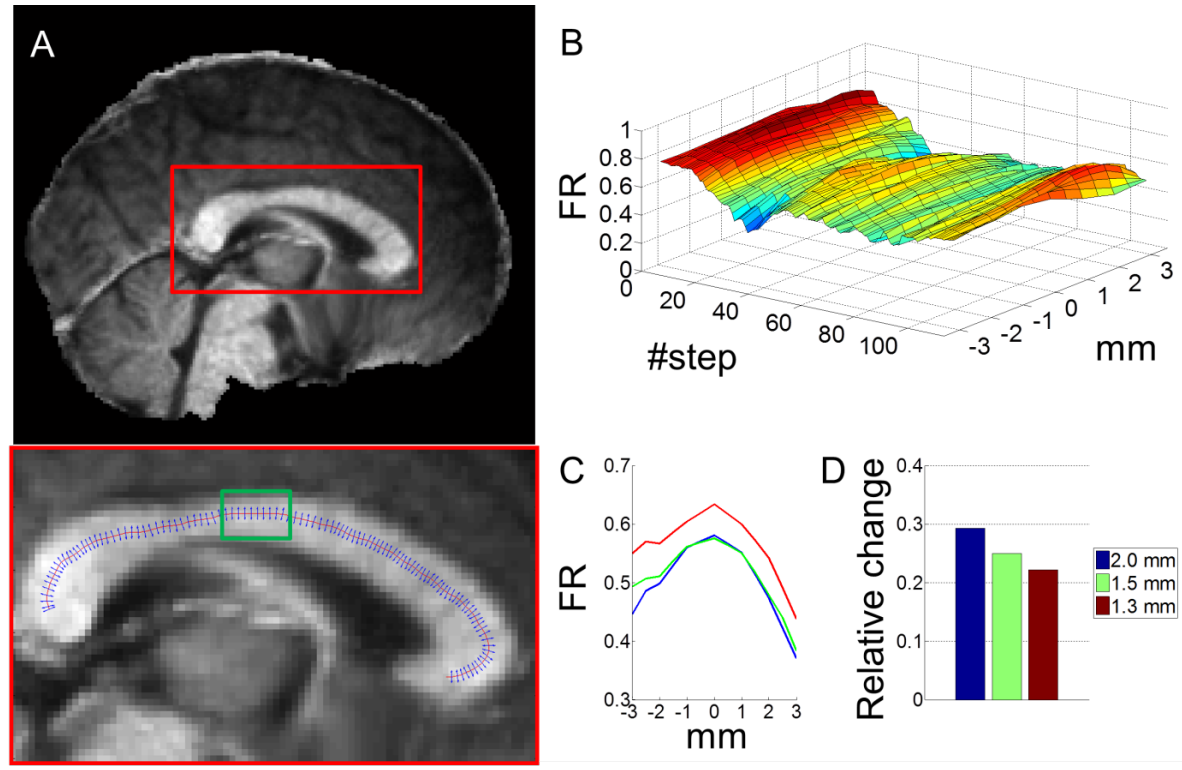

Figure 4: Analysis of partial volume effects in the corpus callosum. A) Top: Mid-sagittal slice of the averaged FR volume in $1 \mathrm{~mm}$ MNI 152 standard space. Bottom: the white matter skeleton (in red) defined at the center of the corpus callosum, the normals $(1 \mathrm{~mm}$ length in blue) to the skeleton which lie in the same mid-sagittal plane and in green the region of interest for C and D. B) FR values plotted as a function of steps along the corpus callosum skeleton, starting from the splenium to the genu of the corpus callosum, and distance from the skeleton along the normal in millimeters. C) FR values along the normal direction averaged across 10 steps (from 40 to 50, green rectangle in A lying in the mid-body of the corpus callosum. D) Relative change of FR from periphery to center of the $\mathrm{CC}$ for the acquired resolutions.

Figure 4 evaluates PVEs on the estimated FR indices when moving from the center of the $\mathrm{CC}$ to the periphery where partial voluming with CSF, large sinuses or blood vessels and gray matter tissue occurs. The surface plot in figure $4 \mathrm{~B}$ shows that the maximum FR value mostly lies within the point sampled by the white matter skeleton (the 0 point). The FR sampled along the $\mathrm{CC}$ at this point reflects the known pattern of high-low-high fiber density within the genu, body and splenium of CC, respectively (Aboitiz et al., 1992). Figure 4C again illustrates the center-of-tract difference within the corpus callosum, showing differences in FR at the 0 point. Furthermore, it shows that the 
FR decreases with distance away from the maximum in the center of the tract as expected with increasing PVE. Figure D shows that FR changes more towards the tract periphery at lower resolutions with a relative change of 0.28 for the $2.0 \mathrm{~mm}$ isotropic volume.

\section{Discussion}

We have created a set of optimized protocols to probe white matter microstructure in vivo beyond the DTI model at high spatial resolution using a 3T MRI scanner. Using simultaneous multi-slice/multi-band imaging and a high amplitude gradient set, we show that a maximum resolution of $1.3 \mathrm{~mm}$ isotropic voxels is achievable within a total scan time of less than 15 minutes. This could possibly be even further reduced by using less conservative SMS/MB factors, e.g. a 10 minute acquisition would be possible with SMS/MB 3. Furthermore, we investigated the variation of intra-axonal volume fraction (FR) with resolutions and assessed PVEs.

It has recently been shown that a clinically feasible scanning time was reachable for CHARMED acquisitions using optimized protocols at moderate spatial resolution (De Santis et al., 2013). The optimization in that study was focused on q-space sampling optimization and considered standard clinical settings, with hardware and pulse sequences which are commonly available in the clinic. The study suggested a protocol with $3 \mathrm{~mm}$ isotropic resolution in a total acquisition time of 12 minutes. With recent hardware and acquisition developments, in particularly higher maximum gradient strength and SMS/MB PGSE EPI acquisition, data can be acquired in a more efficient way. In this work, we have shown that with an optimal combination of diffusion and image space sampling, both high resolution and short total acquisition times are feasible for dMRI microstructure compartment modeling studies. A $2 \mathrm{~mm}$ isotropic acquisition could be performed in less than 5 minutes. Taking as a reference the $3 \mathrm{~mm}$ 
isotropic acquisition proposed in the study by De Santis el al. (2013), our SMS/MB $2 \mathrm{~mm}$ isotropic acquisition protocol represents a $\sim 3$-fold resolution increase with a simultaneous $\sim 2$-4-fold acquisition time speedup. With the setup we used in the current work, we show that a $1.3 \mathrm{~mm}$ isotropic resolution (a $\sim 12$-fold resolution increase) can be acquired in a scanning time which is comparable to the previously supported $3 \mathrm{~mm}$ protocol.

We show that contrast patterns in FR maps are highly consistent across the three different resolution acquired for in vivo subjects. That is, the overall pattern of FR is very stable across the different datasets and FR shows a very high contrast-to-noise ratio (CNR) when looking at the differences between white matter voxels from gray matter ones (Fig. 1). Furthermore, when increasing the resolution it can be observed that better delineation of finer structural details can be achieved. The boundary between white and gray matter become sharper because of decreased PVE. Moreover, an atlas-based analysis of tract centers (Figure 3) shows that tract specific microstructure index FR maintains it distinctive contrast between tracts. We assessed the variation of the estimated FRs with increasing resolution by computing the difference maps across resolutions (Table 2). All the distributions are very close, but not equal, to 0 . We show that, at a whole brain white matter level, the average lower-higher resolution difference is always negative, i.e. at a higher resolution the estimated FR will tend to be slightly higher.

To address this finding, we performed three different analyses: we first focused on the quantitative similarity between the three different acquisitions by considering only the middle of the tracts using the white matter skeleton (Figure 2). Here we showed that FR values are highly correlated at the whole brain level and that, when differences are found, they tend to be stronger between extremely different resolutions, with higher FR values at higher resolution in the high-value range, but lower FR values at higher resolution in the low-value range. We extended the analysis by considering a set of 48 tracts of interest. We 
show that FR has the tendency to increase when the resolution is higher but that tract-specific average values are very correlated and consistent between each other. More specifically, looking at the genu (GCC), the body (BCC) and the splenium (SCC) of the corpus callosum, we see that the known axonal density trend, with the highest density being in the splenium (Aboitiz et al., 1992), is respected for all the different acquisitions. Lastly, to investigate to which degree these findings are caused by PVE between different tissue types characterized by distinct microstructural properties, we focused on the mid-body of the corpus callosum (Fig. 4).

It has been shown that differences in voxel size significantly influence the estimate of DTI-based FA and MD. In the study by Papanikolaou et al. (2006), FA increases when the resolution is higher, while MD shows the opposite behavior which is explained by differences in image SNR between the acquisition protocols. In our work we took care to compensate the decrease in image SNR by increasing the number of sampled diffusion orientations. Furthermore, another recent study showed that both FA and MD are affected by bundle volume, curvature and orientation because on their influence on PVE (Vos et al., 2011). Here we show that FR increases with higher resolution at the center of the white matter tracts, where FR values tend to be larger, and that it decreases when moving away from it. The higher hindered compartment fraction which can be observed in CSF or gray matter can explain the decrease in FR when moving away from the center of the tract as defined by the white matter skeleton. Furthermore, higher FR can be a result of pure image SNR differences as postulated by Papanikolaou et al. (2006). Another reason might be that, since FR is proportional to axonal density, it is also influenced by the restricted compartments of adjacent structures which lie within the same voxel and that have different microstructural characteristics. This effect can also be observed in Fig. 3, where a compression of FR values in the 0.20.8 range can be explained by the tendency to regress to the general 
average value at lower resolutions. The increase in spatial specificity obtained when acquiring data at higher resolution could then be the reason for the observed increase in FR. These effects seem to reach a plateau at the resolution of $1.5 \mathrm{~mm}$ isotropic, with only minor FR differences with the volume acquired at $1.3 \mathrm{~mm}$ isotropic, but this finding will need further investigation.

Voxel resolutions of $1 \mathrm{~mm}$ and below at high b-values (> 3000 $\mathrm{s} / \mathrm{mm}^{2}$ ) can currently only be reached for post mortem specimens using pre-clinical scanners equipped with very high amplitude gradients ( $>$ $600 \mathrm{mT} / \mathrm{m})$. We show that with modern hardware and protocol optimization a resolution of $1.3 \mathrm{~mm}$ can be achieved for in-vivo scanning sessions targeted to investigate the microstructural properties of white matter. Furthermore, such an increase in resolution opens the possibility to start investigating the structural architecture of cortical gray matter. On average, cortical thickness ranges between 2 and $4 \mathrm{~mm}$. Therefore, up to three voxels can now be sampled along the cortical normal which can be analyzed using microstructural models. This has important implication for future anatomical studies which try to address the layered structure and parcellation of cortical gray matter from a microstrucutral point of view, given that every layer is characterized by a different neurite densities and orientations. 


\section{References}

Aboitiz, F., Scheibel, A.B., Fisher, R.S., Zaidel, E., 1992. Fiber composition of the human corpus callosum. Brain Res 598, 143-153.

Alexander, A.L., Lee, J.E., Lazar, M., Field, A.S., 2007. Diffusion tensor imaging of the brain. Neurotherapeutics 4, 316-329.

Alexander, D.C., 2008. A general framework for experiment design in diffusion MRI and its application in measuring direct tissue-microstructure features. Magn Reson Med 60, 439-448.

Andersson, J.L., Skare, S., Ashburner, J., 2003. How to correct susceptibility distortions in spin-echo echo-planar images: application to diffusion tensor imaging. Neuroimage 20, 870-888.

Assaf, Y., Basser, P.J., 2005. Composite hindered and restricted model of diffusion (CHARMED) MR imaging of the human brain. Neuroimage 27, 48-58.

Basser, P.J., Mattiello, J., LeBihan, D., 1994. MR diffusion tensor spectroscopy and imaging. Biophys J 66, 259-267.

Basser, P.J., Pierpaoli, C., 1996. Microstructural and physiological features of tissues elucidated by quantitative-diffusion-tensor MRI. J Magn Reson B 111, 209-219.

Beaulieu, C., 2002. The basis of anisotropic water diffusion in the nervous system - a technical review. Nmr in Biomedicine 15, 435-455.

Concha, L., 2014. A macroscopic view of microstructure: Using diffusion-weighted images to infer damage, repair, and plasticity of white matter. Neuroscience 276C, 14-28.

Cook, P.A., Symms, M., Boulby, P.A., Alexander, D.C., 2007. Optimal acquisition orders of diffusion-weighted MRI measurements. J Magn Reson Imaging 25, 1051-1058.

De Santis, S., Assaf, Y., Evans, C.J., Jones, D.K., 2013. Improved precision in CHARMED assessment of white matter through sampling scheme optimization and model parsimony testing. Magn Reson Med.

Dubois, J., Poupon, C., Lethimonnier, F., Le Bihan, D., 2006. Optimized diffusion gradient orientation schemes for corrupted clinical DTI data sets. MAGMA 19, 134-143.

Feinberg, D.A., Moeller, S., Smith, S.M., Auerbach, E., Ramanna, S., Gunther, M., Glasser, M.F., Miller, K.L., Ugurbil, K., Yacoub, E., 2010. Multiplexed echo planar imaging for sub-second whole brain FMRI and fast diffusion imaging. PLoS One 5, e15710.

Friston, K.J., Holmes, A.P., Worsley, K.J., Poline, J.P., Frith, C.D., Frackowiak, R.S.J., 1994. Statistical parametric maps in functional imaging: A general linear approach. Human Brain Mapping 2, 189-210.

Hall, M.G., Alexander, D.C., 2009. Convergence and parameter choice for Monte-Carlo simulations of diffusion MRI. IEEE Trans Med Imaging 28, 1354-1364. 
Jenkinson, M., Bannister, P., Brady, M., Smith, S., 2002. Improved optimization for the robust and accurate linear registration and motion correction of brain images. Neuroimage 17, 825-841.

Jenkinson, M., Smith, S., 2001. A global optimisation method for robust affine registration of brain images. Medical Image Analysis 5, 143-156.

Jespersen, S.N., Kroenke, C.D., Ostergaard, L., Ackerman, J.J., Yablonskiy, D.A., 2007. Modeling dendrite density from magnetic resonance diffusion measurements. Neuroimage 34, 1473-1486.

Jones, D.K., 2004. The effect of gradient sampling schemes on measures derived from diffusion tensor MRI: A Monte Carlo study. Magnetic Resonance in Medicine 51, 807815.

Moeller, S., Yacoub, E., Olman, C.A., Auerbach, E., Strupp, J., Harel, N., Ugurbil, K., 2010. Multiband multislice GE-EPI at 7 tesla, with 16-fold acceleration using partial parallel imaging with application to high spatial and temporal whole-brain fMRI. Magn Reson Med 63, 1144-1153.

Mori, S., Oishi, K., Jiang, H., Jiang, L., Li, X., Akhter, K., Hua, K., Faria, A.V., Mahmood, A., Woods, R., Toga, A.W., Pike, G.B., Neto, P.R., Evans, A., Zhang, J., Huang, H., Miller, M.I., van Zijl, P., Mazziotta, J., 2008. Stereotaxic white matter atlas based on diffusion tensor imaging in an ICBM template. Neuroimage 40, 570-582.

Neuman, C.H., 1974. Spin echo of spins diffusing in a bounded medium. The Journal of Chemical Physics 60, 4508-4511.

Panagiotaki, E., Schneider, T., Siow, B., Hall, M.G., Lythgoe, M.F., Alexander, D.C., 2012. Compartment models of the diffusion MR signal in brain white matter: a taxonomy and comparison. Neuroimage 59, 2241-2254.

Papanikolaou, N., Karampekios, S., Papadaki, E., Malamas, M., Maris, T., Gourtsoyiannis, N., 2006. Fractional anisotropy and mean diffusivity measurements on normal human brain: comparison between low-and high-resolution diffusion tensor imaging sequences. Eur Radiol 16, 187-192.

Pierpaoli, C., Basser, P.J., 1996. Toward a quantitative assessment of diffusion anisotropy. Magn Reson Med 36, 893-906.

Prckovska, V., Achterberg, H.C., Bastiani, M., Pullens, P., Balmashnova, E., Ter Haar Romeny, B.M., Vilanova, A., Roebroeck, A., 2013. Optimal short-time acquisition schemes in high angular resolution diffusion-weighted imaging. Int J Biomed Imaging 2013, 658583 .

Setsompop, K., Cohen-Adad, J., Gagoski, B.A., Raij, T., Yendiki, A., Keil, B., Wedeen, V.J., Wald, L.L., 2012. Improving diffusion MRI using simultaneous multi-slice echo planar imaging. Neuroimage 63, 569-580.

Smith, S.M., Jenkinson, M., Johansen-Berg, H., Rueckert, D., Nichols, T.E., Mackay, C.E., Watkins, K.E., Ciccarelli, O., Cader, M.Z., Matthews, P.M., Behrens, T.E., 2006. Tract- 
based spatial statistics: voxelwise analysis of multi-subject diffusion data. Neuroimage 31, 1487-1505.

Smith, S.M., Jenkinson, M., Woolrich, M.W., Beckmann, C.F., Behrens, T.E., JohansenBerg, H., Bannister, P.R., De Luca, M., Drobnjak, I., Flitney, D.E., Niazy, R.K., Saunders, J., Vickers, J., Zhang, Y., De Stefano, N., Brady, J.M., Matthews, P.M., 2004. Advances in functional and structural MR image analysis and implementation as FSL. Neuroimage 23 Suppl 1, S208-219.

Sotiropoulos, S.N., Jbabdi, S., Xu, J., Andersson, J.L., Moeller, S., Auerbach, E.J., Glasser, M.F., Hernandez, M., Sapiro, G., Jenkinson, M., Feinberg, D.A., Yacoub, E., Lenglet, C., Van Essen, D.C., Ugurbil, K., Behrens, T.E., Consortium, W.U.-M.H., 2013a. Advances in diffusion MRI acquisition and processing in the Human Connectome Project. Neuroimage 80, 125-143.

Sotiropoulos, S.N., Moeller, S., Jbabdi, S., Xu, J., Andersson, J.L., Auerbach, E.J., Yacoub, E., Feinberg, D., Setsompop, K., Wald, L.L., Behrens, T.E.J., Ugurbil, K., Lenglet, C., 2013b. Effects of Image Reconstruction on Fiber Orientation Mapping From Multichannel Diffusion MRI: Reducing the Noise Floor Using SENSE. Magnetic Resonance in Medicine 70, 1682-1689.

Stanisz, G.J., Szafer, A., Wright, G.A., Henkelman, R.M., 1997. An analytical model of restricted diffusion in bovine optic nerve. Magn Reson Med 37, 103-111.

Tavor, I., Hofstetter, S., Assaf, Y., 2013. Micro-structural assessment of short term plasticity dynamics. Neuroimage 81, 1-7.

Vos, S.B., Jones, D.K., Viergever, M.A., Leemans, A., 2011. Partial volume effect as a hidden covariate in DTI analyses. Neuroimage 55, 1566-1576.

Zhang, H., Schneider, T., Wheeler-Kingshott, C.A., Alexander, D.C., 2012. NODDI: practical in vivo neurite orientation dispersion and density imaging of the human brain. Neuroimage 61, 1000-1016. 


\section{Appendix A}

Optimized CHARMED Gradient Orientations

For all tables direction vector length scales gradient amplitude and determines $b$-value. Each table contains measurements at 8 distinct $b$ values.

45 diffusion encoding directions for CHARMED at $2.0 \mathrm{~mm}$ iso

\begin{tabular}{ccc}
\hline $\mathbf{X}$ & $\mathbf{Y}$ & $\mathbf{Z}$ \\
\hline-0.054 & 0.229 & -0.264 \\
0.146 & -0.476 & 0.049 \\
-0.497 & 0.235 & 0.27 \\
0.043 & -0.257 & 0.657 \\
-0.368 & -0.252 & 0.653 \\
-0.832 & -0.069 & 0.229 \\
-0.337 & 0.838 & 0.243 \\
0.318 & 0.877 & 0.36 \\
0.216 & -0.271 & -0.067 \\
-0.062 & -0.069 & -0.491 \\
-0.015 & 0.137 & -0.597 \\
-0.018 & -0.428 & -0.563 \\
0.764 & -0.185 & -0.089 \\
0.578 & -0.026 & 0.644 \\
-0.098 & -0.808 & 0.46 \\
-0.542 & -0.268 & -0.797 \\
-0.272 & -0.063 & 0.217 \\
-0.474 & -0.138 & 0.079 \\
-0.503 & -0.244 & -0.25 \\
-0.682 & -0.062 & -0.174 \\
-0.528 & -0.581 & -0.094 \\
0.177 & 0.534 & -0.658 \\
0.805 & 0.409 & -0.243 \\
-0.149 & -0.141 & 0.979 \\
\hline & &
\end{tabular}




\begin{tabular}{ccc}
\hline 0.020 & 0.135 & 0.326 \\
-0.011 & 0.608 & -0.074 \\
0.449 & -0.544 & -0.048 \\
0.428 & -0.015 & 0.664 \\
0.478 & -0.72 & 0.059 \\
-0.459 & -0.603 & -0.549 \\
-0.545 & 0.589 & -0.597 \\
-0.15 & -0.319 & -0.031 \\
-0.364 & -0.576 & 0.19 \\
0.171 & -0.554 & -0.537 \\
0.24 & -0.364 & -0.748 \\
-0.46 & 0.189 & 0.792 \\
-0.466 & -0.752 & 0.466 \\
-0.321 & -0.012 & -0.148 \\
0.222 & -0.708 & 0.274 \\
0.399 & 0.741 & 0.206 \\
-0.318 & 0.196 & -0.858 \\
-0.99 & -0.137 & -0.044 \\
-0.862 & 0.311 & -0.189 \\
-0.458 & 0.878 & 0.14 \\
-0.719 & 0.299 & 0.628 \\
\hline & &
\end{tabular}

72 diffusion encoding directions for CHARMED at $1.5 \mathrm{~mm}$ iso

\begin{tabular}{ccc}
\hline $\mathbf{X}$ & $\mathbf{Y}$ & $\mathbf{Z}$ \\
\hline 0.103805 & 0.179404 & -0.286308 \\
0.182447 & -0.281857 & -0.110467 \\
0.235783 & -0.429501 & 0.0996770 \\
-0.456572 & -0.0950230 & -0.180313 \\
0.308745 & -0.0624470 & 0.525081 \\
-0.0178300 & -0.484999 & -0.373352 \\
-0.216332 & -0.119436 & 0.662522 \\
0.301178 & -0.407204 & -0.493434 \\
0.465132 & 0.507581 & -0.388605 \\
\hline
\end{tabular}




\begin{tabular}{|c|c|c|}
\hline 0.0879780 & -0.423019 & 0.662053 \\
\hline-0.0610800 & -0.709542 & 0.492767 \\
\hline 0.544096 & 0.308735 & -0.598867 \\
\hline 0.197302 & -0.603463 & 0.680860 \\
\hline 0.310426 & 0.747588 & -0.459799 \\
\hline-0.540863 & -0.201780 & 0.816549 \\
\hline 0.942777 & 0.0905740 & -0.320886 \\
\hline 0.265519 & 0.258426 & -0.335732 \\
\hline 0.0389080 & -0.271238 & -0.418230 \\
\hline-0.539248 & -0.287081 & -0.0415850 \\
\hline-0.329252 & -0.0326870 & 0.515226 \\
\hline-0.604605 & 0.364485 & -0.0400460 \\
\hline-0.519031 & -0.341534 & -0.337581 \\
\hline-0.605324 & 0.488398 & -0.141599 \\
\hline-0.566302 & 0.0738960 & 0.546664 \\
\hline 0.548950 & 0.366850 & 0.560424 \\
\hline-0.0254120 & -0.466277 & -0.729342 \\
\hline-0.507955 & -0.764412 & -0.155956 \\
\hline 0.727106 & 0.247338 & 0.526124 \\
\hline-0.0828330 & 0.0708890 & -0.994039 \\
\hline 0.627483 & -0.161116 & 0.761779 \\
\hline 0.498950 & -0.354431 & -0.0189900 \\
\hline-0.0442740 & -0.549849 & 0.265781 \\
\hline 0.0148510 & -0.605602 & 0.364727 \\
\hline-0.378345 & 0.133650 & -0.582231 \\
\hline 0.420231 & 0.587309 & 0.321675 \\
\hline 0.544607 & -0.0168270 & 0.572817 \\
\hline 0.532067 & -0.660821 & 0.173839 \\
\hline 0.0573440 & 0.111400 & -0.856914 \\
\hline-0.290069 & 0.0163300 & -0.884454 \\
\hline 0.723546 & -0.257273 & -0.526269 \\
\hline 0.399032 & -0.916106 & -0.0390150 \\
\hline 0.938767 & -0.330288 & 0.0981140 \\
\hline-0.0800930 & -0.679664 & -0.177882 \\
\hline-0.619423 & -0.246048 & 0.236168 \\
\hline 0.780942 & 0.122437 & 0.0117770 \\
\hline
\end{tabular}




\begin{tabular}{ccc}
\hline-0.0133610 & 0.781851 & -0.116319 \\
0.497188 & -0.297249 & 0.643775 \\
-0.0642680 & -0.853132 & -0.134297 \\
0.689479 & -0.448251 & 0.436298 \\
0.580453 & -0.709452 & -0.162539 \\
-0.602139 & -0.769475 & -0.212925 \\
0.321132 & -0.386113 & -0.864749 \\
-0.294705 & 0.618002 & 0.395250 \\
0.0111780 & 0.223197 & 0.758326 \\
0.616337 & 0.607401 & -0.0345420 \\
0.773881 & -0.153808 & -0.357004 \\
-0.181394 & -0.677043 & -0.612679 \\
0.236118 & -0.393862 & -0.809807 \\
-0.155790 & -0.963498 & 0.217716 \\
0.00713400 & -0.862253 & -0.506427 \\
0.448457 & -0.595170 & -0.441202 \\
0.846291 & 0.00496500 & 0.183757 \\
0.791781 & 0.410438 & -0.267002 \\
0.927321 & -0.0792730 & 0.0214100 \\
-0.899261 & -0.260722 & -0.351218 \\
-0.00311800 & -0.661547 & 0.749897 \\
0.104713 & -0.925032 & 0.00417600 \\
0.384607 & 0.220639 & -0.818573 \\
0.577848 & -0.672083 & 0.463029 \\
-0.396977 & -0.483951 & -0.779873 \\
0.700807 & -0.547477 & -0.457316 \\
-0.666456 & -0.631395 & 0.396455 \\
\hline & & \\
\hline
\end{tabular}

108 diffusion encoding directions for CHARMED at $1.3 \mathrm{~mm}$ iso

\begin{tabular}{ccc}
\hline $\mathbf{X}$ & $\mathbf{Y}$ & $\mathbf{Z}$ \\
\hline 0.104340 & -0.0830180 & 0.327345 \\
-0.335446 & 0.0153650 & 0.110331 \\
0.0399290 & 0.343324 & 0.0739450 \\
\hline
\end{tabular}




\begin{tabular}{|c|c|c|}
\hline 0.0426490 & -0.450937 & 0.211748 \\
\hline 0.0584580 & 0.394872 & 0.301097 \\
\hline-0.342334 & 0.0444430 & -0.361707 \\
\hline 0.595422 & -0.0671390 & -0.126091 \\
\hline-0.403559 & -0.368882 & 0.275680 \\
\hline-0.0444720 & 0.229852 & -0.565795 \\
\hline-0.400014 & -0.234230 & -0.533971 \\
\hline 0.679312 & 0.00489100 & 0.196243 \\
\hline-0.101107 & 0.521795 & 0.466376 \\
\hline-0.702563 & 0.159200 & 0.325669 \\
\hline 0.733683 & 0.294407 & -0.00579700 \\
\hline 0.253340 & -0.217934 & -0.716466 \\
\hline-0.0133510 & 0.626066 & -0.598216 \\
\hline-0.449797 & -0.250574 & 0.696344 \\
\hline 0.418486 & -0.477834 & -0.588680 \\
\hline 0.665424 & 0.520950 & -0.390497 \\
\hline-0.382246 & -0.828332 & 0.185530 \\
\hline 0.755804 & -0.0936030 & 0.535411 \\
\hline-0.259315 & -0.818290 & -0.512988 \\
\hline 0.236270 & 0.905715 & -0.351932 \\
\hline 0.732117 & -0.668463 & 0.131004 \\
\hline 0.411006 & -0.262911 & -0.109325 \\
\hline-0.172976 & -0.0472930 & 0.466736 \\
\hline 0.420277 & 0.264728 & -0.0573250 \\
\hline-0.484337 & -0.291089 & -0.235834 \\
\hline 0.344799 & -0.484302 & -0.146626 \\
\hline-0.454453 & 0.203782 & -0.356201 \\
\hline 0.366249 & -0.129704 & -0.590795 \\
\hline-0.529925 & 0.460980 & 0.0817120 \\
\hline 0.465482 & 0.471947 & -0.246155 \\
\hline 0.188920 & 0.540992 & 0.544644 \\
\hline-0.260660 & -0.0706030 & -0.743015 \\
\hline-0.344598 & 0.673021 & -0.230856 \\
\hline-0.480165 & 0.716533 & 0.0775980 \\
\hline-0.00939100 & -0.853137 & 0.148558 \\
\hline-0.403326 & -0.464151 & -0.609830 \\
\hline
\end{tabular}




\begin{tabular}{lll}
\hline 0.236702 & 0.107261 & -0.893943 \\
0.211668 & -0.834576 & -0.354042 \\
0.902595 & 0.223082 & -0.0471490 \\
0.695908 & 0.585722 & 0.415503 \\
-0.305682 & -0.362566 & -0.880400 \\
-0.279678 & 0.147916 & -0.948631 \\
0.00453000 & -0.591443 & 0.158456 \\
-0.103344 & -0.435909 & -0.417417 \\
0.255549 & -0.129885 & -0.541071 \\
-0.423128 & 0.361656 & -0.436083 \\
0.345367 & 0.579725 & 0.211282 \\
-0.107263 & 0.697575 & -0.0434040 \\
0.406911 & 0.675220 & 0.0591750 \\
-0.625244 & -0.200292 & -0.440401 \\
-0.719287 & 0.284790 & -0.162854 \\
-0.421658 & 0.0824820 & -0.751932 \\
-0.803508 & 0.322686 & 0.0157630 \\
-0.696213 & 0.152179 & 0.492066 \\
-0.547557 & -0.358544 & -0.662038 \\
-0.315771 & -0.801792 & -0.352258 \\
0.256820 & 0.547947 & -0.707435 \\
0.781016 & 0.611879 & -0.124970 \\
0.895645 & 0.201661 & -0.396424 \\
-0.561519 & 0.485683 & -0.669931 \\
-0.0426580 & 0.0666170 & -0.702668 \\
0.0732690 & 0.504501 & -0.490011 \\
0.650483 & 0.0408060 & -0.274238 \\
-0.0742490 & 0.767480 & 0.174534 \\
-0.400464 & 0.357202 & -0.580548 \\
0.134401 & 0.296578 & -0.720401 \\
-0.436129 & -0.725865 & -0.181416 \\
\hline & -0.0916960 & -0.419443 \\
\hline & -0.776053 & -0.381176 \\
\hline
\end{tabular}




\begin{tabular}{|c|c|c|}
\hline 0.305433 & -0.951508 & 0.0366470 \\
\hline 0.966320 & 0.185774 & 0.178081 \\
\hline 0.142178 & -0.545815 & -0.825755 \\
\hline 0.159184 & 0.672894 & -0.383241 \\
\hline 0.559623 & 0.294998 & -0.474129 \\
\hline-0.445859 & 0.548159 & 0.354585 \\
\hline-0.792467 & -0.249417 & 0.244515 \\
\hline 0.0251150 & -0.317077 & -0.805501 \\
\hline-0.474910 & -0.643160 & 0.332875 \\
\hline 0.715313 & 0.544286 & 0.242376 \\
\hline 0.472624 & -0.360327 & -0.716559 \\
\hline 0.370274 & -0.250874 & 0.816472 \\
\hline 0.00702100 & 0.532351 & -0.846495 \\
\hline 0.399919 & 0.914793 & 0.0567370 \\
\hline 0.200887 & -0.880425 & -0.429531 \\
\hline-0.492069 & 0.571359 & -0.425931 \\
\hline-0.769512 & -0.330813 & -0.220033 \\
\hline 0.0121590 & 0.183414 & -0.846293 \\
\hline 0.705241 & 0.0917130 & -0.600742 \\
\hline 0.171161 & -0.687710 & 0.603677 \\
\hline 0.790586 & -0.350087 & -0.345078 \\
\hline 0.663604 & -0.649611 & -0.370992 \\
\hline 0.953327 & -0.238398 & -0.185294 \\
\hline 0.883655 & -0.262400 & 0.387686 \\
\hline 0.0250960 & -0.593991 & -0.716388 \\
\hline-0.147183 & -0.189372 & -0.899523 \\
\hline 0.579489 & -0.723191 & -0.0886190 \\
\hline-0.230343 & 0.836712 & -0.496846 \\
\hline 0.707449 & 0.137241 & 0.693311 \\
\hline 0.635503 & -0.324007 & -0.700824 \\
\hline 0.604460 & 0.165271 & -0.779303 \\
\hline 0.204704 & -0.0417610 & -0.977933 \\
\hline 0.482037 & 0.607478 & -0.631356 \\
\hline
\end{tabular}


Chapter 5 
Chapter 6

Summary and future directions 
The studies reported and discussed in this thesis investigate the structural architecture and the anatomical connectivity of the human brain at different spatial scales. The macroscopic, mesoscopic and microscopic organization of brain connectivity and anatomy is probed with diffusion Magnetic Resonance Imaging (dMRI) and tractography techniques combined with histological staining approaches and mathematical network analysis. The described research contributes both to dMRI and tractography methodology and basic knowledge of human neuroanatomy.

\section{Macroscopic brain organization}

Given the large variety of dMRI-based tractography algorithms available (Basser et al., 2000; Behrens et al., 2007; Iturria-Medina et al., 2007; Parker et al., 2003; Sotiropoulos et al., 2010; Wedeen et al., 2008), chapter 2 aims at evaluating their differences when reconstructing graph representations of the human macroscale connectome. We reconstruct the whole cortico-cortical connectivity matrix using a variety of tractography parameters, such as angular and fractional anisotropy thresholds, seeding strategies and six different algorithmic classes. We evaluate the effects of these variations on scalar graph theoretical indices (for a review, see: Rubinov and Sporns, 2010). We show that density and other indices such as small-worldness are greatly affected by the choice of tractography algorithm and the value of its user-set parameters. Furthermore, we address the issue of quality control of the whole connectivity matrix (Cammoun et al., 2012; Owen et al., 2013) by introducing the tract specific density coefficient (TSDC). This index provides a reliable quantitative measure of the quality of reconstruction of a known tract, assessing the sensitivity of a tractography approach. Moreover, we show it can be used to detect false negative results within the entire connectivity matrix, assessing its specificity. Finally we show that assessing the trade-off between sensitivity and specificity can assist in objectively setting probabilistic thresholds in tractography. Based on 
TSDC assessment we conclude that more sophisticated diffusion models, capable of reconstructing multiple fiber directions, and probabilistic or global tractography approaches should be preferred when performing structural connectomics. This is an important recommendation since connectomes are currently mostly obtained from diffusion tensor imaging (DTI) and deterministic streamline tractography. By applying the TSDC method developed in the chapter, newly developed fiber tracking algorithms can be evaluated with respect to both their sensitivity and specificity. Finally, quality control of connectomes obtained from large population studies can also be implemented using the proposed TSDC framework.

Diffusion MRI data quality keeps improving very rapidly and is currently moving towards mesoscopic resolutions. Voxel resolutions of $1 \mathrm{~mm}^{3}$ are achievable using a clinical 3T machine as illustrated in figure 1 , while data at sub-millimeter resolution can be acquired using ultrahigh field MRI scanners (Heidemann et al., 2012). Tractography approaches will need to be carefully validated and possibly improved before being applied to mesoscale dMRI data. Future work can aim at using the TSDC framework in assessing the improvements in connectome reconstruction possible when using this much higher resolution.

In chapter 3 we used the methods and parameters for connectome mapping recommended in chapter 2 to compare the macroscale brain connectivity of two different species, namely human and macaque. We propose a rigorous and quantitative framework for anatomical comparative studies. This is based, on the one hand, on a database of whole brain tracer injection results for the macaque brain and, on the other hand, on in-vivo connectome mapping using dMRI tractography for the human brain. We directly compare the structural pattern and the topological properties of the two species using network analysis approaches. On the whole, the brain of humans and macaques show a very high degree of similarity in their macroscale connectome. 


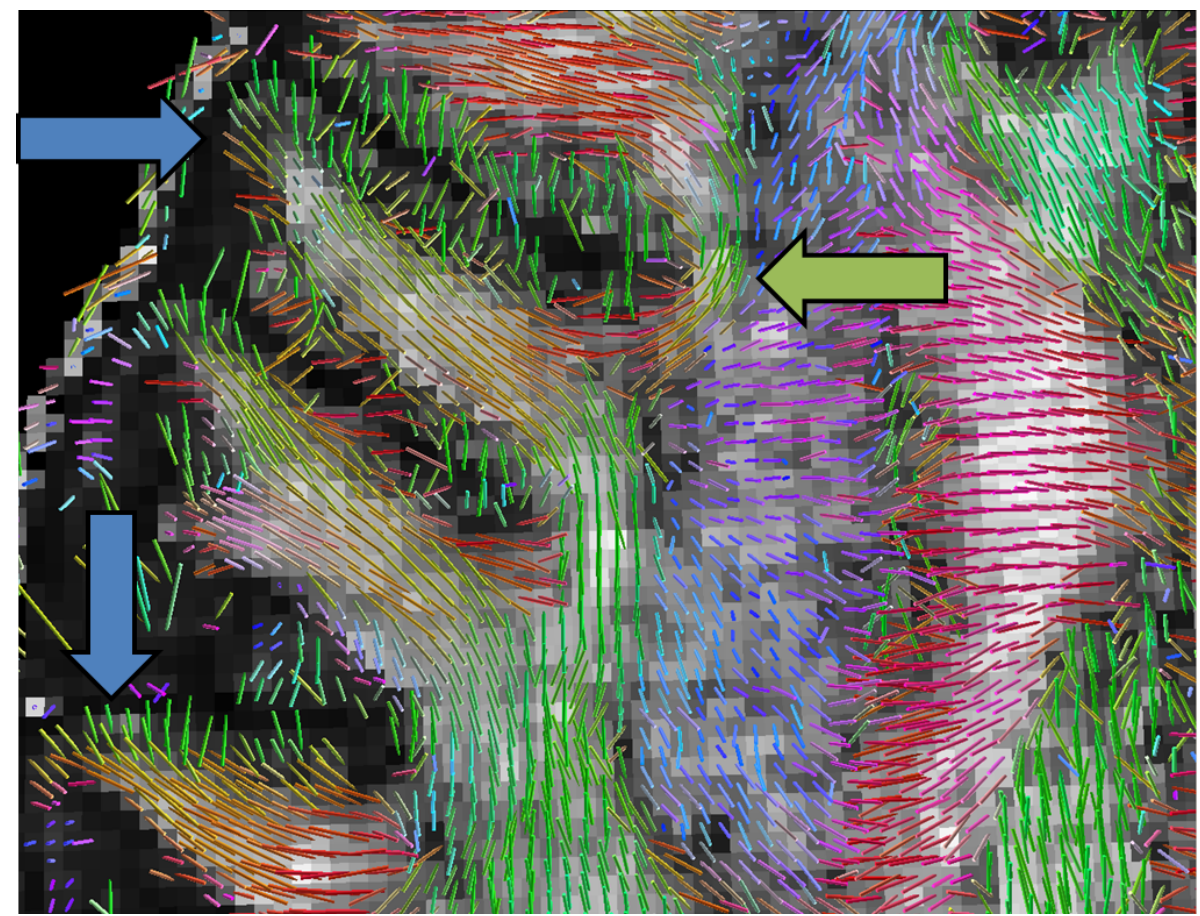

Figure 1: Major eigenvector from standard DTI analysis of $1 \mathrm{~mm}^{3}$ isotropic data superimposed on an axial slice of fractional anisotropy. Data was acquired using a 3T MRI scanner and a b-value of $1000 \mathrm{~s} / \mathrm{mm}^{2}$. Blue arrows highlight radial cortical fiber insertions within gray matter, while the green arrow points at a short-association fiber connecting to adjacent gyri.

Specifically, a set of frontal, occipital and temporal regions exhibit a significant overlap of their whole brain connectivity across the species. The most important differences are present in the connectivity of parietal and cingulate regions. Furthermore, we identify a common structural backbone that could have been preserved during mammalian evolution. Our comparative analysis demonstrates that the regions forming a so-called rich club (van den Heuvel and Sporns, 2011) exhibit a high and significant overlap in 14 out of 20 regions. These mainly involve regions located in the frontal, parietal, cingulate and insular cortex. We conclude that the human and macaque brain as a whole are similarly wired. Moreover, we offer a rigorous and quantitative 
translational bridge between macaque and human research. This framework will be very useful in the field of comparative neuroanatomy especially since recent developments have provided the scientific community with better tracers. The combination of our quantitative comparative approach with better tracing techniques capable of identifying weak and directed connections (Markov et al., 2014) and high-resolution dMRI data (Fig. 1) will help to further improve humanmacaque brain connectivity comparisons and translation.

\section{Mesoscopic brain organization}

In chapter 4 we shift perspective from the macroscale organization of brain connectivity through white matter to the mesoscopic organization of the neocortex, focusing on its laminar organization. It has recently been shown that dMRI is capable of describing the intra and interlaminar connectivity within post mortem tissue samples (Kleinnijenhuis et al., 2013; Leuze et al., 2014). We use dMRI of a human post mortem tissue sample containing motor and premotor cortex acquired at ultrahigh field (9.4T). The high spatial resolution (340 $\mu \mathrm{m}$ isotropic) and angular resolution (60 diffusion directions) of the acquired data enabled automatic delineation of several distinct cortical layers in the entire span of the sample. Our results and their validation based on histology show that dMRI can probe layer-specific intracortical fiber organization and classify architecturally distinct cortical areas. This work establishes methodological requirements on classification of gray matter architecture and microcircuitry by dMRI contrast and paves the way for non-invasive, in vivo human histology. As such it shows that highresolution diffusion MRI not only reveals the edges (i.e. white matter projections) of the human macroscale connectome but also the respective nodes (i.e. architecturally distinct gray matter areas). 


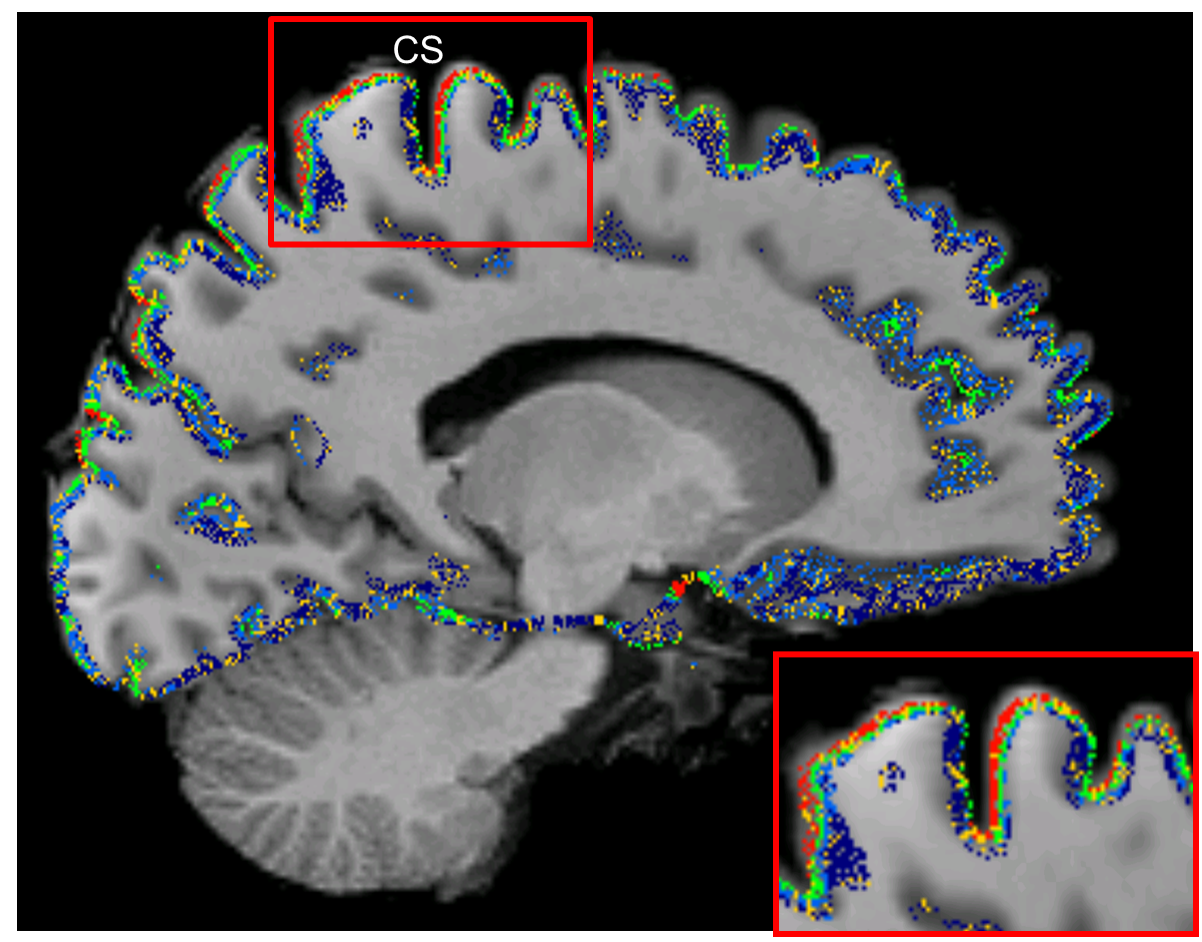

Figure 2: In vivo whole brain dMRI-based automatic cortical layer segmentation superimposed to a T1-weighted sagittal slice. Data was acquired using a 3T MRI scanner and a b-value of $2000 \mathrm{~s} / \mathrm{mm}^{2}$. Both dMRI and T1 data have a voxel resolution of $1 \mathrm{~mm}^{3}$. Three relatively depth-specific clusters (red, green and light blue) are identified. CS: central sulcus.

The steadily increasing quality and resolution of dMRI data can be utilized not only in white matter, but also to probe the mesoscale organization within gray matter. Figure 2 shows results of applying the developed cortical layer classification technique to $1 \mathrm{~mm}$ isotropic invivo dMRI data. Future work can continue to develop and apply the developed techniques to the study of larger expanses of cortex ex vivo and in vivo and help establish dMRI based histology approaches. Using modern neuroanatomical techniques it is possible to unravel the structural basis of brain functions such as language (Amunts et al., 2010) and motor control (Geyer et al., 1996). DMRI-based histology could be 
capable of achieving similar results in vivo and in larger population studies.

\section{Microscopic brain organization}

In chapter 5 we focus on the microscopic organization of white matter. The estimation of the volume fraction of the hindered (extra-axonal) and restricted (intra-axonal) water compartments using the composite hindered and restricted model of diffusion (CHARMED; Assaf and Basser, 2005) usually requires very long acquisition times. This is because acquisitions with multiple high $b$-values and high angular resolution are needed. We combine protocol optimization, simultaneous multi-slice/multi-band imaging (SMS/MB; Moeller et al., 2010) and a high amplitude, gradient set to enable short-time high-resolution CHARMED acquisitions. We show that short-time high-resolution CHARMED acquisitions are feasible when using the three methods combined and provide optimized reference protocols. Furthermore, we evaluate resolution and partial volume effects for the microstructurespecific restricted fraction (FR) index. We show that at higher resolution the estimated FR will tend to be higher. These findings show that wholebrain microstructural mapping is feasible at high spatial resolution in clinically feasible times. Moreover, they highlight the issue of comparability between different studies performed at different resolutions. Our study should provide the basis to further objective quantification of microstructural indices and their dependency on data acquisition parameters (e.g. spatial resolution).

\section{Future directions}

Since dMRI data resolution keeps improving (Figs. 1-2) and both the accuracy and the precision of microstructural mapping techniques are constantly increasing, future studies will need to take into account the 
different levels in which the brain is organized more explicitly. The work in this thesis illustrates how combination of acquisition modalities and analysis frameworks can support this endeavor. Macro-, meso- and microstructural organizational principles will need to be combined in the same models of the brain. Multimodal data acquired on the same subject or post mortem tissue at different scales (e.g. MRI and light microscopy) will need to be fused and interpreted together rather than kept as standalone findings. Further developments in this direction can deliver new MRI-based atlas resources with a high translation potential to in vivo MRI imaging. Furthermore, when combined with genetic, molecular, cyto- and myeloarchitectural information these new datasets could help the creation of detailed multimodal atlases (Amunts et al., 2014; Toga et al., 2006).

Technical advancements from the world of microscopy are constantly increasing both in the level of detail and the field of view which can be investigated. Recent efforts have already shown the potential of classical cytoarchitectonic staining of an entire brain at the very high resolution of $20 \mu \mathrm{m}$ (Amunts et al., 2013) with more individual datasets currently underway. Combining such maps with observer independent techniques to demarcate architecturally different cortical areas (Schleicher et al., 1999) will allow identifying new common structural principles which are shared between individuals and which could not be described in earlier works based on single subjects (Brodmann, 1909; Talairach and Tournoux, 1988). Moreover, it has been shown that three-dimensional structural connections can be investigated in 3D in larger tissue samples after having made the tissue transparent with optical clearing techniques (Chung and Deisseroth, 2013).

On the other hand, validation of dMRI-based techniques is still an important issue. The combination of whole brain post mortem findings obtained from techniques such as polarized light imaging (PLI; Axer et al., 2011) with tractography results might represent a very good 
way to address this. Both techniques in principle allow for a whole brain three-dimensional reconstruction of fiber tracts. Here it should be noted that both techniques, be it at different intrinsic resolutions, have the same basic tractography problem to solve, starting from local fiber directions. At the microstructural mapping side, there is also the need to improve the sensitivity and the specificity of estimated microstructural indices and reconstructed axonal pathways (Bells et al., 2011).

Finally, consortium projects including large population studies have recently started mapping structural and functional macroscale connectomes of adult human brains (Sotiropoulos et al., 2013; Van Essen and Ugurbil, 2012) and newborns (http://www.developingconnectome.org/) at the population level and at very high resolution. Making these data available to the whole scientific community must become a fundamental prior to any future study aimed at mapping the structural architecture of the brain.

We hope that the empirical work presented in this thesis will represent a step forward in the field of in vivo neuroanatomy and that it will constitute the basis on which the aforementioned future directions can be further developed. 


\section{References}

Amunts, K., Hawrylycz, M.J., Van Essen, D.C., Van Horn, J.D., Harel, N., Poline, J.B., De Martino, F., Bjaalie, J.G., Dehaene-Lambertz, G., Dehaene, S., Valdes-Sosa, P., Thirion, B., Zilles, K., Hill, S.L., Abrams, M.B., Tass, P.A., Vanduffel, W., Evans, A.C., Eickhoff, S.B., 2014. Interoperable atlases of the human brain. Neuroimage.

Amunts, K., Lenzen, M., Friederici, A.D., Schleicher, A., Morosan, P., PalomeroGallagher, N., Zilles, K., 2010. Broca's region: novel organizational principles and multiple receptor mapping. PLoS Biol 8.

Amunts, K., Lepage, C., Borgeat, L., Mohlberg, H., Dickscheid, T., Rousseau, M.E., Bludau, S., Bazin, P.L., Lewis, L.B., Oros-Peusquens, A.M., Shah, N.J., Lippert, T., Zilles, K., Evans, A.C., 2013. BigBrain: an ultrahigh-resolution 3D human brain model. Science 340, 1472-1475.

Assaf, Y., Basser, P.J., 2005. Composite hindered and restricted model of diffusion (CHARMED) MR imaging of the human brain. Neuroimage 27, 48-58.

Axer, M., Grassel, D., Kleiner, M., Dammers, J., Dickscheid, T., Reckfort, J., Hutz, T., Eiben, B., Pietrzyk, U., Zilles, K., Amunts, K., 2011. High-resolution fiber tract reconstruction in the human brain by means of three-dimensional polarized light imaging. Front Neuroinform 5, 34.

Basser, P.J., Pajevic, S., Pierpaoli, C., Duda, J., Aldroubi, A., 2000. In vivo fiber tractography using DT-MRI data. Magn Reson Med 44, 625-632.

Behrens, T.E., Berg, H.J., Jbabdi, S., Rushworth, M.F., Woolrich, M.W., 2007. Probabilistic diffusion tractography with multiple fibre orientations: What can we gain? Neuroimage 34, 144-155.

Bells, S., Cercignani, M., Deoni, S., Assaf, Y., Pasternak, O., Evans, C.J., Leemans, A., Jones, D.K., 2011. Tractometry: comprehensive multi-modal quantitative assessment of white matter along specific tracts. Proc. Int. Soc. Magn. Res. Med., Montreal.

Brodmann, K., 1909. Vergleichende Lokalisationslehre der Grosshirnrinde in ihren Prinzipien dargestellt auf Grund des Zellenbaues.

Cammoun, L., Gigandet, X., Meskaldji, D., Thiran, J.P., Sporns, O., Do, K.Q., Maeder, P., Meuli, R., Hagmann, P., 2012. Mapping the human connectome at multiple scales with diffusion spectrum MRI. J Neurosci Methods 203, 386-397.

Chung, K., Deisseroth, K., 2013. CLARITY for mapping the nervous system. Nat Methods 10, 508-513.

Geyer, S., Ledberg, A., Schleicher, A., Kinomura, S., Schormann, T., Burgel, U., Klingberg, T., Larsson, J., Zilles, K., Roland, P.E., 1996. Two different areas within the primary motor cortex of man. Nature 382, 805-807.

Heidemann, R.M., Anwander, A., Feiweier, T., Knosche, T.R., Turner, R., 2012. k-space and q-space: combining ultra-high spatial and angular resolution in diffusion imaging using ZOOPPA at 7 T. Neuroimage 60, 967-978. 
Iturria-Medina, Y., Canales-Rodriguez, E.J., Melie-Garcia, L., Valdes-Hernandez, P.A., Martinez-Montes, E., Aleman-Gomez, Y., Sanchez-Bornot, J.M., 2007. Characterizing brain anatomical connections using diffusion weighted MRI and graph theory. Neuroimage 36, 645-660.

Kleinnijenhuis, M., Zerbi, V., Kusters, B., Slump, C.H., Barth, M., van Cappellen van Walsum, A.M., 2013. Layer-specific diffusion weighted imaging in human primary visual cortex in vitro. Cortex 49, 2569-2582.

Leuze, C.W., Anwander, A., Bazin, P.L., Dhital, B., Stuber, C., Reimann, K., Geyer, S., Turner, R., 2014. Layer-specific intracortical connectivity revealed with diffusion MRI. Cerebral Cortex 24, 328-339.

Markov, N.T., Ercsey-Ravasz, M.M., Ribeiro Gomes, A.R., Lamy, C., Magrou, L., Vezoli, J., Misery, P., Falchier, A., Quilodran, R., Gariel, M.A., Sallet, J., Gamanut, R., Huissoud, C., Clavagnier, S., Giroud, P., Sappey-Marinier, D., Barone, P., Dehay, C., Toroczkai, Z., Knoblauch, K., Van Essen, D.C., Kennedy, H., 2014. A weighted and directed interareal connectivity matrix for macaque cerebral cortex. Cereb Cortex 24, 17-36.

Moeller, S., Yacoub, E., Olman, C.A., Auerbach, E., Strupp, J., Harel, N., Ugurbil, K., 2010. Multiband multislice GE-EPI at 7 tesla, with 16-fold acceleration using partial parallel imaging with application to high spatial and temporal whole-brain fMRI. Magn Reson Med 63, 1144-1153.

Owen, J.P., Ziv, E., Bukshpun, P., Pojman, N., Wakahiro, M., Berman, J.I., Roberts, T.P., Friedman, E.J., Sherr, E.H., Mukherjee, P., 2013. Test-retest reliability of computational network measurements derived from the structural connectome of the human brain. Brain Connect 3, 160-176.

Parker, G.J., Haroon, H.A., Wheeler-Kingshott, C.A., 2003. A framework for a streamline-based probabilistic index of connectivity (PICo) using a structural interpretation of MRI diffusion measurements. J Magn Reson Imaging 18, 242-254.

Rubinov, M., Sporns, O., 2010. Complex network measures of brain connectivity: uses and interpretations. Neuroimage 52, 1059-1069.

Schleicher, A., Amunts, K., Geyer, S., Morosan, P., Zilles, K., 1999. Observerindependent method for microstructural parcellation of cerebral cortex: A quantitative approach to cytoarchitectonics. Neuroimage 9, 165-177.

Sotiropoulos, S.N., Bai, L., Morgan, P.S., Constantinescu, C.S., Tench, C.R., 2010. Brain tractography using Q-ball imaging and graph theory: Improved connectivities through fibre crossings via a model-based approach. Neuroimage 49, 2444-2456.

Sotiropoulos, S.N., Jbabdi, S., Xu, J., Andersson, J.L., Moeller, S., Auerbach, E.J., Glasser, M.F., Hernandez, M., Sapiro, G., Jenkinson, M., Feinberg, D.A., Yacoub, E., Lenglet, C., Van Essen, D.C., Ugurbil, K., Behrens, T.E., Consortium, W.U.-M.H., 2013. Advances in diffusion MRI acquisition and processing in the Human Connectome Project. Neuroimage 80, 125-143. 
Talairach, J., Tournoux, P., 1988. Co-planar stereotaxic atlas of the human brain : 3dimensional proportional system : an approach to cerebral imaging. Georg Thieme, Stuttgart; New York.

Toga, A.W., Thompson, P.M., Mori, S., Amunts, K., Zilles, K., 2006. Towards multimodal atlases of the human brain. Nat Rev Neurosci 7, 952-966.

van den Heuvel, M.P., Sporns, O., 2011. Rich-club organization of the human connectome. J Neurosci 31, 15775-15786.

Van Essen, D.C., Ugurbil, K., 2012. The future of the human connectome. Neuroimage 62, 1299-1310.

Wedeen, V.J., Wang, R.P., Schmahmann, J.D., Benner, T., Tseng, W.Y., Dai, G., Pandya, D.N., Hagmann, P., D'Arceuil, H., de Crespigny, A.J., 2008. Diffusion spectrum magnetic resonance imaging (DSI) tractography of crossing fibers. Neuroimage 41, 1267-1277. 


\section{Appendix}

Knowledge valorization 


\section{Social relevance}

The average human brain weighs $1.5 \mathrm{~kg}$ and contains about 100 billion neurons, each of which is connected to thousands of other neurons. To precisely map such a complicated network represents a fundamental endeavor for both the scientific and the clinical community that will have huge impact on our society (Van Essen and Ugurbil, 2012).

With this thesis we have contributed to the challenge of mapping the brain connectivity profile at three different scales: the macroscopic of millimeters, the mesoscopic of hundreds of micrometers and the microscopic of single micrometers. We address several fundamental research questions related to the anatomy of the brain and the mapping of its connections, improving existing techniques and implementing new and efficient ones. Even though the main focus of this thesis is on unraveling some of the basic principles of the architectural backbone of the central nervous system, there are several outcomes which both society and industry can benefit from. These outcomes are fourfold and pass on their knowledge for utilization in varying degrees of directness and on different timescales. First, all the scientific projects which are described and discussed in the different chapters are inherently very multidisciplinary. Knowledge drawn from the latest developments in mathematical optimization techniques, parallel computer algorithm design and complex network analysis is used to achieve innovations in the field of life sciences. Second, the methodological tools developed and fundamental knowledge gained in the context of this thesis come to the benefit of large research initiatives started in recent years, which focus on gathering human brain connectivity data in order to provide fundamental knowledge for societal utilization. Third, clinical research collaborations performed in the context of this thesis on Multiple sclerosis and Parkinson's disease, illustrate how knowledge is already being applied close to the clinical practice. Lastly, all the knowledge and tools derived from this thesis can be used to improve current educational programs. 
A multidisciplinary approach is fundamental to unravel the structural complexity of the brain. It is therefore very important that the scientific and clinical communities join their forces and actively collaborate to cure those diseases which are primarily targeting the brain and that represent one of the major sources of cost for the national health care systems. This will result in an improved quality of life of the patients and a reduction of the costs associated with chronic diseases. This project's developments will boost in practical availability of advanced modeling techniques for medical and fundamental neuroscience research. This also includes the new fields of connectomics (Sporns et al., 2005) and computational modeling of human brain dynamics which will greatly benefit from better tractography and information on conduction velocity and information-carrying capacity implied by axonal density and diameter measures.

\section{Target group}

The results of this thesis are relevant not only to the neuroscientific community, but also to medical doctors which deal with neurodegenerative diseases. The methods implemented in this thesis have the potential to improve both diagnosis and treatment planning of diseases such as Alzheimer's disease, Parkinson's disease and Multiple Sclerosis.

Moreover, as this thesis contains a lot of methodological advancements, biomedical engineers and bio-informatics that deal with MRI data analysis will greatly benefit from the thorough explanation of the methods which is contained in every chapter.

\section{Activities and products}

All the algorithms which have been implemented in this thesis will be made freely available to the community by setting up an open-source 
parallelized microstructure modeling and tractography toolbox that interfaces with existing tools such as Camino (Cook et al., 2006), FSLFDT (Behrens et al., 2007), ExploreDTI (Leemans et al., 2009) and ConnectomeViewer (Gerhard et al., 2011). It will be written in MATLAB (for prototyping developments) and python using numpy \& scipy (for numerical routines), nipy \& nipype (for MRI image file access and interfacing to other tools) and pyOpenCL \& pyCUDA (for CPU/GPU parallelization). This will make efficient routines for very large-scale microstructure model optimization and tractography available to the community for usage and further development. This is an urgent requirement because continued development and widespread use of microstructure compartment models and global tractography is currently inhibited by the tremendous large-scale optimization challenges they pose leading to excessively long computation times.

Another great outcome of the present thesis is related to the field of education. Comparative neuroanatomy (see chapter 3) together with the possibility of reconstructing very accurately and precisely in three dimensions single axonal bundles of thousands of subject open up a whole new set of tools which can be used by future generations of life sciences' students. New MRI-based atlases can be developed at resolutions which are getting very close to those used in current medical atlases and mostly derived from post mortem analyses. Thanks to modern 3D printers, then, very good models of the brain architecture can be produced and diseases which require surgical intervention can be modeled in such three dimensional reproductions on which neurosurgeons can train prior to the real surgery.

\section{Innovation}

Over the past years, new sophisticated and biologically plausible diffusion MRI (dMRI)-based techniques have been introduced. High angular resolution diffusion imaging (HARDI) has overcome the 
intrinsic limitation of diffusion tensor imaging (DTI), namely that of being only capable of reconstructing a single fiber direction in every image voxel. This has allowed researchers to model more complex structural configurations, accounting for multiple fibers present in single voxels which might be crossing or kissing. Connectomes produced using such techniques together with improved tractography algorithms will obviously be more realistic and complete. This point has been clearly elucidated in the second chapter of the present thesis. There we show the different consequences of choosing a certain tractography algorithm for connectome reconstruction and introduce a quality assurance framework. Furthermore, it has recently been shown that $\mathrm{dMRI}$ can be used not only to map the connections within white matter, but also inside gray matter, as also investigated in this thesis in chapter 4. This opens new possibilities to study diseases related not only to white matter but also to the cortex. Lastly, a new group of techniques allows researchers and clinicians to estimate axonal packing densities and diameters, as investigated also in this thesis in chapter 5 . The correct mapping of these biological features can unravel microscopic details of the brain in vivo. Future drug development and clinical trials can immensely benefit from these possibilities as it becomes possible to see how they affect different microstructural compartments such as intracellular or extracellular space.

The methodological tools described previously and implemented in this thesis will help analyzing the data acquired by large research initiatives which have recently started. The United States National Institute of Health (NIH) has funded a thirty million dollar grant over five years to map the human macroscale connectome (i.e. the whole brain cortico-cortical connectivity map at a scale of millimeters). This grant was assigned to two consortia, one led by Washington University and the University of Minnesota (http://humanconnectome.org/about/pressroom/tag/brain-initiative/) and the other led by Harvard and the Massachussets General Hospital 
(http://www.humanconnectomeproject.org/about/). The WashU/MU project aims at reconstructing the structural and functional connectome of 1200 healthy individuals using diffusion and functional MRI both at 3T and at ultra-high field (7T and 10.5T), making the data available to the community. On the other hand, the Harvard/MGH consortium is more focused on the development of new sophisticated data analysis and acquisition techniques. Moreover, in Europe, the "developing human connectome project" (dHCP) (http://wp.doc.ic.ac.uk/dhcp/) led by King's College London, Imperial College London and Oxford University has recently been announced. The target is to produce the first dynamic map of development of the human brain in vivo acquiring MRI data from infants ranging from 20 to 44 weeks post-conceptional age. Therefore, this project tackles the tremendous challenges of scanning infant brain in the womb of the mother. By understanding how different part of the brains are connected to each other it is possible to get new insights about how the brain is shaped and works under normal conditions. This new knowledge will then be used in clinical settings and will allow to spot focal incongruences which might underlie some neurological diseases, such as autism spectrum disorder and Parkinson.

Over the last decade several works have already shown the great importance of dMRI for clinical applications (Dong et al., 2004; Hughes, 2007). The contrast provided by dMRI data is widely used in clinical practice, primarily to diagnose acute ischemic stroke. This helps doctors on a daily basis to improve diagnosis of a suspected stroke patient and guide the need for immediate medication necessary to improve outcome. With the advent of DTI new scalar indices became available about 20 years ago, such as mean diffusivity (MD) and fractional anisotropy (FA). These indices are related to the structural coherence and integrity of white matter fibers and have been increasingly used to map and identify morphological alterations of pathological brain tissue. Such potential can improve diagnosis and treatment planning of 
degenerative diseases such as Alzheimer's disease, Parkinson's disease and Multiple Sclerosis. Furthermore, DTI-based tractography allows tracing white matter connections in vivo, as described in this thesis. When dealing with patients affected by diseases such as brain tumors, neurosurgeons can be provided with a three dimensional map of brain fibers around the affected tissue, making presurgical planning more precise. This means that it is now possible to avoid the loss of cognitive or physical capabilities in a patient as a consequence of a neurosurgeon having blindly removed a 'safe' amount of brain tissue around the tumor to eliminate the health threat.

Unfortunately, this great potential in advancing medical diagnostic MRI brain imaging has found quite some resistance when trying to enter into the hospital routine. This is mainly because dMRIbased techniques are considered to be slow, due to the big amount of data required for every subject and not to be validated enough. In this thesis we have shown that both these points can be easily overcome. From the acquisition point of view, we have tested and proposed several protocols that use multi-band imaging and in plane acceleration techniques. These protocols allow to acquire the data needed for an accurate description of the brain microscopical structure in times which are compatible with those usually available in clinical settings (less than five minutes). This shows that even the most recent dMRI-based techniques, which require the acquisition of hundreds of images for every subject, can be accessible to clinicians. Moreover, we have also shown that data processing can become a matter of few minutes, especially if using the optimal algorithms which we have implemented and used in this work. Using modern acceleration hardware, we have shown that computation times can be greatly reduced. This means that mapping an entire human macroscopic connectome using advanced techniques and GPU acceleration can be done in reasonable time, as shown in chapter 2, providing excellent clinical potential. Furthermore, given the incredible speed which is becoming achievable using such 
methodologies and hardware, it will be possible to provide neurosurgeons with real time visualization of nerve fibers based on the constantly updated position of their surgical instruments. 


\section{References}

Behrens, T.E., Berg, H.J., Jbabdi, S., Rushworth, M.F., Woolrich, M.W., 2007. Probabilistic diffusion tractography with multiple fibre orientations: What can we gain? Neuroimage $34,144-155$.

Cook, P.A., Bai, Y., Nedjati-Gilani, S., Seunarine, K.K., Hall, M.G., Parker, G.J., Alexander, D.C., 2006. Camino: open-source diffusion-MRI reconstruction and processing. 14th Scientific Meeting of the International Society for Magnetic Resonance in Medicine, Seattle, WA, USA.

Dong, Q., Welsh, R.C., Chenevert, T.L., Carlos, R.C., Maly-Sundgren, P., Gomez-Hassan, D.M., Mukherji, S.K., 2004. Clinical applications of diffusion tensor imaging. J Magn Reson Imaging 19, 6-18.

Gerhard, S., Daducci, A., Lemkaddem, A., Meuli, R., Thiran, J.P., Hagmann, P., 2011. The connectome viewer toolkit: an open source framework to manage, analyze, and visualize connectomes. Front Neuroinform 5, 3.

Hughes, J.R., 2007. Autism: the first firm finding = underconnectivity? Epilepsy Behav $11,20-24$

Leemans, A., Jeurissen, B., Sijbers, J., Jones, D.K., 2009. ExploreDTI: a graphical toolbox for processing, analyzing, and visualizing diffusion MR data. 17th Annual Meeting of Intl Soc Mag Reson Med, Hawaii, USA.

Sporns, O., Tononi, G., Kotter, R., 2005. The human connectome: A structural description of the human brain. PLoS Comput Biol 1, e42.

Van Essen, D.C., Ugurbil, K., 2012. The future of the human connectome. Neuroimage $62,1299-1310$. 
Appendix 


\section{Acknowledgements}


Back in 2007, I was approaching the end of my bachelor studies. One day I went to ask a professor at the University of Trieste whether he would be my supervisor for the bachelor thesis. I met his assistant, who told me that there were several choices available and, in case I was interested, there was the possibility to develop one topic abroad. The research topic was dealing with diffusion MRI and the place where I should have gone for three months was Maastricht, in the Netherlands. After the assistant left, I started telling my friend, who was there during the whole conversation, that I did not even know where Maastricht was on the map and that I would have rather preferred to go to the UK. My friend told me that it sounded like a cool opportunity and that I should try it: in the end, it was only for three months. It was a very good suggestion. Three months became very rapidly seven years, and now there are many people I wish to thank for having made this period very special.

Dear Alard, we met already at the time of my bachelor internship and, since then, you have helped and motivated me a lot. During all these years I always had the impression that we were on the same wavelength. Whenever I was stuck, you always had time to discuss things with me and gave me great new ideas. Most of my officemates (and me) will always remember the enthusiastically loud and productive sessions during which we commented some new results while sitting in front of my computer screen.

Dear Rainer, you truly are a real inspirational model for me. In our meetings I have always been impressed by your combination of kindness, knowledge and creativity. After every single meeting, I always felt as if most of the problems were gone, and the ones that were still there were extremely easy.

Dear Jon, thank you for letting me join your group and for sharing your time during the last years. 
Roberta, my life in Maastricht would have definitely not been the same without you. At the beginning it was not easy, though. There is one episode I will always remember (on top of many others): the first time that Anna introduced us to each other. It was winter, you were completely covered by your scarf, hat, etc. and you did not say a single word to me. I only found out several years after, because you actually told me, that I did not look so nice and friendly back then. My being a snobbish northern Italian was playing a strong role there, apparently. Looking back, after all these years, it is something that makes me laugh a lot. You have been the best colleague, housemate and friend I could have possibly asked for. Sharing this part of my life with you has been real fun. Thank you for everything.

Martin, thank you for having made me come through the dangers and the food of some exotic countries. I am still amazed by how you managed to cope with my constant complaints. Luckily for you, the jet lag did not destroy my sleeping capabilities, otherwise it might have been even worse. Joking aside, it has been great having you in Maastricht. I have always enjoyed your sense of humor and admired your cleverness. Whenever I needed to chill a bit or ask for some help, you have always been there. Thank you.

Alexandros, thank you for all the good coffees and discussions about neuroanatomy. I have always found both of them extremely useful and I have always been impressed by your vast knowledge. Thank you for having brought me back to the great world of vinyl records as well. Our trips to Utrecht to look for the best deals in a sea of LPs while Darth Vader and the imperial guards were wandering around have always been great fun.

Joao, you brought a great amount of positive Portuguese energy into Maastricht. I might not be the greatest party animal on earth, but I can say that you made me enjoy most of my nights out in Maastricht and elsewhere. Moreover, I have always been amazed by the enthusiasm and passion that you put in each of your projects. Even! 
Lars, it was great being your housemate and discovering tea varieties which I have never even heard of. It was also great going to play tennis with you, even though you are absolutely unbeatable... as in any other sport that you have ever played in your life, I would say. I have always wondered where all your energy comes from.

Tommaso, from the house on top of the best Maastricht atelier to the last one we shared, we have always had great fun together. Thanks for having immensely broadened my culinary knowledge and skills and for having started me into photography.

Giancarlo, you are probably the smartest and most knowledgeable person I have the pleasure to know. And, during these last years, you have also been a very good friend. Thanks for the time you have shared with me, being it for a nice relaxing chat or helping me with some urgent matters. And thank to you and Salome for the several great lunches and dinners we had together.

Marin, thanks for having been a great officemate and friend. And for having improved my peripheral visual skills when it comes to gratings. It was always great fun with you in the office and chilling at the Coffee Lovers during lazy weekends.

Felix, pal, thank you for all the great conversations and jokes. I enjoyed a lot sharing the office with you and I am very thankful for all your extremely good suggestions and comments about several things that I wrote.

Anna, in my life I would have never thought to share the same working space with a sport star. But, apparently, thanks to you, it happened. I really admire your dedication and strength. I wish to thank you for the nice atmosphere that you contributed to set up in our office.

Aline, thank you for all your nice party vibes. They managed to affect even me and that, I would say, is quite an achievement. 
Britta, thank you for the great company, days and evenings out and very long discussions about how a dot should move on an almostspherical surface.

Kamil, thanks for all the great movies and the nice evenings out. You are the true conference king and that has always impressed me. I once asked you your secret when we were together at the ISMRM in Australia and the answer is something that I will always try to remember.

Silvia, it was really nice having you in Maastricht during the last year. Thank you for having been a great colleague and for the nice dinners we had together.

Vittoria and Julia, thank you for having been my last housemates in Maastricht. It was great sharing the house with you.

I hope I will not forget anyone, but I am very thankful also to all these people that contributed to make my time in Maastricht extremely nice: Elia, Federico, Gojko, Rosanne, Arne, Mario A., Mario S., Mehrdad, Sanne, Arko, Gesa, Judith E., Judith P., Joel, Joost, Dimo, Sven, Jeannette, Martin, Francesco, Alex, Teresa, Thomas, Franziska, Helen, Roy, Valentin, Inge, Sanae, Michelle, Milene, Amanda, Bernadette, Henk, the Bandito crew, Benedikt, Fabrizio, Peter.

Christl and Riny, there are just too many things I should be thanking you for. You are the real operational backbone of the $\mathrm{CN}$ department and you keep it running very efficiently. Thank you for your constant help and kindness.

Lorenzo, dopo quasi oramai 18 anni sei praticamente diventato un fratello per me. Grazie per essermi sempre vicino e per le grandi risate che, nonostante la distanza, continuiamo a farci.

Francesco, se non fosse stato per te, probabilmente a Maastricht non ci sarei mai andato. Grazie per avermi spronato e per continuare a scarrozzarmi in giro (soprattutto, alla Costa dei Barbari). 
Salvatore, grazie per avermi insegnato tutto quello che so sul mondo dell'audio e, anche, in buona parte, su quello della musica. Un capo in b...IN B IN B!

Alessandro, non abbiamo ancora mangiato pasta e fagioli assieme, ma posso dire che, comunque, siamo a un buon livello di conoscenza. Scherzi a parte, grazie per l'ottima copertina di questa tesi e per la tua amicizia.

Francesca, grazie per prenderti cura di tutti noi e per esserci da sempre. La tua cultura vastissima e il tuo umorismo fanno in modo che tutte le conversazioni avute con te siano sempre un piacevole arricchimento. Giulio, grazie per l'olio e per la tua grande bontà d'animo.

Zio Tonino, parte di questa esperienza a Maastricht è stata possibile anche grazie a te. Grazie mille anche per avermi insegnato (e per continuare a farlo) tutto quello che so sulla fotografia.

Infine, il ringraziamento più grande va alla mia famiglia. Grazie a mia madre e mio padre per avermi sempre seguito e supportato con entusiasmo in tutte le mie scelte, sprondandomi a fare sempre ciò che mi piace e seguire le mie passioni. Grazie a Federico, per prendersi cura anche di me. E un grazie speciale a mia sorella Federica, il mio orgoglio e la voce saggia che guida la mia vita. 


\section{Curriculum Vitae}

Matteo Bastiani was born in Trieste, Italy on the $17^{\text {th }}$ of July 1985. After his high school studies he enrolled at the University of Trieste to study biomedical engineering. After he received a three month Socrates scholarship, he joined the department of cognitive neuroscience at the faculty of psychology and neuroscience of Maastricht University, the Netherlands. After graduating, he continued studying engineering and neuroscience concurrently. He graduated both in Italy, where he was enrolled in the master track of clinical engineering at the University of Trieste, and in the Netherlands, where he was selected for the research master in cognitive neuroscience at Maastricht University. He started his $\mathrm{PhD}$ in the framework of the Marie Curie initial training network in October 2010 under the supervision of dr. Alard Roebroeck. 


\section{Publications and talks}

- Peer-reviewed works

*: authors contributed equally to this work

Bastiani, M., Oros-Peusquens, A., Seehaus, A., Brenner, D., Moellenhoff, K., Celik, A., Felder, J., Matusch, A., Bratzke, H., Shah, N.J., Galuske, R., Goebel, R., \& Roebroeck, A. (revision under review). Automatic classification of human cortical layers and areas using high resolution diffusion MRI.

Seehaus, A., Roebroeck, A., Bastiani, M., Fonseca, L., Bratzke, H., Lori, N., Vilanova, A., Goebel, R., \& Galuske, R. (submitted). Histological validation of high-resolution DTI with structure tensor analysis in human post mortem tissue.

Timmers, I., Zhang, H., Bastiani, M., Jansma, B., Roebroeck, A., \& Rubio-Gozalbo, E. (submitted). Revealing white matter microstructure in classic galactosemia using neurite orientation dispersion and density imaging: a case-controlled observational diffusion weighted imaging study.

Graewe, B., Bastiani, M., Duecker, F., Roebroeck, A., Castelo-Branco, M., \& De Weerd, P. (submitted). 3D shading and motion cue combinations: Suppressive competition instead of integration.

Goulas*, A., Bastiani*, M., Bezgin, G., Uylings, H., Roebroeck, A., \& Stiers, P. (2014). Comparative analysis of the macroscale structural connectivity in the macaque and human brain. PLOS Computational Biology, 10(3): e1003529. doi: 10.1371/journal.pcbi.1003529 
Prčkovska, V., Achterberg, H.C., Bastiani, M., Pullens, P., Balmashnova, E., ter Haar Romeny, B.M., Vilanova, A., \& Roebroeck, A. (2013). Optimal Short-Time Acquisition Schemes in High Angular Resolution Diffusion-Weighted Imaging. International Journal of Biomedical Imaging, vol. 2013, Article ID 658583, 17 pages, 2013. doi:10.1155/2013/658583

Bastiani, M., Shah, N.J., Goebel, R., \& Roebroeck, A. (2012). Human cortical connectome reconstruction from diffusion weighted MRI: the effect of tractography algorithm. NeuroImage, 2(3), 1732-49.

- $\quad$ Conference proceedings

Plantinga, B., Bastiani, M., Roebroeck, A., Kuijf, M., Uludag, K., ter Haar Romeny, B., \& Temel, Y. Ultra-high resolution connectivity and parcellation of the human subthalamic nucleus. XXI Congress of the European Society of Stereotactic and Functional Neurosurgery, Maastricht, The Netherlands, September 2014.

Bastiani, M., De Santis, S., Jones, D., Assaf, Y., \& Roebroeck, A. High spatial resolution white matter microstructure with CHARMED and high performance MRI gradients. OHBM Annual Meeting, Hamburg, Germany, 2014.

Bastiani, M., De Santis, S., Jones, D., Assaf, Y., \& Roebroeck, A. Probing white matter microstructure at high spatial resolution combining CHARMED protocol optimization and a high performance gradient set. ISMRM Annual Meeting, Milan, Italy, May 2014.

Fritz, L., Mulders, J., Breman, H., Peters, J., Bastiani, M., Roebroeck, A., Andersson, J., Ashburner, J., Weiskopf, N., \& Goebel, R. Comparison 
of EPI distortion correction methods at 3T and 7T. OHBM Annual Meeting, Hamburg, Germany, 2014.

Harms, R., Bastiani, M., Xu, J., Yacoub, E., Goebel, R., \& Roebroeck, A. White matter microstructure modelling using a modular and extensible gpu accelerated toolkit. OHBM Annual Meeting, Hamburg, Germany, 2014.

Seehaus, A., Roebroeck, A., Fonseca, L., Bastiani, M., Bratzke, H., Lori, N., Vilanova, A., Goebel, R., \& Galuske, R. DTI - its underpinnings on a microstructural basis. OHBM Annual Meeting, Hamburg, Germany, 2014.

Timmers, I., Roebroeck, A., Bastiani, M., Rubio-Gozalbo, E., Jansma, B., \& Zhang, H. Investigating microstructural substrates of white matter changes using NODDI - application to a metabolic disease. ISMRM Annual Meeting, Milan, Italy, May 2014.

Seehaus, A., Roebroeck, A., Fonseca, L., Bastiani, M., Bratzke, H., Lori, N., Vilanova, A., Goebel, R., \& Galuske, R. Structure tensor-based DTI validation in human premotor cortex from 9.4T diffusion MRI. SfN Annual Meeting, San Diego, California, USA, November 2013.

Bastiani, M., Oros-Peusquens, A., Brenner, D., Moellenhoff, K., Seehaus, A., Celik, A., Felder, J., Matusch, A., Galuske, R., Bratzke, H., Shah, N.J., Goebel, R., \& Roebroeck, A. Cortical fiber insertions and automated layer classification in human motor cortex from 9.4T diffusion MRI. ISMRM Annual Meeting, Salt Lake City, Utah, USA, April 2013.

Goulas*, A., Bastiani*, M., Bezgin, G., Roebroeck, A., \& Stiers, P. Comparative quantitative analysis reveals preserved structural connectivity patterns in the human and macaque brain. SFN Annual Meeting, New Orleans, Louisiana, USA, October 2012. 
Bastiani, M., Goebel, R., \& Roebroeck, A. The effect of tractography algorithm on human cortical connectome reconstruction by diffusion weighted MRI. OHBM Annual Meeting, Beijing, China, June 2012.

Bastiani, M., Valente, G., \& Roebroeck, A. Human Connectome Tractography. CONNECT meeting, Tel Aviv, Israel, February 2011.

- Talks

Bastiani, M. Cortical fiber insertions and automated layer classification in human motor cortex from 9.4 Tesla diffusion MRI. ISMRM Benelux Meeting, Rotterdam, January 2013

Bastiani, M. Diffusion-weighted magnetic resonance imaging tractography. Dutch GPGPU day, Amsterdam, The Netherlands, June 2012.

Bastiani, M. The effect of tractography algorithm on human cortical connectome reconstruction by diffusion weighted MRI. ISMRM Annual Meeting, Melbourne, Australia, May 2012.

Bastiani, M. The effect of tractography algorithm on human cortical connectome reconstruction by diffusion weighted MRI. ISMRM Benelux Meeting, Louvain, Belgium, January 2012. 